Florida International University

FIU Digital Commons

6-21-2019

\title{
Multiscale Model of Cerebral Blood Flow Control: Application to Small Vessel Disease and Cortical Spreading Depression
}

Arash Moshkforoush

Florida International University, amosh005@fiu.edu

Follow this and additional works at: https://digitalcommons.fiu.edu/etd

Part of the Bioelectrical and Neuroengineering Commons

\begin{abstract}
Recommended Citation
Moshkforoush, Arash, "Multiscale Model of Cerebral Blood Flow Control: Application to Small Vessel Disease and Cortical Spreading Depression" (2019). FIU Electronic Theses and Dissertations. 4240.

https://digitalcommons.fiu.edu/etd/4240
\end{abstract}

This work is brought to you for free and open access by the University Graduate School at FIU Digital Commons. It has been accepted for inclusion in FIU Electronic Theses and Dissertations by an authorized administrator of FIU Digital Commons. For more information, please contact dcc@fiu.edu. 


\section{FLORIDA INTERNATIONAL UNIVERSITY}

Miami, Florida

\section{MULTISCALE MODEL OF CEREBRAL BLOOD FLOW CONTROL: APPLICATION}

TO SMALL VESSEL DISEASE AND CORTICAL SPREADING DEPRESSION

A dissertation submitted in partial fulfillment of

the requirements for the degree of

DOCTOR OF PHILOSOPHY

in

BIOMEDICAL ENGINEERING

by

Arash Moshkforoush 
To: Dean John L. Volakis

College of Engineering and Computing

This dissertation, written by Arash Moshkforoush, and entitled Multiscale Model of Cerebral Blood Flow Control: Application to Small Vessel Disease and Cortical Spreading Depression, having been approved in respect to style and intellectual content, is referred to you for judgment.

We have read this dissertation and recommend that it be approved.

Cheng-Xian Lin

Wei-Chiang Lin

Sharan Ramaswamy

Jorge Riera Diaz,Co-Major Professor

Nikolaos Tsoukias,Co-Major Professor

Date of Defense: June 21, 2019

The dissertation of Arash Moshkforoush is approved.

Dean John L.Volakis
College of Engineering and Computing

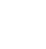

Andrés G. Gil

Vice President for Research and Economic Development and Dean of the University Graduate School

Florida International University, 2019 
(C) Copyright 2019 by Arash Moshkforoush

All rights reserved. 


\section{DEDICATION}

I dedicate this dissertation to my family for their unconditional love and support. 


\section{ACKNOWLEDGMENTS}

I would like to thank my dissertation committee members, colleagues, family, and friends who have extensively helped me during my $\mathrm{PhD}$. Also, I would like to extend my gratitude to the University Graduate School at Florida International University for awarding me the Dissertation Year Fellowship which allowed me to focus on finalizing my dissertation in a timely manner. 


\section{ABSTRACT OF THE DISSERTATION \\ MULTISCALE MODEL OF CEREBRAL BLOOD FLOW CONTROL: APPLICATION \\ TO SMALL VESSEL DISEASE AND CORTICAL SPREADING DEPRESSION}

by

Arash Moshkforoush

Florida International University, 2019

Miami, Florida

Professor Nikolaos Tsoukias, Co-Major Professor

Professor Jorge Riera Diaz, Co-Major Professor

An in-time delivery of oxygen-rich blood into areas of high metabolic demand is pivotal in proper functioning of the brain and neuronal health. This highly precise communication between neuronal activity and cerebral blood flow (CBF) is termed as neurovascular coupling (NVC). NVC is disrupted in major pathological conditions including Alzheimer's disease, dementia, small vessel pathologies (SVD) and cortical spreading depression. Despite the utmost importance of NVC, its underlying mechanisms are not fully understood.

This dissertation presents a multiscale mathematical modeling framework for studying unresolved mechanisms of NVC with major focus on $\mathrm{K}^{+}$ions as a mediator of this process. To this end, models of single-cell electrophysiology are developed for endothelial (EC) and smooth muscle (SMC) cells of capillaries and parenchymal arterioles (PAs). Cells are electrically coupled, and large-scale geometrically-accurate models of microvascular networks are constructed. The change in membrane potential $\left(\mathrm{V}_{\mathrm{m}}\right)$ of PAs in response to 
$\mathrm{K}^{+}$stimulus at different locations of the network are studied under normal and pathological conditions, specifically during SVD and cortical spreading depression. Lastly, the effect of $\mathrm{V}_{\mathrm{m}}$-dependent changes in PA diameter on the regulation of $\mathrm{CBF}$ and hematocrit in the network is analyzed.

Model simulations predict that capillary inward rectifying potassium channels (Kir) enable these cells to sense neuronally-induced changes in extracellular potassium concentrations $\left(\left[\mathrm{K}^{+}\right]_{\mathrm{o}}\right)$ and conduct hyperpolarizing signals over long distances to upstream PAs in an action potential-like regenerative fashion. Simulation results demonstrate that alterations in voltage-gated potassium $(\mathrm{Kv})$ channel density in SVD create a "tug-of-war" dynamic with Kir channels in determining the $\mathrm{V}_{\mathrm{m}}$ and myogenic tone of PA SMCs during NVC. Results also predict a key role of Kir channels in the experimentally observed multiphasic vascular response during high elevations of $\left[\mathrm{K}^{+}\right]_{\mathrm{o}}$ associated with cortical spreading depression. Flow simulations indicate that PA dilation levels and the spatial spread of the hyperpolarizing signal along PAs greatly impact the hemodynamic response in large microvascular networks.

The multiscale models presented in this study were able to accurately capture several experimentally observed responses during NVC and provided insights into their potential underlying mechanisms in health and disease. These models provide a theoretical platform where macroscale, tissue-level responses can be related to microscale, single-cell signaling pathways. These models can help advance the current understanding of mechanisms involved in NVC. 


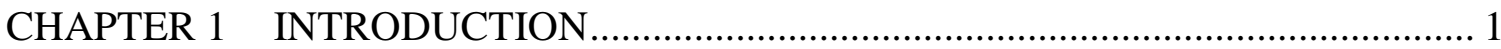

1.1 THE BRAIN AND ITS BLOOD FLOW CONTROL ....................................... 1

1.2 WIDELY ACCEPTED MECHANISMS OF NEUROVASCULAR COUPLING

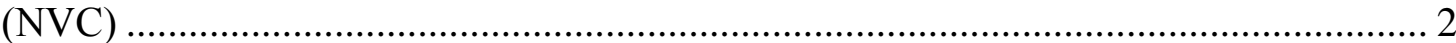

1.3 NEW FINDINGS, CHALLENGES, AND ADDITIONAL MECHANISMS OF

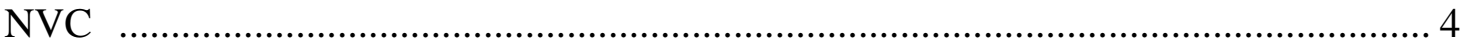

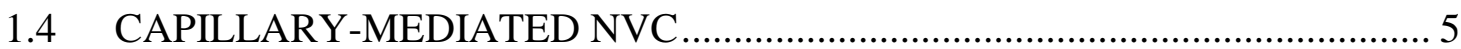

1.5 DISRUPTION OF NVC IN SMALL VESSEL PATHOLOGIES ...................... 6

1.6 NEUROVASCULAR UNCOUPLING IN CORTICAL SPREADING

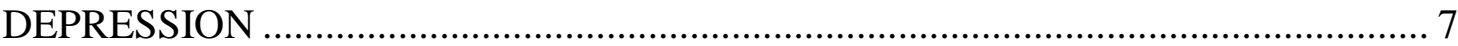

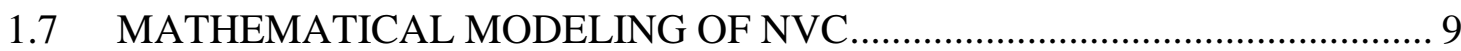

1.8 OVERVIEW OF THIS DISSERTATION …………....................................... 11

\section{CHAPTER 2 COMPUTATIONAL MODELING OF CAPILLARY-MEDIATED}

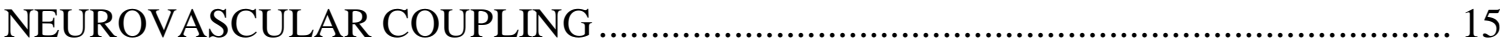

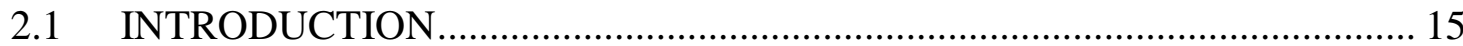

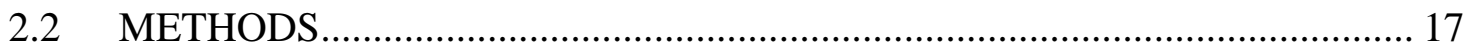

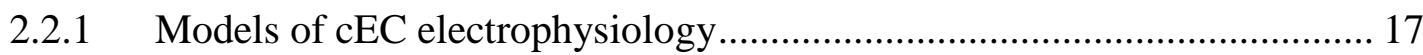

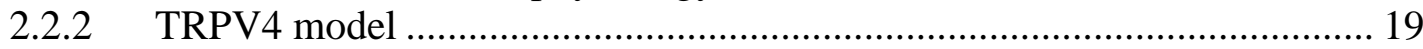

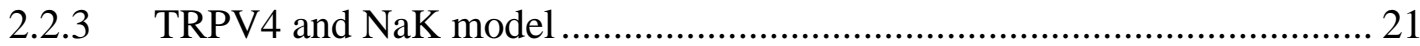

2.3 MODULATION OF TRPV4 AND KIR CURRENTS WITH PIP2 2................ 22

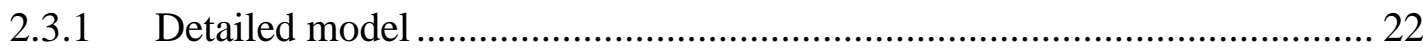

2.3.2 Multicellular models of microvascular networks. ..................................... 23

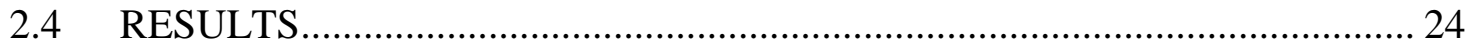

2.4.1 Kir mediates sustained cEC hyperpolarization in

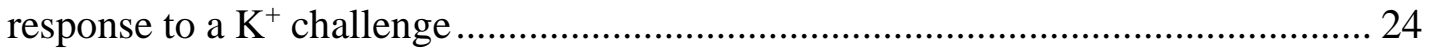

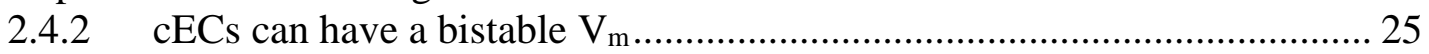

2.4.3 Nak pump and TRPV4 mediate transient $\mathrm{V}_{\mathrm{m}}$ responses during $\mathrm{A} \mathrm{K}^{+}$

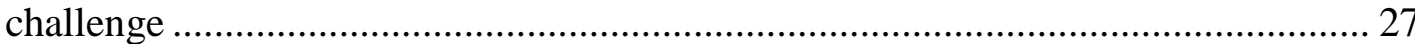

2.4.4 Bistable cells amplify electrical signals and promote hyperpolarizing

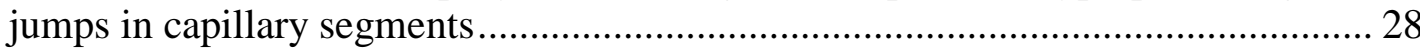

2.4.5 TRPV4 and NaK can facilitate system return following stimulus termination.............................................................................................. 30

2.4.6 Weak intercellular coupling promoting regenerative

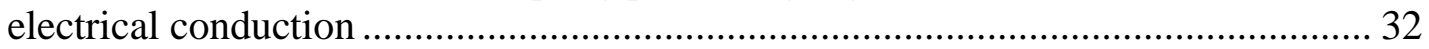

2.4.7 Capillary length, coupling resistance and amplification

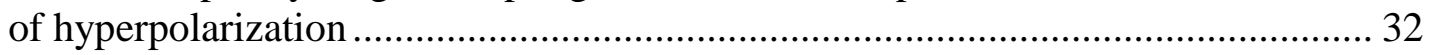

2.4 .8 Conduction velocity ......................................................................... 33

2.4.9 Evidence for regenerative hyperpolarization in the brain microcirculation - an inverted sustained action potential ........................................ 35

2.4.10 Binary vs graded responses of capillary modules to $\mathrm{K}^{+}$stimulus................ 38

2.4.11 Retrograde electrical signaling through capillaries and PAs ...................... 40 


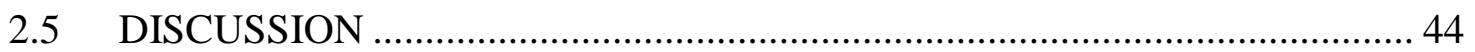

2.5.1 Kir2.1 channel: an exquisite sensor of $\mathrm{K}^{+}$............................................... 44

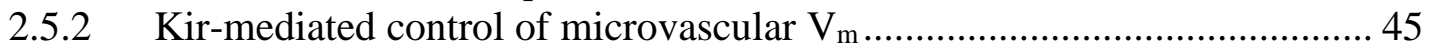

2.5.3 Are ECs capable of carrying APs?............................................................ 46

2.5.4 Evidence for Kir-mediated amplification of electrical signaling................ 46

2.5.5 Analog versus digital mode of microvascular network hyperpolarization . 47

2.5.6 Excitability and AP-like propagation........................................................ 48

2.5.7 PA vs capillary mediated retrograde electrical signaling ............................ 49

CHAPTER 3 DISRUPTION OF NVC IN SMALL VESSEL PATHOLOGIES: A

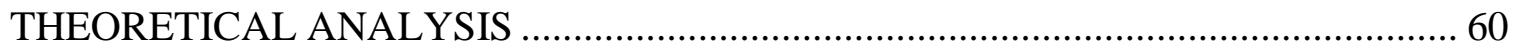

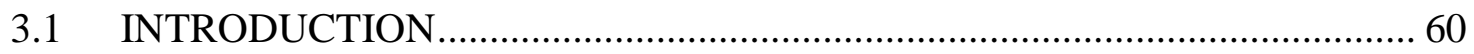

3.2 IMPACT OF KV CHANNEL DENSITY ON THE REGULATION OF THE MYOGENIC TONE IN BRAIN PARENCHYMAL ARTERIOLES...........................63

3.3 IMPACT OF SMALL VESSEL PATHOLOGIES ON THE INTERPLAY

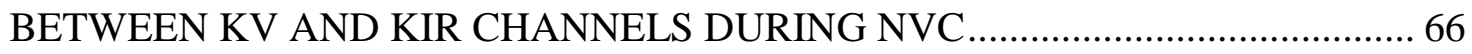

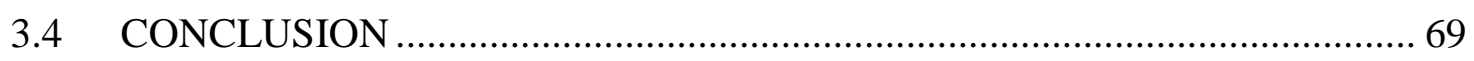

CHAPTER 4 A NEW CURRENT SOURCE DENSITY ANALYSIS METHOD FOR PROPAGATING SOURCES IN THE BRAIN: APPLICATION TO CORTICAL

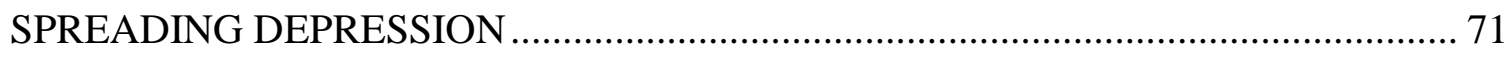

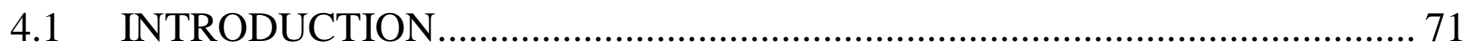

4.2 MATERIALS AND METHODS ……………….......................................... 73

4.2.1 CSD analysis of a planar wave of neocortical neuronal activity

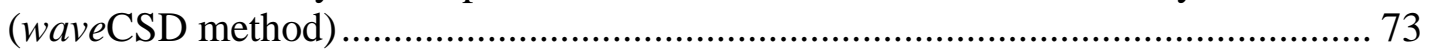

4.2.2 CSD analysis of a propagating wave using iCSD and kCSD methods....... 76

4.2.3 Reconstruction accuracy measures ……………………………………....... 78

4.2.4 Recording LFP data during cortical spreading depression .......................... 79

4.2.5 $\quad c_{\text {waveCSD }}$ associated with cortical spreading depression ............................... 81

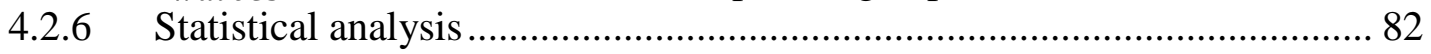

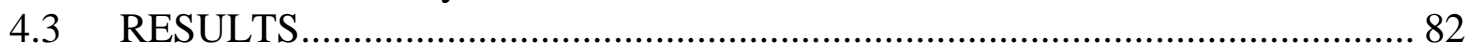

4.3.1 Application of waveCSD, iCSD, and kCSD emthods to neocortical planar waves of neuronal activity …………………………………………...... 82

4.3.2 Robustness of the waveCSD method to noise and MEA resolution........... 89

4.3.3 Errors associated with uncertainties in the knowledge of wave

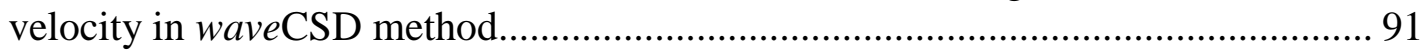

4.3.4 Parameter estimation in waveCSD method using

GCV from the LFP data................................................................................. 93

4.3.5 Transmembrane current sources during cortical spreading depression ...... 94

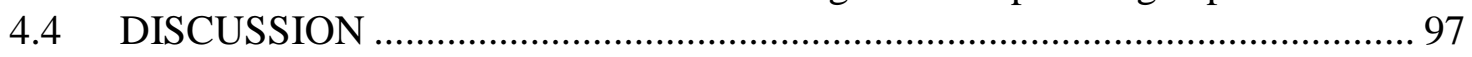

4.4.1 Comparison of waveCSD, kCSD, and iCSD methods in the

CSD analysis of propagating neuronal activity ..................................................... 98

4.4.2 CSD analysis of cortical spreading depression ......................................... 100

4.4.3 Improvement in the waveCSD method and future directions ................... 102

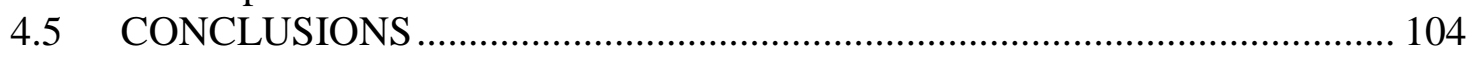




\section{CHAPTER 5 A COMPUTATIONAL ANALYSIS OF THE VASCULAR}

RESPONSE IN CORTICAL SPREADING DEPRESSION ........................116

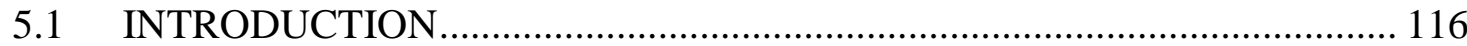

$5.2\left[\mathrm{~K}^{+}\right]_{\mathrm{O}}$ PROFILE DURING CORTICAL SPREADING DEPRESSION ......... 119

5.3 RESPONSE OF BRAIN VASCULATURE TO A WAVE OF HIGH [K $\left.{ }^{+}\right]_{\mathrm{O}} . .121$

5.4 DISCUSSION

\section{CHAPTER 6 HEMODYNAMIC RESPONSE DURING NEUROVASCULAR}

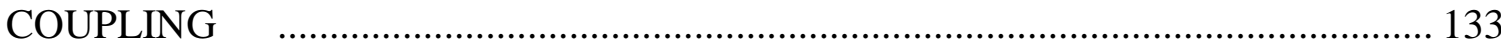

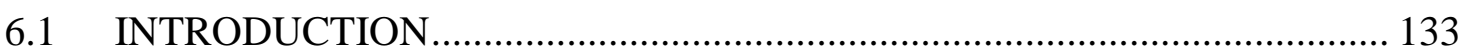

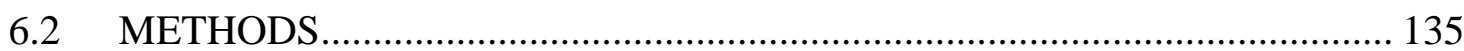

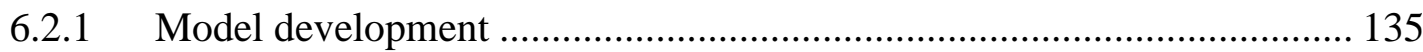

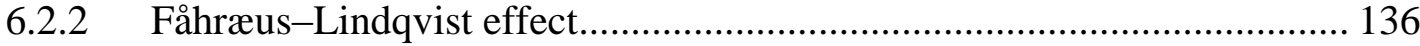

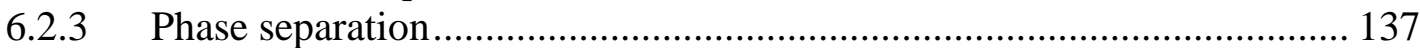

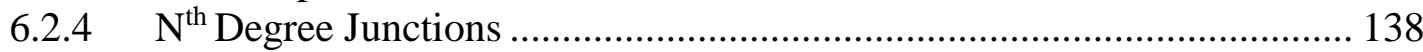

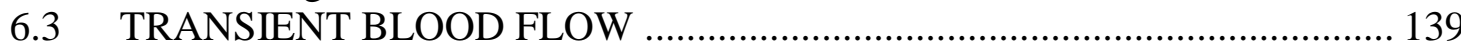

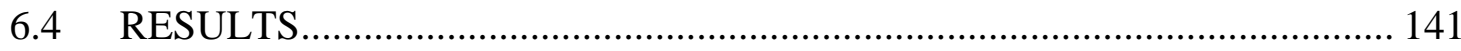

6.4.1 Statistical Properties of the Microvascular Network .............................. 141

6.5 STEADY-STATE BLOOD FLOW AND DISCHARGE HEMATOCRIT .... 142

6.5.1 Effect of Arteriole Dilation and inlet pressure on

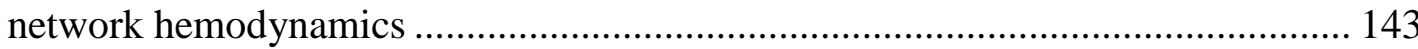

6.6 EFFECT OF THE EXTENT OF UPSTREAM VASODILATION ON

NETWORK HEMODYNAMICS ................................................................. 145

6.7 TRANSIT TIMES OF ERYTHROCYTES IN MICROVASCULAR

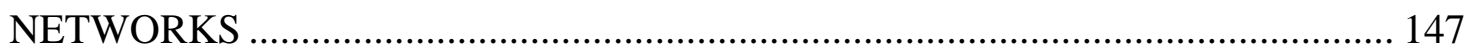

6.8 TRANSIENT BLOOD FLOW IN RESPONSE TO ARTERIOLAR

DILATION............................................................. 148

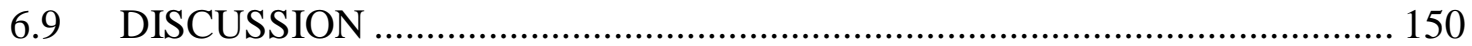

6.9.1 Flow pattern in response to increases in PA diameter and inlet pressure. 151

6.9.2 Distributions of Hematocrit and Plasma due to Phase Separation Laws .. 152

6.9.3 Limitations and future directions ..................................................... 152

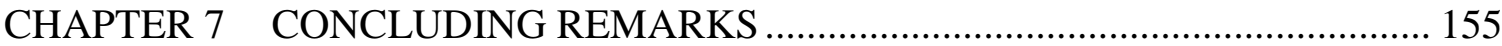

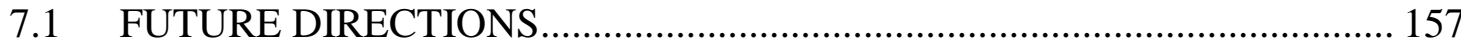

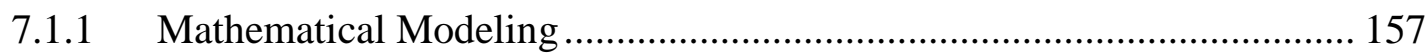

7.1.2 Proposed experimental studies............................................................ 160

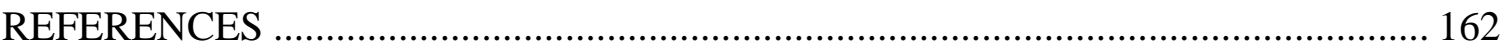

VITA 


\section{LIST OF FIGURES}

FIGURE

PAGE

Figure 2-1 Mathematical models of a single cEC .................................................... 19

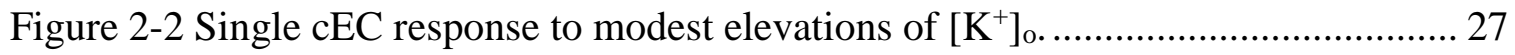

Figure 2-3 Electrical conduction in a capillary segment. ....................................... 31

Figure 2-4 Ex vivo Capillary-PA preparation....................................................... 36

Figure 2-5 Binary and graded hyperpolarization of PAs in vivo................................ 40

Figure 2-6 Parenchymal arteriole vs. Capillary stimulation in vivo.............................. 42

Figure 2-7 Retrograde vasodilatory signaling from capillaries to PAs in vivo. .............. 43

Figure 3-1 KV current density in PA SMCs of normal and diseased animal models at steady state.

Figure 3-2 $V_{m}$ and myogenic tone relation in normal and diseased animal models......... 66

Figure 3-3 Interplay between $\mathrm{Kv}$ with Kir currents in determining $\mathrm{Vm}$ dynamics at rest andduring a potassium challenge.

Figure 4-1 Comparison of the accuracy of waveCSD, iCSD, and kCSD methods in the reconstruction of LFPs generated from a ground-truth waveform.

Figure 4-2 Effect of observation noise on reconstruction accuracy of

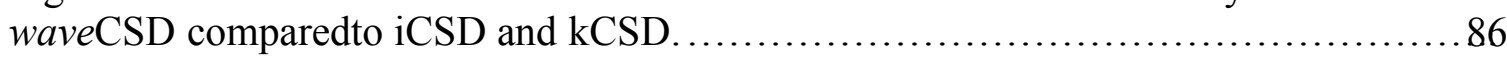

Figure 4-3 Effect of waveform velocity on the reconstruction accuracy of wave CSD, $\mathrm{kCSD}$ and $\mathrm{iCSD}$ methods.

Figure 4-4 Dependency of CSD reconstruction accuracy to the diameter of the designated cylinder used in the iCSD method. 
Figure 4-5 Robustness of the CSD analysis using waveCSD method to noise and electrode resolution.

Figure 4-6 Reconstruction accuracy under uncertainties in the knowledge of velocity of the waveform in the waveCSD method.

Figure 4-7 waveCSD model parameter estimation from the LFP data using GCV technique

Figure 4-8 CSD analysis of LFPs recorded during cortical spreading depression in ratsusing the waveCSD method.

Figure 4-9 Average profile of transmembrane current sources during cortical spreading depression in rats after nonlinear coregistration of the reconstructed profiles

Figure 5-1 Profile of $\mathrm{K}+$ ions in the extracellular space during cortical spreading depression.

Figure 5-2 Response of a segment of endothelial cells to elevations of $[\mathrm{K}+]_{\mathrm{o}}$ during SD.

Figure 5-3 Response of a segment of endothelial cells to elevations of $\left[\mathrm{K}_{+}\right]_{\mathrm{o}}$ during SD.

Figure 5-4 Response of a segment of endothelial cells to a propagating wave of high $[\mathrm{K}+]_{\mathrm{o}}$ during SD.

Figure 5-5 Response of a large network of brain vasculature (reconstructed from [97]) tothe stationary profile of high $[\mathrm{K}+]_{\mathrm{o}}$ during SD.....

Figure 6-1 Statistical features of the microvascular network.

Figure 6-2 Steady state distribution of volumetric blood flow rate (Q) and discharge hematocrit (HD). 
Figure 6-3 Effect of parenchymal arteriole dilation on the steady-state flow distribution in the network.

Figure 6-4 Effect of increasing parenchymal arteriole inlet pressure on the steadystate flow distribution in the network.

Figure 6-5 Effect of the extent of arteriolar dilation on the mean percentage change of blood flow in the network.

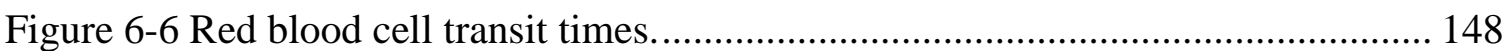

Figure 6-7 Transient simulations of blood flow in the microvascular network.............. 150 


\section{CHAPTER 1 INTRODUCTION}

\subsection{THE BRAIN AND ITS BLOOD FLOW CONTROL}

The human brain comprises of approximately 1000 miles of blood vessels which actively supply nutrients to almost 100 billion neurons [1]. In a healthy brain, the supply of the oxygen-rich blood to areas of high metabolic demand is first initiated from a series of cerebral arteries on the surface of the brain (pial arteries), and then through parenchymal arterioles (PAs) which branch off perpendicularly from these vessels from the VirchowRobin space into the brain parenchyma. PAs deliver blood to a vast network of interconnected capillaries that distribute oxygen and nutrients to active regions of the brain [2].

Proper brain function is hence highly dependent on a rapid and precise response of microcirculation to brain activity and to an efficient distribution of cerebral blood flow (CBF) to active regions over a lifetime. The tightly-regulated mechanism, in which increased neuronal activity is quickly sensed by brain vasculature and is accompanied by a rapid rise in local $\mathrm{CBF}$, is termed as Neurovascular Coupling (NVC) or functional hyperemia. This process, originally discovered in the late nineteenth century by Roy and Sherrington [3], is essential in the prevention of the development of ischemic regions in the brain due to limited energy reserves of neurons and their long-term survival being heavily dependent on blood supply. NVC involves an orchestrated activity of a large group of cells in the brain, including neurons, astrocytes, endothelial cells (ECs), smooth muscle 
cells (SMCs), pericytes, and extracellular components, which are collectively referred to as a neurovascular unit (NVU) [4].

Recent years have witnessed a growing body of research indicating compromised NVC, and the resultant $\mathrm{CBF}$ dysregulation, in major clinical and neurodegenerative conditions including Alzheimer's disease, stroke, hypertension, dementia, and cortical spreading depression, among others [5-9]. The abovementioned neurodegenerative disorders are among the most severe health problems and pose significant economic burden on society. For instance, studies show substantial increase in the prevalence of dementia over the age of $65[10,11]$, with a staggering projected number of $\sim 120$ million people affected by the disease in 2050 [12]. Several studies in literature indicate the strong correlation between cardiovascular risk factors to dementia and cognitive decline [13, 14]. Additionally, above $40 \%$ of vascular dementia cases are attributed to currently untreated Small Vessel Disease (SVD) pathologies $[15,16]$. These findings point to a strong role of microcirculation in neuronal health, and their importance in the prevention of cognitive decline.

\subsection{WIDELY ACCEPTED MECHANISMS OF NEUROVASCULAR COUPLING (NVC)}

Despite the utmost importance of NVC, its exact underlying mechanisms are still debated in the scientific community, even a century after its discovery. Astrocytes, neurons, interneurons, and pericytes have been shown to be involved in NVC [17-23]. The widely accepted mechanism of NVC is that the glutamate release in response to neuronal activity engages metabotropic glutamate receptors (mGluR) in astrocytes and N-methyl-Daspartate receptors (NMDAR) in neurons, initiating cellular pathways leading to the release 
of vasodilatory agents targeting smooth muscle cells (SMCs) of PAs and pial arteries [17]. More specifically, activation of mGluR in astrocytes results in a rise in intracellular calcium concentration $\left[\mathrm{Ca}^{2+}\right]_{\mathrm{i}}$ in the soma through the activation of inositol triphosphate receptors $\left(\mathrm{IP}_{3} \mathrm{Rs}\right)$ on the endoplasmic reticulum (ER). The raised calcium level generates arachidonic acid (AA) from phospholipase A2 (PLA2), which can then convert to prostaglandins (PG) via COX pathway, or epoxyeicosatrienoic acid (EET) by P450 epoxygenase, all of which can relax SMCs of PAs and pial arteries [17].

Additionally, a raised calcium level in the soma initiates a calcium wave that propagates towards astrocytic endfeet, where it activates large-conductance calcium-activated potassium channels $(\mathrm{BKCa})$, and releases potassium ions $\left(\mathrm{K}^{+}\right)$into the perivascular space. The resultant elevation in local $\left[\mathrm{K}^{+}\right]_{0}$, will, in turn, activate inward rectifying potassium channels (Kir) in SMCs of PAs, causing hyperpolarization of these cells. SMC hyperpolarization inactivates voltage-gated calcium channels, which in turn reduces the activity of actin-myosin cross-bridge cycle and leads to the dilation of the vessel and the subsequent increase in CBF [17, 24]. In neurons, activation of NMDA receptors results in the elevation of $\left[\mathrm{Ca}^{2+}\right]$, which can generate nitric oxide (NO) through activation of neuronal nitric oxide synthase (nNOS). NO will generate cyclic guanosine monophosphate (cGMP) to relax SMCs of PAs. The raised calcium levels in neurons might also result in the relaxation of SMCs through the generation of PG via activation of PLA2 and AA [17]. 


\subsection{NEW FINDINGS, CHALLENGES, AND ADDITIONAL MECHANISMS OF NVC}

Studies over the last decade, however, started to cast doubt on the validity of abovementioned mechanisms by questioning the extent of the involvement of astrocytes in the hyperemic response following neuronal activity, as well as the lone contribution of SMCs in NVC [19, 25-28]. In particular, McCaslin and colleagues showed that astrocytes closely encase capillaries and PAs, but they are not detected around pial arteries on the surface of the cortex, limiting their ability to directly influence SMCs of pial arteries [25]. Other studies demonstrated that the calcium rise in astrocytes initiated after the observed diameter increase in the feeding arterioles and pial arteries, raising questions on their involvement in the initiation phase of hyperemic response [26, 28].

Lind and colleagues, however, provided strong evidence that astrocytes in deeper cortical areas, mainly around the capillaries, demonstrated fast $\mathrm{Ca}^{2+}$ responses to a stimulus that preceded observed vasodilations, suggesting the potential involvement of mechanisms initiating vasodilatory signal from deep within the cortex [22]. Chen et al., and Uhlirova et al. also showed that hyperemic response following somatosensory stimulus, is initiated from the brain parenchyma at the capillary level, and is followed by vasodilation in PAs and pial arteries $[19,29]$. Using a light-dye technique (LD) to selectively disrupt the function of endothelial cells, Chen and colleagues demonstrated that stimulus-evoked hyperemic response is significantly halted in post $\mathrm{LD}$ areas in vivo, providing compelling evidence of active involvement of vascular endothelium in NVC [19]. These studies point to the existence of additional mechanisms through which a hyperpolarizing/vasodilatory 
signal is initiated from deeper areas of the cortex and is transmitted rapidly upstream to dilate PAs and surface pial arteries.

\subsection{CAPILLARY-MEDIATED NVC}

Adding to this picture, Longden et al. provided evidence that in addition to SMCs and ECs of PAs, capillary endothelial cells (cECs) isolated from the mouse somatosensory cortex, express functional Kir channels (Kir2.1 subunit) [30]. These channels are a group of seven subfamilies (Kir1-7), which in total have 15 distinct subunits [31]. Kir channels are formed as tetramers of their homo or hetero subunits. This family of potassium channels are called inward rectifiers since their outward current is decreased with the depolarization of the cell membrane positive to the Nernst equilibrium potential of potassium ions $\left(\mathrm{E}_{\mathrm{K}}\right)$, reportedly due to the blockade of the channel pore with intracellular cations such as $\mathrm{Mg}^{2+}$, as well as polyamines $[32,33]$. Increasing the external $\mathrm{K}^{+}$concentration around the Kir channel removes the internal block of cations owing to the increased electrostatic forces and results in the increase in the conductance of the channel [34]. Hence why, Kir channels are termed "ideal sensors" of changes in extracellular $\mathrm{K}^{+}$concentration $\left(\left[\mathrm{K}^{+}\right]\right)$and are reported to have key involvement in NVC [35-40].

Another feature of Kir channels is the presence of a negative slope conductance over the physiological range of membrane potentials $\left(\mathrm{V}_{\mathrm{m}}\right)$ positive to $\mathrm{E}_{\mathrm{K}}$, which results in an increase in channel conductance with hyperpolarization, enabling them to amplify an incoming hyperpolarizing signal [41]. Since neurons are in close proximity to ECs of a highly dense capillary network [42, 43], the abovementioned finding turns cECs into ideal candidates for sensing changes in $\left[\mathrm{K}^{+}\right]_{\mathrm{o}}$ resulting from neuronal activity and, conducting a 
Kir-mediated hyperpolarizing signal upstream to PAs and pial arteries to regulate CBF [44]. Similarly, since $\mathrm{K}^{+}$ions are released in the repolarization phase of each neuronal action potential, which leads to the fluctuations in $\left[\mathrm{K}^{+}\right]_{\mathrm{o}}$ in the extracellular space, the abovementioned finding places $\mathrm{K}^{+}$ions as a one of the most important mediators of NVC.

Both in vivo, as well as in an intact capillary parenchymal arteriole ex vivo preparation, Longden et al. [30] showed significant hyperpolarization and dilation of the PA following modest increases of $\left[\mathrm{K}^{+}\right]_{\mathrm{o}}$ from $\sim 3 \mathrm{mM}$ at resting states to $\sim 10 \mathrm{mM}$ at a distal capillary site. The stimulus-evoked dilation was significantly diminished in EC-specific-knockout (EC Kir2.1 $\left.1^{-/-}\right)$mice or following the application of barium $\left(\mathrm{Ba}^{2+}\right)$, a specific Kir channel blocker. Also, ECKir2.1-/- mice exhibited significantly reduced hyperemic response during whisker stimulation. More recently, the same group under a series of in vivo and ex vivo experiments identified the ATP-dependent plasma membrane phosphatidylinositol 4,5-bisphosphate $\left(\mathrm{PIP}_{2}\right)$ as a key regulator of the activity of Kir2.1 channels in cECs. They observed that physiological PIP2 levels maintain the activity of Kir2.1 channels in cECs. The authors demonstrated that putative NVC mediators, including prostaglandin E2 ( $\left.\mathrm{PGE}_{2}\right)$, can regulate the fidelity of capillary to arteriolar signaling during NVC through activation of Gq-protein-coupled receptor $\left(\mathrm{G}_{\mathrm{q}} \mathrm{PCRs}\right)$ dependent pathways that result in the hydrolysis of $\mathrm{PIP}_{2}$ into diacylglycerol (DAG) and inositol 1,4,5-trisphosphate $\left(\mathrm{IP}_{3}\right)[45,46]$.

\subsection{DISRUPTION OF NVC IN SMALL VESSEL PATHOLOGIES}

Aside from Kir channels, other $\mathrm{K}^{+}$channel subtypes are also present in brain PAs and pial arteries. These channels include voltage-gated $\mathrm{K}^{+}(\mathrm{Kv})$, large conductance $\mathrm{Ca}^{2+}$-activated $\mathrm{K}^{+}(\mathrm{BKCa})$, and ATP-sensitive $\mathrm{K}^{+}$(KATP) channels [47]. Interestingly, in contrast to 
surface pial arteries, in PA SMCs only Kir and Kv channels appear to be active under physiological conditions, as the application KATP agonist cromakalim failed to dilate PAs and the blockade of BKCa channels with paxilline did not change arteriolar diameter in murine animal models [47-50]. Kv channels are a large family of 12 subfamilies (Kv1KV12), with Kv1.2/1.5 being dominant in PAs, which share a common homo- or heteromeric assembly of four pore forming $\alpha$-subunits and auxiliary $\beta$-subunits [51]. These channels play a crucial role in opposing pressure-induced constrictions in vascular SMCs, and consequently determine the vessel tone and along with Kir channels control the diameter of PAs and CBF [47]. The number of functional Kv channels in arteriolar SMCs is altered in small vessel pathologies. Most notably it is significantly increased in cerebral autosomal dominant arteriopathy with subcortical infarcts and leukoencephalopathy (CADASIL) and is substantially reduced in subarachnoid hemorrhage (SAH). These alterations have a profound effect on the interplay between $\mathrm{Kv}$ and Kir channels in determining the $\mathrm{V}_{\mathrm{m}}$ of arteriolar myocytes and subsequently the regulation of $\mathrm{CBF}$ in $\mathrm{K}^{+}$induced NVC [52].

\subsection{NEUROVASCULAR UNCOUPLING IN CORTICAL SPREADING DEPRESSION}

While modest elevations in $\left[\mathrm{K}^{+}\right]_{\mathrm{o}}$ (up to $20 \mathrm{mM}$ ), levels reached during normal $\mathrm{NVC}$, is accompanied by arteriolar SMC hyperpolarization mainly owing to the effect of Kir channels and the sodium-potassium (NaK) pump [47], further increases in $\left[\mathrm{K}^{+}\right]_{\mathrm{o}}$ under pathological conditions such as cortical spreading depression can result in the depolarization of cells and vasoconstriction [53, 54]. First discovered by Leao in mid- 
1940 's, cortical spreading depression is a slow wave $(\sim 3-5 \mathrm{~mm} / \mathrm{min})$ of sustained, nearcomplete neuronal and glial depolarization, lasting approximately for one to two minutes, which silences the synaptic activity in the cortex for an extended period of time (up to 15 minutes) [54-57]. Although primarily linked to migraine aura, cortical spreading depression has also been ascribed to other neurovascular disorders including ischemic stroke, traumatic brain injury and SAH [56, 58]. Cortical spreading depression is characterized by a massive shift of transmembrane ionic and water balances, increased neurotransmitter release, elevation of vasoactive agents such as NO and AA metabolites, and reduced oxygen and glucose levels across the cortex, all of which result in significant alterations in cortical perfusion and the disruption of NVC $[9,54,56,59,60]$. Most notably, $\left[\mathrm{K}^{+}\right]_{\mathrm{o}}$ is reported to reach $\sim 30-60 \mathrm{mM}$, or even in some cases to as high as $\sim 80 \mathrm{mM}[54$, 55]. This massive efflux of $\mathrm{K}^{+}$ions to the extracellular space is countered by the influx of $\mathrm{Na}^{+}, \mathrm{Cl}^{-}$, and $\mathrm{Ca}^{2+}$ ions, resulting in the hypotonicity of the extracellular solution compared to the intracellular space and consequently swelling of cells. Cell swelling, and the subsequent shrinkage of the extracellular space, can result in even further increases in ionic extracellular concentrations [55].

The multitude of vasoactive agents concurrently released during cortical spreading depression, coupled with the high degree of heterogeneity among cell types in different species, makes the understanding and prediction of the vascular response during the cortical spreading depression extremely challenging. Combining optical intrinsic microscopy with electrophysiological recordings, Brennan and colleagues observed a multiphasic response of cortical surface arterioles during cortical spreading depression in 
anesthetized rats, i.e. vessels dilated before and after the wavefront, while they underwent constriction upon the arrival of the wave [53]. Intriguingly, they observed that the vascular response was initiated before the arrival of the wave and propagated with significantly higher velocities than the wavefront itself. Additionally, dilation of the vessels did not follow the path of the wave; rather, it followed the tortuous path of the vessels and it propagated to areas beyond the reach of the cortical spreading depression wavefront. Vascular responses in different species during this phenomenon have also revealed a complex and, although not always the same, in most cases multiphasic patterns [55]. Collectively, these results provide compelling evidence for the existence of distinct conduction of vasodilatory signal along the brain microcirculation, which could in principle have a profound effect on cortical spreading depression-related pathologies, and the neurovascular uncoupling during this phenomenon.

\subsection{MATHEMATICAL MODELING OF NVC}

All examples presented above, point to an unparalleled perplexity of CBF regulation/dysregulation in normally functioning brain and under pathological conditions. The multitude of coexistent cellular pathways, large variability within different animal species, structural complexity and inaccessibility of cerebral microcirculation for experimental studies make mathematical modeling a powerful and essential tool to gain insights into CBF control. Over the past few decades, different aspects of NVC has been the subject of extensive research in the modeling community. There is a great body of literature on mathematical modeling of an NVU with major emphasis on neuronal and glial compartments [61-68]. There are also several studies based on compartmental Balloon and 
Windkessel models on analyzing the hemodynamic response, and the observed blood oxygenation level-dependent (BOLD) signal from functional magnetic resonance imaging (fMRI) data in humans and different animal species [69-79].

Although the abovementioned modeling studies, among many others (which will be discussed more in detail in the following chapters), have significantly advanced our understanding of the underlying mechanisms involved in $\mathrm{NVC}$, majority of the available models rely on the mesoscale compartmental modeling of the phenomenon, in which the combined effect of a large group of cells is modeled as a single compartment. For instance, all SMCs of the PA wall is treated as a single SMC compartment with mesoscale properties. Despite numerous merits associated with compartmental models in NVC, such as the ability to incorporate multiple cell types and making inference on large scale simulations with a low computational expense, these models fail to predict/analyze how localized changes at the microscale (single-cell) level can affect the macroscale (tissue) level responses. Most importantly, recent findings of capillary mediated NVC [30] raises several important questions (e.g. how signals generated at localized areas around the capillaries can hyperpolarize PAs hundreds of microns away without the signal getting dissipated in the vast network of connected cECs, or how capillary level stimulation is compared with PA level stimulation in the conducted responses in vasculature and the resultant $\mathrm{CBF}$ regulation) that requires one to take complicated geometrical features of the brain vasculature and the conduction of electrical signals along interconnected vessels and cell types into account. 


\subsection{OVERVIEW OF THIS DISSERTATION}

The NVC process has primarily been attributed to the direct effect of vasoactive agents, derived from neurons and/or astrocytes, on the arteriolar SMCs [17]. Recent studies, however, have challenged the involvement of astrocytes in this process and the role of SMCs as the sole sensor of neuronal activity in the vasculature [26, 28]. A delayed rise in the astrocytic calcium levels, compared to the dilation of nearby PAs, have been observed, questioning whether astrocytic signals are fast enough to drive the onset of the hyperemic response [26]. On the other hand, calcium mobilization appears to be faster in astrocytes located in deeper regions [22] and retrograde signaling may allow signals initiated deeper in the brain to be transmitted to upstream feeding arterioles and surface arteries [19, 29]. Furthermore, the role of capillaries in NVC has been for the most part ignored. The robust expression of Kir2.1 channels in these cell types may enable capillaries to sense neuronally-induced potassium release into the perivascular space, either directly from neurons or through the activity of astrocytes, and transmit vasodilatory signals to dilate upstream PAs.

The aim of this dissertation is to utilize mathematical modeling along with recent experimental data to investigate the role of capillaries in NVC. The identification of a novel mechanism underlying the hyperemic response will provide a paradigm shift in our understanding of how blood flow is controlled in the brain. A capillary-mediated NVC may enable a more refined regional blood flow regulation and change our perspective on how local is blood flow control in the brain. It can potentially reconcile desperate findings regarding the involvement of astrocytes in NVC, by shifting the focus from the astrocyte- 
to-arteriole to the astrocyte-to-capillary communication. Most importantly, delineating a major signaling mechanism underlying functional hyperemia will improve our understanding of brain function in health and under pathological conditions and provide a theoretical framework for developing targeted therapeutic strategies.

As such, the work presented in this dissertation seeks to provide a multiscale mathematical modeling framework which builds on prior work by our group and others in the field to bridge the gap between our understanding of the microscale signaling and macroscopic tissue-level functional responses in NVC in actual reconstructed brain vascular networks. Our central focus is on pathways involving $\mathrm{K}^{+}$ions in both normally functioning brain as well as in the presence of diseased conditions, including CADASIL, SAH, and cortical spreading depression. To this end, the dissertation is organized as follows:

In CHAPTER 2, through a series of mathematical models with increasing level of detail and complexity, we study capillary mediated NVC with the major focus on the dual role of Kir channels as sensors of neuronal activity and amplifiers of the electrical signal. We conduct our study by modeling a single $\mathrm{cEC}$ response to neuronal activity (through an elevation in $\left[\mathrm{K}^{+}\right]_{\mathrm{o}}$ ) and identify how local changes in the $\mathrm{V}_{\mathrm{m}}$ of these cells can be propagated in a network of gap-connected cECs and PA ECs and SMCs in realistic largescale microvascular networks. We further identify the key determinants of the system which regulate the fidelity and the extent of the conducted responses from cECs to upstream PAs.

In CHAPTER 3, we develop a detailed model of a PA SMC to study how changes in the Kv channel density in SVD, particularly in CADASIL and SAH, affects the arteriolar 
myocyte resting $\mathrm{V}_{\mathrm{m}}$ and the myogenic tone. We also analyze how opposing influences of the NVC-associated modest elevations in $\left[\mathrm{K}^{+}\right]_{\mathrm{o}}$ on $\mathrm{Kv}$ and Kir channels creates a "tug of war" between these channels in determining the resting $V_{m}$ and the response of the cell to a potassium challenge under control and diseased scenarios.

To study the abovementioned multiphasic vascular response during the propagation of the cortical spreading depression wave, in CHAPTER 4, we develop a novel current source density analysis technique, the "waveCSD" method, which is specifically designed to estimate propagating current sources in the cortex, as in the case of cortical spreading depression, from their local field potential (LFP) reflections. We utilize the method to estimate transmembrane current sources, their shape and spatial extent, during the cortical spreading depression from in vivo LFP recordings using microelectrode arrays in rats. The reconstructed wavefront will be used in the subsequent chapter to estimate the spatial profile of released $\mathrm{K}^{+}$ions during this phenomenon.

In CHAPTER 5, we use mathematical models developed in earlier chapters alongside the estimate of the shape and the extent of cortical spreading depression wavefront to predict the underlying cellular mechanisms of the vascular response during extremely high elevations in $\left[\mathrm{K}^{+}\right]_{\mathrm{o}}$ in this phenomenon. To this end, we first study the response of a long series of representative cells, electrically connected through gap junctions, subject to a wave of high $\left[\mathrm{K}^{+}\right]_{\mathrm{o}}$. We take the diffusion of $\mathrm{K}^{+}$ions in the extracellular space as well as the buffering of these ions by astrocytes into account in our simulations. We then perform simulations in large scale microvascular reconstructions, to investigate how massive 
increases in $\left[\mathrm{K}^{+}\right]_{\mathrm{o}}$ can give rise to distinct vascular patterns observed by Brennan and coworkers [53], in a network of interconnected capillaries, PAs, and parenchymal venules.

In CHAPTER 6, using a detailed model of blood flow control, we analyze how vasodilation levels observed in response to arteriolar hyperpolarization in previous chapters can change the transient and steady-state distribution of blood flow and hematocrit in the reconstructed brain microvascular network. In these simulations, we take the interaction between individual red blood cells with the inner walls of rigid capillary vessels, and the noncontinuum nature of blood when flowing through narrow vessels into effect. These simulations provide a platform where cell level electrophysiological simulations can be connected to tissue level blood perfusion. Lastly, in CHAPTER 7, we discuss several potential avenues of further development of the multiscale modeling approach presented and propose experimental procedures for testing the observed model predictions. 


\section{CHAPTER 2 COMPUTATIONAL MODELING OF CAPILLARY-MEDIATED NEUROVASCULAR COUPLING}

The content of this chapter is to be submitted as Moshkforoush A, Ashenagar B, Mirza A, Nelson MT, Tsoukias M. "Computational Modeling of Capillary Mediated Neurovascular Coupling: The Dual Role of Kir Channels as Sensors of Neuronal Activity and Amplifiers of Electrical Signals"

\section{$2.1 \quad$ INTRODUCTION}

Neural activity leads to a rapid increase in local cerebral blood flow by dilating penetrating (parenchymal) arterioles (PAs) and surface (pial) arteries. The process which underlies this functional hyperemia is referred to as neurovascular coupling (NVC) and allows blood supply to match metabolic demands in the brain [80]. NVC is essential for normal brain function and is disrupted in neurodegenerative disorders and stroke [5]. Functional hyperemia also constitutes the physiological basis for functional neuroimaging techniques that are widely used to probe brain function [81].

Despite significant research efforts, how neural activity is sensed by the vasculature is far from being fully understood and the communicating cells and chemical messengers involved are still under debate. It is now recognized that neurovascular coupling mechanisms involve a variety of mediators (including NO, Arachidonic Acid metabolites and $\mathrm{K}^{+}$ions) that are released from neurons, interneurons, or glial cells [17, 18, 36, 82-84]. An emerging paradigm is that astrocytes are the bridges between neurons and the 
vasculature to mediate much of the hyperemic response. Glutamate released by active neurons engages metabotropic glutamate receptors (mGluRs), initiating a $\mathrm{Ca}^{2+}$ signal that reaches the astrocytic endfeet and releases vasoactive substances, including $\mathrm{K}^{+}$, into the perivascular space [17]. An increase in extracellular potassium concentration $\left(\left[\mathrm{K}^{+}\right]_{\mathrm{o}}\right)$, can activate vascular inward rectifying potassium (Kir) channels resulting in vessel hyperpolarization, dilation, and the subsequent increase in cerebral blood flow (CBF) [17, 24].

The arteriolar smooth muscle has been considered the primary target of NVC mediators. Recent studies, however, put the endothelial layer at center stage in NVC. Data suggest that retrograde vasodilatory signals, initiated from deep within the cortex, ascend through the endothelial layer to dilate surface arteries [19, 22, 25-28, 44, 85]. Moreover, evidence of coupling between neuronal activity and the vasculature at the capillary level has been presented [86]. We have recently proposed that brain capillaries act as a neuronal activitysensing network and initiate electrical (hyperpolarizing) signals that ascend to dilate upstream arterioles and increase CBF [30]. In support of this proposition, we have shown that local $\mathrm{K}^{+}$release at distal capillary sites evokes significant Kir2.1-dependent hyperpolarization and dilation of the feeding PA, and that hyperemic responses are significantly compromised in EC-specific Kir2.1 knockout mice (EC Kir2.1 $1^{-/-}$). Thus, data suggest extracellular $\mathrm{K}^{+}$as a critical mediator for capillary-level NVC and the Kir2.1 channel as the key molecular player for sensing neuronal activity-dependent elevations in $\left[\mathrm{K}^{+}\right]_{\mathrm{o}}$ and translating them into retrograde hyperpolarizing signals. 
This study utilizes a mathematical framework to investigate the underlying mechanisms that enable capillaries to sense neuronal-induced changes in $\left[\mathrm{K}^{+}\right]_{\mathrm{o}}$ and transmit vasodilatory signals to upstream PAs. We first examine the membrane potential $\left(\mathrm{V}_{\mathrm{m}}\right)$ dynamics of single capillary endothelial cells (cECs) and analyze the interplay of Kir with the transient receptor potential vanilloid 4 (TRPV4) channel and the $\mathrm{Na}^{+}-\mathrm{K}^{+}$-ATPase pump (NaK). We then form capillary segments by coupling cECs in series via incorporations of gap junctions and examine the biophysical determinants of conducted hyperpolarization in capillaries. Lastly, we examine under what conditions capillary initiated signals can reach upstream feeding arterioles in simulated microvascular networks and investigate mechanisms and parameters that regulate the fidelity of the response.

\subsection{METHODS}

We construct a series of mathematical models to investigate electrical signaling in the brain microcirculation. Patch clamp studies in freshly isolated mouse cerebral cECs revealed the presence of functional Kir2.1 and TRPV4 in these cells, but in contrast to ECs from all other vascular beds examined up to this date, the absence of small (SK) and intermediate (IK) conductance calcium-activated $\mathrm{K}^{+}$channels [85]. We, thus, examine the role of these channels in capillary electrophysiology by constructing a series of models with increasing detail and complexity.

\subsubsection{Models of cEC electrophysiology}

A minimal model of cEC electrophysiology (Model A, Figure 2-1) entails an explicit mathematical description for the Kir current ( $\left.\mathrm{I}_{\text {Kir }}\right)$, while all other transmembrane currents 
are lumped into a non-specific linear background current $\left(\mathrm{I}_{\mathrm{bg}}\right)$ with a lumped conductance, $\mathrm{G}_{\mathrm{bg}}$ :

$$
\begin{aligned}
& I_{\text {Kir }}=\frac{\bar{G}_{\text {Kir }}\left[\mathrm{K}^{+}\right]_{0}^{0.5}\left(V_{m}-E_{K}\right)}{1+\exp \left(\frac{\left(V_{m}-V_{0.5}\right)}{k}\right)} \\
& I_{b g}=G_{b g}\left(V_{m}-E_{b g}\right)
\end{aligned}
$$

where $\mathrm{E}_{\mathrm{bg}}$ is the reversal potential for $\mathrm{I}_{\mathrm{bg}}$; $\mathrm{E}_{\mathrm{K}}$ is the Nernst potential for $\mathrm{K}^{+}$ions; and $\mathrm{V}_{0.5}$ and $\mathrm{k}$ are parameters that describe a sigmoidal inhibition of Kir with membrane depolarization (i.e. $\mathrm{V}_{\mathrm{m}}$ for half maximal inactivation and the steepness factor, respectively). The Boltzmann-type formula (Equation 2-1) captures the IKir's inward rectification and the negative slope conductance as $\mathrm{V}_{\mathrm{m}}$ approaches more depolarized potentials. (i.e. the inhibition of $\mathrm{I}_{\mathrm{Kir}}$ as $\mathrm{V}_{\mathrm{m}}$ depolarizes yields the characteristic $\mathrm{N}$-shaped relationship depicted in Figure 2-2B). The channel's conductance increases with $\left[\mathrm{K}^{+}\right]_{\mathrm{o}}\left(\mathrm{G}_{\mathrm{Kir}}=\overline{\mathrm{G}}_{\mathrm{Kir}} \sqrt{\left[\mathrm{K}^{+}\right]_{\mathrm{o}}}\right)[87$, 88]. The assumed ohmic behavior of $\mathrm{I}_{\mathrm{bg}}$ is validated against a detailed EC electrophysiology model (Figure 2-1D and Appendix A2). Using a standard HodgkinHuxley type formalism, the time-dependent changes in $\mathrm{V}_{\mathrm{m}}$ are predicted from Equation 2-3:

$$
\mathrm{C}_{\mathrm{m}} \frac{\mathrm{dV} \mathrm{m}}{\mathrm{dt}}+\mathrm{I}_{\mathrm{bg}}+\mathrm{I}_{\text {Kir }}=0
$$

where $\mathrm{C}_{\mathrm{m}}$ is the membrane capacitance. $\mathrm{C}_{\mathrm{m}}, \overline{\mathrm{G}}_{\mathrm{Kir}}$, and $\mathrm{G}_{\mathrm{bg}}$ were determined from patch data in freshly isolated mouse cerebral cECs, and $\mathrm{E}_{\mathrm{bg}}$ is approximated as the resting $\mathrm{V}_{\mathrm{m}}(\sim-$ $30 \mathrm{mV}$ ). Kir current-voltage data have been satisfactorily fitted with a k value of $\sim 7 \mathrm{mV}$; however, there is a high degree of ambiguity in literature regarding the value of $\mathrm{V}_{0.5}$, with 
data suggesting that it drifts with $\mathrm{E}_{\mathrm{K}}$ and may vary between $\mathrm{E}_{\mathrm{K}}$ and $\mathrm{E}_{\mathrm{K}}+40 \mathrm{mV}[36,89,90]$.

In all simulations performed in this study, we assume a control value of $\left(E_{K}+25 m V\right)$ for

$\mathrm{V}_{0.5}$, and we examine the effect of varying $\mathrm{V}_{0.5}$ in Appendix B2.
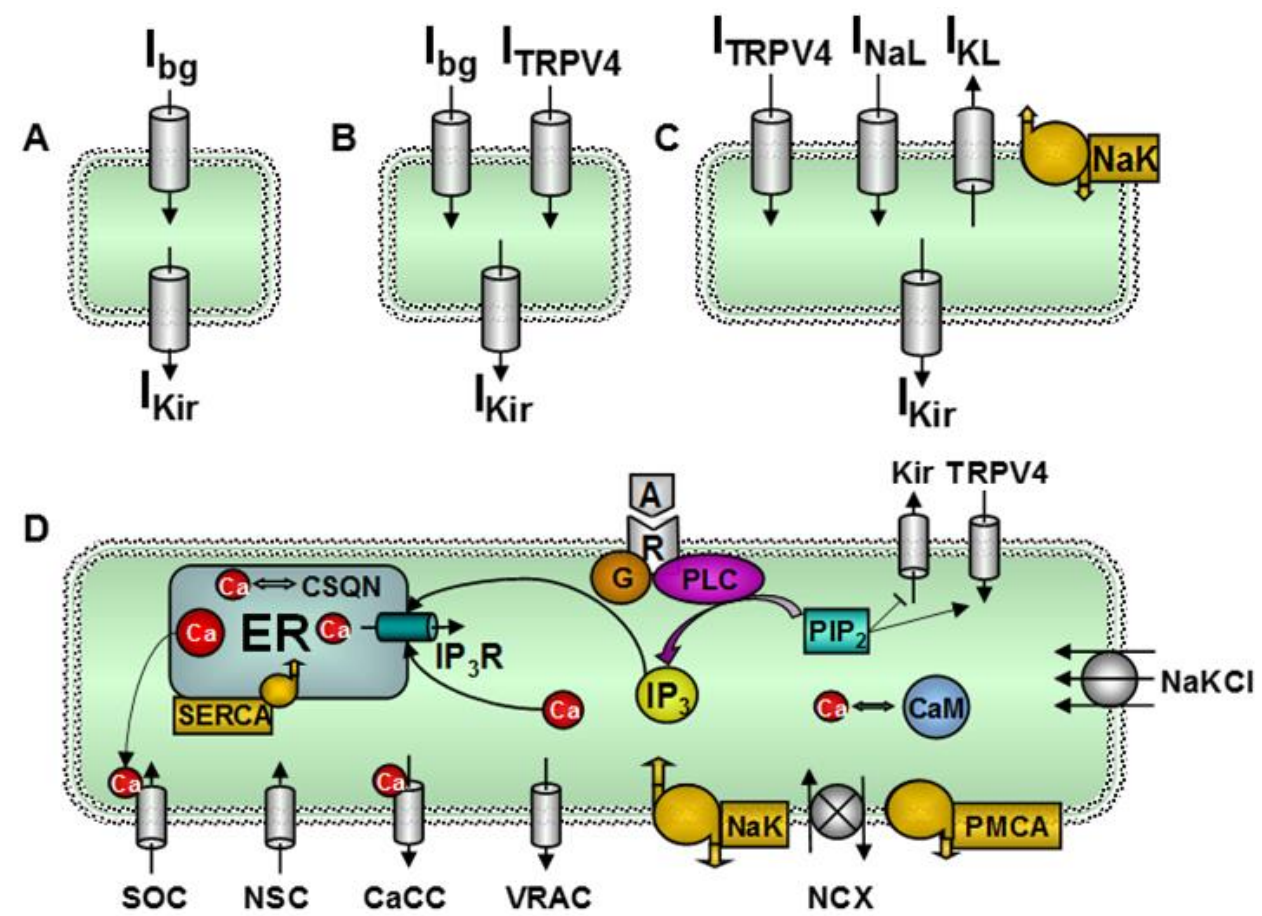

Figure 2-1 Mathematical models of a single cEC. (A) Minimal model of cEC (Model A) includes a description for Kir $\left(\mathrm{I}_{\mathrm{Kir}}\right)$ while the rest of transmembrane currents are lumped into a nonspecific background current $\left(\mathrm{I}_{\mathrm{bg}}\right)$. (B) Model B examines the effect of stochastic opening of TRPV4 on $\mathrm{V}_{\mathrm{m}}$ dynamics by separating the TRPV4 current ( $\mathrm{I}_{\mathrm{TRPV}}$ ) from the lumped background current in model A. C) Model C examines the combined contribution of the NaK pump and TRPV4 currents in the response of a cEC to stimulus. Descriptions for transmembrane $\mathrm{Na}^{+}\left(\mathrm{I}_{\mathrm{NaL}}\right)$ and $\mathrm{K}^{+}\left(\mathrm{I}_{\mathrm{KL}}\right)$ currents close the ionic mass balances and allow intracellular concentrations to drift as the NaK activity changes. D) Detailed cEC model (Model D) modified from Silva et al. [89]. The model incorporates $\mathrm{I}_{\mathrm{Kir}}, \mathrm{I}_{\mathrm{TRPV}}$, and $\mathrm{NaK}$ as in the minimal models and includes currents through non-selective cation (NSC) and Chloride $(\mathrm{Cl})$ channels, PMCA pumps, the NCX exchanger and the $\mathrm{NaKCl}$ cotransport. The model also takes into account the dynamics of intracellular ionic species, second messenger, and major intracellular components.

\subsubsection{TRPV4 model}

To examine the contribution of TRPV4 channels to cEC dynamics, we separated the TRPV4 current (ITRPV4) from the lumped background current in model A (Figure 2-1B).

Model B accounts for the stochastic opening of TRPV4 channels and the resultant transient depolarizing current with bursting and cooperativity kinetics as characterized in mouse 
cECs [46]. We simulated the TRPV4 current using a three-state discrete-time Markov Chain model $[91,92]$. The TRPV4 channel can reside in any of the following three states: I) blocked (intraburst short closed state), II) open, and III) shut (interburst long-closed state). At rest, whole-cell TRPV4 currents from cECs reveal infrequent single level channel openings with an open probability (time-averaged number of open channels $\mathrm{NP}_{\mathrm{o}}=0.038$ ) [46]. In agreement with the experimental data, Model B accounts for one active TRPV4 with bursts of stochastic openings with a mean open time $(90 \mathrm{~ms})$, a mean blocked time (33 ms), a mean shut time (4 min), and a mean burst duration (20 s) [46, 92, 93]. The $\mathrm{Na}^{+}$, $\mathrm{K}^{+}$and $\mathrm{Ca}^{2+}$ currents through an open TRPV4 channel are modeled via a GoldmanHodgkin-Katz equation with the general form:

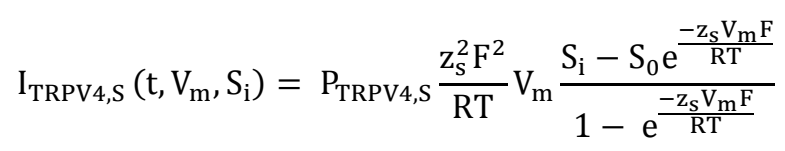

where $\mathrm{I}_{\mathrm{TRPV} 4, \mathrm{~S}}$ is the current carried by each cation $\mathrm{S}$ (i.e. $\mathrm{Ca}^{2+}, \mathrm{K}^{+}, \mathrm{Na}^{+}$); $\mathrm{S}_{\mathrm{i}}$ is the intracellular and $S_{o}$ the extracellular concentration of $S ; z_{S}$ is the valence of the ion; $F$ is the Faradays constant, and $\mathrm{P}_{\mathrm{TRPV} 4, \mathrm{~S}}$ is the ionic permeability $\left(\mathrm{P}_{\mathrm{TRPV} 4, \mathrm{Ca}}=2.5 \times 10^{-8} \mathrm{~cm} / \mathrm{s}\right.$ and $\left.\mathrm{P}_{\mathrm{TRPV} 4, \mathrm{Ca}}: \mathrm{P}_{\mathrm{TRPV} 4, \mathrm{~K}}: \mathrm{P}_{\mathrm{TRPV} 4, \mathrm{Na}}=7.1: 1.4: 1\right)[92]$. The time-dependent net current $\left(\mathrm{I}_{\mathrm{TRPV} 4}(\mathrm{t})\right)$ is the sum of the currents carried by each ion through an open TRPV4 channel, multiplied by the number of open channels at each time, $\mathrm{NP}_{0}(\mathrm{t})$.

$$
\mathrm{I}_{\mathrm{TRPV} 4}(\mathrm{t})=\mathrm{NP}_{0}(\mathrm{t}) \sum_{\mathrm{S}} \mathrm{I}_{\mathrm{TRPV} 4, \mathrm{~S}} \mathrm{P}_{\mathrm{TRPV} 4, \mathrm{~S}}
$$

The dynamics of $\mathrm{cEC} \mathrm{V}_{\mathrm{m}}$ in Model B can therefore be predicted via Equation 2-3, using the following Itot: 


\subsubsection{TRPV4 and NaK model}

Model $\mathrm{C}$ incorporates a description for the activity of the $\mathrm{NaK}$ pump and its dependence on $\left[\mathrm{K}^{+}\right]_{\mathrm{o}}$ and intracellular sodium $\left(\left[\mathrm{Na}^{+}\right]_{\mathrm{i}}\right)$. In this model, the lumped background current in model A is divided into currents carried by TRPV4, a low-affinity isoform of sodium potassium pump (NaK), and generic $\mathrm{Na}^{+}$and $\mathrm{K}^{+}$leak currents (Figure 2-1C). The leak currents are introduced to ensure ionic balance at physiological levels and that "background" currents in models $\mathrm{C}$ and A are equivalent. They also allow intracellular ionic concentrations to drift during sustained stimulations. Here, the dynamics of $\mathrm{NaK}$ pump current is modeled using two sigmoidal relationships sensitive to changes in intracellular $\mathrm{Na}^{+}$and extracellular $\mathrm{K}^{+}$concentrations, respectively (Equation 2-7).

$$
\mathrm{I}_{\mathrm{NaK}}=\frac{\mathrm{I}_{\mathrm{NaK}, \max }}{\left(1+\mathrm{e}^{\left(\left(\mathrm{K}_{\mathrm{K}_{\mathrm{o}}}-\mathrm{K}_{\mathrm{o}}\right) / \mathrm{m}_{\mathrm{K}_{\mathrm{o}}}\right)}\right)\left(1+\mathrm{e}^{\left(\left(\mathrm{K}_{\mathrm{Na}_{\mathrm{i}}}-\mathrm{Na}_{\mathrm{i}}\right) / \mathrm{m}_{\mathrm{Na}_{\mathrm{i}}}\right)}\right)}
$$

where $\mathrm{I}_{\mathrm{NaK} \text {,max }}$ is the maximum $\mathrm{NaK}$ current; $\mathrm{K}_{\mathrm{K}_{0}}$ and $\mathrm{K}_{\mathrm{Na}_{\mathrm{i}}}$ are half maximal activations of $\mathrm{NaK}$ pump to extracellular $\mathrm{K}^{+}$and intracellular $\mathrm{Na}^{+}$, respectively; and $\mathrm{m}_{\mathrm{K}_{\mathrm{o}}}$ and $\mathrm{m}_{\mathrm{Na}_{\mathrm{i}}}$ are sodium and potassium slope factors. Leak currents are modeled as ohmic currents with leak conductances for $\mathrm{Na}^{+}$, i.e. $\mathrm{G}_{\mathrm{Na}}$, and $\mathrm{K}^{+}$ions, i.e. $\mathrm{G}_{\mathrm{K}}$.

$$
\begin{array}{lr}
\mathrm{I}_{\mathrm{NaL}}=\mathrm{G}_{\mathrm{Na}}\left(\mathrm{V}_{\mathrm{m}}-\mathrm{E}_{\mathrm{Na}}\right) & \text { Equation 2-8 } \\
\mathrm{I}_{\mathrm{KL}}=\mathrm{G}_{\mathrm{K}}\left(\mathrm{V}_{\mathrm{m}}-\mathrm{E}_{\mathrm{K}}\right) & \text { Equation 2-9 }
\end{array}
$$

where $\mathrm{E}_{\mathrm{Na}}$ is the reversal potential for $\mathrm{Na}^{+}$ions. The ionic and membrane potential dynamics of model $\mathrm{C}$ can be summarized using following system of equations: 


$$
\begin{aligned}
& \frac{\mathrm{dK}_{\mathrm{i}}}{\mathrm{dt}}=\frac{-\mathrm{I}_{\mathrm{KL}}+2 \mathrm{I}_{\mathrm{NaK}}-\mathrm{I}_{\mathrm{Kir}}-\mathrm{I}_{\mathrm{TRPV} 4, \mathrm{~K}}}{\mathrm{z}_{\mathrm{K}} \mathrm{Fvol}} \\
& \frac{\mathrm{dNa}}{\mathrm{dt}}=\frac{-\mathrm{I}_{\mathrm{NaL}}-3 \mathrm{I}_{\mathrm{NaK}}-\mathrm{I}_{\mathrm{TRPV} 4, \mathrm{Na}}}{\mathrm{z}_{\mathrm{Na}} \mathrm{FVol}} \\
& \mathrm{C}_{\mathrm{m}} \frac{\mathrm{dV_{ \textrm {m } }}}{\mathrm{dt}}=-\mathrm{I}_{\text {tot }} \\
& \mathrm{I}_{\text {tot }}=\mathrm{I}_{\mathrm{NaL}}+\mathrm{I}_{\mathrm{KL}}+\mathrm{I}_{\mathrm{Kir}}+\mathrm{I}_{\mathrm{NaK}}+\mathrm{I}_{\mathrm{TRPV} 4}
\end{aligned}
$$

Equation 2-12

Equation 2-13

\subsection{MODULATION OF TRPV4 AND KIR CURRENTS WITH PIP2}

It has recently been reported that putative NVC mediators, including prostaglandin E2 ( $\left.\mathrm{PGE}_{2}\right)$, activate Gq-protein-coupled receptor $\left(\mathrm{G}_{\mathrm{q}} \mathrm{PCRs}\right)$ dependent pathways that result in the hydrolysis of plasma membrane phosphatidylinositol 4,5-bisphosphate $\left(\mathrm{PIP}_{2}\right)$ into diacylglycerol (DAG) and inositol 1,4,5-trisphosphate ( $\left.\mathrm{IP}_{3}\right)$. Harraz et al. [45, 46] demonstrated that depletion of PIP2 gradually inhibits the Kir channels, and conversely, increases the activity of TRPV4 channels with time constants of $\sim 5-7$ minutes. In some simulations performed in this study, we have incorporated the effect of the dynamic regulation of Kir and TRPV4 through an exponential decrease in $\mathrm{I}_{\mathrm{Kir}}$, and an exponential increase in $\mathrm{I}_{\mathrm{TRPV}}$, with their respective time constants upon start of the potassium stimulus.

\subsubsection{Detailed model}

A detailed model of endothelial membrane electrophysiology, $\mathrm{Ca}^{2+}$ and ionic dynamics [89] was adapted to describe cerebral cEC dynamics (Model D). The model incorporates Kir and TRPV4 currents as described above and assumes absence of IK and SK. The Ibg is partitioned to currents through non-selective cation (NSC) and Chloride (Cl) channels, $\mathrm{NaK}$ and plasma membrane $\mathrm{Ca}^{2+}$-ATPase (PMCA) pumps, the $\mathrm{Na}^{+} / \mathrm{Ca}^{2+}$ exchanger (NCX) 
and the $\mathrm{Na}^{+}-\mathrm{K}^{+}-\mathrm{Cl}^{-}$cotransport $(\mathrm{NaKCl})$. Equations and parameters for these currents are described in [89] and are proportionally scaled here to provide an overall conductance at rest equal to $\mathrm{G}_{\mathrm{bg}}$. Thus, the contribution of $\mathrm{I}_{\mathrm{Kir}}$ and $\mathrm{I}_{\mathrm{TRPV}}$ relative to all other transmembrane "background" currents is maintained in the detailed model (Appendix A2), consistent with the minimal models and experimental data. Furthermore, the detailed model can predict dynamic changes in intracellular ionic concentration, account for the role of pumps/exchanger and test simplifying assumptions in the minimal models.

\subsubsection{Multicellular models of microvascular networks}

cECs were placed in series and coupled through gap junctions to construct multicellular capillary networks, in silico. Current flow between adjacent cECs is estimated based on the electrical gradient and the cEC-to-cEC coupling resistance $\left(\mathrm{R}_{\mathrm{gj}}\right)$.

$$
I_{g, i}=\sum_{n} \frac{1}{R_{g j, i, n}}\left(V_{m, i}-V_{m, n}\right)
$$

Equation 2-14

where $\mathrm{n}$ is the index of cells connected to cell(i), $\mathrm{I}_{\mathrm{gj}, \mathrm{i}}$ is the gap junctional current flowing from cell(i) to cell(n) with a gap junctional resistance $\mathrm{R}_{\mathrm{gj}, \mathrm{I}, \mathrm{n}}$. $\mathrm{R}_{\mathrm{gj}}$ has not been measured in capillaries, we thus examine $\mathrm{R}_{\mathrm{gj}}$ values ranging from $1 \mathrm{M} \Omega$ to $1 \mathrm{G} \Omega$ and assume a control value of 10 or $50 \mathrm{M} \Omega$ based on estimates for coupling resistances between ECs in arteries $[94,95]$. Capillary networks were constructed with a geometry that approximates isolated ex-vivo preparations [30] or were adapted from larger reconstructed microvascular networks [96]. Conduction of electrical signals in PAs was accounted by considering PA EC and SMC membrane resistivities $\left(\mathrm{R}_{\mathrm{m}, \mathrm{EC}} \mathrm{R}_{\mathrm{m}, \mathrm{SMC}}\right)$ and $\mathrm{Kir}$ conductances (G $\mathrm{G}_{\mathrm{Kir}, \mathrm{EC}}$ $\left.\mathrm{G}_{\mathrm{Kir}, \mathrm{SMC}}\right)$ and the myoendothelial gap junctional resistance $\left(\mathrm{R}_{\mathrm{ME}}\right)$ as previously described 
$[94,97]$. Three ECs are coupled to three SMCs at each longitudinal position and for each EC-SMC pair, we account for a total Kir conductance $\left(\mathrm{G}_{\mathrm{Kir}, \mathrm{EC}-\mathrm{SMC}}=\mathrm{G}_{\mathrm{Kir}, \mathrm{EC}}+\mathrm{G}_{\mathrm{Kir}}, \mathrm{SMC}\right)$ and a background conductance $\mathrm{G}_{\mathrm{bg} \text {,EC-SMC }}$ as the inverse of the resting transmembrane resistance, $R_{m, E C-S M C}$. The latter is estimated from the parallel connection of $R_{m, E C}$ with $R_{M E}$ and $\mathrm{R}_{\mathrm{m}, \mathrm{SMC}}$ combined in series:

$$
\mathrm{R}_{\mathrm{m}, \mathrm{EC}-\mathrm{SMC}}=\frac{\left(\mathrm{R}_{\mathrm{m}, \mathrm{SMC}}+\mathrm{R}_{\mathrm{ME}}\right) \mathrm{R}_{\mathrm{m}, \mathrm{EC}}}{\left(\mathrm{R}_{\mathrm{m}, \mathrm{SMC}}+\mathrm{R}_{\mathrm{ME}}+\mathrm{R}_{\mathrm{m}, \mathrm{EC}}\right)}
$$

Each unit is coupled to adjacent EC-SMC units with a resistance, $\mathrm{R}_{\mathrm{gj}, \mathrm{PA}}$. Parenchymal venules (PVs) are also treated same as PAs, with the difference that we assume these vessels lack Kir channels. All simulations have been performed in MATLAB 2018b using ode15s, suitalble for solving stiff initial value ordinary differential equations. Parameter values along with their definitions are provided in Table 2-1.

\subsection{RESULTS}

\subsubsection{Kir mediates sustained cEC hyperpolarization in response to a $\mathrm{K}^{+}$challenge}

We use a mathematical modeling approach to investigate the ability of cECs to sense an increase in $\left[\mathrm{K}^{+}\right]_{\mathrm{o}}$. A minimal model of an isolated cEC (Model A; Figure 2-1A) captures the Kir dynamics during a $\mathrm{K}^{+}$challenge. A moderate increase in $\left[\mathrm{K}^{+}\right]_{\mathrm{o}}$ opens Kir channels and produces a sustained hyperpolarization (Figure 2-2A). Simulations show jumps of $V_{m}$ from a depolarized to a hyperpolarized potential when $\left[\mathrm{K}^{+}\right]_{\mathrm{o}}$ increases past a critical concentration level, in agreement with an "all or none" $\mathrm{V}_{\mathrm{m}}$ response when increasing $\left[\mathrm{K}^{+}\right]_{\mathrm{o}}$ in cultured ECs [87]. The critical concentration for the hyperpolarizing $V_{m}$ jump depends

on $\mathrm{G}_{\mathrm{bg}} / \overline{\mathrm{G}}_{\mathrm{Kir}}$ ratio, as can be observed in solid vs. dashed blue traces (corresponding to 
solid vs. dashed green arrows in Figure 2-2 E). Decreasing $\left[\mathrm{K}^{+}\right]_{\mathrm{o}}$ back to resting levels can either return the cEC to the depolarized resting potential (solid red line), or leave the cell at a hyperpolarized potential (dashed red line). This behavior is attributed to the presence of two bifurcation points, i.e. saddle-node bifurcations, yielding a system that can exhibit hysteresis during a $\mathrm{K}^{+}$challenge/washout cycle and bistability within a $\mathrm{K}^{+}$concentration window (Figure 2-2 B-E). Small biological variability in channel densities will yield a binomial distribution of hyperpolarized and depolarized isolated ECs as seen in experimental studies $[87,90]$.

\subsection{2 cECs can have a bistable $V_{m}$}

The stability diagram in Figure 2-2 E summarizes the system's dynamic behavior as we move around in the parameter space. The diagram depicts the $\mathrm{K}^{+}$concentration window for bistability as the ratio of $\mathrm{G}_{\mathrm{bg}} / \overline{\mathrm{G}}_{\mathrm{Kir}}$ changes. As $\left[\mathrm{K}^{+}\right]_{\mathrm{o}}$ increases or $\mathrm{G}_{\mathrm{bg}} / \overline{\mathrm{G}}_{\mathrm{Kir}}$ decreases, the threshold $V_{m}$ (unstable steady-state; open circle in Figure 2-2 C) gets closer to the depolarized steady state (the solid red circle at more depolarized potentials). This lowers the $\Delta \mathrm{V}_{\mathrm{m}}$ threshold (and the corresponding current threshold) for jumping from the depolarized to the hyperpolarized steady-state, while increases the $\Delta \mathrm{V}_{\mathrm{m}}$ threshold for transition in the opposite direction. We separate the bistability region in hyperpolarization favoring (blue) and depolarization favoring (red) areas, based on the magnitude of $\Delta \mathrm{V}_{\mathrm{m}}$ thresholds required for transition towards the one or the other direction (Appendix B2). cECs can thus be classified into one of four groups according to their Kir conductance relative to the total transmembrane conductance: cells that have a single depolarized 
(yellow region) or hyperpolarized (grey region) steady-state, depolarization favoring (red region), or hyperpolarization favoring (blue region) bistable cells (Figure 2-2 E).

Studies have previously provided evidence for Kir-mediated bistable membrane potentials in ECs and SMCs [90, 98]. We examined whether the Kir current density in cECs is sufficient to create a bistable system at physiological $\left[\mathrm{K}^{+}\right]_{\mathrm{o}}$. Figure $2-2 \mathrm{~F}$ shows the summary data for the whole cell patch clamp recordings of enzymatically isolated cECs from mouse brain slices $[30,45]$ to determine the $\mathrm{Ba}^{2+}$ sensitive $\left(\mathrm{G}_{\mathrm{Kir}}\right)$ and insensitive $\left(\mathrm{G}_{\mathrm{bg}}\right)$ transmembrane conductances. Current densities $\left(\overline{\mathrm{G}}_{\mathrm{Kir}}=0.18 \pm 0.1 \mathrm{nS} / \mathrm{mM}^{0.5} ; \mathrm{G}_{\mathrm{bg}}=0.06 \pm 0.04\right.$ $\mathrm{nS}(\mathrm{n}=24))$ place cECs within/near the predicted bistability region at physiological $\left[\mathrm{K}^{+}\right]_{\mathrm{o}}$ (Refer to Appendix B2 for the effect of model parameters on predicted $\mathrm{V}_{\mathrm{m}}$ bistability window). Note that in this chapter we synonymously refer to as $\mathrm{G}_{\mathrm{Kir}}$ as the Kir channel conductance at $3 \mathrm{mM}$ of $\left[\mathrm{K}^{+}\right]_{0}$; unless stated otherwise. Dynamic regulation of $\mathrm{G}_{\mathrm{Kir}}$ [45] and/or $\left[\mathrm{K}^{+}\right]_{\mathrm{o}}$, and the level of inward rectification through the action of inhibitors like polyamines and $\mathrm{Mg}^{2+}$ [99], can change the percentage of bistable cells in a population of cECs. Overall, theoretical analysis and experimental data suggest that the presence of a dominant Kir current in cECs can create a "bistable switch" in $V_{m}$, a characteristic of electrical excitability and a necessary condition for regenerative, action potential-like propagation of electrical signals [100]. 

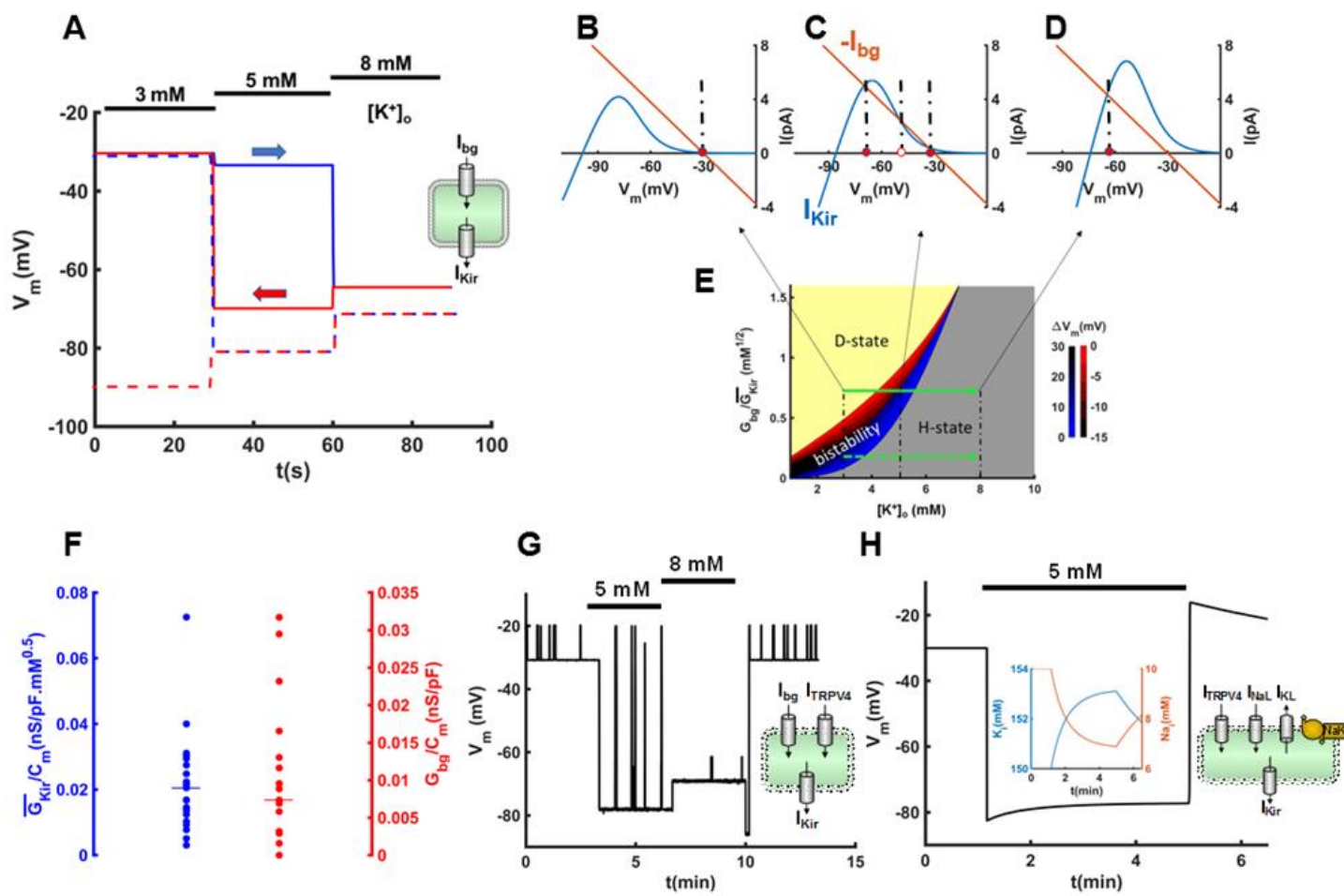

Figure 2-2 Single cEC response to modest elevations of $\left[\mathrm{K}^{+}\right]_{\mathrm{o}}$. (A) Simulated $\mathrm{V}_{\mathrm{m}}$ responses (Model A) to step increases (blue lines) and decreases (red lines) in $\left[\mathrm{K}^{+}\right]_{\mathrm{o}}$ show jumps between hyperpolarized and depolarized $\mathrm{V}_{\mathrm{m}}$ and hysteresis. Solid and dotted traces correspond to $\mathrm{G}_{\mathrm{bg}} / \overline{\mathrm{G}}_{\mathrm{Kir}}$ ratios of 0.7 and 0.3 , respectively. (B-E) Stability analysis: representative $\mathrm{I}_{\mathrm{Kir}}$ and $\left(-\mathrm{I}_{\mathrm{bg}}\right)$ are depicted as a function of $\mathrm{V}_{\mathrm{m}}$ at increasing $(3,5,8 \mathrm{mM})$ $\left[\mathrm{K}^{+}\right]_{\mathrm{o}}$. Intersection points denote steady-states (stable: solid circles, unstable: open circle) where the total current is zero. (B) At low $\left[\mathrm{K}^{+}\right]_{\text {o }}$ the outward Kir current is small and the cell rests at a depolarized $\mathrm{V}_{\mathrm{m}}$. (C) Increasing $\left[\mathrm{K}^{+}\right]_{\mathrm{o}}$ leads to a rightward and upward shift of the $\mathrm{I}_{\mathrm{Kir}}$ curve as a result of the increase in $\mathrm{G}_{\mathrm{Kir}}$ and $E_{K}$. As the $\left[\mathrm{K}^{+}\right]_{\mathrm{o}}$ increases past a critical concentration the system undergoes a saddle-node bifurcation and three steady-states emerge. The depolarized and hyperpolarized stable steady-states (solid red circles) are separated by an unstable steady state in between (threshold $V_{m}$, open circle). (D) Further increase in $\left[\mathrm{K}^{+}\right]_{\mathrm{o}}$ drives the system past a second saddle-node bifurcation. The presence now of only one hyperpolarized steady-state will force the cell to jump to a hyperpolarized $V_{m}$ close to $E_{K}$. (E) Stability diagram shows the parameter space where bistability (depicted in $\mathrm{C}$ ) exists. Blue/Red color maps show $\Delta \mathrm{V}_{\mathrm{m}}$ thresholds in bistable regions favoring transitions to the hyperpolarized/depolarized state. F) Patch clamp recordings of isolated cECs with $6 \mathrm{mM}$ and $60 \mathrm{mM}\left[\mathrm{K}^{+}\right]_{\mathrm{o}}$ bath concentrations $(\mathrm{n}=24)$, before and after Kir inhibition with $\mathrm{Ba}^{2+}$, were used to determine $\overline{\mathrm{G}}_{\mathrm{Kir}}=0.18+0.1 \mathrm{nS} / \mathrm{mM}^{0.5}\left(\mathrm{Ba}^{2+}\right.$ sensitive) and $\mathrm{G}_{\mathrm{bg}}=0.06+0.04 \mathrm{nS}\left(\mathrm{Ba}^{2+}\right.$ insensitive) condutances (G) Minimal model (Model B in Figure 2-1) examines Kir-TRPV4 dynamics. Stochastic openings of a single TRPV4 channel yield transient depolarizations. At intermediate $\left[\mathrm{K}^{+}\right]_{0}$, transient fluctuations between two polarization levels are predicted as the system crosses the saddle node bifurcation. $\mathrm{V}_{\mathrm{m}}$ fluctuations are dampened at higher levels of stimulation as the TRPV4 current is not sufficient to transition the system to the depolarized state. (H) Minimal model (Model C in Figure 2-1) of a cEC examines Kir-NaK dynamics. Increasing $\left[\mathrm{K}^{+}\right]_{\mathrm{o}}$ activates low affinity $\mathrm{NaK}$ pump isoforms producing transient hyperpolarization, $\left[\mathrm{Na}^{+}\right]_{i}$ depletion and a rebound depolarization after stimulus termination. Parameter values used for the simulations performed in this figure is summarized in Table 2-1.

\subsubsection{Nak pump and TRPV4 mediate transient $V_{m}$ responses during $A \mathrm{~K}^{+}$challenge}

Stochastic openings of TRPV4 can produce transient depolarizations in $\mathrm{V}_{\mathrm{m}}$. At moderate

levels of $\mathrm{K}^{+}$stimulation, the TRPV4 bursting activity can drive the system past the 
bifurcation points (i.e. transitioning a cEC from grey to yellow), producing large transient $\mathrm{V}_{\mathrm{m}}$ jumps in simulations using model $\mathrm{B}$ and model D (Figure 2-2 $\mathrm{G}$ and Appendix C2, respectively). These results are in line with experimental data where isolated ECs and arterioles exhibit fluctuations between two polarization levels upon stimulation with intermediate $\mathrm{K}^{+}$concentrations $(\sim 5-8 \mathrm{mM})[87,98,101]$. This experimentally observed instability suggests a bistable system and can be reproduced in silico when accounting for the stochastic opening of TRPV4. The stochastic $V_{m}$ fluctuations are dampened with further increasing $\left[\mathrm{K}^{+}\right]_{\mathrm{o}}$ (i.e. $\geq 8 \mathrm{mM}$ ) as the TRPV4 current is not sufficient to drive the cell past the threshold $\mathrm{V}_{\mathrm{m}}$ (Figure 2-2G and Appendix C2, panel D).

$\mathrm{A} \mathrm{K}^{+}$challenge can also transiently increase the activity of $\mathrm{NaK}$ pumps (i.e. particularly of low $\mathrm{K}^{+}$affinity isoforms; $\mathrm{NaK}_{\alpha 2}, \mathrm{NaK}_{\alpha 3}$ ), producing a transient hyperpolarization [102]) (Figure 2-2 H). In representative simulations using minimal (Model C) or detailed (Model D, Appendices A2 and C2) models, transient NaK-mediated hyperpolarization could be reproduced and was typically followed by a rebound depolarization after termination of a prolonged $\mathrm{K}^{+}$stimulation (resulting from reduced pump activity due to intracellular $\mathrm{Na}^{+}$ depletion, Figure 2-2 H inset). The TRPV4 and NaK-induced $V_{m}$ transients can facilitate transitions between hyperpolarized and depolarized $\mathrm{V}_{\mathrm{m}}$, in bistable cECs during an increase in $\left[\mathrm{K}^{+}\right]_{\mathrm{o}}$ or upon its return to resting levels (Figure 2-3 C).

\subsubsection{Bistable cells amplify electrical signals and promote hyperpolarizing jumps in capillary segments}

Action potential (AP) firing, as described by Hodgkin-Huxley type dynamics, is due to the voltage dependency of the $\mathrm{Na}^{+}$channel's $\left(\mathrm{Na}_{\mathrm{v}}\right)$ activation gate that initially creates (i.e. prior to the $\mathrm{Na}_{\mathrm{v}}$ inactivation or the delayed voltage gated potassium channel $(\mathrm{Kv})$ 
activation) a bistable $\mathrm{V}_{\mathrm{m}}$ (Appendix D2). Thus, from a dynamics point of view, $\mathrm{V}_{\mathrm{m}}$ bistability allows for the "all-or-none" response in AP and the regenerative propagation of a depolarizing front along neurons. Similarly, Kir-induced bistability can facilitate excitability and regenerative propagation of electrical signals along the endothelium [100]. In Figure 2-3 A we formulated a model of a capillary segment by placing cECs in series and coupled them through gap junctions with a cell-to-cell electrical resistance, $R_{\mathrm{gg}}$. Model simulations show that at low Kir current densities, a local $\mathrm{K}^{+}$stimulus produces a small local hyperpolarization that spreads passively and attenuates along the capillary segment with a length constant of signal attenuation, $\lambda=\sqrt{r_{m} / r_{i}}$ (where $r_{i}=R_{g j} / L_{E C}$ and $r_{m}=R_{m} L_{E C}$ are the axial membrane resistivity per unit length, and $L_{E C}$ and $R_{m}$ are the cEC length and membrane resistance, respectively). At high Kir densities, model predicts significant local hyperpolarization (stimulated cells jump close to $E_{K}$ ) that spreads without attenuation (i.e. excitable/regenerative system). The latter case is possible when cECs are bistable and hyperpolarization favoring (i.e. blue region in Figure 2-2 E).

The passive system resets following stimulus termination in our capillary model (Figure 2-3 B), i.e. $V_{m}$ returns to its basal value upon removal of the stimulus. Removing the stimulus could not reset $\mathrm{V}_{\mathrm{m}}$ in the excitable system as the local bistable cells did not depolarize by the return of $\left[\mathrm{K}^{+}\right]_{\mathrm{o}}$ to resting levels, due to hysteresis (Figure 2-2 A dotted line). Furthermore, as theoretical analysis in [100] indicates, an infinitely long capillary (i.e. capillary length $(\mathrm{L})>>\lambda$ ) with identical and bistable ECs can promote regenerative conduction of either a hyperpolarizing (blue ECs) or a depolarizing (red ECs) front, but not both at the same time. Thus, a local return to the resting depolarized $\mathrm{Vm}$, cannot propagate 
in our model capillary under the conditions of Figure 2-3 A,B. Similar to an AP, delayed inactivation/activation of channels may be required to depolarize the capillary back to resting $\mathrm{V}_{\mathrm{m}}$.

\subsubsection{TRPV4 and NaK can facilitate system return following stimulus termination} Model simulations in Figure 2-3C suggest that the presence of TRPV4 channels and/or the activity of the NaK pump can provide a resetting mechanism for the excitable capillary layer in Figure 2-3 A,B. Basal TRPV4 channel activity (at levels that have been observed experimentally [46]), was capable of turning back the "bistable switch" in a hyperpolarized capillary segment (Appendix E2). The depolarizing effect of stochastic TRPV4 transients along the capillary segment enable depolarization to resting levels after stimulus termination (when the current threshold for transitioning to the depolarized $\mathrm{V}_{\mathrm{m}}$ is reduced). The NaK-induced transient hyperpolarization/depolarization during stimulus initiation/termination (Figure 2-2 H) promotes local, stimulated bistable cells to jump to a hyperpolarized/depolarized potential, respectively (Appendix E2). TRPV4 and NaK activity can, thus, conditionally facilitate (i.e. depending on the required threshold current) the return of the capillary to the depolarized resting potential upon stimulates termination. More robust resetting can be achieved by a progressive downregulation of Kir activity and/or upregulation of TRPV4 upon hyperpolarization as observed experimentally during plasma membrane phosphatidylinositol 4,5-bisphosphate (PIP2) or ATP depletion [45, 46]. This would slowly transition cECs from hyperpolarization favoring (blue) during stimulus initiation to depolarization favoring (red or yellow), enabling capillary $\mathrm{V}_{\mathrm{m}}$ to return to resting potential and protect the system against a prolonged excitability (Appendix E2). 

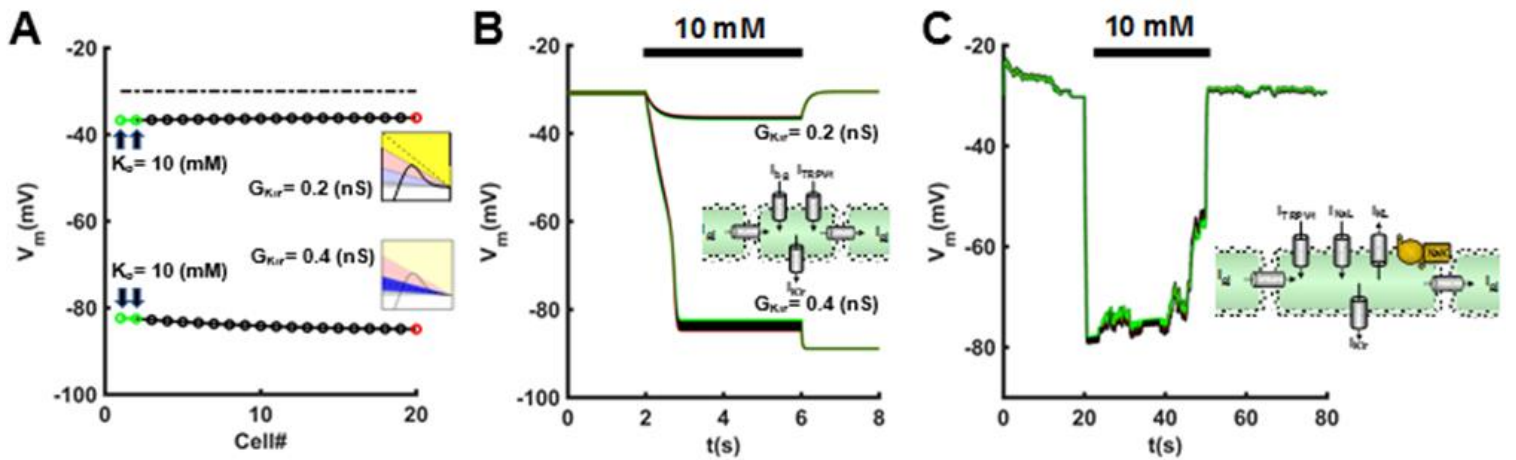

Long segment
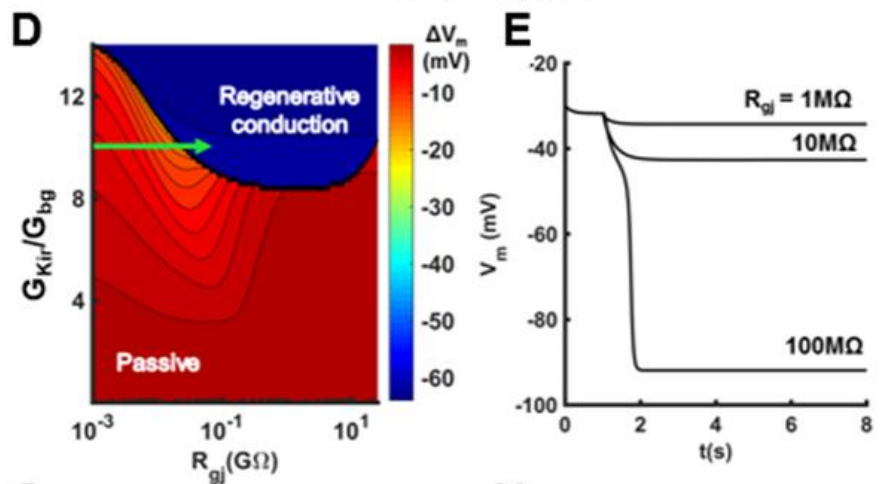

Short segment
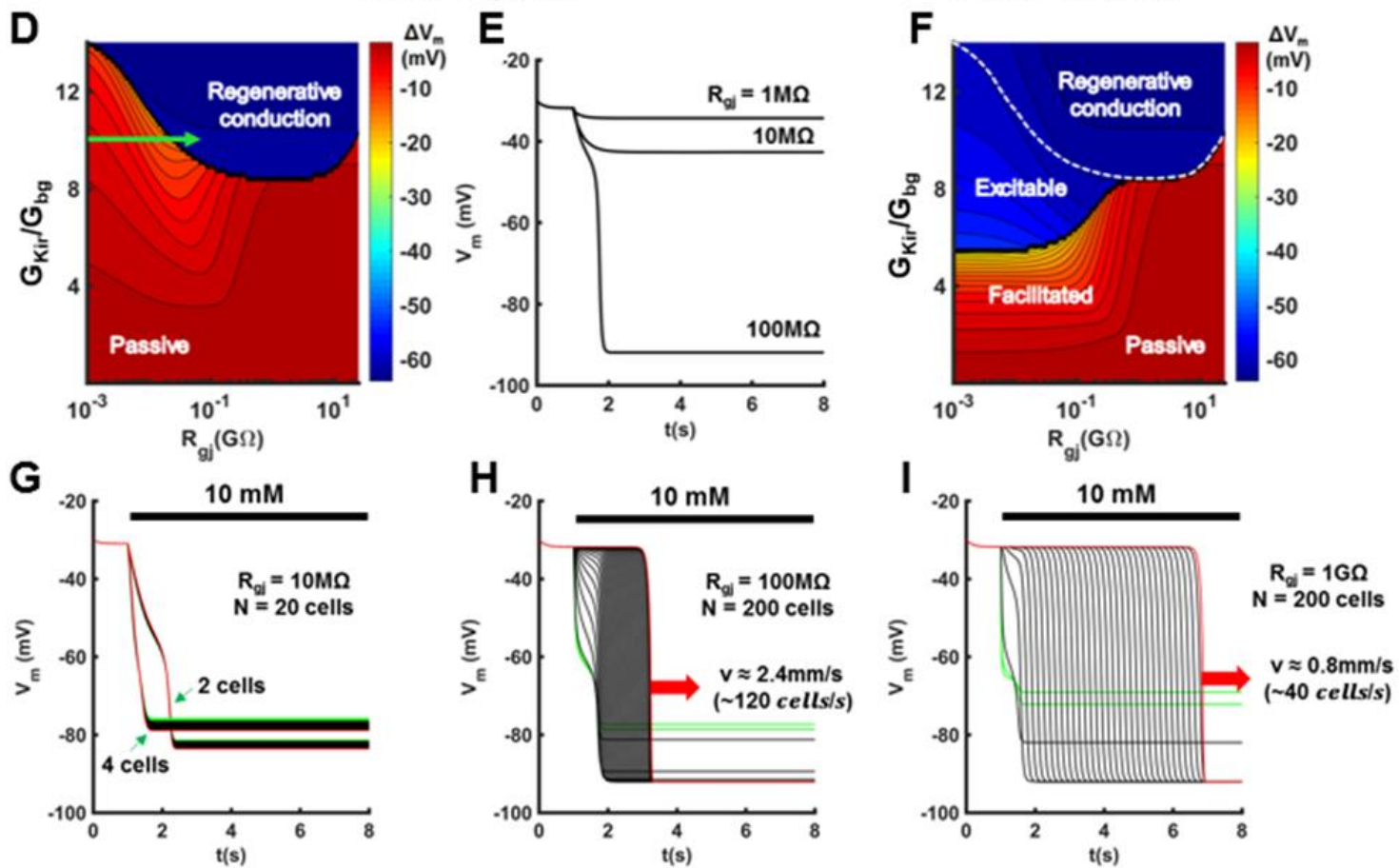

Figure 2-3 Electrical conduction in a capillary segment. cECs (Model A) where coupled in series to form a model of a capillary segment. (A) An array of 20 axially connected and identical cECs are stimulated at the left end ( $\mathrm{n}=2$ cells) with $10 \mathrm{mM} \mathrm{K} \mathrm{K}^{+}$and $\mathrm{V}_{\mathrm{m}}$ responses are depicted at rest (dashed line) and following stimulation for $\mathrm{G}_{\mathrm{Kir}}=0.2 \mathrm{nS}$ and $0.4 \mathrm{nS}$ and $\mathrm{R}_{\mathrm{gj}}=10 \mathrm{M} \Omega$. Bistable $\mathrm{cECs}\left(\mathrm{G}_{\mathrm{Kir}}=0.4 \mathrm{nS}\right.$; blue cECs) can produce significantly higher local hyperpolarization which propagates without attenuation. (B) Time course of the responses in (A). System resets after stimulus end when low levels of $\mathrm{G}_{\mathrm{Kir}}$ are assumed $\left(\mathrm{G}_{\mathrm{Kir}}=0.2 \mathrm{nS}\right.$; yellow cECs), but it does not when higher $\mathrm{G}_{\mathrm{Kir}}$ (blue cECs) promote a hyperpolarizing jump upon stimulation. (C) TRPV4 and NaK (Model C) can facilitate resetting in an excitable system $\left(\mathrm{G}_{\mathrm{Kir}}=0.4 \mathrm{nS}\right)$. (D-F) Level of distal $\left(400 \mu \mathrm{m}\right.$ from stimulus site) hyperpolarization $\left(\Delta \mathrm{V}_{\mathrm{m}}\right)$ color-coded for a long $(\mathrm{N}=200$ cells) $(\mathrm{D}, \mathrm{E})$ and a short $\left(\mathrm{N}=20\right.$ cells) (F) capillary segment. Depending on $\mathrm{G}_{\mathrm{Kir}}$ and $\mathrm{R}_{\mathrm{g}}$, passive conduction, facilitated hyperpolarization, or excitability with or without regenerative propagation of a hyperpolarizing front is predicted. In the long capillary segment, increasing $\mathrm{R}_{\mathrm{gj}}$ (arrow in Figure 2-3 $\mathrm{D}$ ) can increase distal hyperpolarization by promoting regenerative conduction (E). (G) Time course of the hyperpolarizing jump in a short, well-coupled segment upon stimulation of 2 or 4 cells at one end. (H,I) Regenerative propagation of the hyperpolarizing front in a long segment $\left(\mathrm{N}=200\right.$ cells) with $\mathrm{R}_{\mathrm{gj}}$ of $100 \mathrm{M} \Omega(\mathrm{H})$ and $1 \mathrm{G} \Omega$ (I). The rest of the parameters used for the simulations performed in this figure is summarized in Table 2-1 


\subsubsection{Weak intercellular coupling promoting regenerative electrical conduction}

Whether a $\mathrm{K}^{+}$challenge can produce a hyperpolarizing jump (i.e. whether capillary is excitable) depends on the ratio of $\mathrm{G}_{\mathrm{Kir}} / \mathrm{G}_{\mathrm{bg}}$, the stimulus strength (i.e. number of cells stimulated), and the coupling between neighboring cECs (Figure 2-3 D,F). Interestingly, the minimum required $\mathrm{G}_{\mathrm{Kir}}$ for excitability decrease as $\mathrm{R}_{\mathrm{gj}}$ increases under the conditions of Figure 2-3 D (i.e., long segment of cECs where $L>>\lambda$ ). This creates a paradox where for a given level of stimulation and Kir activity, significant local and distal hyperpolarization can be achieved only if ECs are poorly coupled (green arrow in Figure 2-3 D and traces in Figure 2-3 E) [100]. Model predicts that electrical signals can propagate along the endothelium even if $R_{\mathrm{gj}}$ is in the $\mathrm{G} \Omega$ range (compared to the $\mathrm{R}_{\mathrm{gj}}$ in the $\mathrm{M} \Omega$ range that enables robust but passive propagating responses in arteries $[94,95])$. This counterintuitive result is attributed to the increased sensitivity of the $\mathrm{cECs}$ to $\mathrm{K}^{+}$with higher $\mathrm{R}_{\mathrm{gj}}$ (i.e. current loss to neighboring cells is reduced and opening of Kir channels induces a larger local change in $\mathrm{V}_{\mathrm{m}}$ ) that allows for local hyperpolarization above the $\Delta \mathrm{V}_{\mathrm{m}}$ threshold for turning

the bistable switch on. Once $V_{m}$ passes the threshold value, the cEC jumps to a hyperpolarized potential and drives hyperpolarization of its neighboring cell, causing a domino-like effect along the capillary (regenerative conduction), analogous to the propagation of APs in neurons.

\subsubsection{Capillary length, coupling resistance and amplification of hyperpolarization} Simulations in short (relative to $\lambda$ ) capillary segments revealed different $V_{m}$ dynamics (Figure 2-3 F) compared to long segments (Figure 2-3 D). Significant hyperpolarization can be achieved with lower $\mathrm{G}_{\mathrm{Kir}}$, and $\mathrm{R}_{\mathrm{gj}}$ in the $\mathrm{M} \Omega$ range. Stimulation of a few cECs and the resulting small initial hyperpolarization is amplified by the opening of Kir channels 
along the length of the isolated and relatively well-coupled capillary segment. This Kirmediated amplification, results in higher levels of hyperpolarization (facilitated hyperpolarization) as has been documented in experiments [103] and the fidelity of the response increases with the increase in Kir density/activity. Further increase in $\mathrm{G}_{\mathrm{Kir}}$ above a threshold value (but below what is required for regenerative conduction in Figure 2-3 D) promotes a hyperpolarizing jump in $\mathrm{V}_{\mathrm{m}}$, upon $\mathrm{K}^{+}$stimulation. This Kir-mediated segment excitability differs from the regenerative conduction described above by the absence of a propagating hyperpolarizing front, i.e. the last cEC jumps to the hyperpolarize $V_{m}$ before the stimulated cECs (crossover of the red-green traces in Figure 2-3 G), and by its dependence on capillary size. Stimulation of a sufficient number of cECs may allow such a spatially-confined excitability in a capillary network (Figure 2-5). Thus, depending on length, stimulus strength and channel densities, stimulation of a capillary segment may result in a) a relatively small local $V_{m}$ change that propagates passively and dissipate with distance (passive system), b) a more pronounced local and distal hyperpolarization as the conducted signal is facilitated/amplified by the opening of Kir channels in neighboring cells (facilitated conduction), or c) a segment excitability with or without the regenerative propagation of a hyperpolarizing front.

\subsubsection{Conduction velocity}

In a capillary with passive characteristics (i.e. constant $R_{m}$ ), the conduction velocity $(v)$ is determined by the ratio of $\lambda$ to the characteristic time constant $\tau=C_{m} R_{m}$ (i.e. $v$ is in the

order of $\frac{\lambda}{\tau}=\frac{L_{E C}}{C_{m} \sqrt{r_{i} r_{m}}}$ ) which governs the response time of a distal cell upon local stimulation. In Figure 2-3 G-I we examine the conduction velocity in capillary segments 
under conditions that promote the opening of distal Kir channels (i.e. non-passive conductions). In short capillary segments $\left(\mathrm{L}=200 \mu \mathrm{m} ; \mathrm{R}_{\mathrm{gj}}=10 \mathrm{M} \Omega ; \frac{\lambda}{\tau}=6 \mathrm{~mm} / \mathrm{s} ; \tau=130 \mathrm{~ms}\right)$, distal cells respond fast upon $\mathrm{K}^{+}$stimulation (Figure 2-3 G) and detectable $\mathrm{V}_{\mathrm{m}}$ changes are predicted at the distal end within tens of milliseconds, which is close to the estimated $\frac{\lambda}{\tau}$ ratio. Even though cECs in the short and well coupled capillary segment respond nearly simultaneously to the potassium-stimulus, the system reaches its steady-state value with a delay of more than a second (i.e., the rate of $V_{m}$ change is drastically slower than what would have been predicted under passive conditions $(\tau=130 \mathrm{~ms}))$. This delay is significantly reduced, however, when more cells are stimulated (compare 2 vs. 4 cell stimulation in Figure 2-3 G). This behavior suggests that the response dynamics of distal cECs are not limited by the charging of membrane capacitance, but rather by the rate of progressive Kir channel recruitment as $\mathrm{V}_{\mathrm{m}}$ hyperpolarizes. Experimental data in small isolated capillary networks (Figure 2-4 A) demonstrate such characteristics, which can potentially provide evidence for a Kir-mediated amplification of the propagating electrical signal.

In long capillary segments $\left(\mathrm{L}=4000 \mu \mathrm{m} ; \mathrm{R}_{\mathrm{gj}}=100 \mathrm{M} \Omega-1 \mathrm{G} \Omega ; \frac{\lambda}{\tau}=2-0.6 \mathrm{~mm} / \mathrm{s}\right)$, distal cells will respond with a delay, reflecting the delayed arrival of the hyperpolarizing front, followed by a fast transition to a hyperpolarized $V_{m}$ (Figure 2-3 H,I). The conduction velocity of the hyperpolarizing front is $v \approx 40 \mathrm{cECs} / \mathrm{s}$ or $\approx 0.8 \mathrm{~mm} / \mathrm{s}$ for $\mathrm{R}_{\mathrm{gj}}$ of $1 \mathrm{G} \Omega$ (Figure 2-3 I) and increases to $120 \mathrm{cECs} / \mathrm{s}, \approx 2.4 \mathrm{~mm} / \mathrm{s}$, if $\mathrm{R}_{\mathrm{gj}}=100 \mathrm{M} \Omega$ (Figure $2-3 \mathrm{H}$ ). The latter is still three orders of magnitude less than typical conduction velocities in unmyelinated 
neuronal axons, owing to approximately three orders of magnitude larger $r_{m}$ and $r_{i}$ of capillary segment to those of axons.

\subsubsection{Evidence for regenerative hyperpolarization in the brain microcirculation - an inverted sustained action potential}

We have recently provided ample evidence that elevation of $\mathrm{K}^{+}$around capillaries transmits electrical signals to upstream arterioles [30]. Picospritzing $10 \mathrm{mM} \mathrm{K}^{+}$onto a few capillary ends in an ex vivo preparation of a PA with attached capillaries (Cap-PA) (Figure 2-4 A inset) caused significant hyperpolarization (up to $30 \mathrm{mV}$ ) and maximal dilation of the PA hundreds of microns away from the stimulus site (Figure 2-4 A). However, the magnitude of outward Kir current in cECs (often below the limit of detection in patch clamp experiments) suggests that $\mathrm{K}^{+}$stimulation of a few cECs can only generate up to a few pico amperes of hyperpolarizing current. By comparison, equivalent conducted responses in small arterioles typically require several nano amperes of stimulating current $[94,104$, 105]. This disparity in stimulatory current requirement cannot be explained by differences in size or the number of cells between arteries and capillaries. How does the stimulation of a few cECs produce robust dilations in upstream feeding arteries?

We formulated a model capillary-PA network that resembles the Cap-PA preparation (Figure 2-4 A inset) and examined the effect of increasing $\left[\mathrm{K}^{+}\right]_{\mathrm{o}}$ on cECs located on one end of the capillary branch ( $15 \%$ of total cECs in the network). Simulations assume a heterogeneous population of cECs with variable number of active Kir channels to account for biological variability. As the mean $\mathrm{G}_{\mathrm{Kir}}$ increases, the majority of the cell population shifts from yellow, to red, to blue (as shown in the respective pie charts in panels $\mathrm{C}-\mathrm{E}$ ). A heterogeneous capillary network behaves passively when most cells are yellow and 
remains insensitive to $\mathrm{K}^{+}$stimulation (Figure 2-4 C). This response is comparable to that of a capillary network without Kir channels $\left(\mathrm{EC} \mathrm{Kir}^{---}\right)$which is locally stimulated with an equivalent current injection (Figure 2-4 B). Thus, model predicts a limited hyperpolarization of the feeding PA (black traces) under passive conditions, significantly below the observed 20-30 $\mathrm{mV}$ arteriolar response in the experiments. The effect from an equivalent local stimulation in vivo is expected to be even lower if current disperses in both upstream and downstream directions and to every connected side branch. Thus, comparison of ex vivo and in silico data suggests the presence of an amplification mechanism of the capillary-initiated electrical signaling, intrinsic to the microvascular network.
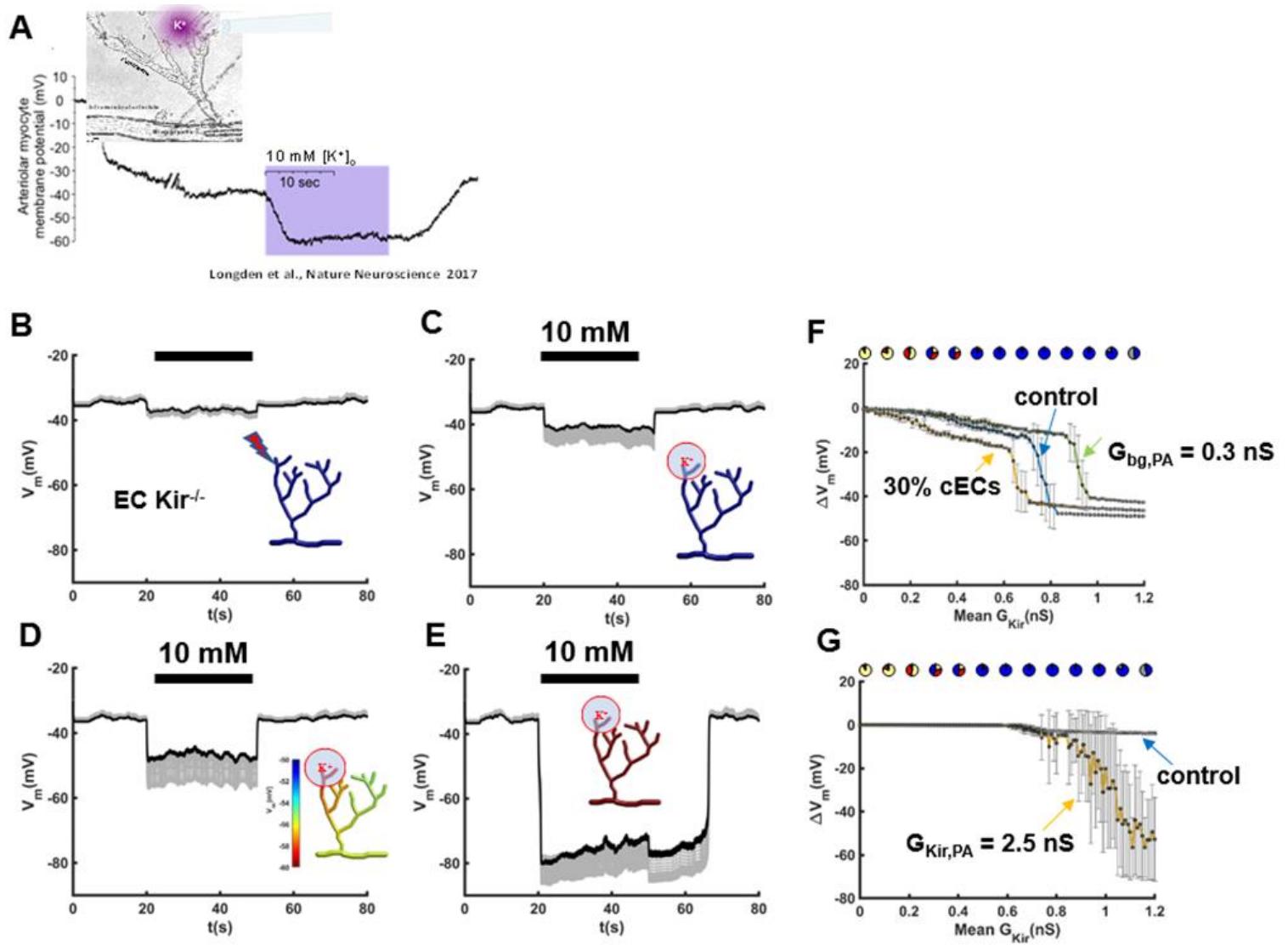

Figure 2-4 Ex vivo Capillary-PA preparation. (A) Experimental data showing smooth muscle $\mathrm{V}_{\mathrm{m}}$ recording in an isolated and pressurized PA with the capillary network attached (inset) when $10 \mathrm{mM} \mathrm{K}^{+}$is picospritzed onto the capillary ends. (B-E) Model simulation in a corresponding microvascular network (inset) that contains $45 \mathrm{cECs}$ and a $300 \mu \mathrm{m}$ PA consisting of 45 coupled EC-SMC units (15 longitudinal ECs with a 3 
by 3 connection between ECs and SMCs, i.e. each PA EC is connected to 3 SMCs and vice versa). Each cEC is represented with Model C. A localized region is simulated by injecting $4 \mathrm{pA}$ of current in (B) (to simulate the response of the system during EC $\mathrm{Kir}^{-/-}$), or via application of $10 \mathrm{mM} \mathrm{K}{ }^{+}$on $7 \mathrm{cECs}$ in $(\mathrm{C}-\mathrm{E})$ at one end of the network (highlighted in the figure inset). In panels $(\mathrm{C}-\mathrm{E})$ the mean $\mathrm{cEC} \mathrm{G}_{\mathrm{Kir}}$ is progressively increased from 0.2 , to 0.4 and $0.6 \mathrm{nS}$, respectively. In simulations performed for panel $(\mathrm{E})$, the activity of Kir and TRPV4 of cECs is dynamically regulated upon the start of $\mathrm{K}^{+}$stimulation (gradual decrease in Kir and gradual increase in TRPV4 with a time constant of 6 minutes, simulating the effect of PIP2 depletion on cEC activity [46]). (F-G) summary data for the predicted level of PA hyperpolarization (at $\sim 120 \mu \mathrm{m}$ away from the capillary PA junction) during a $\mathrm{K}^{+}$challenge in a relatively well-coupled $\left(\mathrm{R}_{\mathrm{gj}}=10 \mathrm{M} \Omega\right)$ and a poorlycoupled network $\left(R_{\mathrm{gj}}=1 \mathrm{G} \Omega\right)$, shown as mean \pm std. As the mean $\mathrm{G}_{\mathrm{Kir}}$ in cECs increases a progressive increase in the level of PA hyperpolarization is predicted before a hyperpolarizing jump to voltages close to $E_{K}$. For each mean $\mathrm{cEC}_{\mathrm{Kir}}$ level, 30 simulations are performed with standard deviation of $0.2 \mathrm{nS}$. (F) Leftmost trace $\left(\mathrm{n}=15 \mathrm{cECs}(\sim 30 \%)\right.$ stimulated; $\mathrm{G}_{\mathrm{bg}, \mathrm{PA}}=0.2 \mathrm{nS}$ and $\left.\mathrm{G}_{\mathrm{Kir}, \mathrm{PA}}=0.65 \mathrm{nS}\right) ;$ middle trace $(\mathrm{n}=7 \mathrm{cECs}(\sim 15 \%)$ stimulated; $\mathrm{G}_{\mathrm{bg}, \mathrm{PA}}=0.2 \mathrm{nS}$ and $\left.\mathrm{G}_{\mathrm{Kir}, \mathrm{PA}}=0.65 \mathrm{nS}\right)$; rightmost trace $\left(\mathrm{n}=7 \mathrm{cECs}\right.$ stimulated; $\mathrm{G}_{\mathrm{bg}, \mathrm{PA}}=0.3 \mathrm{nS}$ and $\mathrm{G}_{\mathrm{Kir}, \mathrm{PA}}=0.65 \mathrm{nS}$ ). In all simulations of this panel, $\mathrm{R}_{\mathrm{gj}}=10 \mathrm{M} \Omega$. Pie charts indicate the relative distribution of cECs based on the classification in Figure 2-2.E (G) Left trace ( $\mathrm{n}=7$ cECs stimulated; $\mathrm{G}_{\mathrm{bg}, \mathrm{PA}}=0.2 \mathrm{nS}$ and $\left.\mathrm{G}_{\mathrm{Kir}, \mathrm{PA}}=0.65 \mathrm{nS}\right)$; right trace $\left(\mathrm{n}=7 \mathrm{cECs}\right.$ stimulated; $\mathrm{G}_{\mathrm{bg}, \mathrm{PA}}=0.2 \mathrm{nS}$ and $\left.\mathrm{G}_{\mathrm{Kir}, \mathrm{PA}}=2.5 \mathrm{nS}\right)$. In all simulations of this panel $\mathrm{R}_{\mathrm{gj}}=1 \mathrm{G} \Omega$. The rest of the parameters for the simulations in this figure are the same as in Table $2-1$.

When a higher Kir density is assumed (mean $\mathrm{G}_{\mathrm{Kir}}=0.5 \mathrm{nS}$ ), the majority of cECs become bistable, and the $\mathrm{K}^{+}$challenge produces a local hyperpolarization that is amplified by cECs along the capillary network (Figure 2-4 D,E). A network containing mostly bistable and hyperpolarization favoring (i.e. blue) cECs (Figure 2-4 E), produces significant local response upon stimulation $\left(\mathrm{V}_{\mathrm{m}}\right.$ jumps close to $\left.\mathrm{E}_{\mathrm{K}}\right)$ and the predicted level of $\mathrm{EC}$ hyperpolarization in the upstream PA is comparable to the experimentally observed SMC responses in Figure 2-4 A. Furthermore, the $\mathrm{V}_{\mathrm{m}}$ traces in Figure 2-4 E can account for the slow increase in $V_{m}$ hyperpolarization observed in Figure 2-4 A, as Kir channels are progressively recruited. Additionally, consistent with the observations in Figure 2-4 A, the return of the system to its resting $V_{m}$ in Figure 2-4 is delayed as the bistable system can stay at a hyperpolarize potential following stimulus termination until activity of a sufficient number of Kir channels is reached blow the threshold (upon PIP2 depletion) for turning the "bistable switch" off. At Kir activity levels, below the requirement for excitability, amplification of hyperpolarization (facilitated conduction) and a passive return (i.e. 
without the need of NaK, TRPV4 or dynamic changes in channel conductances) to resting levels following stimulus termination is observed (Figure 2-4 D).

Figure 2-4 F,G present summary data from simulations using the Cap-PA model as mean $\mathrm{G}_{\text {Kir }}$ varies from 0-1.2 nS. In a relatively well-coupled network $\left(\mathrm{R}_{\mathrm{gj}}=10 \mathrm{M} \Omega\right.$, Figure 2-4 F), sufficient Kir activity $\left(\mathrm{G}_{\mathrm{Kir}}=0.8 \mathrm{nS}\right)$ promotes a $\mathrm{V}_{\mathrm{m}}$ jump to values close to $\mathrm{E}_{\mathrm{K}}$ upon $\mathrm{K}^{+}$ stimulation $(10 \mathrm{mM})$. As the leakiness of the PA increases, or the number of stimulated cells decreases, higher Kir activity is required to promote significant PA hyperpolarization through network excitability. In a poorly coupled network (Figure 2-4 G, $\mathrm{R}_{\mathrm{gj}}=\mathrm{R}_{\mathrm{gj}, \mathrm{PA}}=1$ $\mathrm{G} \Omega$ ), $\mathrm{K}^{+}$stimulation of distal cECs can promote PA hyperpolarization when sufficient Kir densities allow for regenerative conduction along the capillary network and the PA. Collectively, simulation results suggest that even with conservative estimates for critical parameter values $\left(\mathrm{R}_{\mathrm{gj}}=10 \mathrm{M} \Omega, \mathrm{n}=15\right.$ cells $\left(30 \%\right.$ of total $\mathrm{cEC}$ stimulated), $\left.\mathrm{r}_{\mathrm{m}, \mathrm{PA}}=5 \mathrm{G} \Omega \mathrm{cm}\right)$ the observed PA SMC hyperpolarization in the experiments (up to $30 \mathrm{mV}$ ) cannot be explained unless the capillary initiated signal is significantly amplified by bistable ECs as it is conducted towards the PA.

\subsubsection{Binary vs graded responses of capillary modules to $\mathrm{K}^{+}$stimulus}

Simulations using realistic angioarchitectures allow us to relate ex vivo data to in vivo function. We used a small segment of an in vivo capillary network reconstruction from the vibrissa primary sensory cortex of a mouse (adapted from [96]) with its feeding PA and a draining parenchymal venule (PV) to examine how neuronal signals are integrated in the capillary network to produce hyperpolarization (dilation) of the feeding arteriole (Figure 2-5 A). Simulations assume a heterogeneous population of cECs with variable number of 
active Kir channels to account for biological variability and we examine the effect of increasing the mean $G_{K i r}$ in the capillary network to probe the system's ability to dynamically regulate Kir activity (i.e. through PIP2/ATP depletion [46]). Simulations in Figure 2-5 A show that stimulating a small number of cECs $(\sim 10-14 \%$ of total cECs, highlighted in white) with $10 \mathrm{mM} \mathrm{K}{ }^{+}$can hyperpolarize the feeding PA. Similar to the simulations of the ex vivo Cap-PA preparation in Figure 2-4 when the mean cEC $\mathrm{G}_{\mathrm{Kir}}$ is small (i.e. $0.1 \mathrm{nS}$; yellow cECs), a passive capillary network is insensitive to $\mathrm{K}^{+}$stimulation. As the mean $\mathrm{G}_{\mathrm{Kir}}$ (and the percentage of bistable cECs) increases, so does the sensitivity of the microvasculature to local $\mathrm{K}^{+}$stimulation. In a relatively well-coupled network $\left(\mathrm{R}_{\mathrm{g}}=50 \mathrm{M} \Omega\right)$, graded increases in PA hyperpolarization are predicted as the number of stimulated cECs increases (Figure 2-5 A, mean $\mathrm{G}_{\mathrm{Kir}}=0.4$ and $0.5 \mathrm{nS}$ ). Above a mean $\mathrm{G}_{\mathrm{Kir}}$ threshold $(>0.5 \mathrm{nS}$; where majority of cECs are blue), a highly sensitive capillary module allows maximum hyperpolarization when a sufficient number of cells are stimulated, and the module operates in a binary (on/off) mode. Summary results for the model network in A are presented in Figure 2-5 B. Depending on the level of Kir activity, the microvascular network can exhibit graded or "all-or-none" responses as the number of stimulated cells increase, and for a given stimulatory scenario, modulation of $\mathrm{G}_{\mathrm{Kir}}$ can regulate the fidelity of the response. In larger microvascular networks in vivo, graded responses can be achieved at the arteriole level by summation of binary inputs from multiple activated capillary network regions (Appendix F2). 


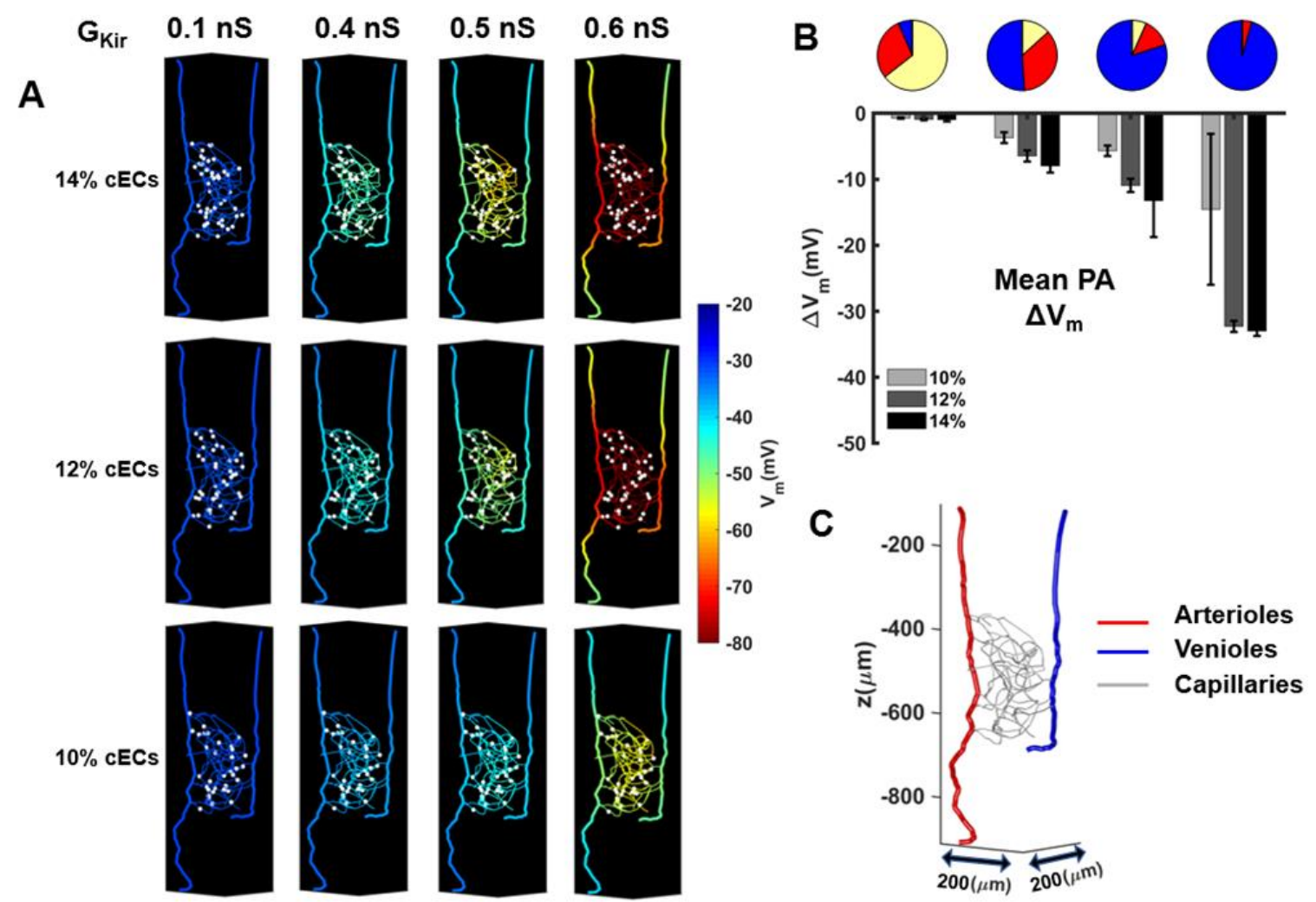

Figure 2-5 Binary and graded hyperpolarization of PAs in vivo. (A) Predicted $V_{m}$ hyperpolarization (color coded) in a capillary network reconstruction from the vibrissa primary sensory cortex of a mouse (adapted from [96]) with its feeding PA and draining venule, stimulated with $10 \mathrm{mM}$ of $\left[\mathrm{K}^{+}\right]_{\mathrm{o}}$ (stimulated cells are highlighted with white circles). A heterogeneous population of cECs with different Kir densities is assumed (normal distribution of $\mathrm{cEC} \mathrm{G}_{\mathrm{Kir}}$ with standard deviation of $0.2 \mathrm{nS}$ ). Simulations for different mean $\mathrm{cEC} \mathrm{G}_{\mathrm{Kir}}$, and different number of randomly selected cECs (as the percentage of total in the network) are presented. At low levels of $\mathrm{G}_{\mathrm{Kir}}(0.1 \mathrm{nS}$, first column) system is relatively insensitive to potassium stimulus and the number of stimulated cells. Increasing the mean $\mathrm{G}_{\mathrm{Kir}}$ level ( 0.3 and $0.4 \mathrm{nS}$, second and third columns) place more cECs in the bistable region, and hence the PA hyperpolarization is enhanced in a graded fashion with the increase in stimulation strength. Further increase in the $\mathrm{cEC}$ mean $\mathrm{G}_{\mathrm{Kir}}$ will make the capillary network highly sensitive to potassium stimulus (fourth column, $\mathrm{G}_{\mathrm{Kir}}=0.6 \mathrm{nS}$, majority of cells are hyperpolarization favoring), and a threshold number of stimulated cells will result in a hyperpolarization jump in the entire capillary network which in turn increases the level of PA hyperpolarization along the network (B) Summary data for the mean PA hyperpolarization in the network presented in (A) upon $10 \mathrm{mM}\left[\mathrm{K}^{+}\right]_{\mathrm{o}}$ stimulation of varying percentages of stimulated cECs for $\mathrm{R}_{\mathrm{gj}}=50 \mathrm{M} \Omega$ (30 trials). Pie charts show the distribution of cECs at mean $\mathrm{G}_{\mathrm{Kir}}$ levels of 0.1, 0.4, 0.5 and $0.6 \mathrm{nS}$, respectively. Grouped bar charts (shown as mean \pm std) show graded and binary responses in PA hyperpolarization as the number of stimulated cells increase for different values of mean $\mathrm{G}_{\mathrm{Kir}}$. (C) schematic of the network used for the simulations in panels A and B where cECs are in gray, PAs are in red, and PVs are in blue. For all simulations performed in this figure, each cEC is modeled using Model A, and PVs are assumed to have the same $\mathrm{G}_{\mathrm{bg}}$ and $\mathrm{R}_{\mathrm{gj}}$ as PAs, but lack Kir channels.

\subsubsection{Retrograde electrical signaling through capillaries and PAs}

We used larger realistic microvascular network adaptations to test whether a focal stimulation deep in the cortex can be conducted to the surface microcirculation for robust 
dilation and $\mathrm{CBF}$ increase. We examined if such propagation of electrical activity could be effectively carried through the PA and/or the capillary network. In simulations in Figure 2-6 A we stimulate a PA segment (100 $\mu \mathrm{m}$ in length), located in cortical layer IV or layer VI, with $10 \mathrm{mM} \mathrm{K}^{+}$. Under control conditions, we assume a relatively low Kir activity in the capillaries and in PAs (i.e $\mathrm{G}_{\mathrm{Kir}, \mathrm{PA}}=0.65 \mathrm{nS}, \mathrm{G}_{\mathrm{Kir}}=0.3 \mathrm{nS}$ ), the model predicts only a few $\mathrm{mV}$ of arteriolar hyperpolarization. Robust hyperpolarization of the PA and its feeding surface arteries require higher PA Kir densities to amplify the local stimulatory signal. The fidelity of local $\mathrm{K}^{+}$stimulation and the efficiency of electrical conduction along the PA were significantly affected by the level cEC coupling in surrounding capillaries; this is attributed to the dissipation of the electrical signal at capillary branching points that increases as $\mathrm{R}_{\mathrm{gj}}$ decreases. Results also demonstrate dependence on the location of focal stimulation. A higher capillary density in the deeper layers of the cortex leads to attenuated hyperpolarization and less efficient electrical conduction when the PA is stimulated in layer VI vs in layer IV (Figure 2-6 B). Despite the inhibitory effect of a capillary network with low Kir activity $\left(\mathrm{G}_{\mathrm{Kir}}=0.3 \mathrm{nS}\right)$ on the retrograde signaling through the PA, conditions could be identified $\left(\mathrm{G}_{\mathrm{Kir}, \mathrm{PA}}=2.4 \mathrm{nS} ; \mathrm{R}_{\mathrm{g}}=100 \mathrm{M} \Omega\right)$ that would promote PA excitability and significant hyperpolarization of the feeding surface arteries. Overall, simulation results suggest that significant upstream hyperpolarization upon focal PA stimulation requires sufficient Kir channel density to amplify the stimulatory current and a high coupling resistance at branching capillaries to limit the dissipation of the conducting current towards the capillary network. Simulations show that exposing surrounding $\mathrm{cECs}$ to $\mathrm{K}^{+}$, in addition to a direct stimulation of the PA segment, can increase the local stimulatory current and as a result, increase PA hyperpolarization (Figure 2-6 C). 

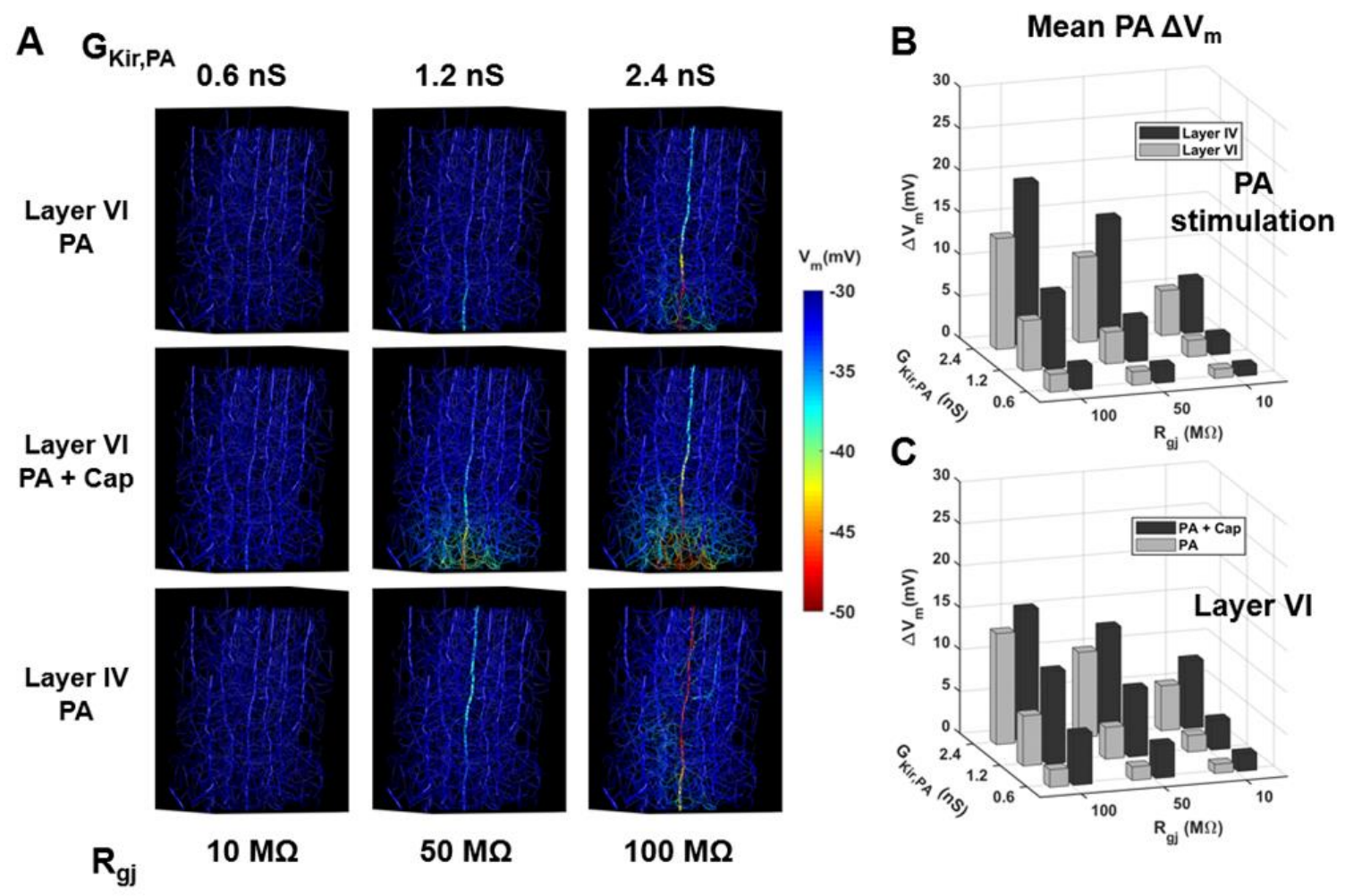

Figure 2-6 Parenchymal arteriole vs. Capillary stimulation in vivo. A 1000x500x500 $\mu \mathrm{m} 3$ network, reconstructed from [96], containing approximately $3000 \mathrm{cECs}$, feeding PAs and draining PVs is utilized to show the effect of capillary vs parenchymal arteriole potassium stimulation on the propagation of the vasodilatory signal along the feeding PA, towards the surface arteries. Each cEC is modeled using Model A. (A) Color coded figures show the level of hyperpolarization reached to the PA as a function of GKir,PA, Rgj in capillaries, location of stimulus, and co-stimulation of PA with the surrounding capillaries in response to a localized potassium challenge $(10 \mathrm{mM}[\mathrm{K}+] \mathrm{o})$. (B) Summary plots comparing the average level of PA hyperpolarization when a $100 \mu \mathrm{m}$ of PAs is stimulated in layer IV vs layer VI, as a function of GKir,PA and Rgj. (C) Summary plots comparing the average level of PA hyperpolarization when in addition to the PA, the surrounding $100 \mu \mathrm{m} 3$ capillary region in layer VI is also stimulated with increased extracellular potassium. In all simulation performed in this figure, $c E C$ GKir $=0.3 \mathrm{nS}$ and Rgj,PA $=10 \mathrm{M} \Omega$. PVs are assumed to have the same Gbg and Rgj as PAs, but lack Kir channels. The rest of the parameters used in this simulation is summarized in Table 2-1.

Conduction through capillaries may provide an alternative/parallel pathway for communicating neuronal activity to the vasculature and elicit retrograde electrical signaling in the brain. Sufficient cEC Kir activity $\left(\mathrm{G}_{\mathrm{Kir}}=1.2 \mathrm{nS}\right)$ can promote regenerative propagation of electrical signals though the capillary network (Figure 2-7) and upstream to the PAs and PVs. In this representative simulation, cECs are stimulated with $10 \mathrm{mM} \mathrm{K}^{+}$and resulting local hyperpolarization ascends through the capillary network at a speed of 0.2 $\mathrm{mm} / \mathrm{s}$ (Figure 2-7). The relative slow velocity of electrical propagation reflects the weak 
cEC coupling $\left(\mathrm{R}_{\mathrm{gj}}=500 \mathrm{M} \Omega\right)$ assumed in the simulation. cEC hyperpolarization disperses and hyperpolarizes nearby PAs and surface arteries to increase local CBF. Simulations in Figure 2-7 also indicate that when a region with high level of $\mathrm{cEC} \mathrm{G}_{\mathrm{Kir}}$ is surrounded by capillaries with lower $\mathrm{G}_{\mathrm{Kir}}(0.3 \mathrm{nS})$, the conducted hyperpolarization along the microvasculature can be spatially confined (Figure 2-7 top view). Thus, model predicts that spatially-confined electrical signals may ascend through capillaries, with conduction velocities that can differ based on the level of cEC coupling (propagation velocity is inversely proportional to $\mathrm{R}_{\mathrm{g}}$ ).

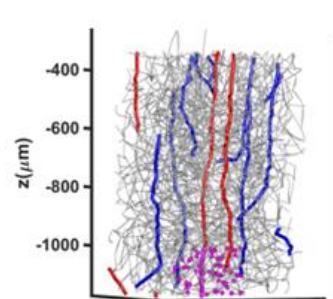

2.50 seconds

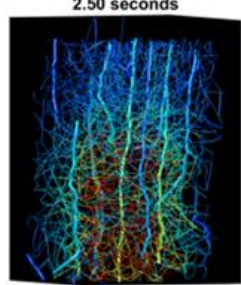

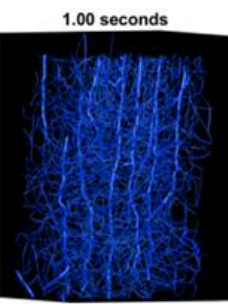

3.00 seconds

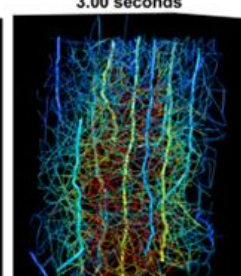

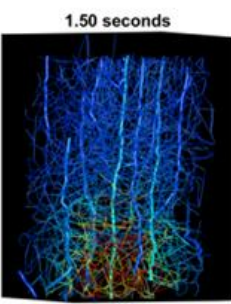

3.50 seconds

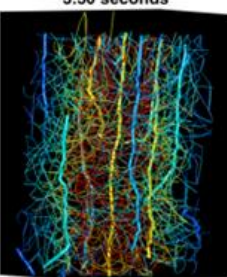

2.00 seconds

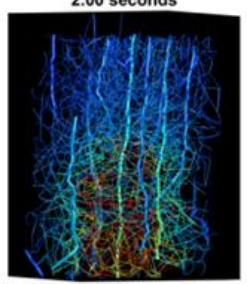

4.00 seconds

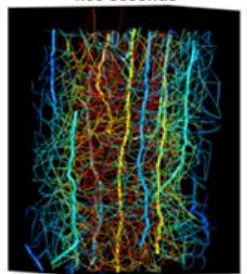

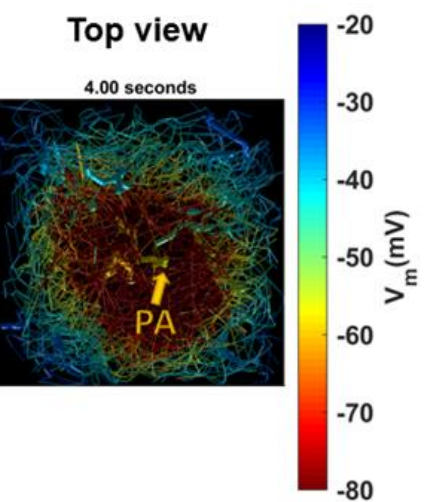

Figure 2-7 Retrograde vasodilatory signaling from capillaries to PAs in vivo. The same network as the one used in Figure 2-6 is utilized to identify requirements of the system to conduct a potassium-induced capillaryinitiated vasodilatory signal from deep layers of the cortex towards the surface arteries. Each cEC is modeled using Model A. A $100 \mu \mathrm{m}^{3}$ of cECs (highlighted in purple in the top left panel) is stimulated with $10 \mathrm{mM}$ of $\mathrm{K}^{+}$. Simulations are performed for 4 seconds with $\mathrm{R}_{\mathrm{gj}}=500 \mathrm{M} \Omega$ (potassium stimulation onset is at one second into the simulation). The Kir density is increased in a region surrounding the PA $\left(\mathrm{G}_{\mathrm{Kir}}=1.2 \mathrm{nS}\right)$, and the rest of the cECs have the control $\mathrm{G}_{\mathrm{Kir}}=0.3 \mathrm{nS}$. The progression of the hyperpolarizing wavefront is shown with increments of $0.5 \mathrm{~s}$ in color coded panels, where the retrograde vasodilatory signal is confined in the "sensitized" region. The rightmost panel shows the top view of the network after 4 seconds, where the hyperpolarizing front has reached close to the surface vasculature. Significant levels of hyperpolarization can be observed in the PA as the hyperpolarizing wave propagates upstream towards the surface vessels. $\mathrm{R}_{\mathrm{g}, \mathrm{PA}}=$ $10 \mathrm{M} \Omega$. PVs are assumed to have the same $\mathrm{G}_{\mathrm{bg}}$ and $\mathrm{R}_{\mathrm{gj}}$ as PAs, but lack Kir channels. The rest of parameters are as stated in Table 2-1. 


\subsection{DISCUSSION}

cECs in the brain have been extensively studied in the context of the blood-brain barrier; yet, very little is known about their electrophysiological properties. The high capillary density in the brain allows for a close proximity of cECs to every neuron; thus, uniquely positions them to probe neuronal activity. However, whether the capillary endothelium is capable of sensing neuronal activity and transmitting signals to cause upstream vasodilation — a question with profound implications for global NVC mechanisms-has received little research attention. Recent experimental evidence suggest that capillaries may act as a sensory web to detect neuronal activity through Kir channel-mediated sensing of local elevations in $\left[\mathrm{K}^{+}\right]_{\mathrm{o}}$. These changes can be communicated by a hyperpolarizing electrical signal from capillaries to PAs through the endothelial layer. Together with direct activation of arteriolar (SMC and EC) Kir channels by extracellular $\mathrm{K}^{+}$from astrocytes and/or neurons, the capillary-initiated electrical signals can produce stable vasodilation and hyperemia. Model analysis, in this study, elucidates the biophysical determinants of $\mathrm{K}^{+}$ sensing by cECs and the parameters/mechanisms that regulate retrograde electrical signal transmission from capillaries to upstream feeding arteries in the brain.

\subsubsection{Kir2.1 channel: an exquisite sensor of $\mathrm{K}^{+}$}

Activation of Kir2.1 family members by $\mathrm{V}_{\mathrm{m}}$ and $\left[\mathrm{K}^{+}\right]_{\mathrm{o}}$ has been well characterized, and likely reflects unblock of the channels by internal polyamines [106-108]. As $\left[\mathrm{K}^{+}\right]_{\mathrm{o}}$ increases, the current-voltage relationship shifts in an upward and rightward direction (Figure 2-2 B-D) due to an increase in the $\mathrm{G}_{\mathrm{Kir}}, \mathrm{E}_{\mathrm{K}}$, and $\mathrm{V}_{0.5}$ (Equation 2-2). Consequently, the Kir activity at resting $\mathrm{V}_{\mathrm{m}}$ increases. For example, at resting conditions $\left(\mathrm{V}_{\mathrm{m}} \approx-30 \mathrm{mV}\right)$, 
increasing $\left[\mathrm{K}^{+}\right]_{\mathrm{o}}$ from $3 \mathrm{mM}$ to $10 \mathrm{mM}$, causes an approximately 100 -fold increase in Kir chord conductance (from $\sim 0.0006 \mathrm{nS}$ to $\sim 0.07 \mathrm{nS}$ at $-30 \mathrm{mV}$ ). This value is almost 10 times lower than the maximum chord conductance at $10 \mathrm{mM}(\sim 0.6 \mathrm{nS})$, which yields to a small (sub-picoampere) increase in the outward current. The $\mathrm{K}^{+}$-induced Kir opening, however, can be self-amplifying since the cell hyperpolarizes in response to the increased $\mathrm{I}_{\mathrm{Kir}}$, which can further increase activity due to the channel's negative slope conductance. Mathematical analysis (Figure 2-2) quantifies this effect and shows how small increases in $\left[\mathrm{K}^{+}\right]_{\mathrm{o}}$ can exert a powerful hyperpolarizing effect as a result of a bifurcation that promotes a hyperpolarizing jump when a threshold $\left[\mathrm{K}^{+}\right]_{\mathrm{o}}$ is crossed. Thus, Kir channel acts essentially as an "on-off" switch to rapidly and profoundly hyperpolarize the cell membrane in capillaries and arterioles when extracellular $\mathrm{K}^{+}$increases.

\subsubsection{Kir-mediated control of microvascular $V_{m}$}

ECs are classified as electrically non-excitable cells; however, studies have often revealed non-typical $\mathrm{V}_{\mathrm{m}}$ dynamics: Two resting potentials have been observed in cultured EC monolayers [109, 110], and isolated ECs [111] may exhibit spontaneous transitions between a hyperpolarized and a depolarized potential when bath $\mathrm{K}^{+}$increases [111-113]. Similarly, all or none responses to increasing $\left[\mathrm{K}^{+}\right]_{\mathrm{o}}[87]$ and spontaneous transitions between two polarization levels ([102] and Fabrice Debartrand, unpublished data) have been documented in isolated PAs, and evidence for $\mathrm{V}_{\mathrm{m}}$ bistability in cerebral SMCs and arterioles have also been presented [90]. Although the presence of Kir current is a prerequisite for these responses, the underlying mechanisms and physiological relevance have not been elucidated. Model simulations performed in this study show how bistable 
cells can amplify incoming electrical signals and under certain conditions promote excitability and regenerative conduction (Figure 2-3 F,Figure 2-5 to Figure 2-7).

\subsubsection{Are ECs capable of carrying APs?}

The inward rectification of the Kir by channel pore blockers provide the characteristic Nshaped current-voltage relationship (Figure 2-2 B) that resembles, albeit inverted, the $\mathrm{V}_{\mathrm{m}}$ dependence of the $\mathrm{Na}_{\mathrm{v}}$ activation gate in neuronal APs (Appendix D2). The nonlinear dependence on voltage allows for the creation of bistable systems in capillaries and neurons that can promote hyperpolarizing and depolarizing jumps, respectively, upon threshold excitation (Figure 2-2 and Appendix D2). Model simulations demonstrate that AP-like electrical responses are possible in capillaries when there is sufficient Kir activity to make the majority of cECs bistable (Figure 2-3). Experiments have provided evidence of bistability in different types of ECs [87] and an elaborate machinery exist to modulate Kir activity (i.e. regulation by $\left.\left[\mathrm{K}^{+}\right]_{\mathrm{o}}, \mathrm{PIP}_{2} / \mathrm{ATP}\right)[45,46]$ and thus adjust/regulate the percentage of bistable cECs. Compared to neurons, capillaries form a low intensity system with transmembrane current densities in the order of $0.1 \mathrm{pA} / \mathrm{pF}$, almost 1000 -fold lower that typical current densities in mammalian axons [114]. Higher membrane $\left(\mathrm{r}_{\mathrm{m}}=30 \mathrm{M} \Omega \mathrm{cm}\right)$ and axial $\left(r_{i}=5-500 G \Omega / c m\right.$ for $\left.R_{g j}=10 M \Omega-1 G \Omega\right)$ resistances yield much lower conduction velocities (under passive or regenerative conditions) in capillaries ( $\mathrm{mm} / \mathrm{s}$ ) compared to neurons (m/s) (Figure 2-3).

\subsubsection{Evidence for Kir-mediated amplification of electrical signaling}

$\mathrm{K}^{+}$stimulation of a few cECs can yield robust Kir-mediated hyperpolarization and dilation of the feeding PA several hundred microns away in an ex vivo preparation and in vivo [30]. 
The levels of evoked hyperpolarization are remarkable when one considers the small outward Kir currents recorded in isolated cECs [30] and the small number of cECs stimulated in the experiment in Figure 2-4 A. Model analysis consolidates experimental data in isolated cECs with integrated responses in capillary networks and shows that the evoked arteriolar hyperpolarization cannot be explained by a passive electrical spread from the stimulated capillary segment to the PA (Figure 2-4 B,C). We thus, propose that the significant arteriolar hyperpolarization in the ex vivo Cap-PA preparation and in in vivo data [30], suggests amplification of electrical signals in the cerebral microcirculation. Simulations reveal a significant potential of Kir channels for amplifying conducted electrical signals, thus providing the means to induce significant upstream dilations in response to small local stimulatory currents (Figure 2-4 to Figure 2-7).

\subsubsection{Analog versus digital mode of microvascular network hyperpolarization}

Simulations show that progressive increase in the Kir activity can transition a capillary segment (Figure 2-3) or a microcirculatory region (Figure 2-5) from insensitive to $\mathrm{K}^{+}$ stimuli (passive system) to one of intermediate sensitivity with responses graded to the level of stimulation (facilitated conduction), and to a sensitive and binary (on/off) sensory web (excitable with/without propagating front). Thus, dynamic modulation of Kir by $\left[\mathrm{K}^{+}\right]_{\mathrm{o}}$ or $\mathrm{PIP}_{2}$ can regulate system's fidelity/sensitivity and the ability to work in a digital or analog fashion. Binary responses in capillary regions can be integrated to hyperpolarize feeding arteries at levels that increase as more regions are stimulated. Under this scenario the blood flow response can be graded by the number of activated regions (Appendix F2). 


\subsubsection{Excitability and AP-like propagation}

Parametric analysis in models depicting capillary segments or networks revealed conditions that can promote hyperpolarizing jumps upon stimulation and excitability, spatially-confined, without the presence of a propagating hyperpolarizing front. Conditions that promote excitability were identified in short, well-coupled capillary segments after local stimulation (Figure 2-3 F), and in isolated networks (Figure 2-5 A) and larger vascular architectures (Appendix F2) when a small percentage of distributed cECs were stimulated. Experimental data provide evidence for capillary excitability in isolated ex vivo microvascular networks stimulated locally by $10 \mathrm{mM} \mathrm{K}^{+}$(Figure 2-4). Model shows how these hyperpolarizing jumps can be achieved with lower Kir densities than what is required for regenerative conduction and how they could reset following stimulus termination by basal levels of TRPV4 and NaK pump activity (Figure 2-3 C).

Higher Kir density can promote regenerative conduction of electrical signal and translate sub-pico or pico amperes of stimulatory signal to robust dilatory responses several hundred microns away. This condition would provide maximum sensitivity to the capillary network and the ability to modulate flow based on requirements almost at the single neuron level, as local stimulation of few cECs can affect feeding PA $\mathrm{V}_{\mathrm{m}}$. Capillary network operation under this mode, requires high $\mathrm{G}_{\mathrm{Kir}}$ and an active mechanism for network resetting following stimulus termination. Furthermore, as the electrical signal can travel without attenuation in the highly interconnected brain capillary network, a mechanism should exist for spatial confinement. In representative model simulations, robust resetting could be achieved by dynamic downregulation of Kir and/or upregulation of TRPV4 activity 
following the hyperpolarizing jump (Figure 2-4 E). Additionally, the propagating hyperpolarization front could be restricted to "sensitized" capillaries/arterioles with high Kir activity when surrounded by vascular segments with non-regenerative characteristics (i.e. lower GKir) (Figure 2-7). Thus, excitability with (Figure 2-7) or without (Appendix F2) an AP-like propagation can be spatially confined in the capillary network, and an on/off regulation of electrical activity in microscale volumes is predicated when there is sufficient Kir density in the network.

\subsubsection{PA vs capillary mediated retrograde electrical signaling}

Experimental evidence suggests an important role of conduction in functional hyperemia [19]. Although propagating hyperpolarization and vasodilation along the penetrating and surface arteries have attracted the initial focus of investigations, recent evidence shows the importance of electrical propagation along the capillary network [30, 37, 45, 46]. Simulations using realistic representation of the microvascular geometry provide a way to test the physiological relevance of conduction in PAs and capillaries under different scenarios.

Model simulation performed in this study suggest that conduction along PAs can be significantly inhibited by current losses to branching capillaries, particularly in the deeper layers of the cortex where the PAs are surrounded by a dense capillary network (i.e. layer VI vs layer IV of the cortex) (Figure 2-6 B). Model predicts that a similar level of electrical coupling between ECs in the capillaries as in the PAs (i.e. $\mathrm{R}_{\mathrm{gj}}=\mathrm{R}_{\mathrm{g}, \mathrm{PA}}=10 \mathrm{M} \Omega$ ), can have a detrimental effect on electrical signal propagation along the PAs (Figure 2-6 B). For weakly coupled cECs, however, stimulation of a PA and the resulting local 
hyperpolarization can reach the surface microcirculation. Model simulations also predicted conditions where the retrograde signaling can ascend through the capillary network rather than the PA (Figure 2-7). A localized stimulation of the capillary network deep in the cortex can ascend and promote significant hyperpolarization of the surface microcirculation when sufficient Kir density is present and can be spatially confined if capillaries are bounded by a non-excitable region. 
Table 2-1- Model parameters

\begin{tabular}{|c|c|c|c|c|}
\hline Parameter & Description & Control value & Range & Reference \\
\hline \multicolumn{5}{|c|}{ Capillary } \\
\hline$\overline{\mathbf{G}}_{\mathbf{K i r}}$ & Kir constant & $0.18 \pm 0.1\left(\mathrm{nS} / \mathrm{mM}^{0.5}\right)$ & $\begin{array}{l}{[0.05-0.7]} \\
\left(\mathrm{nS} / \mathrm{mM}^{0.5}\right)\end{array}$ & {$[30,45,46]$} \\
\hline GKir & Maximum Kir conductace & $0.3 \pm 0.2(\mathrm{nS})$ & {$[0.1-1.2](\mathrm{nS})$} & $\overline{\mathrm{G}}_{\mathrm{Kir}} \sqrt{\left[\mathrm{K}^{+}\right]_{\mathrm{o}}}$ \\
\hline $\mathbf{V}_{0.5}$ & $\begin{array}{l}\text { Voltage at half maximal } \\
\text { inactivation of Kir current }\end{array}$ & $\mathrm{E}_{\mathrm{K}}+25(\mathrm{mV})$ & $\mathrm{E}_{\mathrm{K}}-\mathrm{E}_{\mathrm{K}}+40(\mathrm{mV})$ & {$[36,89,90]$} \\
\hline $\mathbf{K}$ & Slope factor of Kir current & $7(\mathrm{mV})$ & & [89] \\
\hline $\mathbf{R}_{\mathbf{g j}}$ & Gap junctional resistance & $10(\mathrm{M} \Omega)$ & $10(\mathrm{M} \Omega)-1(\mathrm{G} \Omega)$ & Model Estimate \\
\hline LEC & Length of capillary EC & 20 (um) & & {$[30]$} \\
\hline $\mathbf{r}_{\mathbf{m}}$ & $\begin{array}{l}\text { Membrane resistivity per } \\
\text { unit length }\end{array}$ & $333 \mathrm{G} \Omega \mu \mathrm{m}$ & & $\mathrm{L}_{\mathrm{EC}} / \mathrm{G}_{\mathrm{bg}}$ \\
\hline $\mathbf{r}_{\mathbf{i}}$ & $\begin{array}{l}\text { Axial resistivity per unit } \\
\text { length }\end{array}$ & $0.5(\mathrm{M} \Omega / \mu \mathrm{m})$ & & $\mathrm{R}_{\mathrm{gj}} / \mathrm{L}_{\mathrm{EC}}$ \\
\hline $\mathbf{C}_{\mathbf{m}}$ & Membrane capacitance & $8 \mathrm{pF}$ & & {$[30,45,46]$} \\
\hline \multicolumn{5}{|c|}{ Parenchymal Arteriole } \\
\hline $\mathbf{R}_{\mathbf{m}, \mathbf{E C}}$ & EC membrane resistance & $12 \mathrm{G} \Omega$ & & {$[94,95]$} \\
\hline $\mathbf{R}_{\mathbf{m}, \mathrm{SMC}}$ & SMC membrane resistance & $6 \mathrm{G} \Omega$ & & {$[36,97]$} \\
\hline $\mathbf{R}_{\mathbf{M E}}$ & $\begin{array}{l}\text { Myoendothelial junction } \\
\text { resistance }\end{array}$ & $1 \mathrm{G} \Omega$ & & [97] \\
\hline $\mathbf{R}_{\mathbf{g j}, \mathbf{P A}}$ & $\begin{array}{l}\text { PA gap junctional } \\
\text { resistance }\end{array}$ & $10(\mathrm{M} \Omega)$ & $\begin{array}{c}1(\mathrm{M} \Omega)-50 \\
(\mathrm{M} \Omega)\end{array}$ & \\
\hline Gbg,EC-SMC & $\begin{array}{c}\text { Lumped Background } \\
\text { conductance of an EC- } \\
\text { SMC unit }\end{array}$ & $0.2(\mathrm{nS})$ & $0.1-0.3(\mathrm{nS})$ & $\begin{array}{c}\frac{\left(R_{m, S M C}+R_{M E}+R_{m, 1}\right.}{\left(R_{m, S M C}+R_{M E}\right) R_{m},} \\
{[94]}\end{array}$ \\
\hline GKir,EC-SMC & $\begin{array}{l}\text { Kir conductance of an EC- } \\
\text { SMC unit }\end{array}$ & $0.65(\mathrm{nS})$ & $0.5-1.5(\mathrm{nS})$ & $\begin{array}{c}\mathrm{G}_{\mathrm{Kir}, \mathrm{EC}}+\mathrm{G}_{\mathrm{Kir}, \mathrm{SMC}} \\
{[30,36]} \\
\end{array}$ \\
\hline $\mathbf{V}_{0.5}$ & $\begin{array}{c}\text { Voltage at half maximal } \\
\text { inactivation of Kir current }\end{array}$ & $\mathrm{E}_{\mathrm{K}}+25(\mathrm{mV})$ & $\mathrm{E}_{\mathrm{K}}-\mathrm{E}_{\mathrm{K}}+40(\mathrm{mV})$ & {$[36,89,90]$} \\
\hline $\mathbf{K}$ & Slope factor of Kir current & $7(\mathrm{mV})$ & & [94] \\
\hline ncirc & $\begin{array}{l}\text { Number of ECs in the } \\
\text { circumferential direction }\end{array}$ & $3 \mathrm{ECs}$ & & Model Estimate \\
\hline $\mathbf{r}_{\mathbf{m}, \mathbf{P A}}$ & $\begin{array}{l}\text { PA membrane resistivity } \\
\text { per unit length }\end{array}$ & $55 \mathrm{G} \Omega \mu \mathrm{m}$ & & $\begin{array}{c}\mathrm{L}_{\mathrm{EC}, \mathrm{PA}} /\left(\mathrm{n}_{\mathrm{circ}}\right. \\
\left.\mathrm{G}_{\mathrm{bg}, \mathrm{EC}, \mathrm{SMC}}\right)\end{array}$ \\
\hline $\mathbf{r}_{\mathrm{i}, \mathbf{P A}}$ & $\begin{array}{l}\text { PA axial resistivity per unit } \\
\text { length }\end{array}$ & $0.1(\mathrm{M} \Omega / \mu \mathrm{m})$ & & $\begin{array}{c}\mathrm{R}_{\mathrm{gj}, \mathrm{PA}} /\left(\mathrm{n}_{\mathrm{circ}}\right. \\
\left.\mathrm{L}_{\mathrm{EC}, \mathrm{PA}}\right) \\
\end{array}$ \\
\hline \multicolumn{5}{|c|}{ TRPV4 } \\
\hline P $_{\text {TRPV4,K }}$ & $\begin{array}{c}\text { TRPV4 channel } \\
\text { permeability to } \mathrm{K}^{+} \text {ions }\end{array}$ & $0.5 \times 10-8 \mathrm{~cm} / \mathrm{s}$ & & {$[92]$} \\
\hline PTRPV4,Na & $\begin{array}{c}\text { TRPV4 channel } \\
\text { permeability to } \mathrm{Na}^{+} \text {ions }\end{array}$ & $0.35 \times 10-8 \mathrm{~cm} / \mathrm{s}$ & & {$[92]$} \\
\hline \multicolumn{5}{|c|}{ NaK } \\
\hline $\mathbf{I}_{\text {NaK,max }}$ & $\begin{array}{c}\text { maximum NaK pump } \\
\text { current }\end{array}$ & $29 \mathrm{pA}$ & & [89] \\
\hline $\mathbf{K}_{\mathbf{K}_{\mathbf{o}}}$ & $\begin{array}{l}\text { Half maximal activation of } \\
\mathrm{NaK} \text { pump to }\left[\mathrm{K}^{+}\right]_{\mathrm{o}}\end{array}$ & $4.5 \mathrm{mM}$ & & [115] \\
\hline $\mathbf{m}_{\mathbf{K}_{\mathbf{0}}}$ & {$\left[\mathrm{K}^{+}\right]_{\mathrm{o}}$ slope factor } & $1 \mathrm{mM}$ & & [89] \\
\hline $\mathbf{K}_{\mathrm{Na}_{\mathbf{i}}}$ & $\begin{array}{l}\text { Half maximal activation of } \\
\mathrm{NaK} \text { pump to }\left[\mathrm{Na}^{+}\right]_{\mathrm{i}}\end{array}$ & $14.5 \mathrm{mM}$ & & {$[115]$} \\
\hline $\mathbf{m}_{\mathrm{Na}_{\mathrm{i}}}$ & {$\left[\mathrm{Na}^{+}\right]_{\mathrm{i}}$ slope factor } & $3.5 \mathrm{mM}$ & & Model Estimate \\
\hline
\end{tabular}




\section{APPENDIX A2. VALIDATION OF MINIMAL MODELS OF CEC AGAINST A DETAILED MODEL OF CAPILLARY ELECTROPHYSIOLOGY}
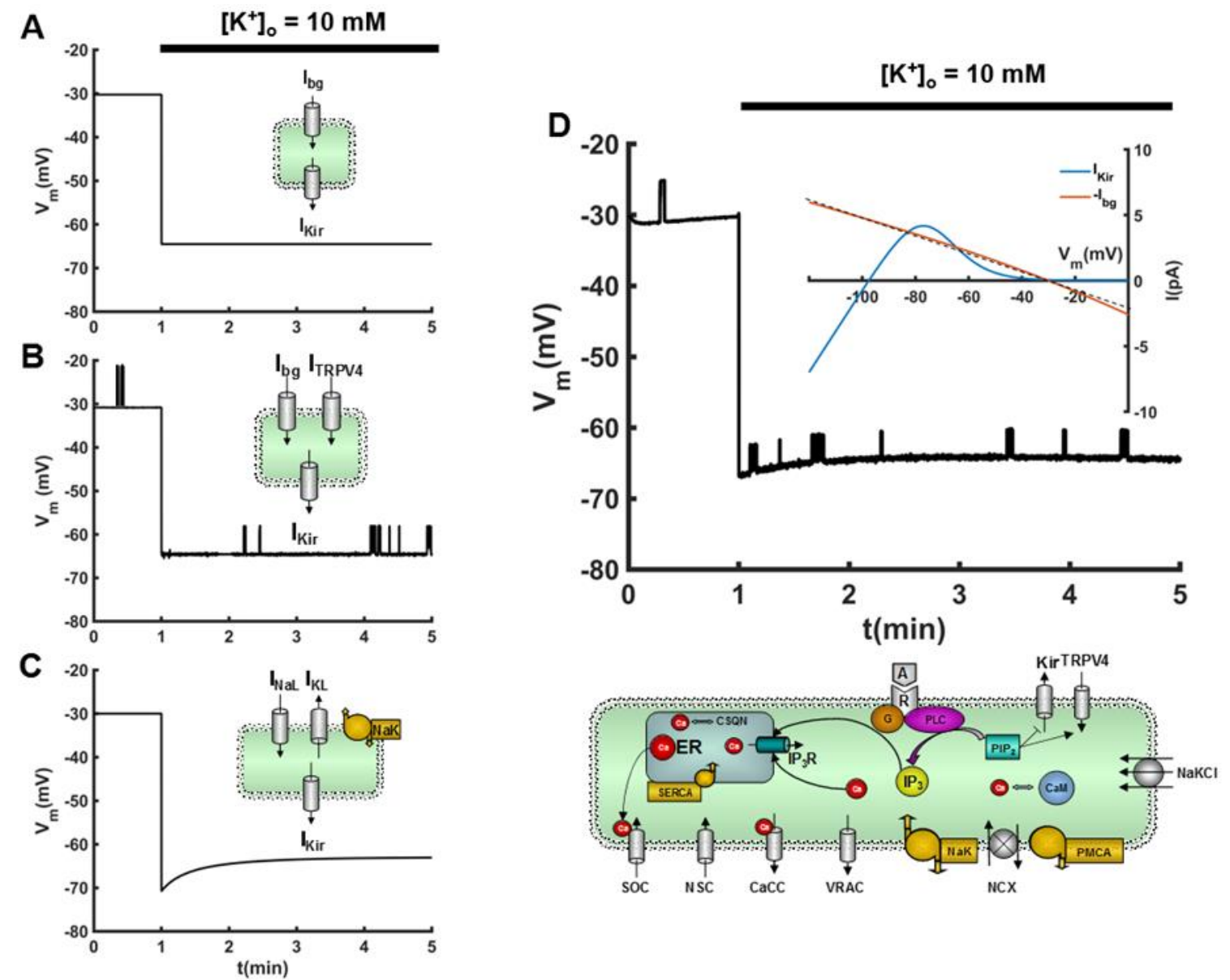

Figure A2- 1 Detailed model of EC electrophysiology [89] was adapted to account for the dynamics of isolated brain cECs (panel D), and to test the simplifying assumptions in the minimal models of cEC in Figure 2-1 A-C. The Kir current is modified to values obtained from patch clamp recordings [30], small and intermediate conductance potassium currents are removed, and the rest of the membrane currents (equivalent to the Ibg in the minimal model of Figure 2-1) are scaled such that the total membrane resistance/conductance matches that of experimental recordings. Stochastic TRPV4 channels are also included in the model with the characteristics of the channel as described in [46] (refer to the Methods section). Panels A-C show the temporal dynamics of $\mathrm{Vm}$ upon potassium stimulation in minimal models of cEC including a Kir current as well as a linear non-specific ohmic background current (model A), a background and TRPV4 current (model $\mathrm{B}$ ), and $\mathrm{NaK}$ and the respective $\mathrm{Na}$ and $\mathrm{K}$ leak currents (model C). The response of the detailed model of cEC to potassium challenge $(10 \mathrm{mM})$ is shown in panel D. The inset shows the traces of the Kir current (blue trace), as well as the summation of the rest of transmembrane currents (denoted as Ibg, orange trace) in the detailed model during a step increase in the $\mathrm{Vm}$ from -120 to $0 \mathrm{mV}$, at $[\mathrm{K}+] \mathrm{o}=3 \mathrm{mM}$ under a voltage clamp condition. As can be observed, lumped transmembrane currents in the detailed model (excluding the Kir current) shows almost a linear trend with $\mathrm{Vm}$, which validates the ohmic assumption of the Ibg in the minimal cEC model in Figure 2-1A (notice the linear fit in the dotted black line). The detailed model shows similar response to the potassium stimulus to the combination of the minimal models in A-C, i.e., the transient TRPV4-dependent depolarizations, the transient hyperpolarization due to the increased activity of NaK pump upon start of the potassium stimulus, and the sustained hyperpolarization to potassium challenge as a result of activation of the Kir current. 


\section{APPENDIX B2: BISTABILITY OF CECS}
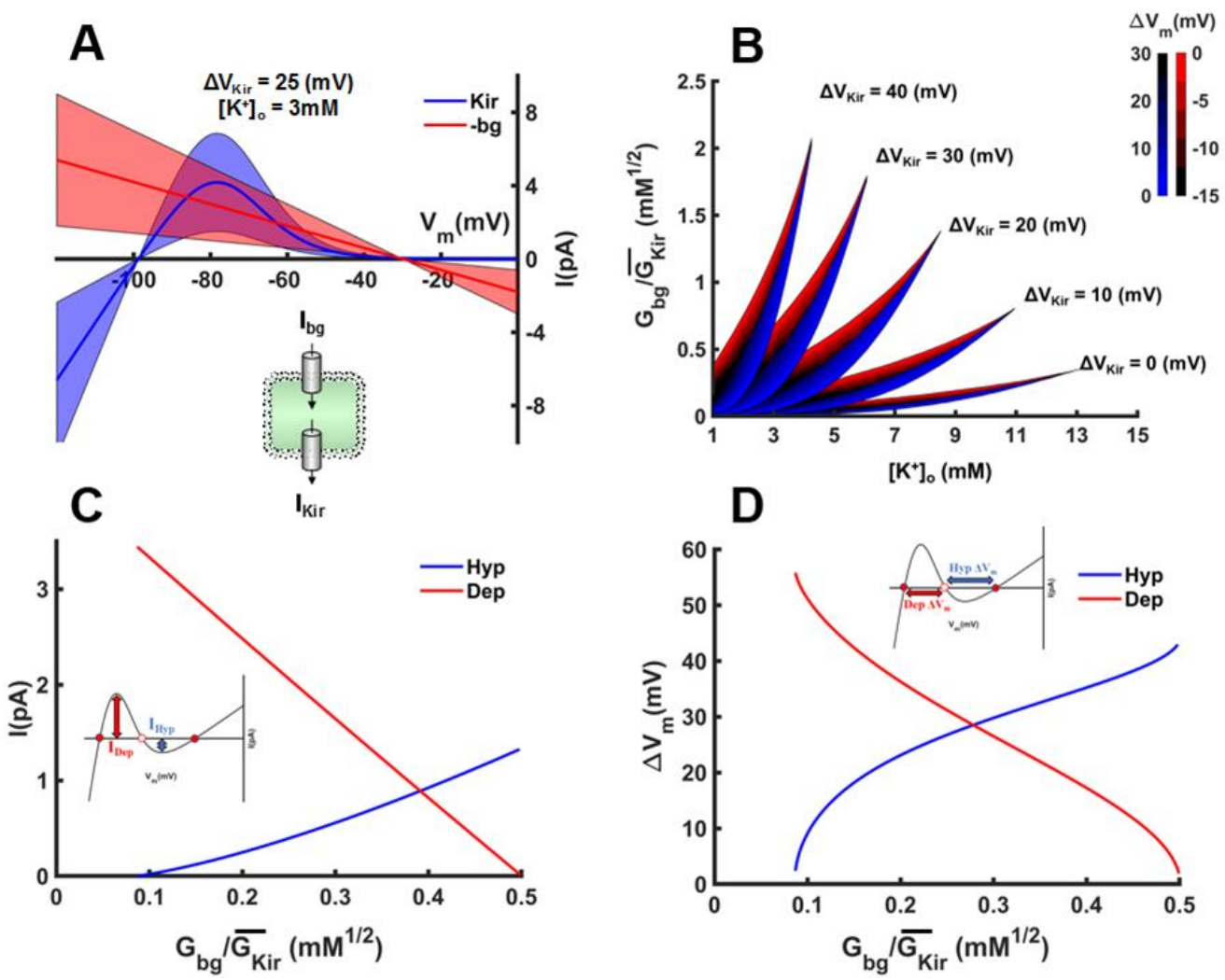

Figure B2- 1 Effect of IKir dependent parameters on the bistability of a single minimal model of cEC (panel A inset) (A) shaded regions show the relative positioning of IKir and Ibg (shown as mean \pm std) of an isolated cEC (inset), based on data from patch clamp recordings of isolated cECs from the mouse brain [30] $\left(\overline{\mathrm{G}}_{\mathrm{Kir}}=0.18+0.1 \mathrm{nS} / \mathrm{mM} 0.5 ; \mathrm{Gbg}=0.06 \pm 0.04(\mathrm{n}=24)\right)$, assuming half maximal inactivation of the Kir current (V0.5) to be $25 \mathrm{mV}$ positive to EK (V0.5 $=\mathrm{EK}+\Delta \mathrm{VKir} ; \Delta \mathrm{VKir}=25 \mathrm{mV})$. Experimental data place cECs mostly within or close to the bistable region; however, according to the graph all four regions of Figure 2-1 E can be possible. (B) Stability diagrams of a single cEC (model A) are depicted with varying values of V0.5 $=\mathrm{EK}+\Delta \mathrm{VKir}$. As V0.5 becomes more depolarized compared to EK (i.e. $\Delta$ VKir becomes more positive), the $[\mathrm{K}+]$ o range at which cEC shows bistability decreases, and the system shows bistable behavior at higher ranges of $\mathrm{G}_{\mathrm{bg}} / \overline{\mathrm{G}}_{\mathrm{Kir}}$. Also, reducing $\Delta \mathrm{VKir}$ shrinks the bistable region, i.e., there is a narrower range of $\mathrm{G}_{\mathrm{bg}} / \overline{\mathrm{G}}_{\mathrm{Kir}}$ where cEC shows bistability in $\mathrm{Vm}$ in response to a given $[\mathrm{Ko}]+$ concentration. Blue regions indicate hyperpolarizing favoring, and red regions indicate depolarizing favoring cECs. Colorbars show the $\mathrm{Vm}$ threshold above which a bistable cEC jumps to hyperpolarized/depolarized potentials during elevation/washout of $[\mathrm{K}+]$ o. (C) The current threshold required for the bistable cEC to jump to hyperpolarized/depolarized potentials as a function of $\mathrm{G}_{\mathrm{bg}} / \overline{\mathrm{G}}_{\mathrm{Kir}}$ at resting potassium concentration ([Ko]+ = $3 \mathrm{mM}$ ). Figure inset shows the total transmembrane current in model A (IKir + Ibg), and circles indicate the steady states of the system where total current is zero. Solid circles indicate stable steady states, and the open circle shows the unstable steady state. The current threshold for regenerative hyperpolarization/depolarization is shown in blue/red, respectively. (D) The voltage threshold required for the bistable cEC to jump to hyperpolarized/depolarized potentials at $[\mathrm{Ko}]+=3 \mathrm{mM}$ as a function of $\mathrm{G}_{\mathrm{bg}} / \overline{\mathrm{G}}_{\mathrm{Kir}}$. The voltage threshold for regenerative hyperpolarization/depolarization are shown in blue/red, respectively, in figure inset. 


\section{APPENDIX C2. TRANSIENT STOCHASTIC TRPV4-MEDIATED DEPOLARIZATIONS IN A DETAILED MODEL OF cEC ELECTROPHYSIOLOGY DURING POTASSIUM CHALLENGE}

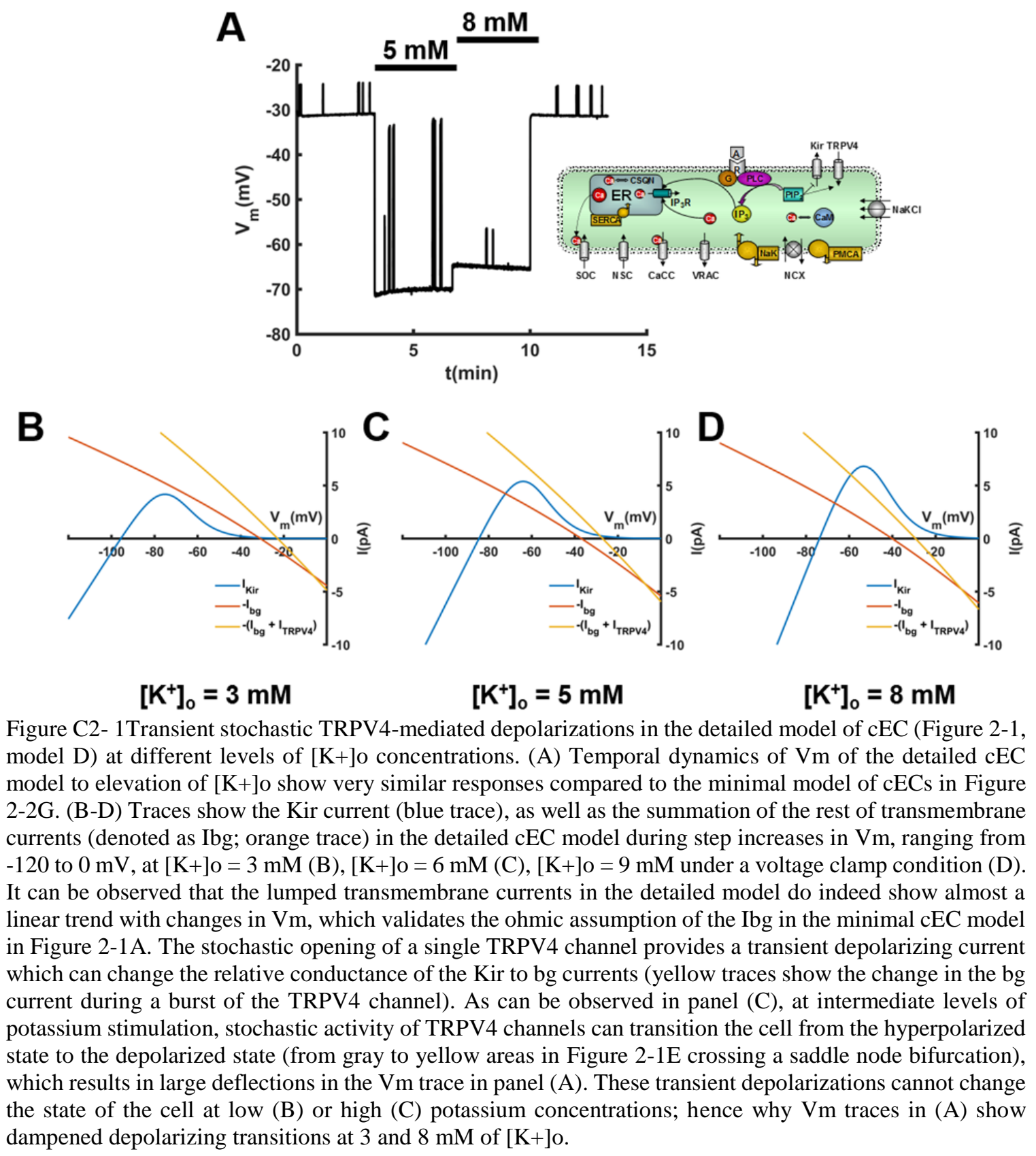




\section{APPENDIX D2. KIR vs NAv MEDIATED BISTABILITY}
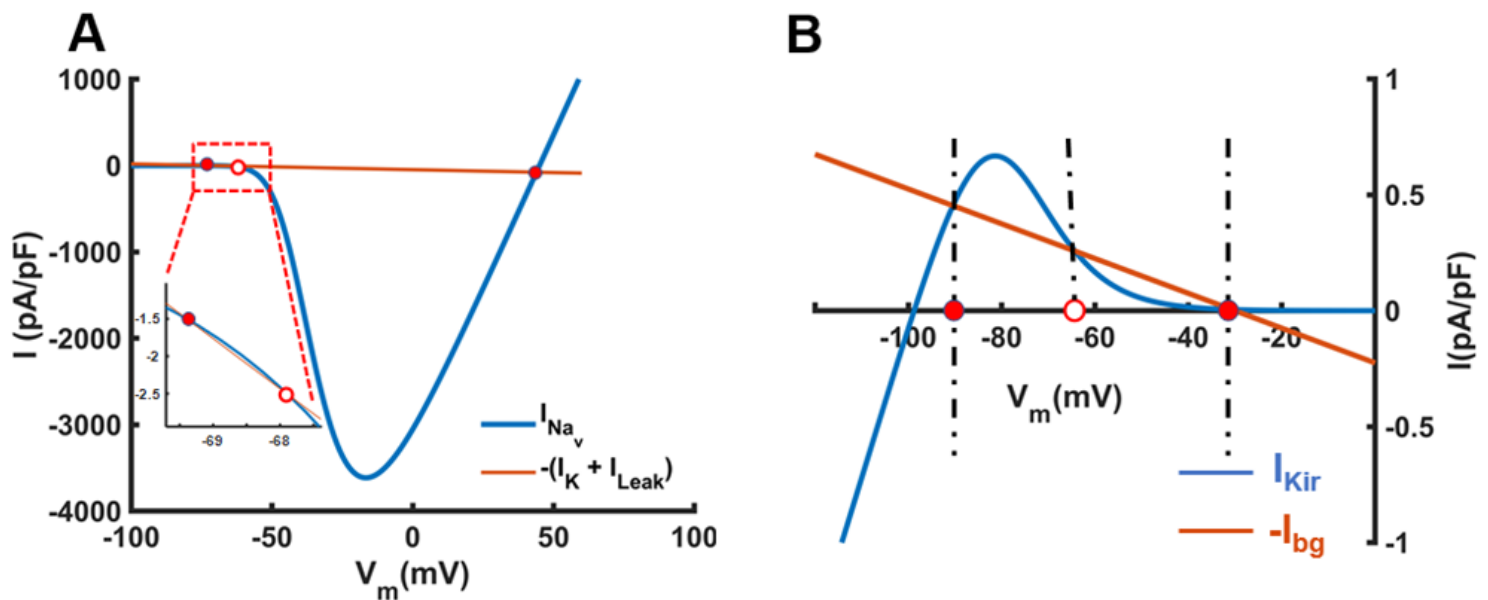

Figure D2- 1 Comparison of Kir-mediated bistability in cECs to $\mathrm{Na}_{\mathrm{v}}$-dependent bistability during an AP in neurons. (A) the steady state voltage gated sodium current [the blue trace: $\mathrm{I}_{\mathrm{Nav}}=\overline{\mathrm{g}}_{\mathrm{Na}} \mathrm{m}_{\mathrm{ss}}^{3} \mathrm{~h}_{0}\left(\mathrm{~V}_{\mathrm{m}}-\mathrm{E}_{\mathrm{Na}}\right)$; where $\overline{\mathrm{g}}_{\mathrm{Na}}$ is the maximum sodium channel conductance, $\mathrm{m}_{\mathrm{ss}}$ is the voltage dependent steady state profile of sodium channel activation gate, $\mathrm{h}_{0}$ is the fraction of open sodium channel inactivation gate at time zero, and $\mathrm{E}_{\mathrm{Na}}$ is the reversal potential of sodium ions] can form a bistable system with the summation of potassium and leak currents [the orange trace: $\mathrm{I}_{\mathrm{K}}=\overline{\mathrm{g}}_{\mathrm{K}} \mathrm{n}_{0}^{4}\left(\mathrm{~V}_{\mathrm{m}}-\mathrm{E}_{\mathrm{K}}\right) ; \mathrm{I}_{\text {Leak }}=\overline{\mathrm{g}}_{\text {Leak }}\left(\mathrm{V}_{\mathrm{m}}-\mathrm{E}_{\text {Leak }}\right)$; equivalent to $\mathrm{I}_{\mathrm{bg}}$ in cEC model $\mathrm{A}$, where $\overline{\mathrm{g}}_{\mathrm{K}}$ and $\overline{\mathrm{g}}_{\text {Leak }}$ are maximum conductance of potassium and leak channels respectively, $\mathrm{n}_{0}$ is the fraction of open potassium channel activation gates at time zero, and $\mathrm{E}_{\mathrm{K}}$ and $\mathrm{E}_{\mathrm{Leak}}$ are the reversal potential of potassium ions and the leak current, respectively], prior to the delayed activation of potassium currents in a typical Hodgkin-Huxely model of an action potential. Solid circles indicate the stable steady states of the neuron, one at hyperpolarized potentials around resting membrane potential of neurons $\approx-70$ $\mathrm{mV}$, and the other at depolarized voltages $\approx 40 \mathrm{mV}$, the maximum level of $\mathrm{V}_{\mathrm{m}}$ during the depolarization phase of an action potential. The open circle indicates the unstable steady state, the threshold $\mathrm{V}_{\mathrm{m}}$ above which an AP can be fired. (B) A bistable cEC represented in model A, shows bistability in the membrane potential attributed to the nonlinear dynamics of the Kir relative to the bg current. Comparison of panels (A) and (B) show similar, albeit inverted, nonlinear dynamics. Also, it is notable that the typical currents for generations of an AP are several orders of magnitude larger than those of $\mathrm{cECs}$, even though the magnitude of $\Delta \mathrm{V}_{\mathrm{m}}$ threshold for the bistable transition is much smaller compared to cECs. 


\section{APPENDIX E2. POTENTIAL RESETTING MECHANISMS AFTER TERMINATION OF K+ STIMULUS IN REGENERATIVE cEC LAYERS.}

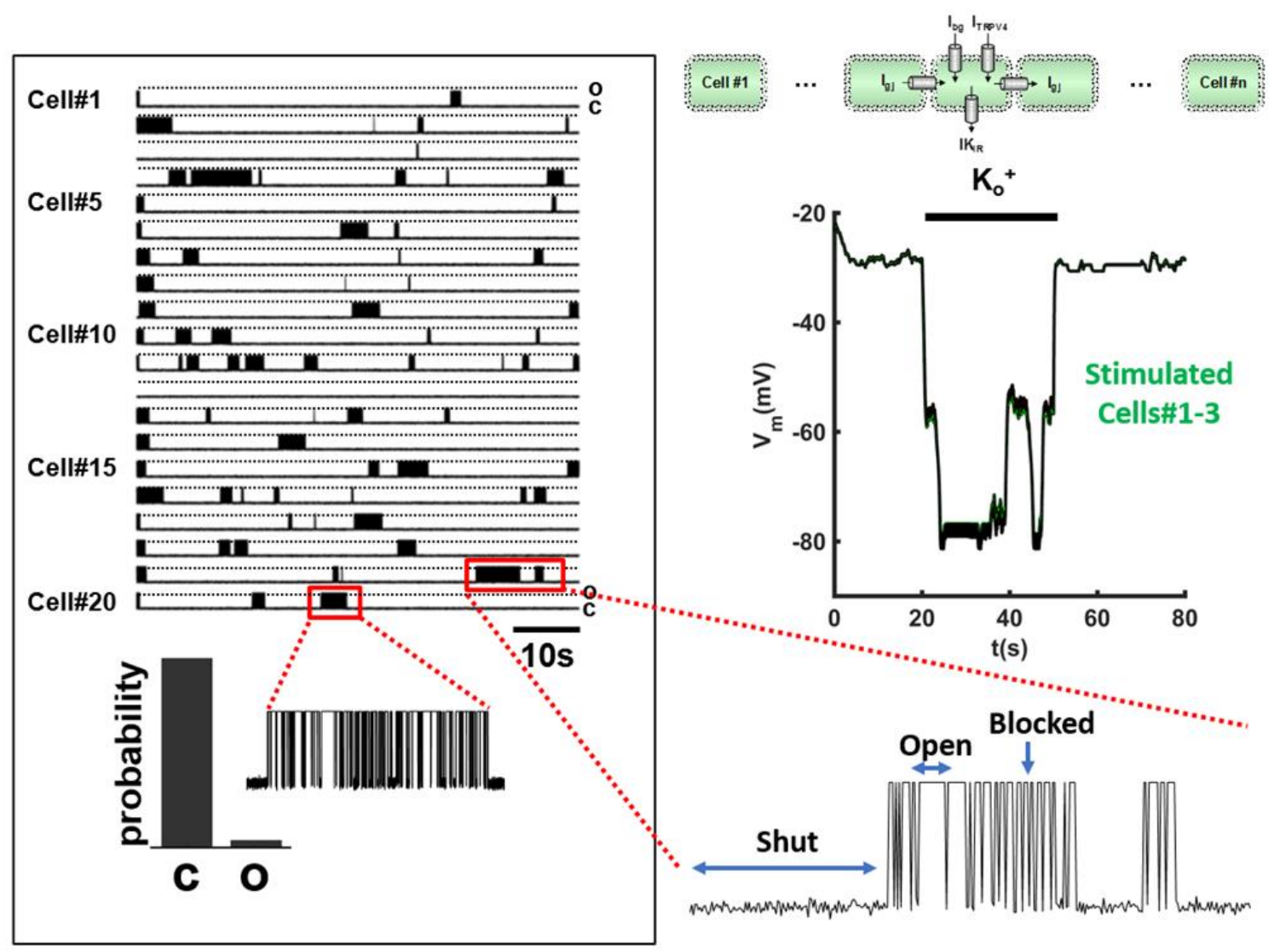

Figure E2- 1 Basal stochastic TRPV4 activity can provide a resetting mechanism after removal of the stimulus in a regenerative layer of connected cECs (Model B). Simulations (temporal Vm traces on the top left) are shown for 20 bistable cECs connected via gap junctions $(\mathrm{Rgj}=10 \mathrm{M} \Omega)$, where the first 3 cells (green traces) from one end of the capillary segment are stimulated by increasing [K+]o from 3 to $10 \mathrm{mM}$. The TRPV4 current in each cEC is modeled using a three-state discrete-time Markov Chain model [91, 92]. The channel can reside in any of the following three states: I) blocked (intraburst short closed state), II) open, and III) shut (interburst long closed state) (Figure inset). The TRPV4 currents (left box) show infrequent single level channel openings with an open probability (time-averaged number of open channels) of $\mathrm{NPo} \approx 0.04$ [46]. In agreement with the experimental data, simulated currents account for one active TRPV4 with bursts of stochastic openings with a mean open time $(90 \mathrm{~ms})$, a mean blocked time (33 ms), a mean shut time (4 $\mathrm{min}$ ), and a mean burst duration ( $20 \mathrm{~s}$ ). Once a sufficient number of spontaneous, stochastic transients of the TRPV4 channels randomly coincide, they provide enough depolarizing current (intermediate jumps in the voltage trace) to reset the system after removal of the stimulus from the hyperpolarized state to resting membrane potentials. 


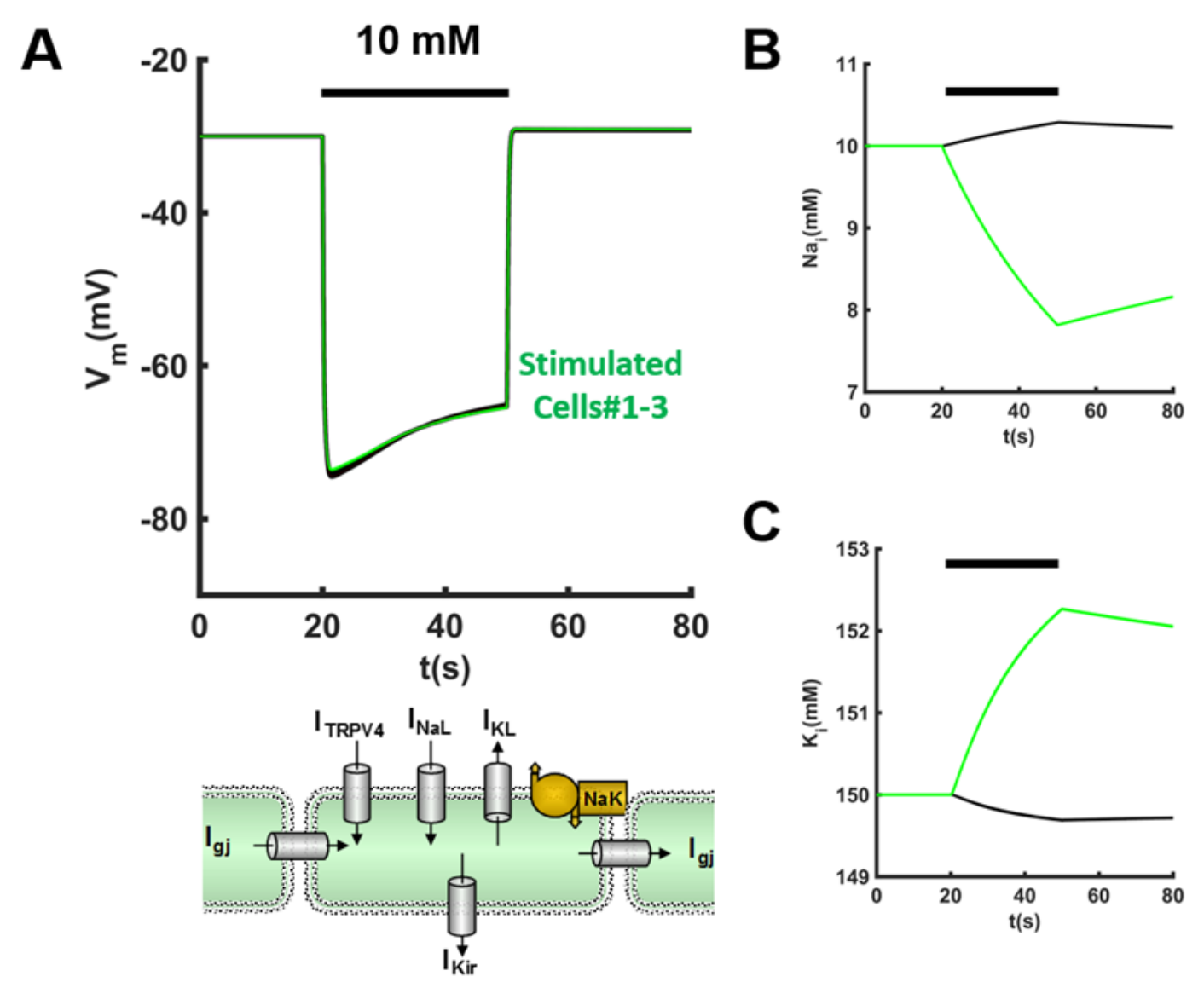

Figure E2- 2NaK pump activity during a $\mathrm{K}+$ challenge in a layer of gap connected cECs can provide initial transient hyperpolarization and a resetting mechanism after the removal of the stimulus. 20 bistable cECs (Model C) are connected via gap junctions $(\operatorname{Rgj}=10 \mathrm{M} \Omega$ ) and the first 3 cells (green traces) from one end are stimulated by increasing [ $\mathrm{K}+$ ] o from 3 to $10 \mathrm{mM}$. The temporal changes of $\mathrm{Vm}$ in the layer is shown in panel (A). Upon the start of the $\mathrm{K}+$ stimulus, the black bar, transient activation of $\mathrm{NaK}$ pump in the stimulated cells results in the elevation of intracellular [K+]i and reduction of intracellular [Na+]i (panels (B) and (C)). The trend is the opposite for unstimulated cells. The transient initial hyperpolarization due to the effect of NaK pump, can help facilitate the regenerative conduction of hyperpolarizing signals even if cECs are not completely in the hyperpolarizing favoring regions of Figure 2-1 E. Consequently, regeneration of the capillary network can happen at lower levels of Kir density, which in turn makes the resetting of the system to resting $\mathrm{Vm}$ values after stimulus termination easier (panel (A)). INaK, $\max =29 \mathrm{pA} ; \mathrm{KKo}=4.5 \mathrm{mM}$; KNai $=14.5 \mathrm{mM} ; \mathrm{mKo}=1 \mathrm{mM} ; \mathrm{mNa}=3.5 \mathrm{mM} ; \bar{G}_{K i r}=0.18 \mathrm{nS} / \mathrm{mM} 0.5$. 

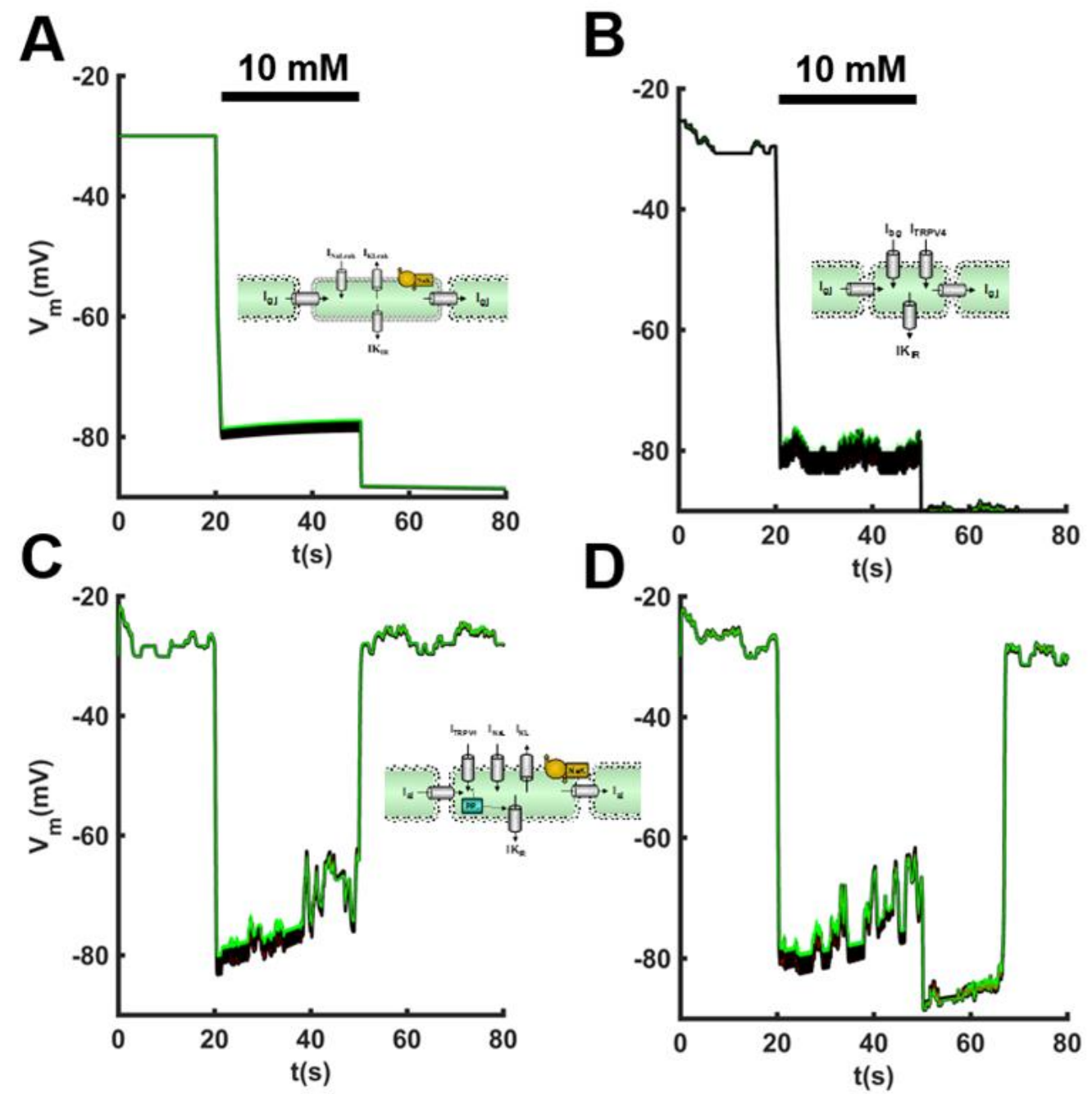

Figure E2- 3Basal TRPV4 and NaK pump activity cannot guarantee resetting the regenerative hyperpolarization in a layer of cECs after the removal of the stimulus. 20 bistable cECs (Models B and C) are connected via gap junctions $(\mathrm{Rgj}=10 \mathrm{M} \Omega)$ and the first 3 cells (green traces) from one end are stimulated by increasing $[\mathrm{K}+]$ o from 3 to $10 \mathrm{mM}$. The temporal changes of $\mathrm{Vm}$ in the layer in panels $\mathrm{A}$ and $\mathrm{B}$ show some example simulations where TRPV4 (A) or NaK (B) activity were not sufficient to reset the system after removal of the potassium stimulation. Parameters are similar to those in Figure E2-2, except for increased $\bar{G}_{\text {Kir }}=0.3 \mathrm{nS} / \mathrm{mM} 0.5$. Regulation of TRPV4 and Kir activity upon prolonged K+ stimulations can provide a more robust resetting mechanism of regenerative hyperpolarization after the removal of the stimulus. The temporal changes of $\mathrm{Vm}$ in the layer in panels $\mathrm{C}$ and $\mathrm{D}$ show that the increase in the activity of TRPV4 and gradual suppression of Kir activity, can provide a robust resetting mechanism for the hyperpolarized cEC layer. Depending on the time constant of PIP2 dependent regulation of Kir/TRPV4 currents, cells can reset instantaneously (C), or with some delays after the removal of $\mathrm{K}+$ stimulus (D). 


\section{APPENDIX F2. Analog versus digital mode of microvascular network hyperpolarization}
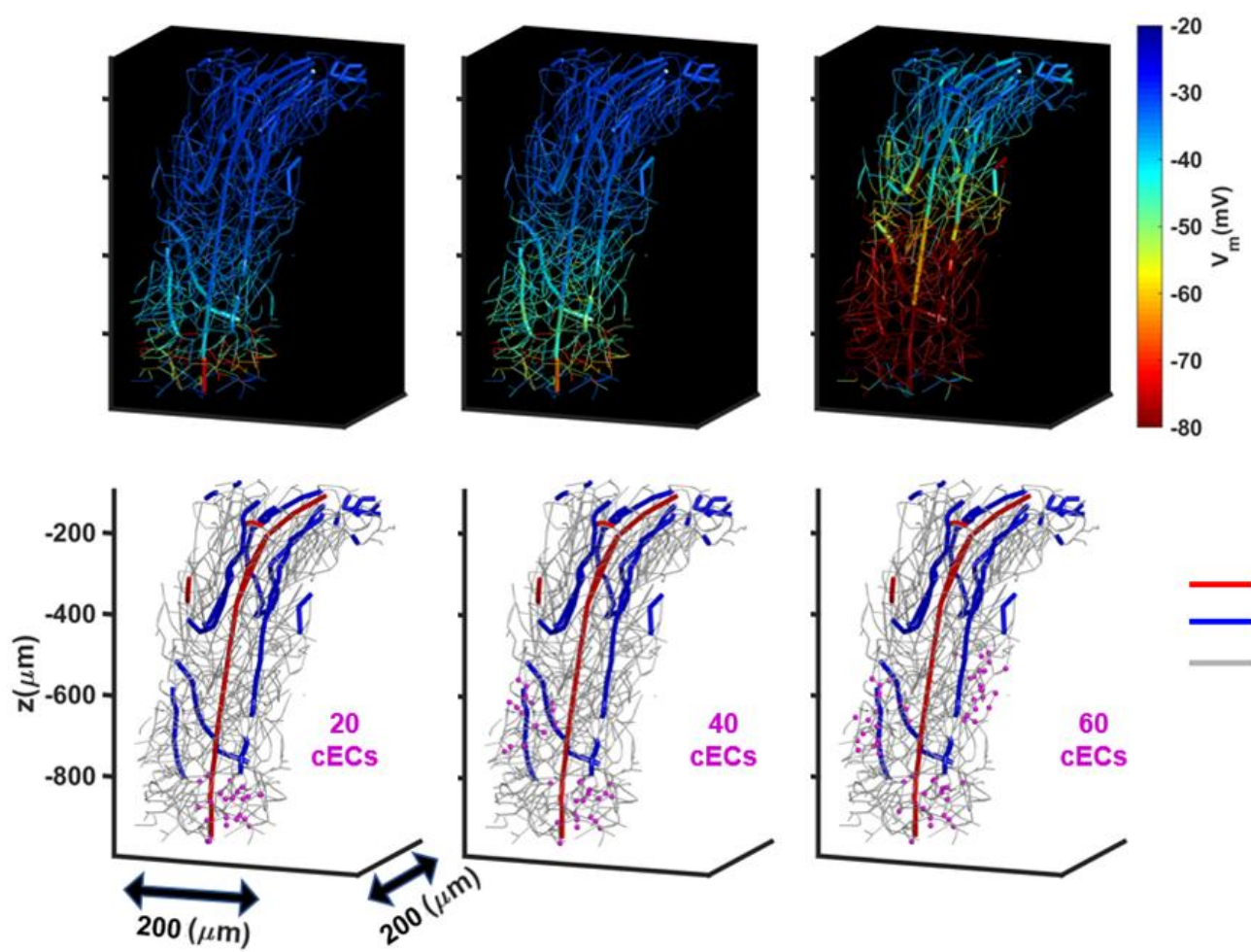

\section{Arterioles Venioles Capillaries}

Figure F2- 1 Integration of binary capillary modules in the hyperpolarization of upstream parenchymal arterioles. Top row shows the predicted Vm hyperpolarization (color coded) in a capillary network reconstruction from the vibrissa primary sensory cortex of mouse (adapted from [96]) containing approximately $1000 \mathrm{cECs}$, in response to stimulation of different regions of cECs $(20,40$, and $60 \mathrm{cECs})$ with $10 \mathrm{mM}$ of $[\mathrm{K}+]$ o. Each cEC is modeled with Model A (Figure 2-1). PAs, parenchymal venules, and capillaries are depicted as red, blue and gray, respectively (bottom panels). Simulations show that at $\mathrm{cEC}$ GKir $=0.5 \mathrm{nS}$ (at $3 \mathrm{mM}$ of $[\mathrm{K}+] \mathrm{o}$ ), stimulation of the capillary regions results in a localized hyperpolarization jump in the capillaries (i.e. binary regions), which can progressively added to increase the transmitted current to the nearby PA (top row from left to right). The parameters used for the simulations of this figure are summarized in Table 2-1. 


\section{CHAPTER 3 DISRUPTION OF NVC IN SMALL VESSEL PATHOLOGIES: A THEORETICAL ANALYSIS}

The content of this chapter (with slight modifications) is published in Microcirculation as Koide M, Moshkforoush A, Tsoukias NM, Hill-Eubanks DC, Wellman GC, Nelson MT, Dabertrand F. "The Yin and Yang of KV Channels in Cerebral Small Vessel Pathologies" [52].

\subsection{INTRODUCTION}

Small vessel disease (SVD) is a group of currently untreated pathologies associated with the dysfunction of small cerebral vessels in brain that have been shown to be directly involved in major neurological conditions including stroke, dementia, and cognitive decline $[7,116]$. Over the past few decades, major progress has been made in determining the underlying molecular mechanisms involved in some members of this family (particularly cerebral autosomal dominant arteriopathy with subcortical infarcts and leukoencephalopathy (CADASIL), and subarachnoid hemorrhage (SAH)), indicating an alteration in the number of functional voltage-gated potassium (Kv) channels in smooth muscle cells (SMCs) of parenchymal arterioles (PAs) [117-123].

$\mathrm{Kv}$ channels form a family of 12 isoforms $(\mathrm{Kv} 1-\mathrm{Kv} 12)$ which share a common structure of an assembly of four pore-forming $\alpha$ subunits and auxiliary $\beta$ subunits $[51,124]$. Under physiological conditions, these channels have a crucial role in determining the myogenic tone of vascular SMCs $[120,125,126]$, and act as the major opposing influence on 
pressure-induced constrictions [127]. The involvement of different subtypes of Kv1 channels, predominantly $\mathrm{Kv} 1.2$ and $\mathrm{Kv} 1.5$, in tone regulation of cerebral vasculature was observed in multiple animal species. These include isolated pressurized parenchymal arterioles and cortical brain slices of the rat [128], small rat cerebral arteries [129], terminal arterioles from rabbit cerebral circulation [130], precapillary arterioles of the murine cerebral circulation [131], and parenchymal arterioles (PAs) of the mice [120]. Similarly, Kv2 currents, primarily Kv2.1 subfamily, and their role in counteracting myogenic constriction in rat and mice cerebral arteries, has been reported in literature [132-134]. Other groups also provided compelling evidence supporting the contribution of different subtypes of Kv7 subfamily in myogenic control of VSMCs in cerebral circulation [135, 136].

Interestingly, the two aforementioned SVD pathologies, i.e. CADASIL and SAH, show opposite alterations in the density of Kv channels in PA SMCs, predominantly Kv1.2 and 1.5 subtypes. In $\mathrm{SAH}$, significant decrease in the number of $\mathrm{Kv}$ channels ( 48\%) is observed, which has resulted in an increased vascular tone and a reduced cerebral blood flow (CBF) [121, 123]. Conversely, CADASIL mouse models showed a marked increase $(\sim 57 \%)$ in the density of $\mathrm{Kv}$ channels and exhibited reduced levels of vasoconstriction $[118,120,125]$. Also, these studies reported that the gating characteristics of Kv channels in PA SMCs were not significantly affected in the diseased animal models, which points to the sole contribution of the $\mathrm{Kv}$ channel density, and not its gating dynamics, on the abnormalities observed in these SVD pathologies. 
Along with $\mathrm{Kv}$ channels, the expression of three other members of $\mathrm{K}^{+}$channels, namely large conductance calcium activated potassium channel (BKCa), inward rectifying potassium (Kir), and ATP-sensitive potassium (KATP), in the arteriolar SMC has been well-documented in literature [127, 137-139]. However, in contrast to SMCs of many other vascular beds, application of $\mathrm{K}_{\mathrm{ATP}}$ agonist cromakalim in PAs, as well as blockade of BKCa channels paxilline did not affect the arteriolar tone under physiological conditions [48, 49, 140, 141]. On the other hand, Kir channels are expressed in both endothelial (EC) and SMCs of PAs [36-38, 142]. Although chemical inhibition, with $\mathrm{Ba}^{2+}$, or genetic ablation of these channels does not affect the resting diameter of PAs [37], small elevations of extracellular $\mathrm{K}^{+}$concentrations $\left(\left[\mathrm{K}^{+}\right]_{\mathrm{o}}\right)$ has caused near maximal dilations in these vessels [37, 127]. As also discussed in CHAPTER 2, this phenomenon is due to the synergistic effect of Kir channels being $\left[\mathrm{K}^{+}\right]_{\mathrm{o}}$ sensors and the self-amplification of their outward current upon hyperpolarization.

Taken together, these observations point to the importance of the interplay between Kv and Kir channels in the regulation of $\mathrm{CBF}$ and its potential contribution in the abnormalities observed during CADASIL and SAH. In this chapter, we use mathematical modeling to understand how the "Yin and Yang" of Kv channels in SAH and CADASIL, i.e., down and up regulation of these channels, affect the regulation of the SMC tone, and in combination with Kir channels the response of the cell to neuronally induced elevations in $\left[\mathrm{K}^{+}\right]_{\mathrm{o}}$ during NVC. 


\subsection{IMPACT OF KV CHANNEL DENSITY ON THE REGULATION OF THE MYOGENIC TONE IN BRAIN PARENCHYMAL ARTERIOLES}

In a normally functioning brain, arteries and arterioles are partially constricted. This preconstricted state of vessels is largely due to the influence of the intravascular pressure, the regulation of which allows the vessel to further constrict or dilate. This response is known is the myogenic tone and is essential in the CBF autoregulation [137, 143]. Here we focus on the role of Kv1 channels (predominantly Kv1.2/1.5 subtypes) in the regulation of PA SMC tone under normal conditions (control (CTL)), and in the presence of CADASIL and SAH. Figure 3-2 shows the voltage current relationship of Kv channels, fitted using a Boltzmann-type equation, under control and diseased states. As can be observed, the outward $\mathrm{K}^{+}$currents are significantly affected with the increase (CADASIL) and decrease (SAH) in the channel density, relative to CTL conditions. The difference is more pronounced at physiological (Figure inset) and more depolarized range of membrane potentials $\left(\mathrm{V}_{\mathrm{m}}\right)$.

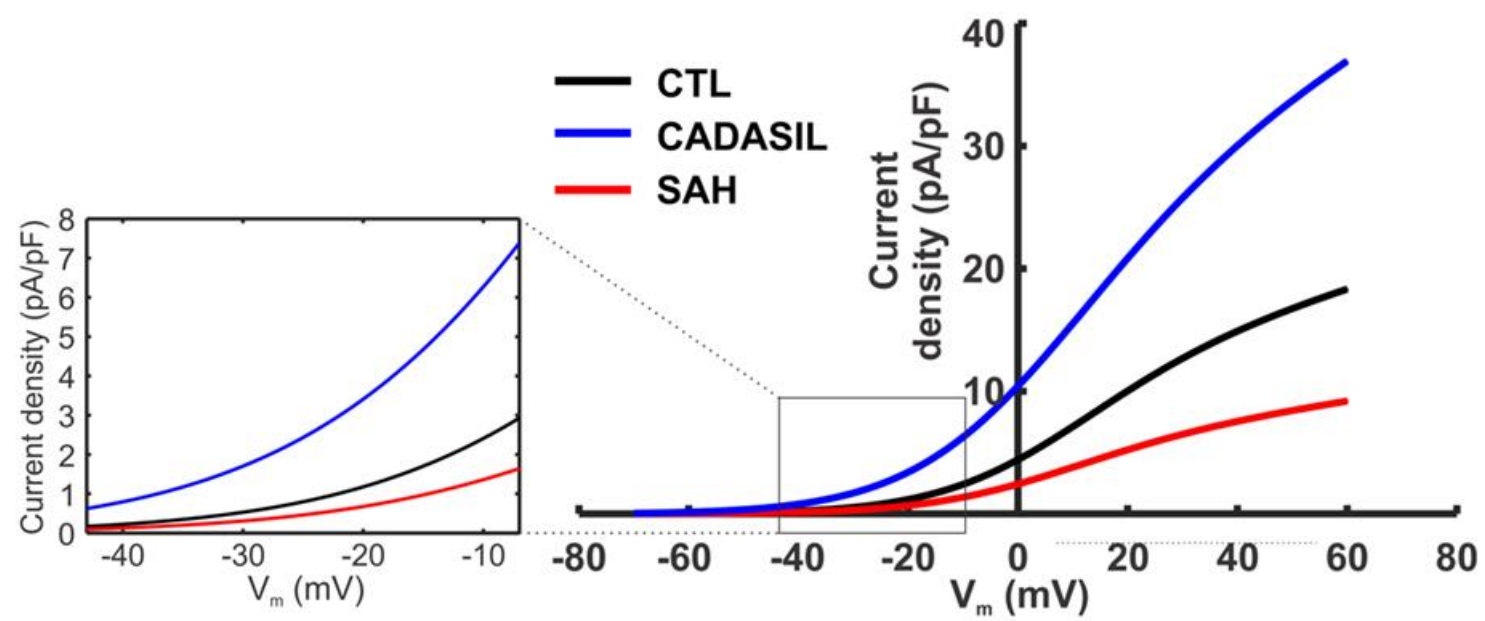

Figure 3-1 KV current density in PA SMCs of normal and diseased animal models at steady state. Current densities are fitted using a linear equation $\mathrm{I}_{\mathrm{Kv}}=\frac{1}{\mathrm{C}_{\mathrm{m}}} \overline{\mathrm{m}}_{\mathrm{Kv}}\left(\mathrm{V}_{\mathrm{m}}-\mathrm{E}_{\mathrm{K}}\right)$ with a Boltzmann-type activation term $\overline{\mathrm{m}}=$ 
$\frac{1}{1+\exp \left(-\left(\frac{v_{m}-v_{K v, 0.5}}{k_{k v}}\right)\right)}$, from experimental data [120] and Koide \& Wellman unpublished data. $C_{m}$ is the membrane capacitance; $\mathrm{V}_{\mathrm{m}}$ is the membrane potential; $\mathrm{G}_{\mathrm{Kv}}$ is the whole-cell conductance of $\mathrm{K}_{\mathrm{V}}$ channels; $\mathrm{E}_{\mathrm{K}}$ is the reversal potential for $\mathrm{K}^{+}$. At physiological membrane potentials pA differences in $\mathrm{K}_{\mathrm{V}}$ currents are predicted (Figure inset). Model parameters: $\left(\mathrm{G}_{\mathrm{kv}}=1.6[\mathrm{nS}] ; \mathrm{V}_{\mathrm{Kv}, 0.5}=6[\mathrm{mV}] ; \mathrm{k}_{\mathrm{kv}}=14[\mathrm{mV}]\right.$ for control; $\mathrm{G}_{\mathrm{kv}}$ $=0.8[\mathrm{nS}] ; \mathrm{V}_{\mathrm{Kv}, 0.5}=6[\mathrm{mV}] ; \mathrm{k}_{\mathrm{kv}}=14[\mathrm{mV}]$ for $\mathrm{SAH} ;$ and $\mathrm{G}_{\mathrm{kv}}=3.2[\mathrm{nS}] ; \mathrm{V}_{\mathrm{Kv}, 0.5}=2.6[\mathrm{mV}] ; \mathrm{k}_{\mathrm{kv}}=15.8[\mathrm{mV}]$ for CADASIL model; $\left.\mathrm{C}_{\mathrm{m}}=12.8[\mathrm{pF}] ;\left[\mathrm{K}^{+}\right]_{\mathrm{i}}=150[\mathrm{mM}] ;\left[\mathrm{K}^{+}\right]_{\mathrm{o}}=3[\mathrm{mM}]\right)$.

The relationship between PA SMCs $\mathrm{V}_{\mathrm{m}}$ and the corresponding myogenic tone for CTL, CADASIL and SAH animal models (taken from experimental recordings in $[120,144]$ ) is shown in Figure 3-2 A. At low intravascular pressure values $(\leq 10 \mathrm{mmHg}), \mathrm{V}_{\mathrm{m}}$ values did not change among the three groups. Under increased pressures $\left(40 \mathrm{mmHg}\right.$ ), however, $\mathrm{V}_{\mathrm{m}}$ was significantly affected, i.e. compared to CTL groups CADASIL models were more hyperpolarized while SAH showed more depolarized membrane potentials. We used a detailed model of a PA SMC (Figure 3-2 B) to investigate whether the observed differences in $\mathrm{V}_{\mathrm{m}}$ among groups can be attributed to the alterations in the $\mathrm{Kv}$ channel density. We adapted the detailed model SMC electrophysiology, originally developed by our group for the dynamics of SMCs in mesenteric arteries [145], by incorporating Kv currents of Figure 3-1, and adjusting the rest of membrane currents such that the $\mathrm{V}_{\mathrm{m}}$ and calcium dynamics match the experimental recordings of $[120,146]$. The model takes into account the important contributors of SMC membrane and intracellular dynamics, including Kv, Kir, $\mathrm{BKCa}$, KATP, voltage-dependent-calcium (VDCC) and non-selective cation (NSC) currents, as well as plasma membrane calcium (PMCA) and $\mathrm{Na}+\mathrm{K}+\mathrm{ATPase}$. The model also accounts for the detailed dynamics of intracellular species and second messengers.

We simulate the increases in intraluminal pressure as the percentage increase in the sodium permeability $\left(\mathrm{P}_{\mathrm{Na}}\right)$ of stretch-activated NSC current on the PA SMC membrane. As can be observed in Figure 3-2 C, at low activation of NSC channels, or equivalently at low 
intraluminal pressures, model simulations predict negligible difference of $\mathrm{V}_{\mathrm{m}}$ and intracellular calcium concentration $\left(\left[\mathrm{Ca}^{2+}\right]_{\mathrm{i}}\right)$ among CTL and diseased (CADASIL and $\mathrm{SAH}$ ) groups, reflecting minimal contribution of $\mathrm{Kv}$ channels in the total transmembrane current. On the contrary, during increased intravascular pressure, i.e. increased NSC current, model simulations predict varying degrees of the $\mathrm{Kv}$ channel opening, and an apparent difference in $\mathrm{V}_{\mathrm{m}}$ and $\mathrm{Ca}^{2+}$ dynamics among different groups. Simulations highlight the inhibitory role of $\mathrm{Kv}$ channel activation on the myogenic response of the cell. More specifically, more $\mathrm{Kv}$ density in CADASIL results in a greater negative feedback, which translates to more hyperpolarized $\mathrm{V}_{\mathrm{m}}$ compared to CTL, resulting in less activity of VDCC channels, lower $\left[\mathrm{Ca}^{2+}\right]_{\mathrm{i}}$ levels and subsequently less myogenic tone. Conversely, less negative feedback in SAH in response to lower number of active $\mathrm{Kv}$ channels results in more depolarized $\mathrm{V}_{\mathrm{m}}$, higher $\left[\mathrm{Ca}^{2+}\right]_{\mathrm{i}}$ levels, and more myogenic tone. Consistent with observation from control and diseased animal models, simulations predict a profound role of $\mathrm{Kv}$ channels in the regulations of the myogenic tone of arterioles, and consequently in the control of CBF. 
A

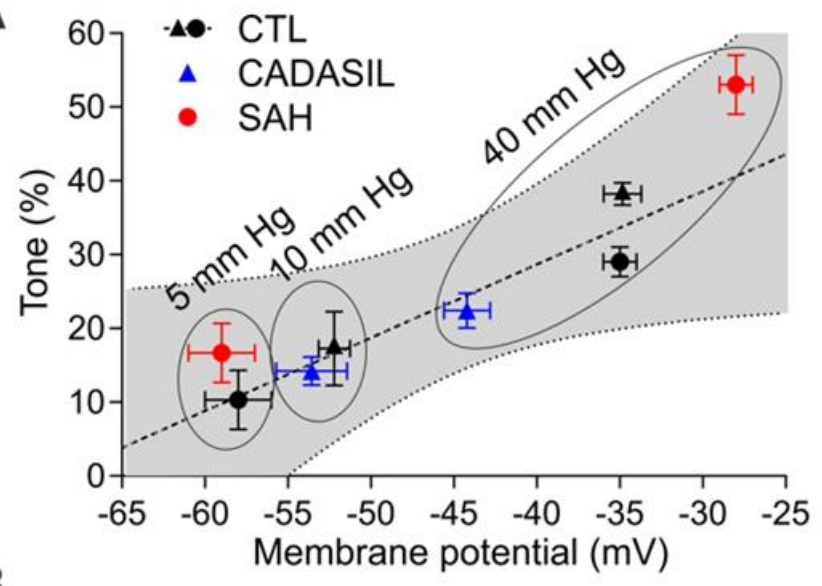

B

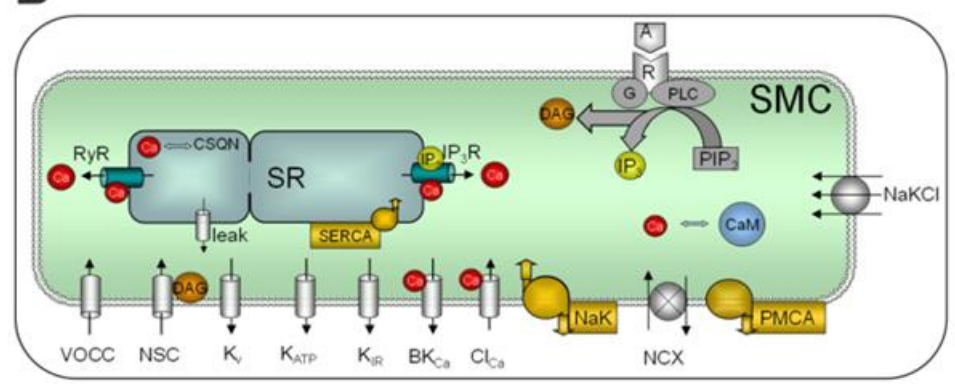

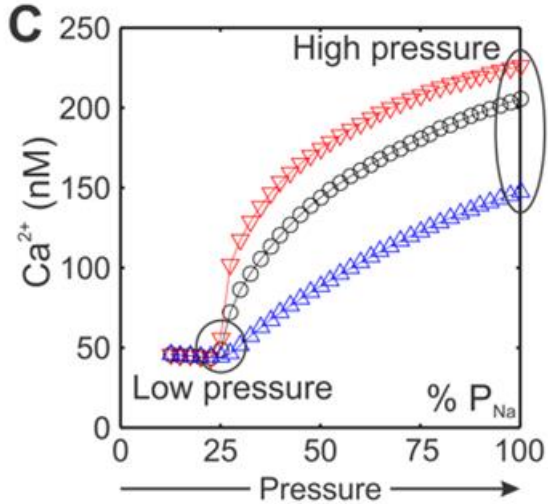

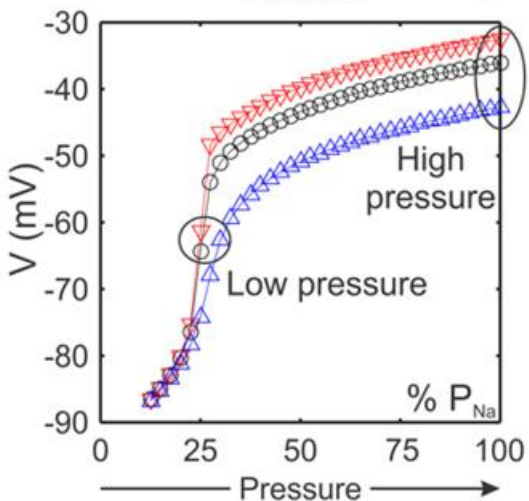

Figure 3-2 $\mathrm{V}_{\mathrm{m}}$ and myogenic tone relation in normal and diseased animal models. (A) Membrane potential and myogenic tone for CTL (black triangles: mice from [120] and black circles: rats from [144]), CADASIL, (blue triangles) and SAH (red circles) at different values of intraluminal pressure. (B) A detailed model of SMC electrophysiology, adapted from [145], was modified by incorporating the $K_{V}$ current of Figure 3-2, while other transmembrane currents were adjusted to produce resting $\mathrm{V}_{\mathrm{m}}$ and $\mathrm{Ca}^{2+}$ concentration in agreement with experimental data [120, 146].(C) Increased intravascular pressure was simulated by depolarizing SMC membrane through an elevated $\mathrm{Na}^{+}$permeability $\left(\mathrm{P}_{\mathrm{Na}}\right)$. Model simulations, in agreement with the corresponding experiments in (A), show differences between CADASIL, SAH, and CTL animals in $\mathrm{V}_{\mathrm{m}}$ (bottom panel) and $\mathrm{Ca}^{2+}$ (top panel) as pressure increases. [Parameters as in reference (102) except: $\mathrm{P}_{\mathrm{VDCC}}=6.3 \times 10^{-5} \mathrm{~cm} / \mathrm{s} ; \mathrm{P}_{\mathrm{NaNSC}}=1.23 \times 10^{-6} \mathrm{~cm} / \mathrm{s} ; \overline{\mathrm{I}}_{\mathrm{PMCA}}=8.58 \mathrm{pA} ; \overline{\mathrm{I}}_{\mathrm{NaK}}=7.76 \mathrm{pA} / \mathrm{pF} ; \mathrm{G}_{\mathrm{Kir}}=0.5 \mathrm{nS} /(\mathrm{mM})^{0.5}$; $\mathrm{G}_{\mathrm{Na}, \text { leak }}=0.12 \mathrm{nS} ; \mathrm{C}_{\mathrm{m}}=12.8 \mathrm{pF}$ ].

\subsection{IMPACT OF SMALL VESSEL PATHOLOGIES ON THE INTERPLAY}

\section{BETWEEN KV AND KIR CHANNELS DURING NVC}

As mentioned above, and also discussed in great depth in CHAPTER 2, Kir channels have a pivotal role in NVC. Here we use a representative PA SMC Kir current (obtained from fitting the experimental recording in [36]) to probe the interplay between the Kir and Kv channels during model increases in $\left[\mathrm{K}^{+}\right]_{0}$ associated with NVC. A rise in $\left[\mathrm{K}^{+}\right]$o causes a rightward and upward shift in the current-voltage relationship of Kir currents (Figure 3-3 
A, left inset), due to the increase in the channel conductance, as well as the shift in $\mathrm{E}_{\mathrm{K}}$ to more depolarized potentials [147]. In contrast, a modest increase in $\left[\mathrm{K}^{+}\right]_{\mathrm{o}}$ decreases the outward current of $\mathrm{Kv}$ channels owing to the reduced $\mathrm{K}^{+}$chemical gradient (Figure 3-3 A, right inset). Additionally, $\mathrm{V}_{\mathrm{m}}$ hyperpolarization in response to the activation of $\mathrm{Kir}$ channels in expected to further reduce Kv channel open probability (Figure 3-1). Thus, the opposing responses of these channels to model elevations of $\left[\mathrm{K}^{+}\right]_{\mathrm{o}}$ creates a "tug-of-war" dynamic that ultimately determines PA diameter during NVC. Under normal conditions, the influence of Kir currents to modest elevations of $\left[\mathrm{K}^{+}\right]_{\mathrm{o}}$ overcomes those of $\mathrm{Kv}$ currents, which leads to $\mathrm{SMC} \mathrm{V}_{\mathrm{m}}$ hyperpolarization and subsequent increase in the CBF.

Experimental evidence, however, indicates an impaired NVC in animal models with CADASIL and SAH $[118,122,148,149]$. To assess if the alteration in the Kv channel density in these pathologies is responsible for the observed impairment in NVC, we used the PA SMC model to predict the response of the system to increases in $\left[\mathrm{K}^{+}\right]_{\mathrm{o}}$ (from 3 to 8 $\mathrm{mM}$ ) for CTL and diseased models. The combined current-voltage curves of the representative Kir current and the three models of Kv currents in Figure 3-1, are shown for resting and elevated potassium concentrations (Figure 3-3 A) (solid lines are combined Kv and Kir currents at rest, and dashed lines are during the potassium stimulus). The relative magnitude of the aforementioned opposing influences at a given $\left[\mathrm{K}^{+}\right]_{\mathrm{o}}$ level, i.e. the dynamic "tug-of-war" between $\mathrm{Kv}$ and Kir currents, predicts a voltage window where the Kir influence outweighs that of $\mathrm{Kv}$ (the shaded regions in Figure 3-3 A), i.e., the "Kir window". Within this $\mathrm{V}_{\mathrm{m}}$ range, hyperpolarization can be achieved during potassium challenge; while, outside of the window, potassium stimulus would likely result in a 
depolarization of the cells since the influence of $\mathrm{Kv}$ is more pronounced than Kir. Figure 3-3 A illustrates that the increase in Kv channel density (from SAH to CADASIL) reduces the size of the Kir window and will shift the window to more hyperpolarized potentials; hence, reducing the ability of the cell to hyperpolarize during potassium stimulus. The corresponding response of the $\mathrm{V}_{\mathrm{m}}$ of PA SMC to potassium challenge is depicted in Figure 3-3 B. Consistent with predictions in Figure 3-3 A and Figure 3-2 A, CADASIL model shows a more hyperpolarized resting $\mathrm{V}_{\mathrm{m}}$ compared to CTL and $\mathrm{SAH}$ (since Kir window is shifted to the left). The level of hyperpolarization during the potassium challenge is increased from CADASIL to CTL, to SAH models (notice that the Kir window is more extended in SAH, compared to CTL, and CADASIL in Figure 3-3 A).

Model simulations for SAH, the red region in Figure 3-3 A, predicts an extended Kir window compared to CTL models owing to a decreased Kv density. Although this result is consistent with the observations that isolated PAs from SAH animals dilate in response to $\mathrm{K}^{+}$increase [149], evidence suggest an inversion of NVC in SAH models where vasoconstriction is observed, in both brain slices and in vivo, during neuronal activation [148-150]. This inversion, however, is postulated to be the results of an increase in basal $\left[\mathrm{K}^{+}\right]_{\mathrm{o}}$. Elevations in basal $\left[\mathrm{K}^{+}\right]_{\mathrm{o}}$ leads to a more depolarized SMC EK, due to the reduced $\mathrm{K}^{+}$chemical gradient, which most likely positions the $\mathrm{V}_{\mathrm{m}}$ of the SMC outside of the Kir window. Thus, under these conditions the cell is expected to depolarize, which consequently results in enhanced Ca2+ entry through VDCCs and vasodilation [148-150]. 

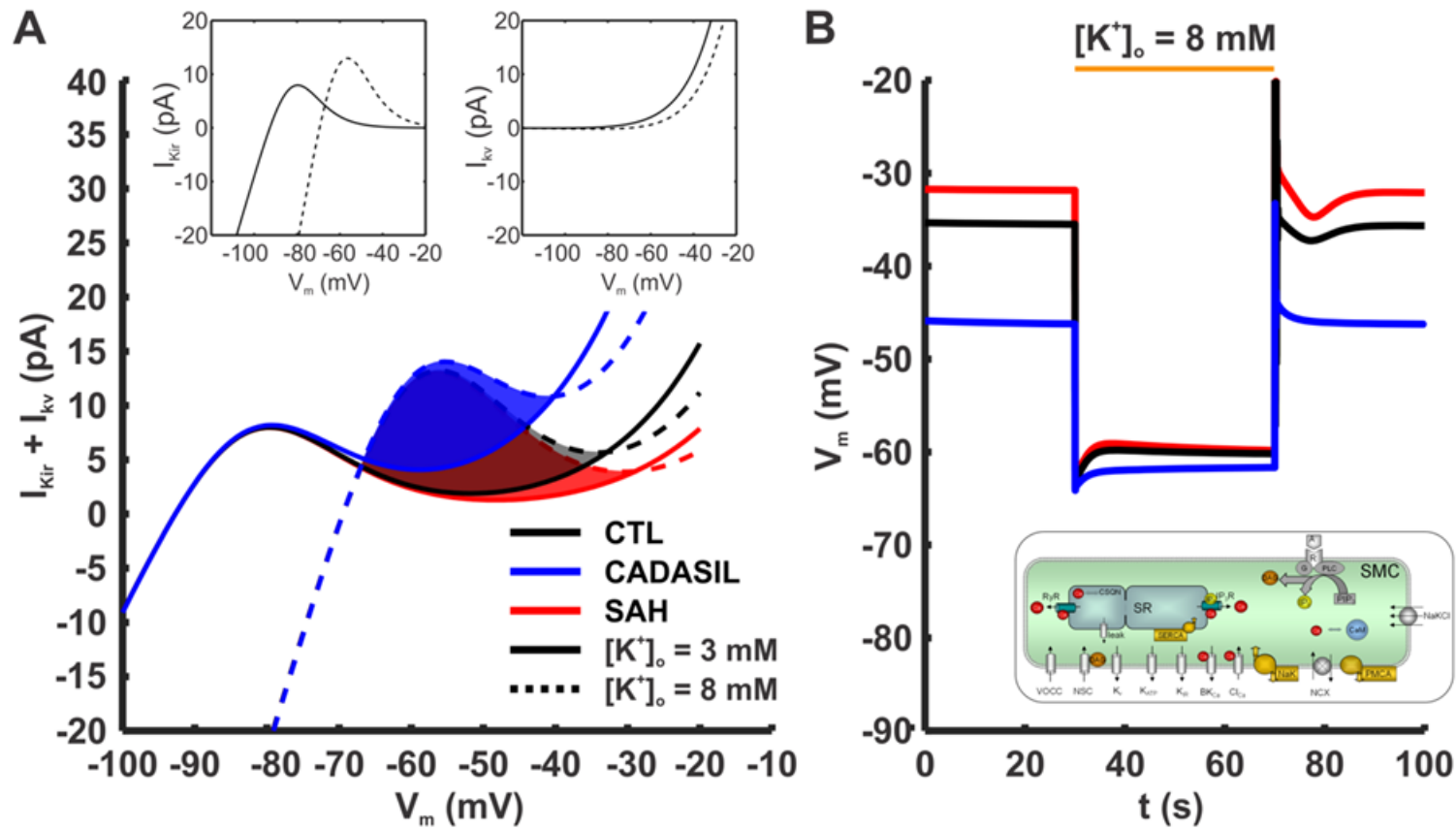

Figure 3-3 Interplay between $\mathrm{Kv}$ with Kir currents in determining $\mathrm{V}_{\mathrm{m}}$ dynamics at rest and during a potassium challenge. (A) Combined contribution of Kir and $\mathrm{K}_{\mathrm{v}}$ currents in healthy and diseased models during rest and $\left[\mathrm{K}^{+}\right]_{\mathrm{o}}$ stimulus. Solid lines: the sum of the two currents at rest, and dashed lines during elevation of $\left[\mathrm{K}^{+}\right]_{\mathrm{o}}$ from 3 to $8 \mathrm{mM}$. The shaded regions show the range of voltages where Kir influence dominates the $\mathrm{Kv}$ current, i.e. the $\mathrm{V}_{\mathrm{m}}$ window where the $\mathrm{K}^{+}$challenge will result in hyperpolarization. As $\mathrm{K}_{\mathrm{V}}$ current density increases (from SAH, red lines; to CTL black lines; to CADASIL, blue lines) the window shrinks in size and shifts to more hyperpolarized potentials. (B) Representative simulations using the model of PA SMCs from Figure 3-3 to increases in $\left[\mathrm{K}^{+}\right]_{\mathrm{o}}$. Compared to SAH and CTL models, the change in membrane potential is less for CADASIL as a result of the more hyperpolarized resting $\mathrm{V}_{\mathrm{m}}$ prior to the $\mathrm{K}^{+}$challenge. $\left[\mathrm{G}_{\mathrm{Kir}}=0.76\right.$ $\left[\mathrm{nS} /(\mathrm{mM})^{0.5}\right] ; \mathrm{k}_{\mathrm{Kir}}=7[\mathrm{mV}] ; \mathrm{V}_{\mathrm{Kir}, 0.5}=\mathrm{E}_{\mathrm{K}}+12[\mathrm{mV}]$.

\subsection{CONCLUSION}

Consistent with experimental data, model simulations presented here corroborated the importance of $\mathrm{Kv}$ channels in regulating the resting $\mathrm{V}_{\mathrm{m}}$ and myogenic tone of PAs. Model results successfully predicted how the changes in the density of $\mathrm{Kv}$ channels can profoundly impact the dynamics of $\mathrm{V}_{\mathrm{m}}$ and intracellular calcium. With the help of model simulations, we identified a potential "tug-of-war" dynamic between the activity of Kir and $\mathrm{Kv}$ channels, which can determine conditions where $\mathrm{K}^{+}$stimulation would result in either hyperpolarization or depolarization of PA SMCs. Model simulations suggest that $\mathrm{Kv}$ upregulation in CADASIL narrows the Vm window where Kir can overcome the 
counteracting effect of $\mathrm{Kv}$ channels, which consistent with experimental data, results in more hyperpolarized $\mathrm{V}_{\mathrm{m}}$ and reduced level of hyperpolarization. In $\mathrm{SAH}$, a more extended Kir window was predicted, which led to more depolarized resting $V_{m}$ in increased hyperpolarization level during modest elevation in $\left[\mathrm{K}^{+}\right]_{\mathrm{o}}$.

In CADASIL, the decreased myogenic tone and more hyperpolarized resting membrane potentials compared to control animal models resulted in the attenuation of vasoconstriction. On the contrary, due to the elevated myogenic tone and more depolarized membrane potentials, animal models in SAH showed increased vasoconstriction and decreased CBF compared to control. Simulations performed in this chapter provided a plausible mechanistic understanding of these experimentally observed alterations in the myogenic tone and resting membrane potential of PA SMCs during small vessel pathologies. Simulation results indicated that the interplay between the Kir and $\mathrm{Kv}$ channels is critical in the myogenic tone and the response of the cell to modest elevations in $\left[\mathrm{K}^{+}\right]_{\mathrm{o}}$. Simulations predict that restoring Kir to $\mathrm{Kv}$ current balance can be a prospective therapeutic strategy in the treatment/prevention of small vessel disease, particularly in the case of CADASIL and SAH. 


\section{CHAPTER 4 A NEW CURRENT SOURCE DENSITY ANALYSIS METHOD FOR PROPAGATING SOURCES IN THE BRAIN: APPLICATION TO CORTICAL SPREADING DEPRESSION}

The content of this chapter is published in Journal of Neuroscience Methods as Moshkforoush A, Valdes-Hernandez PA, Rivera-Espada DE, Mori Y, Riera J. "waveCSD: A method for estimating transmembrane currents originated from propagating neuronal activity in the neocortex: Application to study cortical spreading depression" [151].

\subsection{INTRODUCTION}

Development of high-resolution, multi-contact microelectrode arrays (MEA), have revolutionized methods for estimation of transmembrane currents from their extracellular local field potential (LFP) reflections. This estimation procedure is known as current source density (CSD) analysis. CSD analysis methods have recently been expanded to multidimensional domains (2D - $[152,153], 3 \mathrm{D}$ - [153-156]); however, the most widely used application is to determine CSDs associated with neocortical columns using linear arrays [153, 157-161]. For a detailed review of CSD analysis methods and their applications, refer to [162].

Methods developed for neocortical CSD analysis using linear arrays assume that CSDs vary only as a function of cortical depth ( $z$-axis) and that their tangential $(x-y)$ extension can be either infinite [159] or confined to a circular disk of a predefined radius around the 
electrodes-the inverse CSD method (iCSD [161]) and the kernel CSD (kCSD) method [153]. The layer-based and columnar organization of the mammalian neocortex is the physiological foundation for the tangential (laminar) symmetry in the aforementioned methods for CSD analysis. This assumption is suitable for most experimental paradigms where neuronal activations are expected to localize in specific functional structures of neocortex, and CSD analysis results have significantly aided in the determination of neuronal circuit organization in sensorial $[155,163]$, motor $[164,165]$ and cognitive $[166]$ cortical regions.

However, in several experimental conditions, neuronal activity propagates continuously along large areas of the neocortical sheet. Alpha-rhythm waves are reported to originate in the occipital regions and propagate progressively to frontal regions at a velocity of $\sim 30$ cm/s [167-169]. Global traveling waves are also reported in studies using scalp EEG [170173], as well as in studies with direction measurements using optical imaging techniques [174]. Cortical spreading depression, a well-studied phenomenon associated with migraine aura, is another example where an electro-diffusional wave propagates along the cortical mantle at a reported velocity of $3-5 \mathrm{~mm} / \mathrm{min}[175,176]$. These examples along with recent discoveries of traveling waves in visual perception [177] and sensorimotor integration [178], call for the development of novel CSD analysis methods accounting for the propagating nature of neuronal activity. For more information about cortical travelling waves refer to [179].

In this study, we used two mathematical constructs to develop a new method (waveCSD) for CSD analysis of experimental applications where a planar CSD wave is propagating 
along the neocortex with a constant velocity. Similar to the iCSD method, our waveCSD method works based on the inversion of the electrostatic forward model of LFP generation. First, we expand the planar wave in cubic spline bases and assume compact support in the direction of propagation. Second, to account for the ill-conditioning of the forward problem discrete kernel, we use Tikhonov regularization scheme. The use of regularization techniques has been shown to significantly improve the reconstruction accuracy, especially in the presence of noise in the voltage data [153]. Using simulations, we evaluate the sensitivity of waveCSD to the observation noise, the electrode spatial resolution, and the uncertainties in the knowledge of the planar wave velocity. We also compare the reconstruction accuracy of waveCSD with spline-iCSD [161], and kCSD [153] methods adapted to the reconstruction of CSDs emerging from the propagation of neuronal activity. Finally, we apply the waveCSD method to determine neocortical transmembrane ionic currents associated with cortical spreading depression in rats.

\subsection{MATERIALS AND METHODS}

\subsubsection{CSD analysis of a planar wave of neocortical neuronal activity (waveCSD method)}

Assuming constant and isotropic tissue conductivity, the relationship between the current source density in the brain, denoted by $C$, and the generated LFPs, denoted by $V$, is determined by the Poisson equation $[159,180]$ :

$\sigma \Delta V=-C$

Equation 4-1

Assuming an infinite medium, the solution to Equation 4-1 at an electrode position $\overrightarrow{r_{e}}=$ $\left[\begin{array}{lll}x_{e} & y_{e} & z_{e}\end{array}\right]$ is provided by: 


$$
V_{e}(t)=\frac{1}{4 \pi \sigma} \iiint \frac{C(x, y, z, t)}{\sqrt{\left(x-x_{e}\right)^{2}+\left(y-y_{e}\right)^{2}+\left(z-z_{e}\right)^{2}}} d x d y d z
$$

Here, we propose a method for simulating an experimental setup in which a wave of neuronal activity, originated in a location $\left(\overrightarrow{r_{0}}\right)$, is moving tangentially along the cortex with a constant velocity $v$, and a linear probe is perpendicularly inserted into the neocortex along the $z$ direction to record the resultant LFP. We assume that $\overrightarrow{r_{0}}$ is far enough from the electrode array to render the propagating wave planar. In this model, the propagation direction is along the $x$-axis, thereby allowing us to drop the $y$-dependency of $C$ in Equation 4-2:

$$
\begin{aligned}
& V_{e}(t)=\frac{1}{4 \pi \sigma} \iint K^{e}(x, z) C(x, z, t) d x d z \\
& K^{e}(x, z)=\int_{y_{s}}^{y_{f}} \frac{d y}{\sqrt{\left(x-x_{e}\right)^{2}+\left(y-y_{e}\right)^{2}+\left(z-z_{e}\right)^{2}}} \\
& \quad=\left.\log \left[\frac{1}{\left(y_{e}-y\right)+\sqrt{\left(x-x_{e}\right)^{2}+\left(y-y_{e}\right)^{2}+\left(z-z_{e}\right)^{2}}}\right]\right|_{y_{s}} ^{y_{f}}
\end{aligned}
$$

where $y_{f}=-y_{s}=\alpha$ are the limits of the cortex in the $y$ direction assuming $y_{0}=0$. Since the propagation velocity is assumed constant, the CSD profile at any $x$ location can be written using the d'Alembert's formula:

$$
C(x, z, t)=C(x-v t, z)
$$

Note that we only study propagation of the wave towards the electrodes. Assuming that the CSD has a spatial compact support in the $x$ direction at any given time instance $t$, the $x$ integration in Equation 4-3 can be simplified to: 


$$
V_{e}(t)=\frac{1}{4 \pi \sigma} \iint_{x_{S}(t)}^{x_{f}(t)} K^{e}(x, z) C(x-v t, t) d x d z
$$

where

$$
\begin{aligned}
& x_{s}(t)=x_{0}+v t \\
& x_{f}(t)=x_{s}(t)+L
\end{aligned}
$$

$x_{0}$ is the $x$ location of the wave at time $t_{0}=0$ (here we set $x_{0}=0$ ), and $L$ is the assumed length of the wave in the $x$ direction, whose shape will be defined using cubic splines. Using a change of variables (i.e. $\tau=x-v t$ ), Equation 4-6 can be reorganized into:

$$
V_{e}(t)=\frac{1}{4 \pi \sigma} \iint_{0}^{L} K^{e}(v t+\tau, z) C(\tau, z) d \tau d z
$$

Upon discretizing $C(\tau, z)$ in a two-dimensional rectangular grid for $\tau$ and $z$ via bicubic splines, the linearized version of Equation 4-8, for each time instance $t$, is written as follows:

$$
\mathbf{v}=\mathbf{Q} \mathbf{c}+\xi
$$

where $\mathbf{v}=\left[\begin{array}{llll}\boldsymbol{\varphi}^{T}\left(\mathrm{t}_{0}\right) & \ldots & \boldsymbol{\varphi}^{T}\left(\mathrm{t}_{n}\right)\end{array}\right]^{T}, \quad \boldsymbol{\varphi}\left(\mathrm{t}_{i}\right)=\left[\begin{array}{llll}V_{1}\left(\mathrm{t}_{i}\right) & \ldots & V_{n_{e}}\left(\mathrm{t}_{i}\right)\end{array}\right]^{T}$ and $\mathbf{c}$ is a column vector of the CSD values to be determined at the grid points (here we assume $n_{\tau}$ and $n_{z}$ points in the $\tau$ and $z$ directions, respectively). The derivation of Equation 4-8 and elements of $\mathbf{Q}$ is outlined in Appendices A4 and B4. We have added Gaussian noise $\xi \sim N\left(\mathbf{0}_{n_{e} \times 1}, \sigma_{n}^{2} \mathbf{I}_{n_{e}}\right)$, where $n_{e}$ is the number of electrodes, to Equation 4-9 to account for uncorrelated instrumental noise in the recordings with standard deviation $\sigma_{n}$. In the simulations of this chapter, we quantify $\sigma_{n}$ as percentages of the maximum absolute value of the LFP. 
The inverse problem associated with Equation 4-9, i.e. estimating $\mathbf{c}$ given $\mathbf{v}$, is both illposed and ill-conditioned. Thus, we use the Tikhonov regularization scheme [181] for estimation of current sources $\hat{\mathbf{c}}$ :

$$
\hat{\mathbf{c}}=\left(\mathbf{Q}^{\mathrm{T}} \mathbf{Q}+\lambda^{2} \boldsymbol{\Gamma}^{\mathrm{T}} \boldsymbol{\Gamma}\right)^{-1} \mathbf{Q}^{\mathrm{T}} \mathbf{v}
$$

where $\boldsymbol{\Gamma}=\mathbf{I}_{n_{e}}$ is the Tikhonov matrix and $\lambda$ is the optimal regularization parameter, determined via generalized cross-validation (GCV) method [182] through minimization of the following evaluation function $(E(\lambda))$ :

$$
\mathrm{E}(\lambda)=\frac{\|\boldsymbol{P}(\lambda) \mathbf{v}\|^{2}}{\operatorname{tr}(\boldsymbol{P}(\lambda))^{2}}
$$

where $\boldsymbol{P}(\lambda)$ is the projection matrix defined as:

$$
\boldsymbol{P}(\lambda)=\mathbf{I}-\mathbf{Q}\left(\mathbf{Q}^{\mathrm{T}} \mathbf{Q}+\lambda^{2} \mathbf{I}\right)^{-1} \mathbf{Q}^{\mathrm{T}}
$$

Equation 4-12

The estimation of other model parameters, i.e. $v$ and $\alpha$, from the LFP data is also done using Equation 4-12. More specifically, a wide range of $v$ and $\alpha$ values will be chosen for a given LFP recording. For any pair of $v$ and $\alpha$ values, i.e., $\left(v_{i}, \alpha_{j}\right)$, the corresponding $\mathbf{Q}$ matrix will be calculated and the regularization parameter $\lambda_{i j}$ will be estimated. The pair which has the lowest GCV score, i.e., $E_{i j}\left(\lambda_{i j}\right)$, will be chosen as the optimal model parameters. In this chapter, we denote the CSD profile reconstructed using the waveCSD method as $\mathbf{c}_{\text {waveCSD }}$.

\subsubsection{CSD analysis of a propagating wave using iCSD and kCSD methods}

iCSD [161] is the pioneering method which utilized the inverse problem of Equation 4-2 for the CSD analysis of LFP recordings using multielectrode arrays with various 
configurations. In the original article by Pettersen et al. [161], three model-based iCSD methods (i.e., step-, delta-, and spline-iCSD) were proposed and applied to the LFP data from linear (laminar) probes. The authors also developed iCSD methods for off-center electrode placements. In this study, all comparisons will be made with the spline-iCSD method centered at the electrodes. The assumption in this method is that the CSD is constant in the tangential direction and is confined to a cylinder of a predefined diameterusually the size of a cortical column — around the electrodes. Potworowski and coworkers [153] further generalized the iCSD method to kCSD method, a non-parametric CSD analysis methodology which utilizes reproducing kernel Hilbert spaces for the reconstruction of current source densities which can be applied to various configuration of recording electrodes. Major improvements in the kCSD method compared to iCSD are: I) incorporation of regularization algorithms to improve the reconstruction accuracy in the presence of observation noise in voltage data, and II) estimation of model parameters using k-fold cross-validation algorithm from the LFP recordings. The kCSD method also assumes that current sources are confined in a cylinder, and that the tangential extension of the sources are either defined using a step function, or a Gaussian profile. Here, we use the Gaussian profile of current sources in the 1D kCSD method for all comparative simulations.

Throughout this chapter, we refer to spline-iCSD and 1D kCSD as iCSD and kCSD, respectively. Application of either $\mathrm{iCSD}$ or $\mathrm{kCSD}$ method to LFP gives a temporal distribution of the variation of CSDs along the $z$ direction, here denoted as $\mathbf{c}_{i C S D}^{t}(t, z)$ and $\mathbf{c}_{k C S D}^{t}(t, z)$, respectively. Neither method is intended to account for propagating CSD 
waves originating far from the electrodes and covering large-scale neocortical distances. Hence, to be able to interpret the solution of $\mathrm{iCSD}$ and KCSD methods as a wave of length $L$ propagating towards the electrode with a constant velocity $v$, these temporal maps should be converted to spatial propagating profiles, denoted as $\mathbf{c}_{i C S D}^{S}(x, z)$ and $\mathbf{c}_{k C S D}^{S}(x, z)$, respectively. This conversion, which allows for the comparison of accuracy of reconstruction of $\mathrm{iCSD}$ and $\mathrm{kCSD}$ with wave $\mathrm{CSD}$, is done using a linear transformation of the $\mathbf{c}_{i C S D / k C S D}^{t}$ profile as follows:

$$
\begin{gathered}
\mathbf{c}_{i C S D / k C S D}^{S}(x, z)=\mathbf{c}_{i C S D / k C S D}^{t}\left(x_{e}-v t, z\right) \\
t \in\left[\left(x_{e}-L\right) / v, x_{e} / v\right]
\end{gathered}
$$

Equation 4-13

The up and running version of the iCSD method (CSDPlotter) can be downloaded from (http://web.eng.fiu.edu/jrieradi/CSDPlotter/).

\subsubsection{Reconstruction accuracy measures}

Using simulations, we compare the reconstruction accuracy between waveCSD, iCSD and kCSD methods. A known CSD profile ( $\mathbf{c}_{\text {ground-truth }}$ ) of length $L$, propagating towards the electrodes at a constant velocity $v$, was used to generate simulated LFP data using the forward model of Equation 4-8. At any desired Gaussian noise level, 50 distributions of the noisy simulated LFP were constructed (random number generator seeding was shuffled to assure different noise distributions in each trial), and source reconstruction was performed using all three methods for all trials. It should be noted that noise filtering in the iCSD method is achieved by applying a Gaussian spatial filter on the estimated CSD from unfiltered LFP recordings [161]. The model parameters for iCSD (the diameter of the cylinder $(d)$ ), and $\operatorname{kCSD}$ (the cylinder radius $(R)$ and thickness $(h)$ of the Gaussians) 
methods were selected using the following approach: Firstly, k-fold cross-validation algorithm implemented in the kCSD method is applied to the LFP data to choose optimal $R$ and $h$. Secondly, since both iCSD and kCSD methods work under the same assumption that current sources are confined in a cylinder, and that parameter estimation is not included in the original iCSD method, $d$ in the iCSD method was chosen same as that of $\mathrm{kCSD}$ method, i.e., $d=2 R$. The effect of changing $d$ in the reconstruction using iCSD method was also separately studied. We calculate the accuracy of a given method by comparing its reconstructed profile, $\mathbf{c}_{\text {reconstructed }}=\left\{\mathbf{c}_{\text {waveCSD }}, \mathbf{c}_{i C S D}^{S}, \mathbf{c}_{k C S D}^{S}\right\}$, with $\boldsymbol{c}_{\text {ground-truth }}$, using the Relative Difference Measure (RDM) and the Magnitude (MAG) ratio. The RDM primarily quantifies differences in shape between the profiles, while MAG quantifies their relative magnitude [183].

$$
\begin{array}{ll}
\boldsymbol{R D M}\left(\mathbf{c}_{\text {reconstructed }}, \mathbf{c}_{\text {ground-truth }}\right)=\left\|\frac{\mathbf{c}_{\text {reconstructed }}}{\left\|\mathbf{c}_{\text {reconstructed }}\right\|}-\frac{\mathbf{c}_{\text {ground-truth }}}{\left\|\mathbf{c}_{\text {ground-truth }}\right\|}\right\| \in[0,2] & \text { Equation 4-14 } \\
\boldsymbol{M A G}\left(\mathbf{c}_{\text {reconstructed }}, \mathbf{c}_{\text {ground-truth }}\right)=\| \frac{\mathbf{c}_{\text {reconstructed }}}{\mathbf{c}_{\text {ground-truth }} \|} & \text { Equation 4-15 }
\end{array}
$$

The condition where $\mathrm{RDM}=0$ and $\mathrm{MAG}=1$ indicates identical profiles and optimal accuracy.

\subsubsection{Recording LFP data during cortical spreading depression}

A total of five rats were used in this study to record LFPs using an A1x32 linear probe during cortical spreading depression. All procedures were conducted in agreement with the Institutional Animal Care and Use Committee at Florida International University (IACUC 15-014). 


\subsubsection{Animal preparation and cortical spreading depression induction}

A mixture of 5\% isoflurane and pure oxygen was supplied to a hermetically sealed box (1 $\mathrm{L} / \mathrm{min}, 14.7 \mathrm{psi}$ ) to induce anesthesia in rats. For each rat, the scalp was shaved, and the rat was fixed to a stereotaxy (Narishige, Japan). The isoflurane level was reduced to 1.75$2.50 \%$ to stabilize the breathing rate to $50-55$ breaths per minute. The scalp and the underlying connective tissue were removed to set the positions for the burr holes (for reference and ground electrodes), and the craniotomies (for cortical spreading depression induction and probe insertion). Burr holes were made approximately $1 \mathrm{~mm}$ posterior to lambda and the reference and ground electrodes were placed in direct contact with the intracranial spinal fluid. The two craniotomies, one $2 \mathrm{~mm}$ anterior and the other $4 \mathrm{~mm}$ posterior to Bregma, were positioned on the right hemisphere approximately $2 \mathrm{~mm}$ lateral from the midline. The posterior craniotomy was used for induction of cortical spreading depression, and the anterior craniotomy was used for LFP recording using the linear (A1x32) probe. The probe was perpendicularly inserted approximately $1.7 \mathrm{~mm}$ into the neocortex using a micromanipulator control system (MPC-200/ROE 200/MPC-385/MPC325 Sutter Instruments). The accurate positioning and depth of probe penetration was histologically verified postmortem. The cortical spreading depression events were induced via topical application of $\sim 20-40 \mu \mathrm{l}$ of $1 \mathrm{M}$ solution of potassium acetate $(\mathrm{CH} 3 \mathrm{CO} 2 \mathrm{~K})$ in distilled water (Milli-Q, Millipore Corp.). The leakage of $\mathrm{CH} 3 \mathrm{CO} 2 \mathrm{~K}$ to the recording craniotomy was prevented using a dental cement barrier between the two craniotomies. The concentration of isoflurane anesthesia was reduced $(0.25-0.5 \%, 1 \mathrm{~L} / \mathrm{min}$ pure $\mathrm{O} 2,14.7$ psi), and was supplemented with intraperitoneally-administered Dexmedetomidine hydrochloride (Dexdomitor, $0.25 \mathrm{mg} / \mathrm{kg}$ ), to ensure that the spontaneous cortical brain 
activity was minimally compromised The efficacy of this approach was previously tested and verified by our group [184]. Throughout the recording, rat physiology (i.e. body temperature $\left(\sim 37{ }^{\circ} \mathrm{C}\right)$ and respiration rate ( 28 breaths per minute) was constantly monitored using the PowerLab 8/35 data acquisition device and the LabChart software (ADInstruments). The rat's body temperature was kept at the desired range using a heating pad (TPZ-0510EA, Texas Scientific Instruments, LLC) with a water-circulating pump (TP700, Texas Scientific Instruments, LLC).

\subsubsection{Intracranial recordings}

Two recordings, 30 minutes each, were performed for each rat. At the end of the first recording, the posterior craniotomy was washed with saline and dried before the start of the second recording. The spontaneous brain activity was recorded for 5 minutes before the $\mathrm{CH} 3 \mathrm{CO} 2 \mathrm{~K}$ solution was administered to the posterior craniotomy. The LFPs were recorded with a PZ5 AC amplifier at $25 \mathrm{kHz}$ (Tucker \& Davis Technologies, TDT). The amplifier was connected to a signal processing unit (RZ2, TDT) by an optical fiber, and to a preamplifier by a coaxial cable. The OpenEx software (TDT) was used for recordings. The raw data was notch-filtered in MATLAB to remove the $60 \mathrm{~Hz}$ artifacts, and a lowfrequency $(1-170 \mathrm{~Hz})$ Butterworth IIR bandpass filter was used to extract the LFPs from the raw data.

\subsection{5 $\quad c_{\text {waveCSD }}$ associated with cortical spreading depression}

CSD analysis was performed on LFPs obtained from rats during cortical spreading depression using the waveCSD method. In order to check if individual CSD reconstructions exhibit a consistent and reproducible pattern across rats, the grand average CSD profile 
was obtained after nonlinear coregistration of the reconstructed CSDs. This was done through warping the reconstruction using a landmark-based nonlinear registration method based on the Approximate Thin Plate Splines (TPS) transformation outlined in [185]. For each individual, each landmark was chosen to represent a particular feature of visible contrast of the CSD reconstruction common to all individuals. Besides, their topological order was preserved across individuals to avoid folding of the transformations, i.e. negative values of their Jacobian. Since the TPS transformations are infinitely differentiable and no folding occurs, we ensure they capture the inter-individual diffeomorphic variability of rat neocortex expected in a group of normal rats.

\subsubsection{Statistical analysis}

Results are shown as mean \pm std, unless stated otherwise. The normality of the distribution of the accuracy measures (RDM and MAG) across methods (i.e. wave CSD, iCSD, and $\mathrm{kCSD})$ at different noise levels is tested using the Kolmogorov-Smirnov (KS) test. Since all distributions failed the KS test (data not shown here), a BoxCox transformation [186] is applied to the difference of the RDM and MAG measures across methods and a paired t-test is used to analyze if the difference is significant.

\subsection{RESULTS}

\subsubsection{Application of wave CSD, iCSD, and kCSD emthods to neocortical planar waves of neuronal activity}

To study the accuracy of waveCSD method in the reconstruction of propagating current sources compared to iCSD and kCSD methods, an arbitrary ground-truth profile waveform of CSDs (Figure 4-1, top left panel) was assumed to propagate towards a 16-electrode 
laminar probe, positioned $6 \mathrm{~mm}$ away, with a constant velocity of $4 \mathrm{~mm} / \mathrm{min}$. This waveform, containing non-compensated, oblique sink and source current densities, was used in all simulations performed in this chapter, unless stated otherwise. Comparative simulations for two other ground-truth profile examples are also provided in Appendix C.

4.
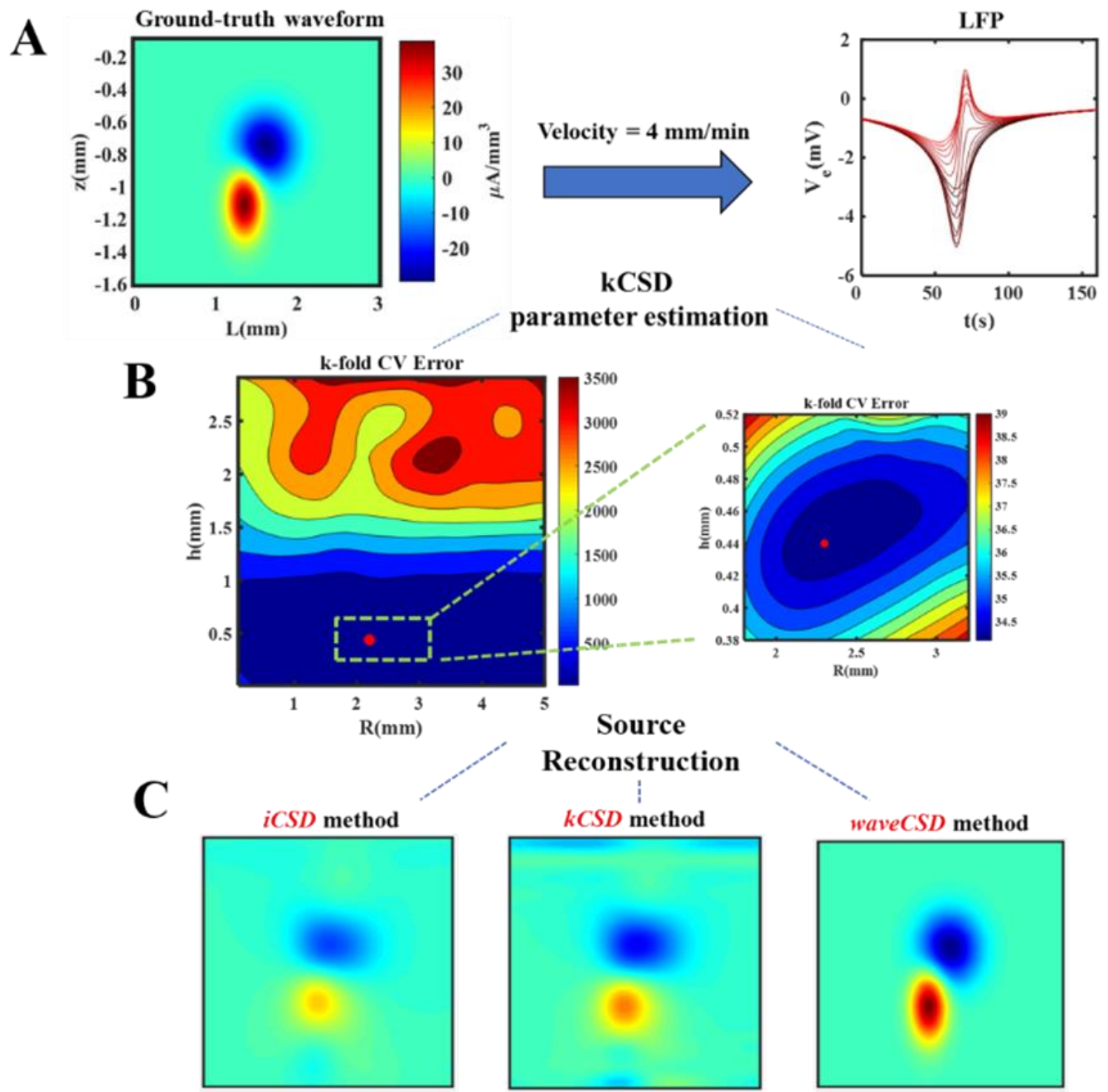

parameter estimation
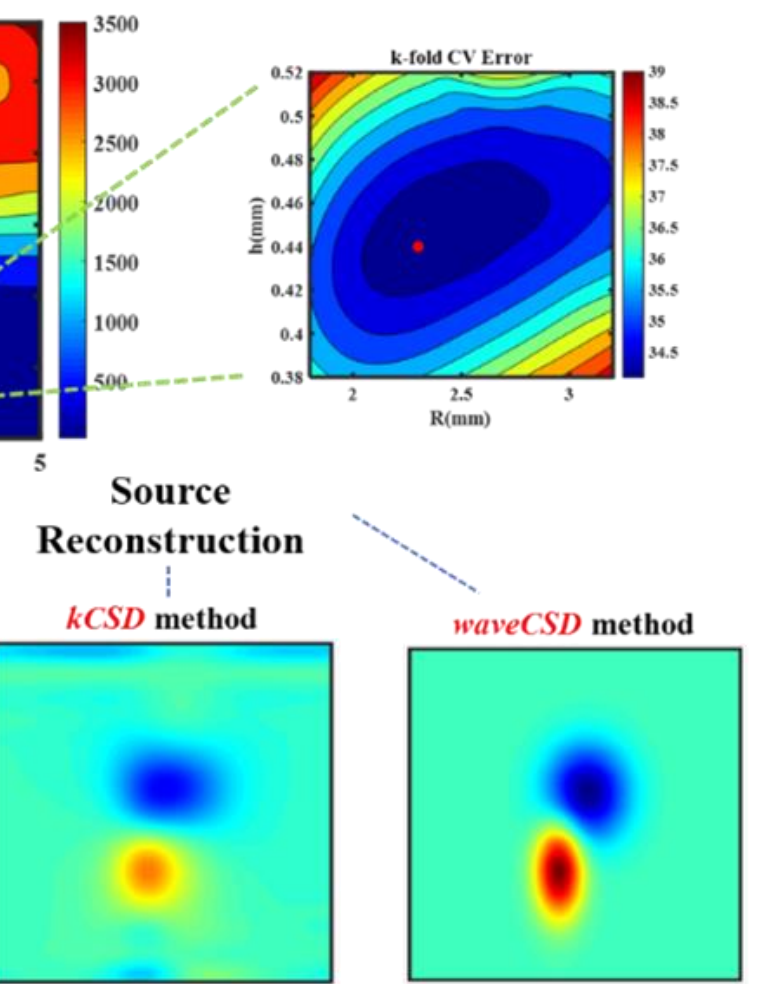

Figure 4-1 Comparison of the accuracy of waveCSD, iCSD, and kCSD methods in the reconstruction of LFPs generated from a ground-truth waveform. A) A ground-truth waveform (the CSD profile, top left) is assumed to be generated $6 \mathrm{~mm}$ away from an A1x16 probe, inserted perpendicularly in the cortex with an interelectrode spacing of $100 \mu \mathrm{m}$. The first electrode is assumed to be $100 \mu \mathrm{m}$ deep in the brain tissue. Here, the 
waveform propagates towards the electrodes at a constant velocity of $4 \mathrm{~mm} / \mathrm{min}$. The resultant LFPs are shown in the top right panel (black $\rightarrow$ most superficial electrode, and red $\rightarrow$ deepest electrode in the probe). B) Model parameter estimation for kCSD method using k-fold cross-validation technique as described in [153]. The left panel shows cross-validation error for a wide range of $\mathrm{R}$ and $\mathrm{h}$ values, and the inset confirms that the selected parameters (the red dot) coincide with the global minimum of cross-validation error values. The estimated diameter (2R) for the kCSD method is used for source reconstruction in the iCSD method shown in panel C. C) Source reconstruction using iCSD, kCSD and waveCSD methods without the presence of noise in the LFP data. (iCSD- RDM: 0.47, MAG: 0.67; kCSD- 0.47, MAG: 0.88, waveCSD- RDM: 0.01, MAG: 0.99). Both RDM and MAG measures confirm that the waveCSD method provides more accurate CSD reconstruction among all methods (i.e. RDM closer to zero and MAG closer to 1 in the waveCSD method). For comparison purposes, colorbar ranges and labels are the same as in 1 A left panel. Parameters: waveCSD method- The temporal resolution of the $\mathbf{Q}$ matrix is $1[\mathrm{~s}] . \mathrm{n}_{\tau}=40 ; \mathrm{n}_{\mathrm{z}}=30 ; \mathrm{L}=3[\mathrm{~mm}] ; \sigma=0.3$ $[\mathrm{S} / \mathrm{mm}] ; \alpha=3.5[\mathrm{~mm}]$. kCSD method- $\mathrm{R}=2.2[\mathrm{~mm}] ; \mathrm{h}=0.44[\mathrm{~mm}]$; number of sources $(\mathrm{nSrc})=320 . \mathrm{iCSD}$ method- $\mathrm{d}=4.4[\mathrm{~mm}]$.

The resultant forward solution to Equation 4-6 (i.e. the LFP distributions in the electrodes) is depicted in Figure 4-1 A top right panel. As described in Section 4.2.3 and illustrated in Figure 4-2 B, optimal model parameters of the kCSD method were estimated from the LFP data via k-fold cross-validation technique and are used for the source reconstruction using both kCSD and iCSD models. All methods were utilized to reconstruct the waveform from the LFP data without the presence of noise (Figure 4-1 C). The RDM and MAG measures indicate that waveCSD was more accurate than $\mathrm{iCSD}$, and $\mathrm{kCSD}$. Additionally, results indicate that while RDM measure of both $\mathrm{kCSD}$ and $\mathrm{iCSD}$ were identical for the given waveform (RDM - 0.47), kCSD performed better in terms of MAG measure in comparison with iCSD (iCSD- MAG: 0.67; kCSD - MAG: 0.88).

Figure 4-2 shows that waveCSD outperforms iCSD and $\mathrm{kCSD}$ for a wide range of observation noise in the LFP data (Figure 4-2). As illustrated in Figure 4-2 A, although increasing for all methods, the RDM measure is lower for wave CSD than for $\mathrm{ACSD}$ and $\mathrm{kCSD}$. We tested if this difference was statistically significant. Since the normality of the distribution of the RDM measure across trials failed the Kolmogorov-Smirnov (KS) test, we applied the BoxCox transformation to the difference between the RDM values of the 
wave CSD method with $\mathrm{iCSD}$ and $\mathrm{kCSD}$ methods. A paired t-test rejected the null hypothesis of zero difference for all noise levels with $\mathrm{p} \approx 0$. With this negligible $\mathrm{p}$-value we decided there was no need for any further statistical analysis in the following simulations. On the other hand, Figure 4-2 B depicts the MAG measures for waveCSD decreases with increasing noise levels, while it increases for both iCSD and kCSD. For the given CSD example waveform at levels of noise below 10\%, waveCSD is more accurate (i.e., MAG 1) than iCSD and kCSD. In the interval $~ 10-20 \%$, MAG values intersect, being close to 1 for all methods. In this interval, the MAG values for iCSD and kCSD are closer to 1 around the $20 \%$ noise level, compared to waveCSD. At higher noise levels, all methods diverge from the optimal MAG value. Two examples of source reconstruction using all three methods are provided in Figure 4-2 C and Figure 4-2 D for 10\% and 30\% noise levels, respectively. These examples further confirm that the inclusion of regularization techniques in both $\mathrm{kCSD}$ and waveCSD methods improves the quality of source reconstruction compared to iCSD method, particularly at higher percentages of noise in the data. For all the simulations in Figure 4-2, the wave velocity was fixed to $4 \mathrm{~mm} / \mathrm{min}$. 

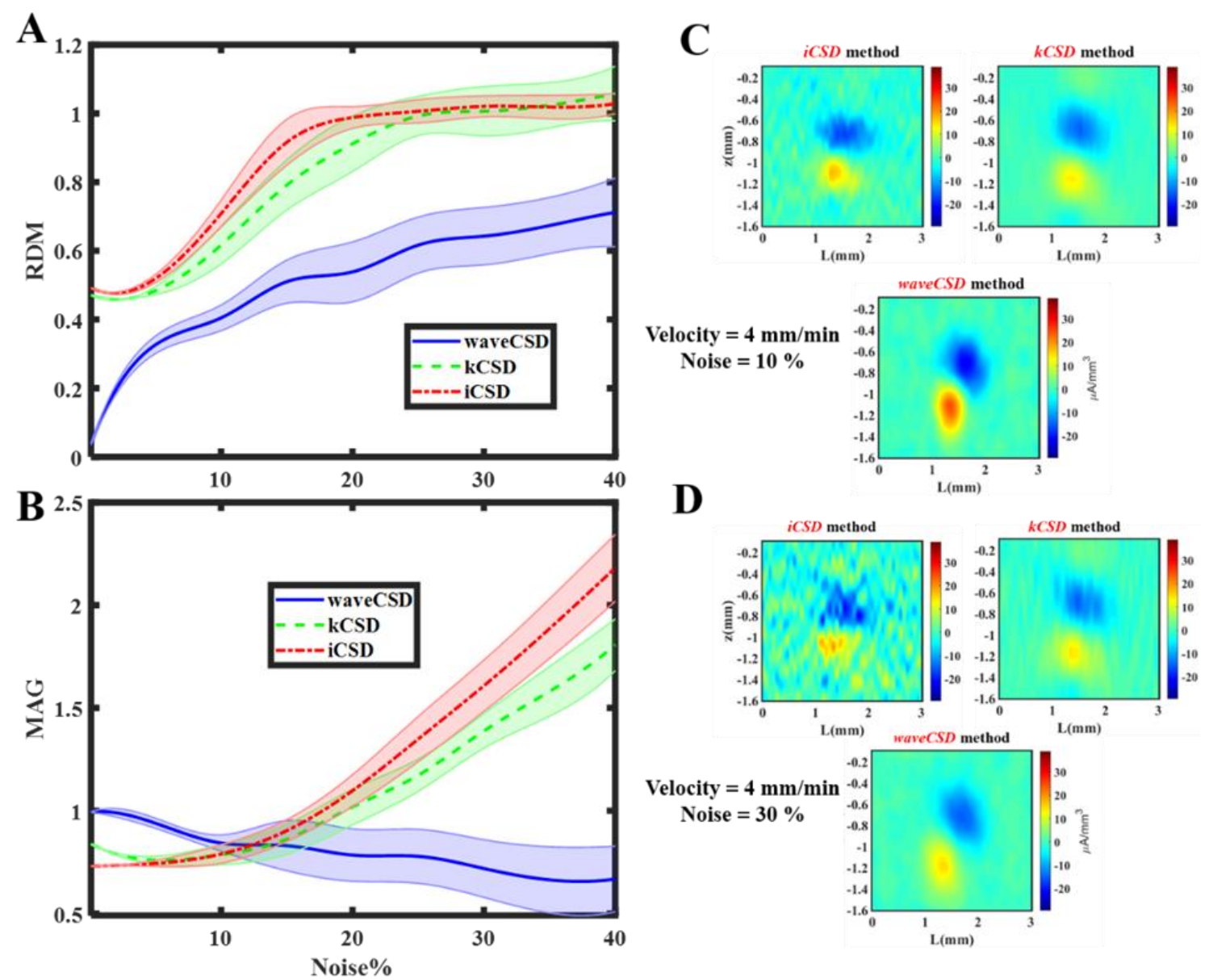

D
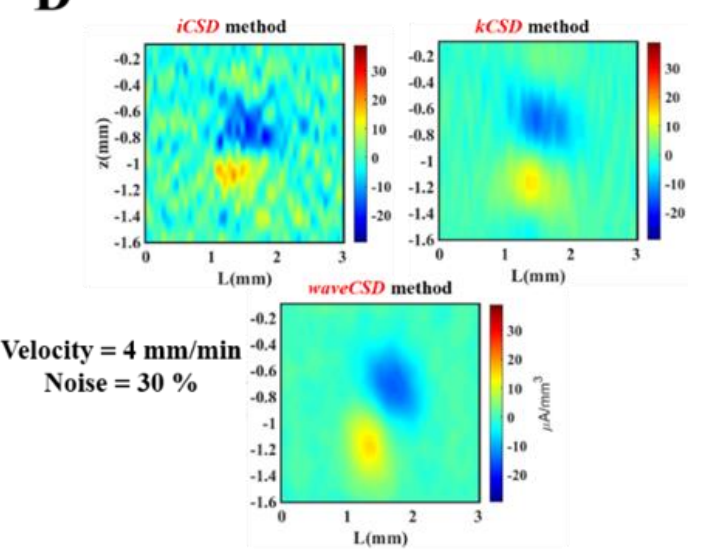

Figure 4-2Effect of observation noise on reconstruction accuracy of waveCSD compared to iCSD and kCSD. Accuracy of reconstruction of all methods are compared for different values of Gaussian observation noise (as percent of the maximum LFP signal) added to the LFP data shown in Figure 4-1, top right panel. A) RDM values for all three methods across 50 realizations of the observations are shown as mean \pm std for each noise level. Noise reduction in the iCSD method was performed by applying a Gaussian spatial filter on the reconstructed CSD from unfiltered LFP recordings (Gaussian filter sigma: $\sigma_{\mathrm{gf}}=0.1[\mathrm{~mm}]$ ) Results indicate that at all noise levels waveCSD method outperforms iCSD and kCSD methods (RDM values closer to zero in wave CSD). B) MAG values for all three methods across 50 realizations of the observations are shown as mean \pm std for each noise level. Results show a decreasing trend in the MAG measure for waveCSD method as the noise level increases, while both iCSD and kCSD methods show the opposite trend. Two examples of the average reconstructed CSD profile using all three methods are provided in panels C and D for 10 and $30 \%$ noise levels, respectively. Parameters are as stated in Figure 4-1.

Figure 4-3 shows the comparison of the accuracy of the methods in CSD reconstruction at a range of known velocity values (between 3 to $8 \mathrm{~mm} / \mathrm{min}$ ) at $10 \%$ noise level. For the waveCSD method, the $\mathbf{Q}$ matrix corresponding to each known velocity was computed and the CSD was reconstructed. For each velocity value, optimal parameters for kCSD and 
iCSD models were selected as described in Section 4.2.3. The MAG measure remains almost unchanged with wave velocity in all methods (Figure 4-3 A). The RDM measure exhibits a similar behavior in KCSD and iCSD methods, while it slightly increases with velocity in the waveCSD method (Figure 4-3 B). For the given waveform, waveCSD shows better performance in both RDM and MAG measures compared to kCSD and iCSD. Examples provided in panels $\mathrm{C}$ and $\mathrm{D}$ show source reconstruction for each method at 4 and $7 \mathrm{~mm} / \mathrm{min}$, respectively.
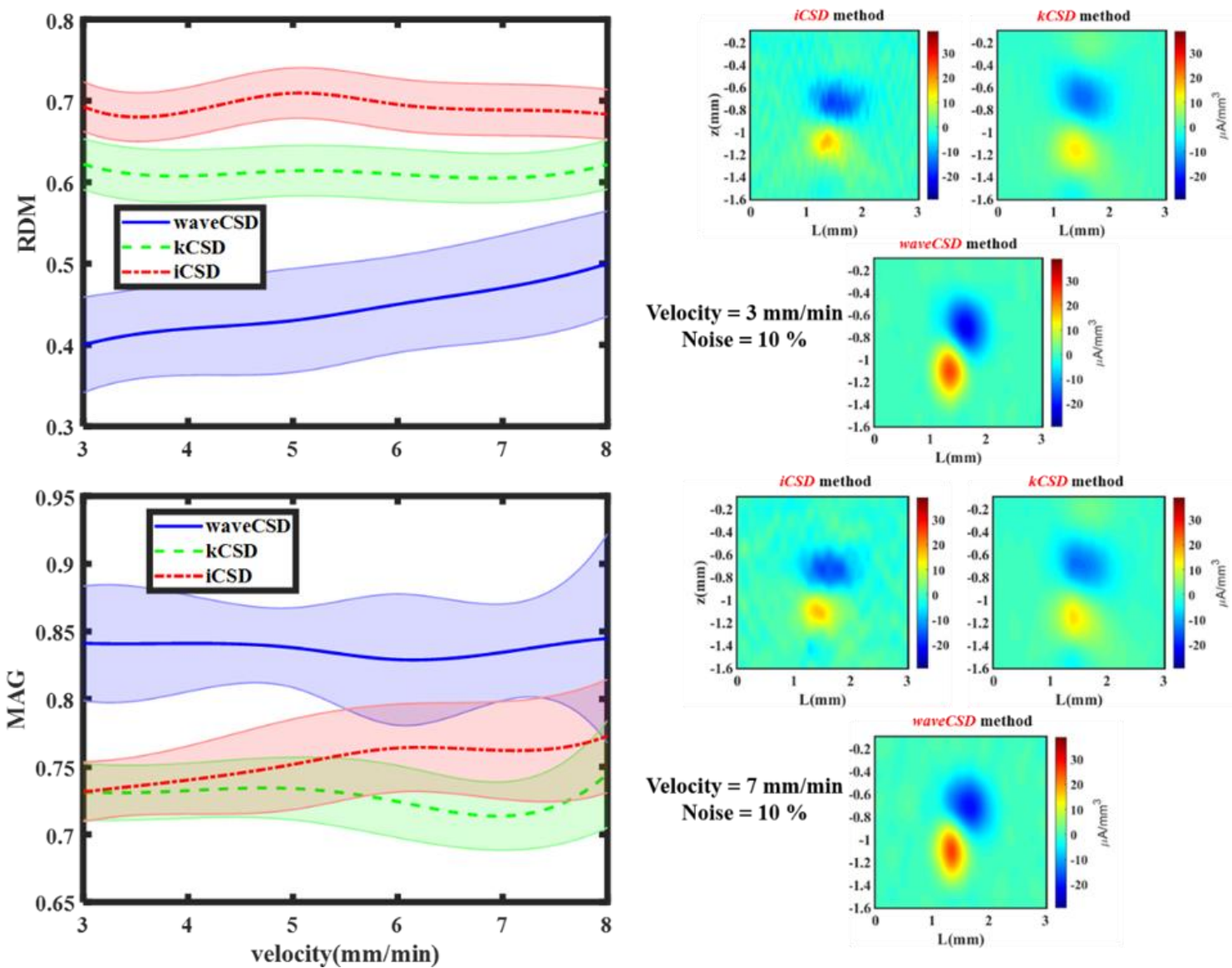

Figure 4-3 Effect of waveform velocity on the reconstruction accuracy of waveCSD, kCSD and iCSD methods. The accuracy of methods, as measured by RDM and MAG, are compared as a function of the known velocity of the ground-truth waveform presented in Figure 1 A, top left panel. 10\% Gaussian noise was added to the LFP data, and optimal parameters of kCSD and iCSD methods were selected for each velocity value as explained in Section 4.2.3. RDM and MAG values are shown as mean \pm std across 50 realizations of the observation noise. Noise reduction in the iCSD method was performed by applying a Gaussian spatial filter on the reconstructed CSD from unfiltered LFP recordings (Gaussian filter sigma: $\sigma_{\text {gf }}$ 
$=0.1[\mathrm{~mm}])$. A) $\mathrm{kCSD}$ and $\mathrm{iCSD}$ show no change in the RDM value at different velocities, while waveCSD RDM increases slightly with the increase in wave velocity. B) All MAG traces remain almost unchanged across different velocity values. Two examples of the average reconstructed CSD profile using all three methods are provided in panels $\mathrm{C}$ and $\mathrm{D}$ for 3 and $7 \mathrm{~mm} / \mathrm{min}$ wave velocity, respectively. Parameters are as stated in Figure 4-1.

As mentioned earlier, in simulations shown in Figure 4-1 to Figure 4-3, the parameter $d$ in iCSD method was selected based on the optimal radius $(\mathrm{R})$ of $\mathrm{kCSD}$, obtained from k-fold cross-validation technique. Since model parameter selection algorithms are not implemented for iCSD, in Figure 4-4 we demonstrate the changes in RDM and MAG of iCSD method as a function of different values of $d$. For comparison purposes, we also show the constant traces of these measures for waveCSD - by definition independent from $d$. For iCSD both measures decrease with the diameter increase, relatively faster for MAG than for RDM (RDM from $\sim 0.85$ to $\sim 0.8$, MAG from $\sim 4.5$ to $\sim 1.4$, for $d$ values from 300 to $700 \mu \mathrm{m}$ ), a trend that persisted for even extremely large values of $d$ (as RDM $\rightarrow 0.77$ and MAG $\rightarrow 0.70$ for $d \rightarrow+\infty)$. In this limit, in spite of MAG measure indicating a similar performance of both methods, waveCSD still outperforms iCSD with regards to RDM. 


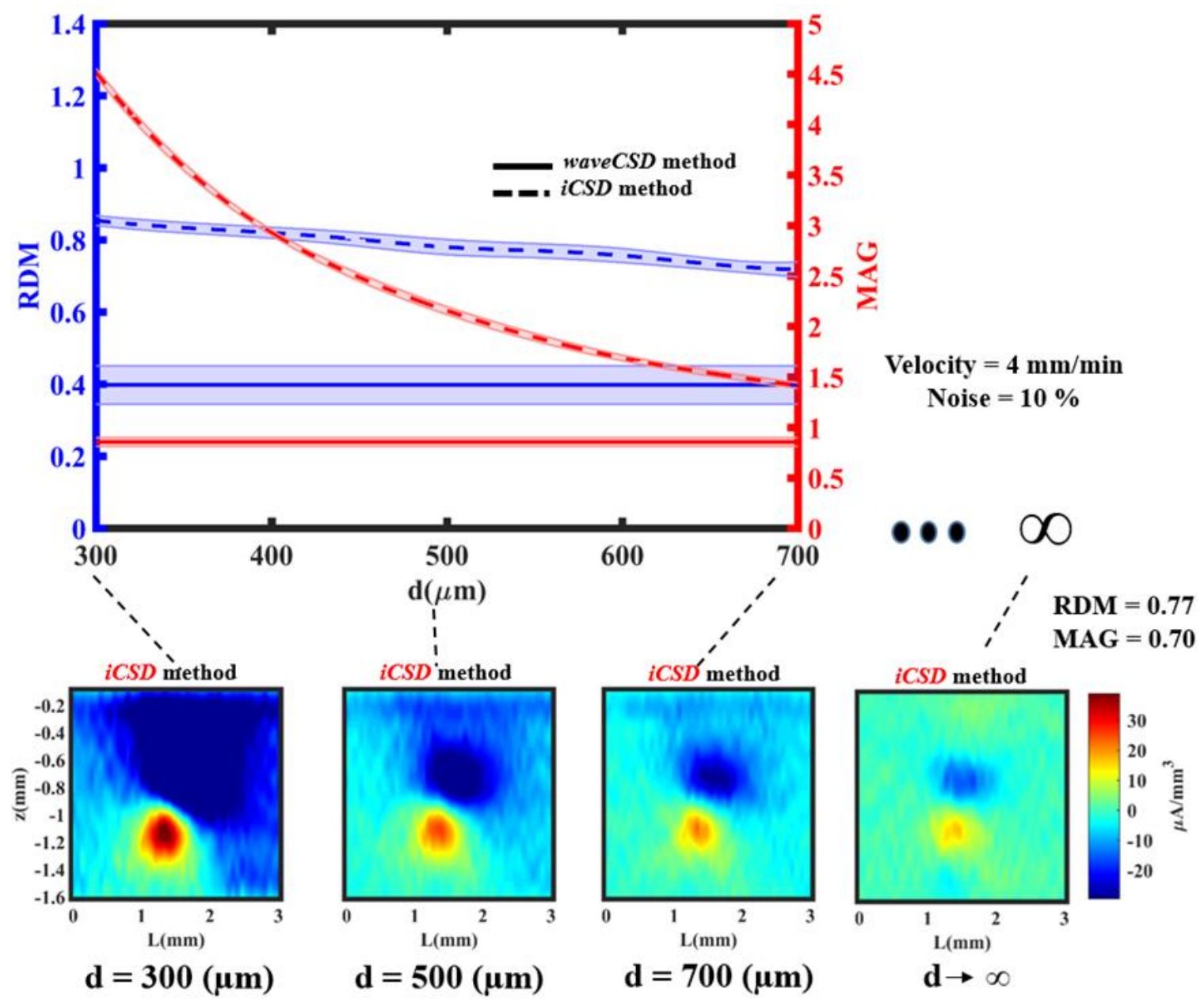

Figure 4-4 Dependency of CSD reconstruction accuracy to the diameter of the designated cylinder used in the iCSD method. The top panel shows that both RDM and MAG values in the iCSD method decrease as a function of $\mathrm{d}$. However, the changes are more drastic for the MAG measure (MAG: $\sim 4.5 \rightarrow \sim 1.5$; RDM: $\sim 0.85 \rightarrow \sim 0.75$, for $\mathrm{d}: 300 \rightarrow 700 \mu \mathrm{m}$ ). A limit case of $\mathrm{d} \rightarrow \infty$ is also provided in the bottom panel. Noise reduction in the iCSD method was performed by applying a Gaussian spatial filter on the reconstructed CSD from unfiltered LFP recordings (Gaussian filter sigma: $\sigma_{\mathrm{gf}}=0.1[\mathrm{~mm}]$ ). waveCSD method is independent of $\mathrm{d}$, thereby both measures are constant throughout. $\mathrm{v}=4 \mathrm{~mm} / \mathrm{min}$, and $10 \%$ noise is added to the LFP data. The rest of the parameters are as stated in Figure 4-1.

\subsubsection{Robustness of the waveCSD method to noise and MEA resolution}

Figure 4-5 shows the robustness of the reconstructed CSD profile using the waveCSD method to the presence of noise and to the number of electrodes used in the probe. For each noise level, electrode number and velocity value, the average of MAG and RDM across 50 CSD reconstruction trials is shown. Figure 4-5 $\mathrm{A}$ and 5B show the accuracy of reconstruction at different ranges of propagation velocity and varying noise levels. Figure 
4-5 A and Figure 4-5 B show that at any known velocity value, increasing the noise level reduces the accuracy of the source reconstruction (RDM away from 0 , and MAG away from 1). Similarly, at any given noise level, increasing the velocity of the waveform decreases the reconstruction accuracy. Figure 4-5 C and Figure 4-5 D demonstrate a case in which a constant velocity of $4 \mathrm{~mm} / \mathrm{min}$ is used for all simulations, and the source reconstruction accuracy is analyzed with respect to the number of electrodes in the probe. As can be observed in both panels, at any electrode resolution, increasing the noise level increases the error of reconstruction. Meanwhile, at any given noise percentage, increasing the resolution of the recording results in increased reconstruction accuracy.

A

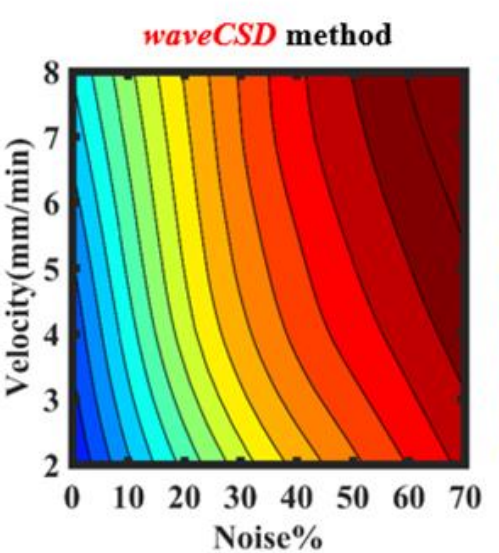

C

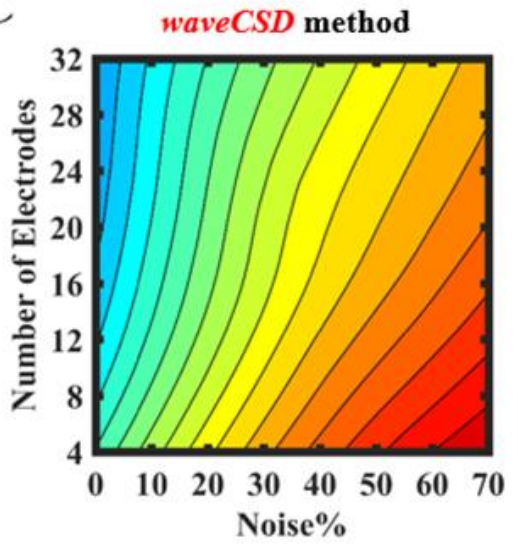

Velocity $=4 \mathrm{~mm} / \mathrm{min}$
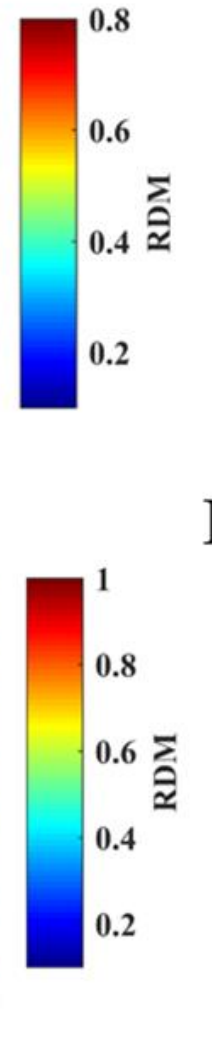

D

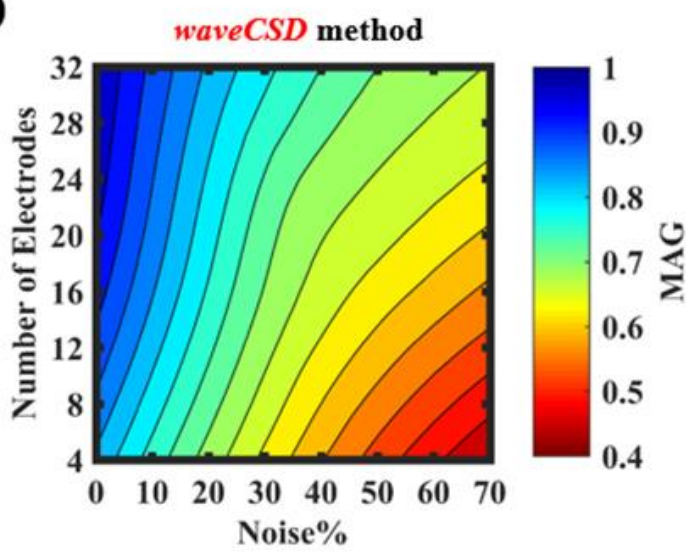

Velocity $=4 \mathrm{~mm} / \mathrm{min}$ 
Figure 4-5 Robustness of the CSD analysis using waveCSD method to noise and electrode resolution. A-B) RDM and MAG measures are shown for $\mathbf{c}_{\text {wave CSD }}$ from the LFP data in Figure 4-1 A right panel, for a range of known velocities and different noise levels. The colorbar for the MAG measure is inverted compared to RDM (blue colored regions indicate desirable RDM and MAG values; red regions indicate areas with high error in reconstruction). At any given velocity, increase in the noise level decreases the accuracy of the reconstruction (RDM away from zero, and MAG away from one) and vice versa. C-D) RDM and MAG measures are shown as a function of electrode resolution at different noise levels for the average reconstructed CSD profile ( $\mathbf{c}_{\text {waveCSD }}$ ) of the LFP data of the ground-truth waveform. At any electrode resolution, increase in the noise level decreases the accuracy of reconstruction, whereas at any given noise, increasing the number of recording electrodes, increases the reconstruction accuracy. Parameters are as described in Figure 4-1 except $\mathrm{n}_{\tau}=30$ and $\mathrm{n}_{\mathrm{z}}=10$.

\subsubsection{Errors associated with uncertainties in the knowledge of wave velocity in waveCSD method}

So far, we have assumed that one has an accurate knowledge of the velocity of the propagating wave. However, the velocity measurement might not be highly accurate. We performed the following procedure to evaluate the effect of assuming incorrect values of the velocities in the wave CSD reconstruction. The ground-truth CSD waveform (as shown in Figure 4-6 A, left panel) was assumed to propagate with a velocity of $5 \mathrm{~mm} / \mathrm{min}$. The resultant LFPs (shown in Figure 4-6 A, right panel) was calculated using Equation 4-6 with $10 \%$ noise. Then, a set of CSDs (Figure 4-6 B) were estimated using $\mathbf{Q}$ matrices corresponding to inaccurate velocities ranging from 4 to $6 \mathrm{~mm} / \mathrm{min}$. As observed in panel $\mathrm{B}$, the most noticeable difference in the reconstructed profiles is the change in the location of the CSDs predominantly in the $x$ direction. This observation is verified when RDM and MAG measures are compared before and after a linear co-registration of the reconstructed profiles through translation along the $x$ axis (Figure 4-6 C and Figure 4-6 D, respectively). Results in both Figure 4-6 C and Figure 4-6 D show that if reconstruction is performed using the $\mathbf{Q}$ matrix with an assumed accurate velocity (Figure 4-6 B, middle box), the reconstruction error is minimal (Notice the RDM in Figure 4-6 C). Figure 4-6 C also shows that while the RDM measure is significantly affected by estimations assuming inaccurate 
velocities; the MAG measure does not significantly change. These results indicate that in spite of lack of precise knowledge of the velocity of the propagating wave, the waveCSD method can still provide CSD reconstruction with high accuracy, given the consideration that the location of the CSDs might be shifted mainly in the $x$ direction.

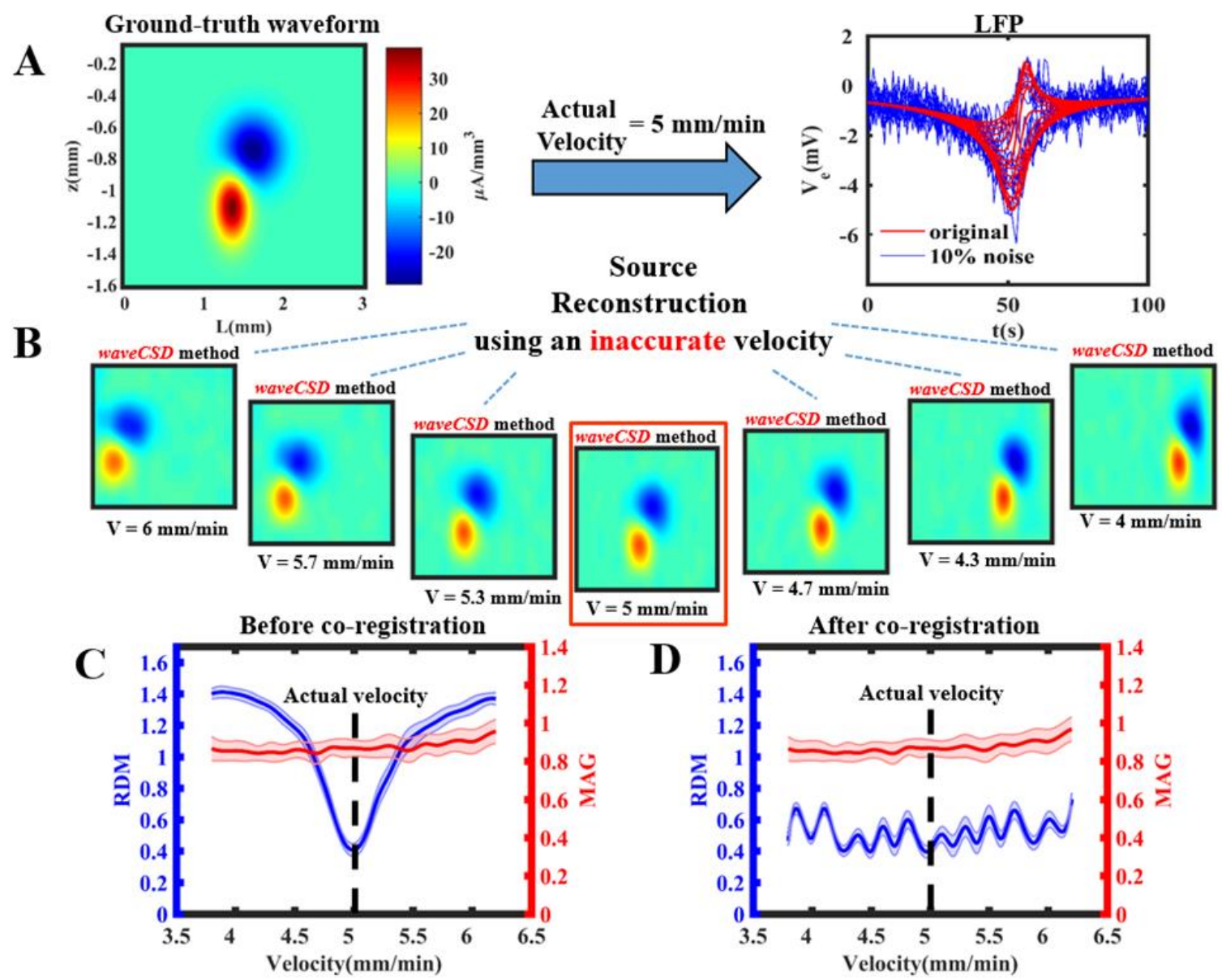

Figure 4-6 Reconstruction accuracy under uncertainties in the knowledge of velocity of the waveform in the wave CSD method. A) Under 10\% noise, LFPs (right panel, red traces: unnoisy, blue traces: noisy) from an A1x16 probe were simulated assuming a ground-truth CSD waveform (left panel) propagating with at $\mathrm{V}=5$ $\mathrm{mm} / \mathrm{min}$ towards the electrodes. B) The noisy simulated LFPs were used to estimate the CSD using the waveCSD method assuming a range of wave velocities from 4 to $6 \mathrm{~mm} / \mathrm{min}$. Profiles show a monotonic shift in the position of CSDs relative to the error in the assumed velocity. C) RDM and MAG measures for the reconstructed profiles using velocity values are shown as mean \pm std. If the actual velocity is used for the reconstruction (highlighted box in panel B), the accuracy is maximal. Note that the RDM measure is significantly dependent on the velocity error, while the MAG measure does not significantly change. D) Both RDM and MAG are almost unchanged after linear coregistration of reconstructed profiles in panel $\mathrm{B}$ along the $x$ axis. Parameters used in simulations: temporal resolution of the $\mathbf{Q}$ matrices: $1[\mathrm{~s}] ; \mathrm{n}_{\tau}=20 ; \mathrm{n}_{\mathrm{z}}=10$. Rest of the parameters are as described in Figure 4-1. 


\subsubsection{Parameter estimation in wave CSD method using GCV from the LFP data}

As explained in Section 4.2.1, the main parameters of the waveCSD method, i.e., $\alpha$ and $v$, are estimated from the LFP data using generalized cross-validation technique. To test the accuracy of parameter estimation, a ground-truth CSD waveform shown in Figure 4-7A left panel is assumed to be generated $6 \mathrm{~mm}$ away from an A1x16 probe and propagate towards the electrodes at $5 \mathrm{~mm} / \mathrm{min}$. The actual $\alpha$ used for the generation of LFPs (Figure 4-7 A right panel) was set to $3 \mathrm{~mm}$. The waveform is generated by superposition of 10 Gaussian profiles with random amplitude, spread, and angle using the methodology described in [153]. A wide range of $\alpha$ and $v$ values were selected $(\alpha \in[1,5] ; v \in[3,7])$, and for each pair of parameter values, the corresponding Q matrix was calculated. Using these matrices, source reconstruction was performed on the LFP data without observation noise, and the pair which minimized the GCV score was selected as the optimal parameter set (red dot in Figure 4-7 B panels). As can be observed in the figure, the method successfully found actual parameter values in the absence of noise. Moreover, the optimal parameter set also coincided with optimal RDM and MAG measures. The same procedure was repeated for a wide range of noise levels in the LFP data (Figure 4-7 C). For each percentage of noise and for each parameter pair, 50 realizations of the noisy LFP were reconstructed. Figure 4-7 C confirms the successful parameter estimation even at high levels of observation noise in the LFP data. 

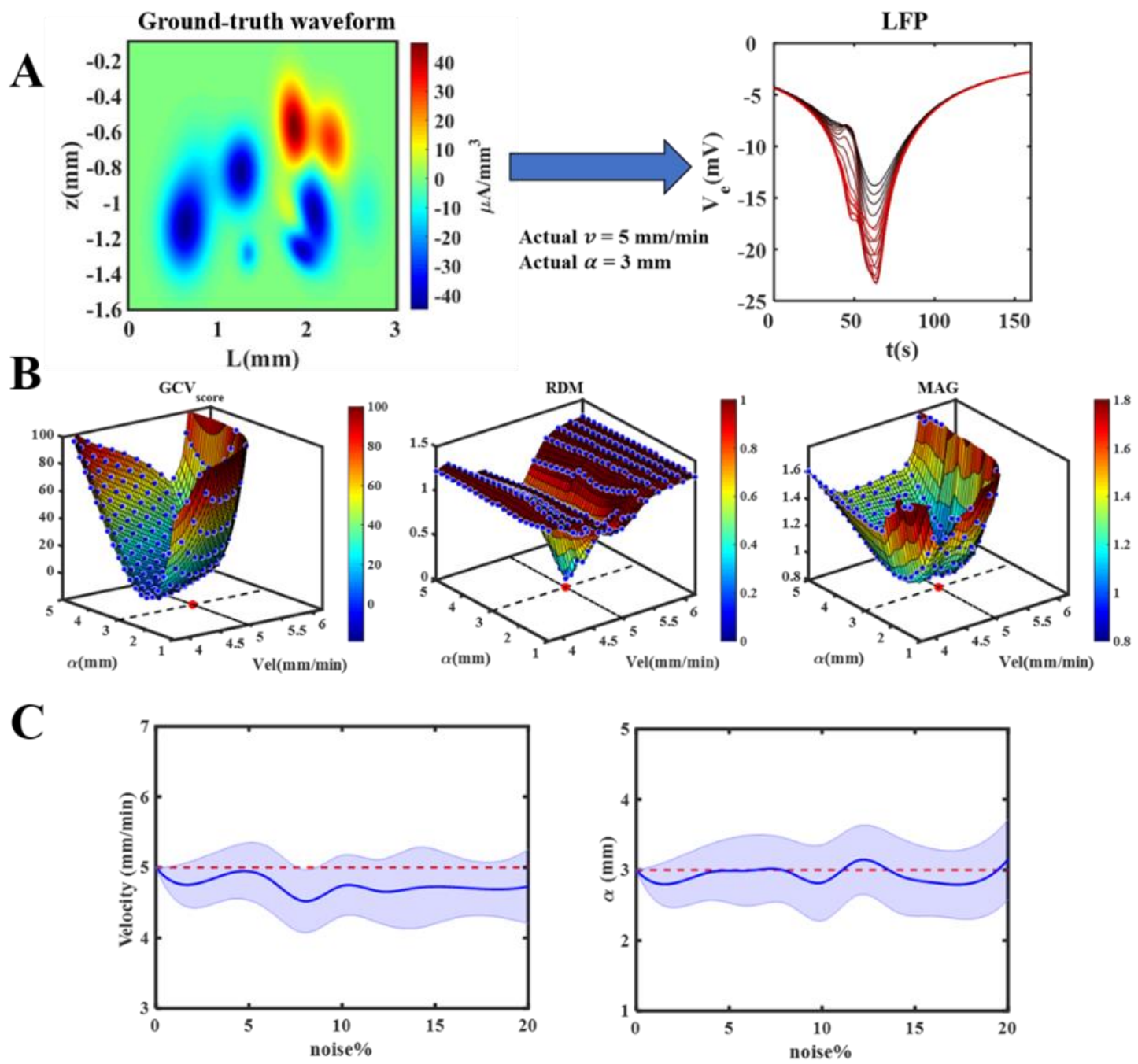

Figure 4-7 waveCSD model parameter estimation from the LFP data using GCV technique. A) A groundtruth profile was generated via superposition of 10 Gaussian profiles with random amplitude, spread, and angle (left panel). The resultant LFP profile (right panel) were generated from a A1x16 electrode assuming the waveform was generated $6 \mathrm{~mm}$ away from the electrodes and propagated towards the probe with a velocity of $5 \mathrm{~mm} / \mathrm{min}$ (black $\rightarrow$ most superficial electrode, and red $\rightarrow$ deepest electrode in the probe). The limits of the cortex in the $y$ direction (i.e. $\mathrm{y}_{\mathrm{f}}=-\mathrm{y}_{\mathrm{s}}=\alpha$ ) was set to be from $[-3,3][\mathrm{mm}]$. B) For a wide range of velocity and $\alpha$ values the corresponding $\mathrm{Q}$ matrices were calculated (blue dots) and the parameter pair which minimized the GCV score was selected as the optimal set (the red dot). RDM and MAG measures are also shown for each parameter pair. In all simulations in the panel no noise was added to the LFP data. C) Parameter estimation was performed in the presence of varying percentages of the observation noise in the LFP data. For each noise level and each parameter set, 50 realizations of the noisy data were reconstructed. Data is shown is mean \pm std, and the actual values of velocity and $\alpha$ are shown in red dashed lines.

\subsubsection{Transmembrane current sources during cortical spreading depression}

LFP recordings from five rats were used to reconstruct the transmembrane CSDs during cortical spreading depression. Figure 4-8 A shows the LFP data from one rat recorded using 
an A1x32 probe. To solely account for the distribution of CSDs during a cortical spreading depression event, CSD analysis was performed after removing the natural resting-state brain activity and the silencing period (i.e. regions before and after the highlighted box in Figure 4-8 A). LFP data was down-sampled to the temporal resolution of the $\mathbf{Q}$ matrix used for the reconstruction (Figure 4-8 A inset). The reconstructed CSD profile of Figure 4-8 A inset is shown in Figure 4-8 B. The corresponding $\mathbf{Q}$ matrix for the reconstruction was computed based on an assumed velocity of $3 \mathrm{~mm} / \mathrm{min}$, a value commonly reported in literature for the velocity of the moving wave during cortical spreading depression $[175$, $187,188]$. Note that we have already addressed the potential errors that are introduced in the reconstruction if this choice of velocity is not accurate (see Figure 4-6).
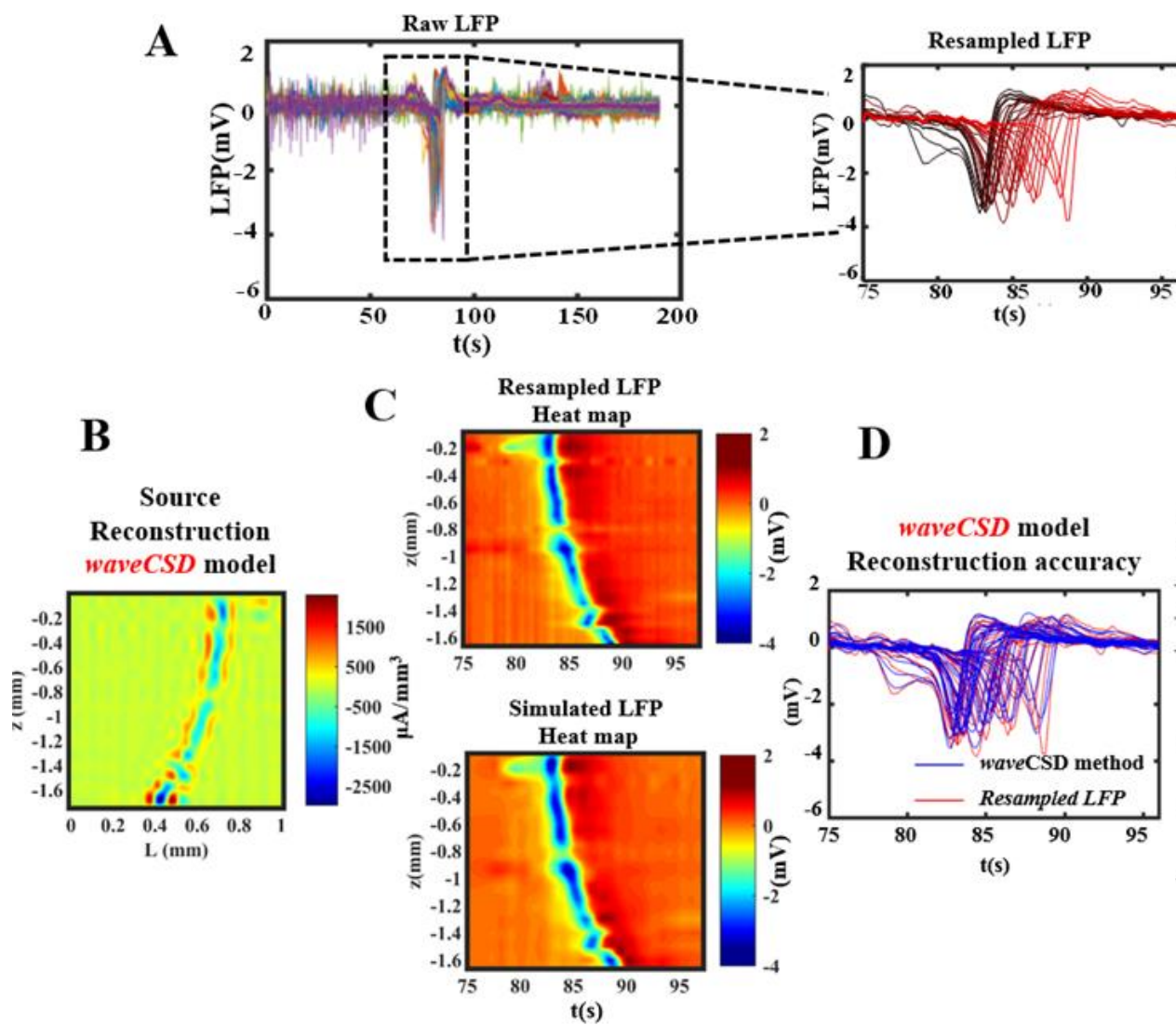

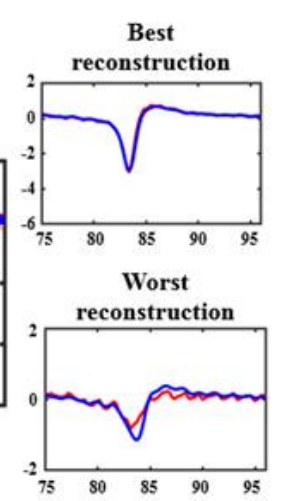

$t(s)$

Figure 4-8 CSD analysis of LFPs recorded during cortical spreading depression in rats using the waveCSD method. A) The LFP recordings of one rat using an A1x32 probe during cortical spreading depression is 
shown in the left panel. The resting brain activity and silencing period after the event were excluded in the source reconstruction using the waveCSD method (right panel). LFP data was resampled to the temporal resolution of the $\mathbf{Q}$ matrix used for the CSD analysis (i.e., 0.2 [s]). The resampled data is shown using a color gradient from black to red (black $\rightarrow$ most superficial electrode, and red $\rightarrow$ deepest electrode in the probe). B) Reconstructed CSD profile of the resampled LFP data in panel A inset using the waveCSD method. C) Heat maps of the resampled LFP and the simulated LFP (resulting from forward modeling of the profile in the panel B using Equation 4-8) are demonstrated. Please note that panels B and C have different $\mathrm{x}$-axes. For the reconstructed waveCSD model (panel $\mathrm{B}$ ), the $\mathrm{x}$-axis represents space, along the propagating direction. For the LFP heat maps (panel C), the $x$-axis represents time. D) Simulated LFP is superimposed onto the original resampled LFP. The insets demonstrate the electrodes with the best and the worst reconstruction determined based on the sum of square of the errors between the original and the reconstructed trace. Parameters used for the computation of the $\mathbf{Q}$ matrix for CSD analysis of the data: $\mathrm{n}_{\tau}=30 ; \mathrm{n}_{\mathrm{z}}=20 ; \mathrm{L}=1[\mathrm{~mm}]$; temporal resolutions: 0.2 [s]. The rest of parameters are as described in Figure 4-1.

Figure 4-8 D shows the extent to which the data (Figure 4-8 A inset) can be fitted by the forward model in Equation 4-8 using the estimated CSD obtained in Figure 4-8 B. To that end, the "simulated" LFP was computed from the estimated CSD and was superimposed onto the original LFP. Figure 4-8 D insets show the electrodes with best and worst LFP reconstruction, as determined by the sum of square of errors between the original LFP trace and the reconstructed trace. The LFP heat maps, i.e., the temporal voltage profile, of the resampled LFP in Figure 4-8 A inset and the simulated LFP are compared in Figure 4-8 C.

Figure 4-8 A demonstrates the grand average CSD profile across rats after nonlinear diffeomorphic coregistration of the reconstructed CSDs (data from five rats; see Section 4.2.4). The average CSD profile suggests a complex, multipolar configuration of current sources in different layers of the cortex during cortical spreading depression. The accurate probe positioning and penetration depth were verified via histology, postmortem (Figure 4-9 B). The schematic in Figure 4-9 C illustrates major layer-specific cellular populations in the neocortex. 


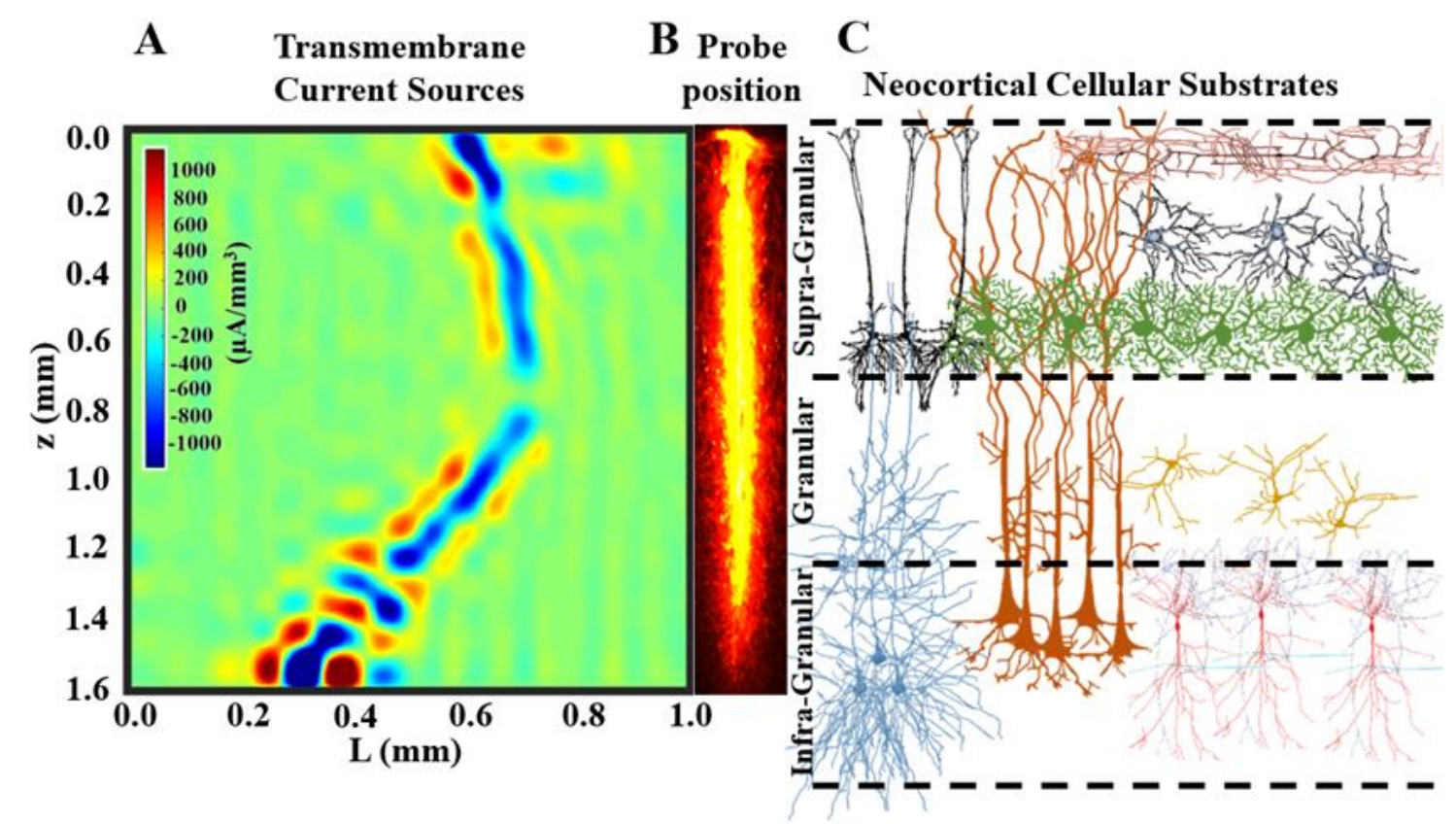

Figure 4-9 Average profile of transmembrane current sources during cortical spreading depression in rats after nonlinear coregistration of the reconstructed profiles. A) The grand average transmembrane CSD profile across five rats during cortical spreading depression using the waveCSD method is depicted. The averaging was performed after application of a landmark-based nonlinear diffeomorphic coregistration to individual reconstructed current sources. B) The histology image showing the accurate positioning and the desired penetration depth of the probe in the rat neocortex. C) The schematic representing major cellular substrates across different layers (i.e., Supra-Granular, Granular, and Infra-Granular) of the rat neocortex, e.g. excitatory pyramidal cells (layer II/III - black, layer V - brown and layer VI - blue), excitatory spiny stellate cells (yellow), inhibitory interneurons (basket cells - blue/black and Martinotti cells - red/blue), and layer II/III glia cells (green).

\subsection{DISCUSSION}

CSD analysis of electrical signals in the brain has been revolutionized owing to the progress in development of high resolution MEAs for the spatiotemporal signal acquisition in different experimental settings. To date, CSD analysis methods utilizing LFP recordings from linear probes do not take the propagation of neuronal activity along the cortical sheet into account. In this study, we provide a theoretical framework for the reconstruction of propagating CSDs with the assumption that these waves are planar and have a constant velocity. An immediate application of the waveCSD method is the CSD analysis of transmembrane currents during a cortical spreading depression event. 


\subsubsection{Comparison of wave CSD, $\mathrm{kCSD}$, and $\mathrm{iCSD}$ methods in the CSD analysis of propagating neuronal activity}

The iCSD method developed by Pettersen et al. [161] has shown high level of accuracy in a variety of applications and has been one of the most widely-used CSD analysis techniques in the past decade $[152,161,162]$. The development of $\mathrm{kCSD}$ method, which provided a kernel-based methodology, further overcame main deficiencies of the iCSD method through incorporation of regularizing techniques to improve reconstruction quality, as well as inclusion of cross-validation algorithms to estimate optimal parameters given a data set. However, the assumptions in these methods do not account for propagation of neuronal activity along the neocortex in CSD reconstruction. Thus, if these methods are used for CSD analysis of conditions where such propagations are involved, the interpretations have to be drawn with care and results might not necessarily be highly accurate. It should be noted that in this chapter, iCSD is synonymously referred to spline-iCSD method for laminar probes described in [161], and not the inverse current source density analysis method in general. In the case of CSD analysis of a propagating neocortical wave, the following key assumptions of the iCSD and kCSD methods are violated:

Firstly, when the wave of neuronal activity approaches the probe, current sources far from the electrodes contribute to the LFP data. Hence, the assumption that only current sources near the electrodes, i.e. inside the designated cylinder, contribute to the LFP data is not valid. Consequently, the choice of $d$ in the analysis of CSDs emerging from the propagation of neuronal activity using the iCSD or kCSD methods can significantly affect the accuracy of the reconstruction. In particular, the lower the value of $d$, the higher the magnitude of reconstructed CSDs to account for the contribution of far current sources (see 
Figure 4-4 for the iCSD method). As shown in Figure 4-1, Figure C4- 1 and Figure C4- 2, the cross-validation algorithm in kCSD method selected significantly higher diameter of current sources around the probe compared to the typical value of a cortical column in the barrel cortex $(\sim 500[\mu \mathrm{m}])$, primarily used in the iCSD method for reconstruction of the sources. Even though these choices of parameters improved the reconstruction accuracy (compare Figure 4-1 and Figure 4-4); still, the reconstructed CSD profiles are far from the ground-truth waveforms (Figure 4-1, Figure C4- 1 and Figure C4- 2). This indicates that if model assumptions are not in line with the physiology of the phenomenon, e.g., a planer wave in this study, the reconstruction error can be significant.

It is also noteworthy that several studies [189-194] cast doubt on the traditional assumption that LFPs are generated within hundreds of micrometers from the recording electrode $[190$, 195, 196], by providing evidence on the spatial reach of the LFPs in the order of several millimeters. Additionally, the contribution of current sources to the LFP data is highly dependent on correlation. Thus, in physiological phenomena where the spatial activity is highly correlated, distant sources do indeed contribute to the LFP data [194], which is surely the case for cortical spreading depression.

Secondly, in the case of a propagating neocortical wave of neuronal activity towards the linear probe, CSDs cannot be assumed constant in the tangential direction, i.e. there is no tangential symmetry of the current sources around the electrodes. Both iCSD and kCSD methods provide the distribution of CSDs as though they were tangentially symmetric around the probe within the designated cylinder, which does not describe the actual phenomenon. 
Since the assumptions in the derivation of waveCSD method are particular to phenomena involving a planar wave of neuronal activity, the accuracy of the reconstruction is higher than those of kCSD and iCSD methods in simulations performed in this chapter (see Figure 4-1 to Figure 4-4, Figure C4- 1 and Figure C4- 2). Hence, even though the application of $\mathrm{kCSD}$ or iCSD methods provides valuable information about the distribution of CSDs in a variety of experimental paradigms, they must be cautiously used and interpreted if they are used to analyze currents originating from propagating neuronal activity. It should be noted that the same reasoning is applied to waveCSD method, where one should expect lower accuracy compared to kCSD or iCSD methods if waveCSD is applied for experimental paradigms where neuronal activity is static and of cylindrical symmetry. Similarly, waveCSD method is expected to have high reconstruction errors if the propagating wave does not have the same characteristics (e.g., constant velocity and planar symmetry) as ones assumed in model derivation. Hence, the applicability of waveCSD has to be evaluated for each particular experimental situation. The waveCSD method, as it stands, is expected to yield higher reconstruction accuracy when applied to global brain traveling waves, e.g., those related to brain rhythms [167, 169-171, 173], sensory-evoked activity or spreading depression waves, as compared to more local spiral waves in the brain [197].

\subsubsection{CSD analysis of cortical spreading depression}

In an early study, Wadman et al. [198] performed a CSD analysis of the sustained DC shifts attributed to spreading depression in CA1 and dentate gyrus of the rat hippocampus using the traditional CSD analysis method [159]. The spreading depression was induced by repetitive electric stimulation of different afferent pathways. The results of the study 
indicated a large current sink in the proximal regions of the apical dendrites and major current sources in the deep layers where the cell soma resides. Authors reported changes in the polarity of CSDs both with time and along the depth of hippocampus. Another study by [199] also used the traditional CSD analysis method to obtain current source densities in CA1 of rats during spreading depression induced by electrical stimulation of hippocampal ipsilateral CA3 region. The temporal CSD profile of spreading depression showed a rapid (>0.5 s), high intensity, current source and sink sequence at the front of the wave. More recently, using the same CSD analysis methodology, [200] observed similar intense, rapid outward-inward current sequences at the wavefront, followed by more spread current sources and sinks, in $\mathrm{KCl}$-evoked spreading depression in the barrel cortex of rats.

The temporal and spatial changes in the polarity of CSDs were also observed in our study despite differences in the brain region of interest, spreading depression induction method, and the CSD analysis methodology (see Figure 4-8 A and Figure 4-9 A). The average profile of the transmembrane currents during cortical spreading depression (Figure 4-9 A) indicates that the overall shape of the reconstructed CSD is consistent across all the rats used in this experiment. The magnitude of currents appears to be significantly larger at deeper layers (Infra-Granular). Additionally, the change in the polarity of CSDs is more drastic in deeper layers (Infra-Granular) in comparison with superficial (Supra-Granular) and middle layers (Granular).

Under the set of assumptions used in the derivation of the waveCSD method (see Section 4.2.1), the first current sources which emerge during a cortical spreading depression event 
appear to be at the Supra-Granular layer. Subsequently, the transmembrane currents seem to originate in the Granular region and propagate towards the Supra-Granular and InfraGranular layers. This can be viewed as the noticeable shift in the relative position of the CSDs across the depth of the cortex in Figure 4-9 A. The knowledge of distribution of the transmembrane currents during a cortical spreading depression event can provide important insights into understanding mechanisms underlying this complex phenomenon. Detailed analysis of such mechanisms, however, is beyond the scope of the present study.

\subsubsection{Improvement in the wave CSD method and future directions}

The waveCSD method assumes that the propagation velocity of the wave of neuronal activity is constant across all layers of the neocortex. This assumption is not valid in all experimental paradigms. In cortical spreading depression, for instance, Basarsky et al. [201] reported that the propagation of the depolarization wave is significantly faster in the outmost layers of rat cortical slices. In another study, Richter and Lehmenkuhler [202] provided evidence for the existence of a barrier for the genesis and propagation of spreading depression along the depth of the cortex. These results were in agreement with early studies by Leão and Morison [203] and Leão [204] indicating that cortical spreading depression genesis and duration is layer sensitive. A recent study by our group (unpublished data) also revealed that the propagation velocity of cortical spreading depression in rats changes along the depth of the cortex.

All these evidence, along with the variability in the reported propagation velocity of the cortical spreading depression in previous studies [175], indicate that an avenue for 
improvement in the waveCSD method is the incorporation of depth-dependency in the tangential propagation velocity (i.e. $v$ as a function of $z$ ).

The observations presented in Figure 4-6 corroborate the idea that the mismatch in the actual versus accounted velocity value in the waveCSD method results in a shift in the position of the reconstructed CSDs, predominantly in the $x$ direction. Thus, the use of a constant velocity for CSD analysis of a cortical spreading depression event might explain relative positioning of CSDs across cortical layers as shown in Figure 4-8 and Figure 4-9.

The waveCSD method assumes the propagation of a single wave of neuronal activity towards the electrodes. Evidence in literature point to the co-existence of multiple distinct waves during spreading depression (e.g., calcium waves propagating along and across layers in hippocampal organ cultures [205], as well as in mouse neocortical slices [206]). Thus, a more general and realistic approach for the CSD analysis of such multi-mechanistic phenomena using the waveCSD method is to incorporate multiple waves approaching the electrodes along different cortical lamina. However, it is important to note that the waveCSD method is a general investigative tool which encompasses a variety of applications and is not restricted to cortical spreading depression. Hence, incorporation of the above-mentioned modifications was not the focus of the current study.

Similar to other methods for CSD analysis, waveCSD only takes the Ohmic currents into account in the reconstruction of CSDs from their respective LFP reflections. However, there has been a growing controversy in recent years on the potential role of diffusive currents and the extent of their importance on the recorded extracellular potentials [156, 
162, 207-210]. A recent theoretical study by Halnes and colleagues [211] suggests that diffusive currents can indeed have comparable influences on the extracellular potentials in the vicinity of a small population of active neurons in the cortex in the range of frequencies up to $10 \mathrm{~Hz}$. Additionally, theoretical research groups have also demonstrated that the magnitude of diffusive currents in both intra- and extra-cellular space can be comparable to Ohmic currents in the presence of steep concentration gradients in the ionic substrates [211, 212]. Cortical spreading depression is a clear-cut example of a neurological condition where such steep changes in the ionic concentrations is observed [213-216]. Hence, another future direction of the current research is to incorporate the diffusive currents in the derivation of the waveCSD method.

\subsection{CONCLUSIONS}

To our knowledge, the method outlined in this study is the first to account for the propagation of neocortical neuronal activity in the reconstruction of CSDs from their LFP reflections. Robustness of the method to the presence of observation noise, and the uncertainties in the knowledge of propagation velocity, was verified in different sets of simulations. Using simulations, the waveCSD method showed higher reconstruction accuracy compared to the iCSD method in the CSD analysis of transmembrane currents originating from propagating neuronal activity. In this study, we utilized the waveCSD method to provide a novel profile of the distribution of transmembrane CSDs during a cortical spreading depression event. As discussed earlier, the waveCSD method is far from ideal and several factors can enhance the reliability of the method in the analysis of CSDs in different experimental settings. However, since the waveCSD method is specifically 
designed for the CSD analysis of propagating neuronal waves in the cortex, we believe that the combination of this method with theoretical models as ones developed in [217], has the potential to provide a theoretical platform to unravel the underlying mechanisms of several neurological phenomena involving the propagation of neuronal activity.

\section{APPENDIX A4. DISCRETIZATION OF THE waveCSD FORWARD PROBLEM}

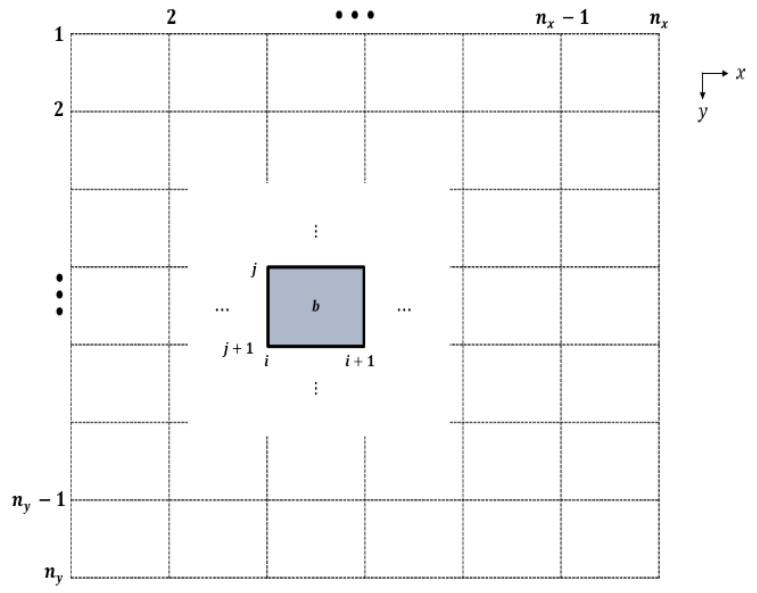

Figure A4- 1 Discretization of a rectangular grid of size nx x ny

Throughout this Appendix, with some modifications, we base our derivations on the methodology outlined in [152] and [154]. Before providing the detailed derivation of the discretized version of Equation 4-8 in the main document, we provide an introduction on bicubic spline in two dimensions.

Assume a rectangular grid shown in Figure A4- 1, where $i$ ranges from 1 to $n_{x}$, and $j$ ranges from 1 to $n_{y}\left(n_{x}\right.$ and $n_{y}$ are the number of nodes in the $x$ and $y$ directions, respectively). To interpolate $f(x, y)$ using bicubic splines in the rectangular grid shown in Figure A4- 1, we first recap the derivation of cubic splines in a single direction: 
Assume a set of $n$ data points $\left(x_{i}, f\left(x_{i}\right)\right)$ at regularly spaced grid points: $x_{1}<x_{2}<\cdots<$ $x_{n}$. The cubic spline $S(x)$ is defined as:

$$
S(x)=\left\{\begin{array}{cc}
S_{1}(x) & x_{1} \leq x \leq x_{2} \\
\vdots & \\
S_{i}(x) & x_{i} \leq x \leq x_{i+1} \\
\vdots & \\
S_{n}(x) & x_{n-1} \leq x \leq x_{n}
\end{array}\right.
$$

where $S(x) \in C^{2}$ in $\left[x_{1}, x_{n}\right]$ satisfying $S\left(x_{i}\right)=f\left(x_{i}\right)$, and each $S_{i}(x)$ is a cubic polynomial. For $x_{i} \leq x \leq x_{i+1}$, from [218] one can write:

$$
S_{i}(x)=P_{1}(x) f\left(x_{i}\right)+P_{2}(x) f\left(x_{i+1}\right)+P_{3}(x) f^{\prime \prime}\left(x_{i}\right)+P_{4}(x) f^{\prime \prime}\left(x_{i+1}\right)
$$

where

$$
\begin{aligned}
P_{1}(x)=\frac{x_{i+1}-x}{h_{x}} & ; P_{2}(x)=1-P_{1}(x) ; P_{3}(x)=\frac{1}{6}\left(P_{1}^{3}(x)-P_{1}(x)\right) h_{x}{ }^{2} ; P_{4}(x) \quad \text { Equation A4- 3 } \\
& =\frac{1}{6}\left(P_{2}^{3}(x)-P_{2}(x)\right) h_{x}{ }^{2}
\end{aligned}
$$

and $f^{\prime \prime}\left(x_{i}\right)=\sum_{k=1}^{n_{x}} G_{i, k}^{x} f\left(x_{k}\right)$

Equation A4- 4

$G^{x}$ is a square matrix of length $n$, whose matrix product with the vectorized function values at the nodes, will provide the second derivative estimation of $f(x)$ at the grid points. This matrix can be computed assuming different types of boundary conditions, including "natural", "not-a-knot", and "clamped". Details of the derivation of the G matrix is provided in Appendix B4. $h_{x}$ is the increment size in the $x$ direction (for simplicity, here we assume equi-spaced grid points).

The interpolating bicubic spline function $S_{b}(x, y)$ of a two-dimensional function $f(x, y)$ in a box of the grid, defined as the domain $x_{i} \leq x \leq x_{i+1} \cap y_{j} \leq y \leq y_{j+1}$ (the highlighted 
box in Figure A4- 1) is obtained by applying the above method in both dimensions. We first apply the cubic spline in the $x$ direction:

$$
S_{b}(x, y)=P_{1}(x) f\left(x_{i}, y\right)+P_{2}(x) f\left(x_{i+1}, y\right)+P_{3}(x) f_{x x}\left(x_{i}, y\right)+P_{4}(x) f_{x x}\left(x_{i+1}, y\right) \quad \text { Equation A4- } 5
$$

where $f_{x x}\left(x_{i}, y\right)=\sum_{k=1}^{n_{x}} G_{i, k}^{x} f\left(x_{k}, y\right) . f_{x x}$ is the partial second derivative of function $f$ in the $x$ direction. Now, for $y_{j} \leq y \leq y_{j+1}$, we apply the cubic spline in the $y$ direction:

$$
\begin{aligned}
& S_{b}(x, y)=P_{1}(x) {\left[P_{1}(y) f\left(x_{i}, y_{j}\right)+P_{2}(y) f\left(x_{i}, y_{j+1}\right)+P_{3}(y) f_{y y}\left(x_{i}, y_{j}\right)\right.} \\
&\left.+P_{4}(y) f_{y y}\left(x_{i}, y_{j+1}\right)\right]+ \\
& P_{2}(x)\left[P_{1}(y) f\left(x_{i+1}, y_{j}\right)+P_{2}(y) f\left(x_{i+1}, y_{j+1}\right)+P_{3}(y) f_{y y}\left(x_{i+1}, y_{j}\right)\right.\left.+P_{4}(y) f_{y y}\left(x_{i+1}, y_{j+1}\right)\right]+ \\
& P_{3}(x)\left[P_{1}(y) f_{x x}\left(x_{i}, y_{j}\right)+P_{2}(y) f_{x x}\left(x_{i}, y_{j+1}\right)+P_{3}(y) f_{x x y y}\left(x_{i}, y_{j}\right)\right. \\
&\left.+P_{4}(y) f_{x x y y}\left(x_{i}, y_{j+1}\right)\right] \\
&+P_{4}(x)\left[P_{1}(y) f_{x x}\left(x_{i+1}, y_{j}\right)+P_{2}(y) f_{x x}\left(x_{i+1}, y_{j+1}\right)\right. \\
&+\left.P_{3}(y) f_{x x y y}\left(x_{i+1}, y_{j}\right)+P_{4}(y) f_{x x y y}\left(x_{i+1}, y_{j+1}\right)\right]
\end{aligned}
$$

where

$$
f_{y y}\left(x, y_{j}\right)=\sum_{l=1}^{n_{y}} G_{j, l}^{y} f\left(x, y_{l}\right) ; f_{x x y y}\left(x_{i}, y_{j}\right)=\sum_{k=1}^{n_{x}} \sum_{l=1}^{n_{y}} G_{i, k}^{x} G_{j, l}^{y} f\left(x_{k}, y_{l}\right)
$$

Definitions of $G^{y}, P(y)$, and $h_{y}$ are the same as those described for $x$, but in the $y$ direction. Hence, $S_{b}(x, y)$ can be written as:

$$
S_{b}(x, y)=\sum_{\alpha, \beta=1}^{4} A_{\alpha \beta b} P_{\alpha, b}(x) P_{\beta, b}(y)
$$

where $A_{\alpha \beta b}$ are the elements of the following matrix: 


$$
\begin{aligned}
& \boldsymbol{A}_{b} \\
& =\left[\begin{array}{cclc}
f\left(x_{i}, y_{j}\right) & f\left(x_{i}, y_{j+1}\right) & \sum_{l=1}^{n_{y}} G_{j, l}^{y} f\left(x_{i}, y_{l}\right) & \sum_{l=1}^{n_{y}} G_{j+1, l}^{y} f\left(x_{i}, y_{l}\right) \\
f\left(x_{i+1}, y_{j}\right) & f\left(x_{i+1}, y_{j+1}\right) & \sum_{l=1}^{n_{y}} G_{j, l}^{y} f\left(x_{i+1}, y_{l}\right) & \sum_{l=1}^{n_{y}} G_{j+1, l}^{y} f\left(x_{i+1}, y_{l}\right) \\
\sum_{k=1}^{n_{x}} G_{i, k}^{x} f\left(x_{k}, y_{j}\right) & \sum_{k=1}^{n_{x}} G_{i, k}^{x} f\left(x_{k}, y_{j+1}\right) & \sum_{k=1}^{n_{x}} \sum_{l=1}^{n_{y}} G_{i, k}^{x} G_{j, l}^{y} f\left(x_{k}, y_{l}\right) & \sum_{k=1}^{n_{x}} \sum_{l=1}^{n_{y}} G_{i, k}^{x} G_{j+1, l}^{y} f\left(x_{k}, y_{l}\right) \\
\sum_{k=1}^{n_{x}} G_{i+1, k}^{x} f\left(x_{k}, y_{j}\right) & \sum_{k=1}^{n_{x}} G_{i+1, k}^{x} f\left(x_{k}, y_{j+1}\right) & \sum_{k=1}^{n_{x}} \sum_{l=1}^{n_{y}} G_{i+1, k}^{x} G_{j, l}^{y} f\left(x_{k}, y_{l}\right) & \sum_{k=1}^{n_{x}} \sum_{l=1}^{n_{y}} G_{i+1, k}^{x} G_{j+1, l}^{y} f\left(x_{k}, y_{l}\right)
\end{array}\right]
\end{aligned}
$$

Equation

A4- 9

Noting that each element of $A_{b}$ is a linear combination of the values of the function in all the $n_{p}$ points in the grid, $\left\{f_{p}\right\}_{p=1, \ldots, n_{p}}$, Equation A4- 9 takes the form:

$$
S_{b}(x, y)=\sum_{\alpha, \beta=1}^{4} \sum_{p=1}^{n_{p}} E_{\alpha \beta b p} f_{p} P_{\alpha, b}(x) P_{\beta, b}(y)
$$

where $E_{\alpha \beta b p}$ is the coefficient standing by the base $\left\{P_{\alpha, b}(x) P_{\beta, b}(y)\right\}$ in the box $b$, assuming that only a unit current source is placed at the point $p$ in the rectangular grid and the rest of the grid points take the value of zero. The interpolation of the function $f(x, y)$ over the entire domain is therefore achieved from $S_{b}(x, y)$ for different boxes in the domain:

$$
f(x, y)=\left\{\begin{array}{cc}
S_{1}(x, y) & (x, y) \in b_{1} \\
\vdots & \\
S_{n_{b}}(x, y) & (x, y) \in b_{n_{b}}
\end{array}\right.
$$

where $n_{b}=\left(n_{x}-1\right)\left(n_{y}-1\right)$ is the total number of boxes in the grid. Now, using this introduction, we derive the discretized form of Equation A4- 11 in the main text, rewritten below:

$$
V_{e}(t)=\frac{1}{4 \pi \sigma} \iint_{0}^{L} K^{e}(v t+\tau, z) C(\tau, z) d \tau d z
$$


To do so, we discretize the $(\tau, z)$ space into a set of $n_{\tau}$ and $n_{z}$ grid points in the $\tau$ and $z$ directions, respectively. Here, we use "clamped" boundary condition for bicubic splines in both dimensions (i.e. zero derivatives for the boundaries of the domain), since this choice of boundary condition provided the best fit among the other choices including "natural" and "not-a-knot" (data not shown). The final discretized equation can be represented as:

$$
\mathbf{v}=\mathbf{Q} \mathbf{c}
$$

where

$$
\begin{aligned}
& \mathbf{v}=\left[\begin{array}{lll}
\mathbf{v}^{T}\left(\mathrm{t}_{0}\right) & \ldots & \mathbf{v}^{T}\left(\mathrm{t}_{n}\right)
\end{array}\right]^{T} \\
& \mathbf{v}\left(\mathrm{t}_{i}\right)=\left[\begin{array}{lll}
V_{1}\left(\mathrm{t}_{i}\right) & \ldots & V_{n_{e}}\left(\mathrm{t}_{i}\right)
\end{array}\right]^{T} \\
& \mathbf{c}=\left[\begin{array}{lll}
C_{1} & \ldots & C_{n_{p}}
\end{array}\right]^{T}
\end{aligned}
$$

Equation A4- 16

$n_{p}=\mathrm{n}_{\tau} \mathrm{n}_{z}$ is the total number of points in the grid; $\mathbf{v}$ and $\mathbf{c}$ are as described in the main text. The $\mathbf{Q}$ matrix is as defined as follows:

$$
\begin{aligned}
& \mathbf{Q}=\left[\begin{array}{c}
\mathbf{Q}\left(\mathrm{t}_{1}\right) \\
\vdots \\
\mathbf{Q}\left(\mathrm{t}_{n_{t}}\right)
\end{array}\right], \mathbf{Q}\left(\mathrm{t}_{i}\right)=\left[\begin{array}{ccc}
\mathrm{Q}_{11}\left(\mathrm{t}_{i}\right) & \ldots & \mathrm{Q}_{1 n_{p}}\left(\mathrm{t}_{i}\right) \\
\vdots & \ddots & \vdots \\
\mathrm{Q}_{n_{e} 1}\left(\mathrm{t}_{i}\right) & \ldots & \mathrm{Q}_{n_{e} n_{p}}\left(\mathrm{t}_{i}\right)
\end{array}\right] \\
& \mathrm{Q}_{e p}\left(\mathrm{t}_{i}\right)=\frac{1}{4 \pi \sigma} \sum_{b} \iint_{b} K^{e}\left(v \mathrm{t}_{i}+\tau, z\right) B_{p, b}(\tau, z) d \tau d z, \\
& B_{p, b}(\tau, z)=\sum_{\alpha, \beta} E_{\alpha \beta b p} P_{\alpha, b}(\tau) P_{\beta, b}(z)
\end{aligned}
$$

Equation A4- 19

where the integration is over box $b$ (total $\left(n_{\tau}-1\right)\left(n_{z}-1\right)$ boxes in the grid), $n_{t}$ is the total number of time points in the simulation, and $n_{e}$ is the number of electrodes in the probe. The rest of the parameters and functions are as described earlier, but for $\tau$ and $z$. 


\section{APPENDIX B4. DERIVATION OF THE G MATRIX FOR DIFFERENT BOUNDARY CONDITIONS}

The formula stated in Equation A4- 2 guarantees $S(x) \in C^{2}$ in $\left[x_{1}, x_{n}\right]$. The only downside to this formula is that one does not have the knowledge of the second derivative values of the function at grid points. However, since $S(x) \in C^{2}$ in $\left[x_{1}, x_{n}\right]$, the following system provides $(n-2)$ equations relating the second order derivatives at the interior grid points to the function values at the nodes.

$$
S_{i-1}^{\prime}\left(x_{i}\right)=S_{i}^{\prime}\left(x_{i}\right) \quad i=2, \ldots, n-1 \quad \text { Equation B4- } 1
$$

which yields:

$$
\begin{aligned}
h_{x} f^{\prime \prime}\left(x_{i-1}\right)+4 h_{x} f^{\prime \prime}\left(x_{i}\right)+h_{x} f^{\prime \prime}\left(x_{i+1}\right) & i=2, \ldots, n-1 \quad \text { Equation B4- } 2 \\
= & \frac{6}{h_{x}} f\left(x_{i-1}\right)-\frac{12}{h_{x}} f\left(x_{i}\right)+\frac{6}{h_{x}} f\left(x_{i+1}\right)
\end{aligned}
$$

To complete the system of $n$ equations and $n$ unknowns, two additional equations for the second derivatives on the first and last grid points are required. Derivation of these two equations depend on the choice the boundary condition for the domain. Popular choices of boundary conditions are "natural", "clamped", and "not-a-knot". Here we provide the $G$ matrix for each of these types of splines. For simplicity, here we have assumed uniform distribution of the grid points in the domain, however, the methodology can easily be expanded for non-uniform distributions. 


\section{Natural Spline}

In "natural" spline, the second derivative of the function at first and last nodes are assumed to be zero. Hence, the following system of $n$ equations and $n$ unknowns can find second derivative values of the function based on the values of the function at the nodes.

$$
\left\{\begin{array}{l}
f^{\prime \prime}\left(x_{1}\right)=0 \\
\quad h_{x} f^{\prime \prime}\left(x_{i-1}\right)+4 h_{x} f^{\prime \prime}\left(x_{i}\right)+h_{x} f^{\prime \prime}\left(x_{i+1}\right)=\frac{6}{h_{x}} f\left(x_{i-1}\right)-\frac{12}{h_{x}} f\left(x_{i}\right)+\frac{6}{h_{x}} f\left(x_{i+1}\right) \quad \text { Equation B4- 3 } \\
f^{\prime \prime}\left(x_{n}\right)=0
\end{array}\right.
$$

The system of Equation B4- 3 can be written in the matrix format:

$$
\boldsymbol{T}_{\text {nat }} \boldsymbol{f}^{\prime \prime}=\boldsymbol{D}_{\text {nat }} \boldsymbol{f}
$$

Equation B4- 4

where $\boldsymbol{f}^{\prime \prime}=\left[f^{\prime \prime}\left(x_{1}\right), f^{\prime \prime}\left(x_{2}\right), \ldots, f^{\prime \prime}\left(x_{\mathrm{n}}\right)\right]^{T} ; \boldsymbol{f}=\left[f\left(x_{1}\right), f\left(x_{2}\right), \ldots, f\left(x_{n}\right)\right]^{T}$; and $\boldsymbol{T}$ and $\boldsymbol{D}$ are sparse square matrices of length $n$ as follows:

$$
\boldsymbol{T}_{\text {nat }}=\left[\begin{array}{cccccccc}
1 & & & & & & \\
0 & 4 h_{x} & h_{x} & & & & \\
& h_{x} & 4 h_{x} & h_{x} & & & \\
& & h_{x} & 4 h_{x} & h_{x} & & \\
& & & \ddots & \ddots & \ddots & \\
& & & & h_{x} & 4 h_{x} & 0 \\
& & & & & 0 & 1
\end{array}\right]
$$

and

$$
\boldsymbol{D}_{\text {nat }}=\frac{1}{h_{x}}\left[\begin{array}{ccccccc}
0 & 0 & & & & & \\
6 & -12 & 6 & & & & \\
& 6 & -12 & 6 & & & \\
& & 6 & -12 & 6 & & \\
& & & \ddots & \ddots & \ddots & \\
& & & & 6 & -12 & 6 \\
0 & & & 0 & 0
\end{array}\right]
$$

The $\boldsymbol{G}$ matrix for "natural" spline $\left(\boldsymbol{G}_{\text {nat }}\right)$ can then be simply computed from $\boldsymbol{T}_{\text {nat }}$ and $\boldsymbol{D}_{\text {nat }}$ matrices: 


$$
\boldsymbol{G}_{\text {nat }}=\boldsymbol{T}_{\text {nat }}{ }^{-1} \boldsymbol{D}_{\text {nat }}
$$

\section{Clamped Spline}

In "clamped" spline, the derivative of the function at the ends of the domain is assumed to be clamped to specified values (i.e. $f^{\prime}\left(x_{1}\right)=a$; $f^{\prime}\left(x_{1}\right)=b$ ). Without loss of generality, here we assume $a=b=0$. The system of equations can thus be written as follows:

$$
\left\{\begin{array}{l}
2 h_{x} f^{\prime \prime}\left(x_{1}\right)+h_{x} f^{\prime \prime}\left(x_{2}\right)=\frac{-6}{h_{x}} f\left(x_{1}\right)+\frac{6}{h_{x}} f\left(x_{2}\right) \\
h_{x} f^{\prime \prime}\left(x_{i-1}\right)+4 h_{x} f^{\prime \prime}\left(x_{i}\right)+h_{x} f^{\prime \prime}\left(x_{i+1}\right)=\frac{6}{h_{x}} f\left(x_{i-1}\right)-\frac{12}{h_{x}} f\left(x_{i}\right)+\frac{6}{h_{x}} f\left(x_{i+1}\right) \\
h_{x} f^{\prime \prime}\left(x_{n-1}\right)+2 h_{x} f^{\prime \prime}\left(x_{n}\right)=\frac{6}{h_{x}} f\left(x_{n}\right)+\frac{6}{h_{x}} f\left(x_{2}\right)
\end{array}\right.
$$

Hence, $\boldsymbol{T}_{\text {clamp }}$ and $\boldsymbol{D}_{\text {clamp }}$ matrices can be derived as follows:

$$
\boldsymbol{T}_{\text {clamp }}=h_{x}\left[\begin{array}{ccccccc}
2 & 1 & & & & & \\
1 & 4 & 1 & & & & \\
& 1 & 4 & 1 & & & \\
& & 1 & 4 & 1 & & \\
& & & \ddots & \ddots & \ddots & \\
& & & & 1 & 4 & 1 \\
& & & & 1 & 2
\end{array}\right]
$$

and

$$
\boldsymbol{D}_{\text {clamp }}=\frac{1}{h_{x}}\left[\begin{array}{ccccccc}
-6 & 6 & & & & & \\
6 & -12 & 6 & & & & \\
& 6 & -12 & 6 & & & \\
& & 6 & -12 & 6 & & \\
& & & \ddots & \ddots & \ddots & \\
& & & & 6 & -12 & 6 \\
& & & 6 & -6
\end{array}\right]
$$

and similarly,

$$
\boldsymbol{G}_{\text {clamp }}=\boldsymbol{T}_{\text {clamp }}{ }^{-1} \boldsymbol{D}_{\text {clamp }}
$$




\section{Not-a-knot Spline}

In "not-a-knot" spline, the third derivate of the function is assumed to continuous at $x_{2}$ and $x_{n-1}$. The system of equations can thus be written as follows:

$$
\left\{\begin{array}{l}
h_{x} f^{\prime \prime}\left(x_{1}\right)-2 h_{x} f^{\prime \prime}\left(x_{2}\right)+h_{x} f^{\prime \prime}\left(x_{3}\right)=0 \\
h_{x} f^{\prime \prime}\left(x_{i-1}\right)+4 h_{x} f^{\prime \prime}\left(x_{i}\right)+h_{x} f^{\prime \prime}\left(x_{i+1}\right)=\frac{6}{h_{x}} f\left(x_{i-1}\right)-\frac{12}{h_{x}} f\left(x_{i}\right)+\frac{6}{h_{x}} f\left(x_{i+1}\right) \quad \text { Equation B4- 12 } \\
h_{x} f^{\prime \prime}\left(x_{n-2}\right)-2 h_{x} f^{\prime \prime}\left(x_{n-1}\right)+h_{x} f^{\prime \prime}\left(x_{n}\right)=0
\end{array}\right.
$$

Hence, $\boldsymbol{T}_{n k n o t}$ and $\boldsymbol{D}_{n k n o t}$ matrices can be derived as follows:

$$
\boldsymbol{T}_{n k n o t}=h_{x}\left[\begin{array}{ccccccc}
1 & -2 & 1 & & & & \\
1 & 4 & 1 & & & & \\
& 1 & 4 & 1 & & & \\
& & 1 & 4 & 1 & & \\
& & & \ddots & \ddots & \ddots & \\
& & & & 1 & 4 & 1 \\
& & & & 1 & -2 & 1
\end{array}\right]
$$

and

$$
\boldsymbol{D}_{\text {nknot }}=\frac{1}{h_{x}}\left[\begin{array}{ccccccc}
0 & 0 & & & & & \\
6 & -12 & 6 & & & & \\
& 6 & -12 & 6 & & & \\
& & 6 & -12 & 6 & & \\
& & & \ddots & \ddots & \ddots & \\
& & & & 6 & -12 & 6 \\
& & & & & 0 & 0
\end{array}\right]
$$

and similarly,

$$
\boldsymbol{G}_{\text {nknot }}=\boldsymbol{T}_{\text {nknot }}{ }^{-1} \boldsymbol{D}_{\text {nknot }}
$$




\section{APPENDIX C4. COMPARISON OF WAVECSD WITH ICSD AND KCSD METHODS IN EXAMPLE GAUSSIAN GROUND-TRUTH WAVEFORMS}

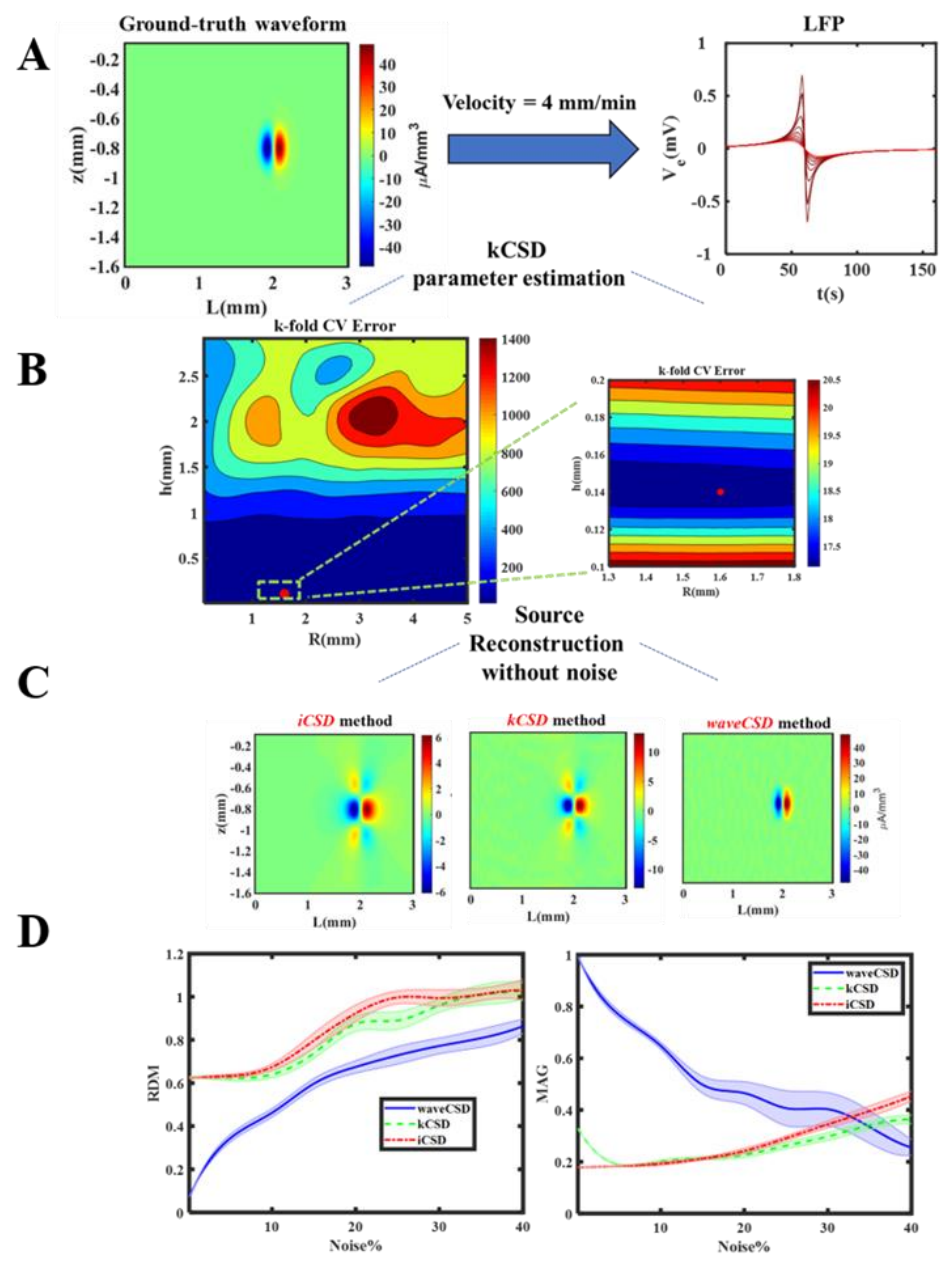

Figure C4- 1 Comparison of the accuracy of waveCSD, iCSD, and kCSD methods in the reconstruction of LFPs generated from a ground-truth waveform. A) A ground-truth waveform (the CSD profile, top left) is assumed to be generated $6 \mathrm{~mm}$ away from an A1x16 probe, inserted perpendicularly in the cortex with an inter-electrode spacing of $100 \mu \mathrm{m}$. The first electrode is assumed to be $100 \mu \mathrm{m}$ deep in the brain tissue. Here, the waveform propagates towards the electrodes at a constant velocity of $4 \mathrm{~mm} / \mathrm{min}$. The resultant LFPs are shown in the top right panel (black $\rightarrow$ most superficial electrode, and red $\rightarrow$ deepest electrode in the probe). B) Model parameter estimation for kCSD method using k-fold cross-validation technique as described in [153]. The left panel shows cross-validation error for a wide range of $\mathrm{R}$ and $\mathrm{h}$ values, and the inset confirms that the selected parameters (the red dot) coincide with the global minimum of cross-validation error values. The estimated diameter (2R) for the kCSD method is used for source reconstruction in the iCSD method shown in panel C. C) Source reconstruction using iCSD, kCSD and waveCSD methods without the presence of noise in the LFP data. (iCSD- RDM: 0.62, MAG: 0.18; kCSD- 0.62, MAG: 0.33, waveCSD- RDM: 0.01, MAG: 0.99). Both RDM and MAG measures confirm that the waveCSD method provides more accurate CSD reconstruction among all methods (i.e. RDM closer to zero and MAG closer to 1 in the waveCSD method). For better visualization, colorbar ranges are different in figures. Parameters: waveCSD methodsame as Figure 4-1. $\mathrm{kCSD}$ method- $\mathrm{R}=1.6[\mathrm{~mm}] ; \mathrm{h}=0.14$. D) mean \pm std for $\mathrm{RDM}$ and MAG of 50 realization of the noisy LFP data at each noise level for all three methods. Noise reduction in the iCSD method was performed by applying a Gaussian spatial filter on the reconstructed CSD from unfiltered LFP recordings (Gaussian filter sigma: $\sigma_{\mathrm{gf}}=0.1[\mathrm{~mm}]$ ). 

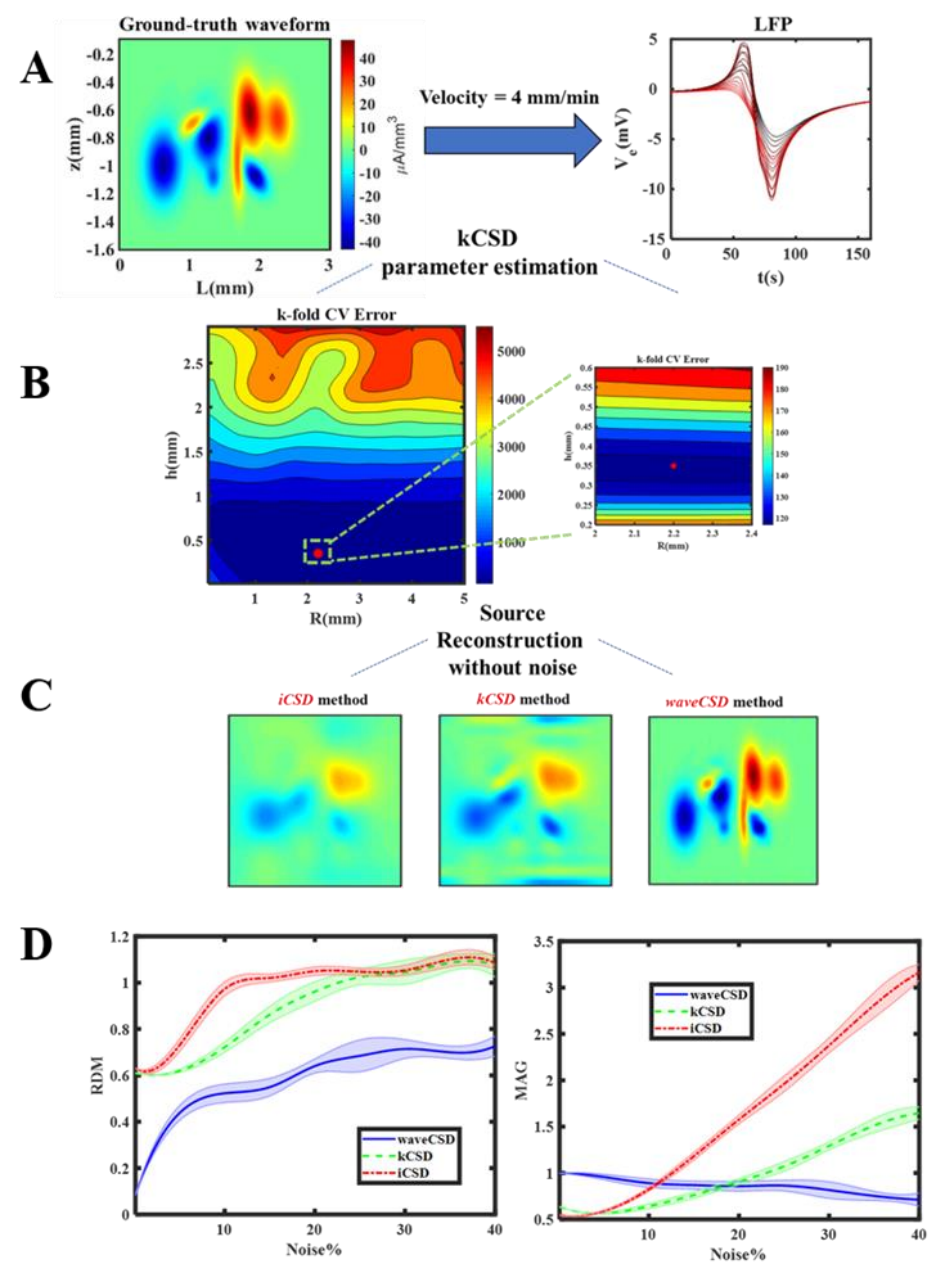

Figure C4- 2 Comparison of the accuracy of waveCSD, iCSD, and kCSD methods in the reconstruction of LFPs generated from a ground-truth waveform. A) A ground-truth waveform (the CSD profile, top left) is assumed to be generated $6 \mathrm{~mm}$ away from an A1x16 probe, inserted perpendicularly in the cortex with an inter-electrode spacing of $100 \mu \mathrm{m}$. The first electrode is assumed to be $100 \mu \mathrm{m}$ deep in the brain tissue. Here, the waveform propagates towards the electrodes at a constant velocity of $4 \mathrm{~mm} / \mathrm{min}$. The waveform is generated by superposition of 10 random Gaussian sources with random amplitude, spread, and angles. The resultant LFPs are shown in the top right panel (black $\rightarrow$ most superficial electrode, and red $\rightarrow$ deepest electrode in the probe). B) Model parameter estimation for kCSD method using k-fold cross-validation technique as described in [153]. The left panel shows cross-validation error for a wide range of $R$ and $h$ values, and the inset confirms that the selected parameters (the red dot) coincide with the global minimum of cross-validation error values. The estimated diameter (2R) for the kCSD method is used for source reconstruction in the iCSD method shown in panel C. C) Source reconstruction using iCSD, kCSD and wave CSD methods without the presence of noise in the LFP data. For comparison purposes, colorbar ranges and labels are the same as in C1 A left panel. (iCSD- RDM: 0.64, MAG: 0.54; kCSD- 0.66, MAG: 0.72, waveCSD- RDM: 0.05, MAG: 0.99). Both RDM and MAG measures confirm that the waveCSD method provides more accurate CSD reconstruction among all methods (i.e. RDM closer to zero and MAG closer to 1 in the waveCSD method). Parameters: waveCSD method- same as Figure 4-1. kCSD method- $\mathrm{R}=2.2$ $[\mathrm{mm}] ; \mathrm{h}=0.35$. D) mean \pm std for RDM and MAG of 50 realization of the noisy LFP data at each noise level for all three methods. Noise reduction in the iCSD method was performed by applying a Gaussian spatial filter on the reconstructed CSD from unfiltered LFP recordings (Gaussian filter sigma: $\sigma_{\mathrm{gf}}=0.1[\mathrm{~mm}]$ ). 


\section{CHAPTER 5 A COMPUTATIONAL ANALYSIS OF THE VASCULAR RESPONSE IN CORTICAL SPREADING DEPRESSION}

\subsection{INTRODUCTION}

As discussed in previous chapters, cortical spreading depression (SD) is a slow wave of neuronal and glial depolarization which results in the cessation of neuronal activity over an extended period of time $[54,55]$. Since its original discovery over 70 years ago by Leao [57] in the rabbit cortex, several research studies have revealed the association of this phenomenon to major neurological disorders including migraine aura, ischemic stroke, traumatic brain injury and SAH $[56,58]$. SD has been shown to greatly disturb ionic and water balances along its path, and is characterized by global release of vasoactive substances and neurotransmitters [54]. Potassium concentrations in the extracellular space, for instance, reach to supra-physiological values $(\sim 30$ to $60 \mathrm{mM}$ from $\sim 3 \mathrm{mM}$ at resting levels), which as described in previous chapters can significantly impact the vascular tone and CBF [55]. During different phases of this wave phenomenon, the levels of ATP, glucose, lactate, and $\mathrm{pH}$ in the brain tissue is also significantly affected. Several studies have demonstrated a mismatch in the metabolic demands and the delivery of oxygen and nutrients to the tissue with huge implications on the disruption of normal NVC and blood perfusion $[9,54,55,59,60]$.

SD involves a variety of mechanisms and cell types. These include neurons, astrocytes, extracellular space, cells in the vascular wall, diffusion and uptake of released neurotransmitters and vasoactive substances [55]. Despite its significance, our knowledge 
of the underlying mechanisms of this phenomenon is still far from ideal and an inclusive understanding of the pathways involved is yet to be achieved. The complexity of SD, as well as the potential feedback and feedforward mechanisms between the involved cell types and perceived pathways, makes studying this subject and the design of proper experiments even more challenging. In parallel to experimental studies, mathematical modeling can be of great assistance in demystifying different aspects of this phenomenon by providing a platform for investigating different cellular pathways and mechanisms without the confounding effect of others. Hence, different aspects of SD have attracted the attention the modeling community over the past few decades.

Using discrete and continuum models, several research groups have focused on neuronal and glial interactions during SD. For instance, Tuckwell and Miura [219], provided a simplified continuum model of SD accounting for the dynamics of potassium and calcium in the intra and extracellular space of neurons to study the importance of extracellular signaling pathways in the propagation of the spreading depression. Shapiro [220], extended the Tuckwell and Miura model by incorporating gap junctions between neurons and astrocytes, and accounted for cell swelling and osmotic forces to identify propagation mechanisms during SD. In a model of gap connected cells, Bennett and colleagues [221] investigated the role of NMDA and glutamate release during in neuronal and astrocytic communications and their role in the propagation of spreading waves in the cortex. There are also studies on the mechanisms of the initiation of SD on detailed models of a single neuron embedded in the extracellular space [222, 223]. More detailed compartmental models have also been developed in recent years, which account for detailed dynamics of 
neurons and astrocytes as well as gap junctions in the propagation of spreading depression wave in the cortex $[217,224]$. A comprehensive review of the mathematical models of SD is available in refs. [225, 226].

Although neurons and glial cells have understandably garnered a great deal of attention in the computational modeling studies of SD, not until very recently by the work of Chang et al. [227] did researchers start to investigate the role of the vascular response in the dynamics of this phenomenon. Authors demonstrated an important role and contribution of vasodilation and vasoconstriction in the regulation of the SD propagation dynamics and its recovery. The work demonstrated how the combined interactions between neurons, astrocytes, and vasculature can result in the disruption of NVC during SD. This study, however, did not address the underlying mechanisms of observed multiphasic vasodilation and constriction in literature $[53,55]$, and used diameter of the vessel as a predefined function of $\left[\mathrm{K}^{+}\right]_{\mathrm{o}}$.

Given the importance of cerebral and intracerebral vessel dynamics in the regulation of $\mathrm{CBF}$, and the presence of ample experimental evidence indicating distinct vascular patterns and large variability within animal species during SD [53, 55], understanding the underlying cellular mechanisms of these responses can undoubtedly advance our knowledge of the phenomenon. In this chapter, we seek to investigate how elevations of $\left[\mathrm{K}^{+}\right]_{\mathrm{o}}$ and the propagation of the spreading wave in the cortex can result in vasoconstriction and vasodilation in different phases of the wave propagation. We use transmembrane current reconstructions estimated in CHAPTER 4 as an indicator of the spatial extent of the SD wavefront and will account for the diffusion of the $\mathrm{K}^{+}$ions in the extracellular 
space, considering the effect of $\mathrm{K}^{+}$uptake by the astrocytes. We study the response of a segment of electrically connected cells, as well as the reconstructed microvascular network used in CHAPTER 1, to a propagating wave of high $\left[\mathrm{K}^{+}\right]_{\mathrm{o}}$.

\section{2 $\left[\mathrm{K}^{+}\right]_{\mathrm{O}}$ PROFILE DURING CORTICAL SPREADING DEPRESSION}

The released $\mathrm{K}^{+}$into the extracellular space is partially taken up by astrocytes, mainly through the activity of inward rectifying potassium (Kir4.1) channels, which serve as a $\mathrm{K}^{+}$ buffer or siphon to transfer these ions from highly concentrated regions to areas of low concentration $[228,229]$. Another important consideration in the diffusion of $\mathrm{K}^{+}$ions in the extracellular space is that the high cellular density in the brain tissue slows down the diffusion rate of $\mathrm{K}^{+}$compared to its free diffusion in an infinite aqueous solution. This restrictive effect of the microenvironment, which was originally introduced by Nicholson and Philips [230, 231], is termed as the tortuosity factor and is estimated to lessens the diffusion rate of $\mathrm{K}^{+}$in the rat brain microenvironment $\sim 2.5$ folds [226, 230, 231]. Therefore, the effective diffusion coefficient of $\mathrm{K}^{+}$in the extracellular space, $\mathrm{D}_{\text {eff }}$, is $\sim 2.5$ times smaller than the diffusion coefficient of $\mathrm{K}^{+}$in an aqueous medium, $\mathrm{D}=2.1 \times 10^{-9}$ $\mathrm{m}^{2} / \mathrm{s}$ at ambient temperature [232].

Assuming a constant $\mathrm{K}^{+}$diffusivity in the extracellular space, and that the SD wave propagation is only in the $\mathrm{x}$ direction, the spatiotemporal profile of $\left[\mathrm{K}^{+}\right]_{\mathrm{o}}$ in front the $\mathrm{SD}$ wavefront can be captured using the second Fick's law of diffusion, Equation 5-1:

$$
\frac{\partial\left[\mathrm{K}^{+}\right]_{\mathrm{o}}}{\partial \mathrm{t}}=\mathrm{D}_{\text {eff }} \frac{\partial^{2}\left[\mathrm{~K}^{+}\right]_{\mathrm{o}}}{\partial \mathrm{x}^{2}}-\mathrm{m}\left[\mathrm{K}^{+}\right]_{\mathrm{o}}
$$


where $\mathrm{m}=8.0 \times 10^{-3} \mathrm{~s}^{-1}[227,233]$ is the potassium buffering rate in astrocytes. The boundary conditions of Equation 5-1 are assumed to be the potassium concentration at the wavefront at $\mathrm{x}=0,(\sim 60 \mathrm{mM})$, and resting $\left[\mathrm{K}^{+}\right]_{\mathrm{o}}$ concentrations $(\sim 3 \mathrm{mM})$ at large distances in front of the wave $(x \rightarrow \infty)$. It is noteworthy that the typical conduction velocities of electrical signals inside the endothelium is in the order of $\mathrm{mm} / \mathrm{s}$ [97] (refer to CHAPTER 2), almost two orders of magnitude larger than the typical velocity of the SD, i.e. in the order of $\mathrm{mm} / \mathrm{min}$ [55]. Since in this chapter we are interested in analyzing the conduction of electrical signals along the brain vasculature, we can safely assume that at each time instance during the propagation of the spreading wave, the vascular response has reached to its steady state profile, i.e. the quasi-steady state approach. Therefore, instead of solving the partial differential equation of Equation 5-1, we assume that the steady state profile of $\left[\mathrm{K}^{+}\right]_{\mathrm{o}}$ is propagated along the vascular network with the velocity of the spreading depression wave.

Figure 5-1 A shows the steady state solution of Equation 5-1 with boundary conditions stated earlier. The solution is an exponential decay of $\left[\mathrm{K}^{+}\right]_{\mathrm{o}}$ with a length constant of $\sqrt{\frac{D_{e f f}}{m}}$ $\sim 300 \mu \mathrm{m}$. Additionally, based on our analysis in CHAPTER 4, we choose a spatial extent of $\sim 200 \mu \mathrm{m}$ for the wavefront, during which $\left[\mathrm{K}^{+}\right]_{\mathrm{o}}$ is at its maximum concentration $(60 \mathrm{mM})$. We also assume a symmetrical profile in the diffusion of $\mathrm{K}^{+}$to the left and right of the SD wavefront (Figure 5-1 B). This spatial profile of $\left[\mathrm{K}^{+}\right]_{\mathrm{o}}$ will be utilized in subsequent simulations, to predict the response of vessels to a wave of SD. 
A

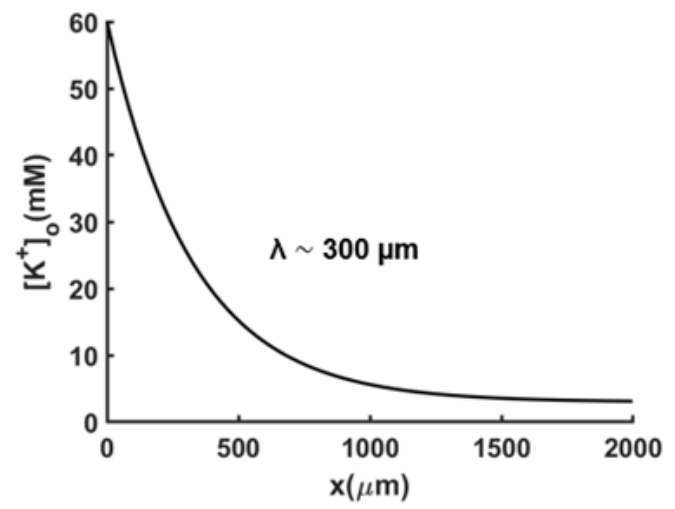

B

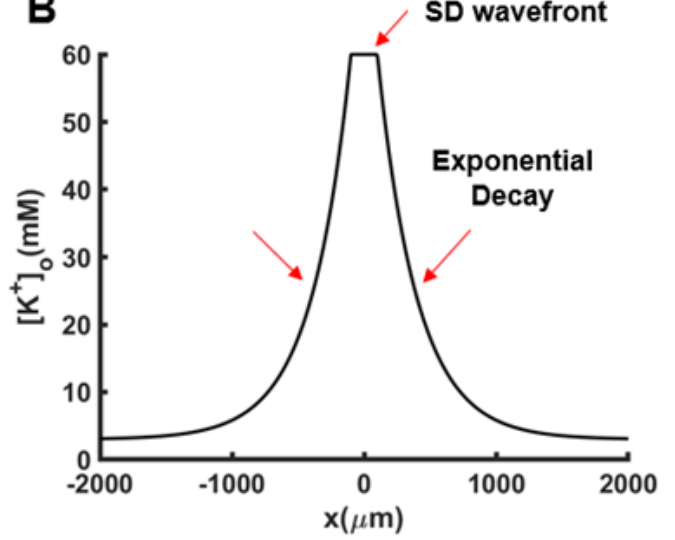

Figure 5-1 Profile of $\mathrm{K}^{+}$ions in the extracellular space during cortical spreading depression. (A) Steady state solution of Equation 5-1, showing the diffusion profile of $\mathrm{K}^{+}$ions in the extracellular space in front of the SD wavefront. $\left[\mathrm{K}^{+}\right]_{\mathrm{o}}$ is assumed to be $60 \mathrm{mM}$ at the wavefront and $3 \mathrm{mM}$ at large distances in front of the wave $(x \rightarrow \infty)$. The diffusion profile follows an exponential decay with the length constant of $300 \mu \mathrm{m}$. (B) An instance of the $\left[\mathrm{K}^{+}\right]_{\text {o }}$ profile along the length of the vessel assuming that the SD wavefront is at the origin in the $\mathrm{x}$ direction. The profile assumes the duration of the wave to be $\sim 200 \mu \mathrm{m}$. $\left[\mathrm{K}^{+}\right]_{\mathrm{o}}$ decays exponentially before and after the wave based on the profile in panel (A).

\subsection{RESPONSE OF BRAIN VASCULATURE TO A WAVE OF HIGH [K+ $\left.{ }^{+}\right]$}

A single layer of representative endothelial cells is constructed by electrically connecting cells through gap junctions. Each cell is modeled using a minimal modelling approach discussed in CHAPTER 2, where the cell contains a Kir current and the rest of membrane currents are lumped into a non-specific linear background current $\left(\mathrm{I}_{\mathrm{bg}}\right)$, with the lumped conductance $\mathrm{G}_{\mathrm{bg}}$. As discussed in detail in CHAPTER 2, the dynamics of the membrane potential of the system can be studied using the following set of equations:

$$
\begin{aligned}
& C_{m, i} \frac{d V_{m, i}}{d t}=-I_{b g, i}-I_{K i r, i}-I_{g j, i} \quad i=1, \ldots, N \\
& I_{g, i}=\sum_{n} \frac{1}{R_{g j, i, n}}\left(V_{m, i}-V_{m, n}\right) \\
& I_{\text {Kir }}=\frac{\bar{G}_{\text {Kir }}\left[K^{+}\right]_{o}^{0.5}\left(V_{m}-E_{K}\right)}{1+\exp \left(\frac{\left(V_{m}-V_{0.5}\right)}{k}\right)}
\end{aligned}
$$

Equation 5-4 


$$
I_{b g}=G_{b g}\left(V_{m}-E_{b g}\right)
$$

Where definitions of the parameters are the same as in CHAPTER 2. Figure 5-2 A demonstrates the steady state response of the cell layer when a static spatial profile of $\left[\mathrm{K}^{+}\right]_{\mathrm{o}}$ in Figure 5-1 B is imposed on cells. Cells shown in blue were exposed to $60 \mathrm{mM}$ of $\left[\mathrm{K}^{+}\right]_{\mathrm{o}}$, corresponding to the SD wavefront (approximately 10 cells or $\sim 200 \mu \mathrm{m}$, assuming a length of $20 \mu \mathrm{m}$ for each cell), and cells in gray were subjected to the exponential decay of $\mathrm{K}^{+}$ ions before and after the wave, see Figure 5-1 B. The temporal evolution of the membrane potential of cells is depicted in Figure 5-2 B. For the simulations performed in this figure, the $\mathrm{G}_{\mathrm{Kir}}$ to $\mathrm{G}_{\mathrm{bg}}$ ratio of cells was chosen in the yellow region (Figure 5-2 B inset), which as discussed in CHAPTER 2, promotes passive conduction of the electrical signal along the length of the vessel.
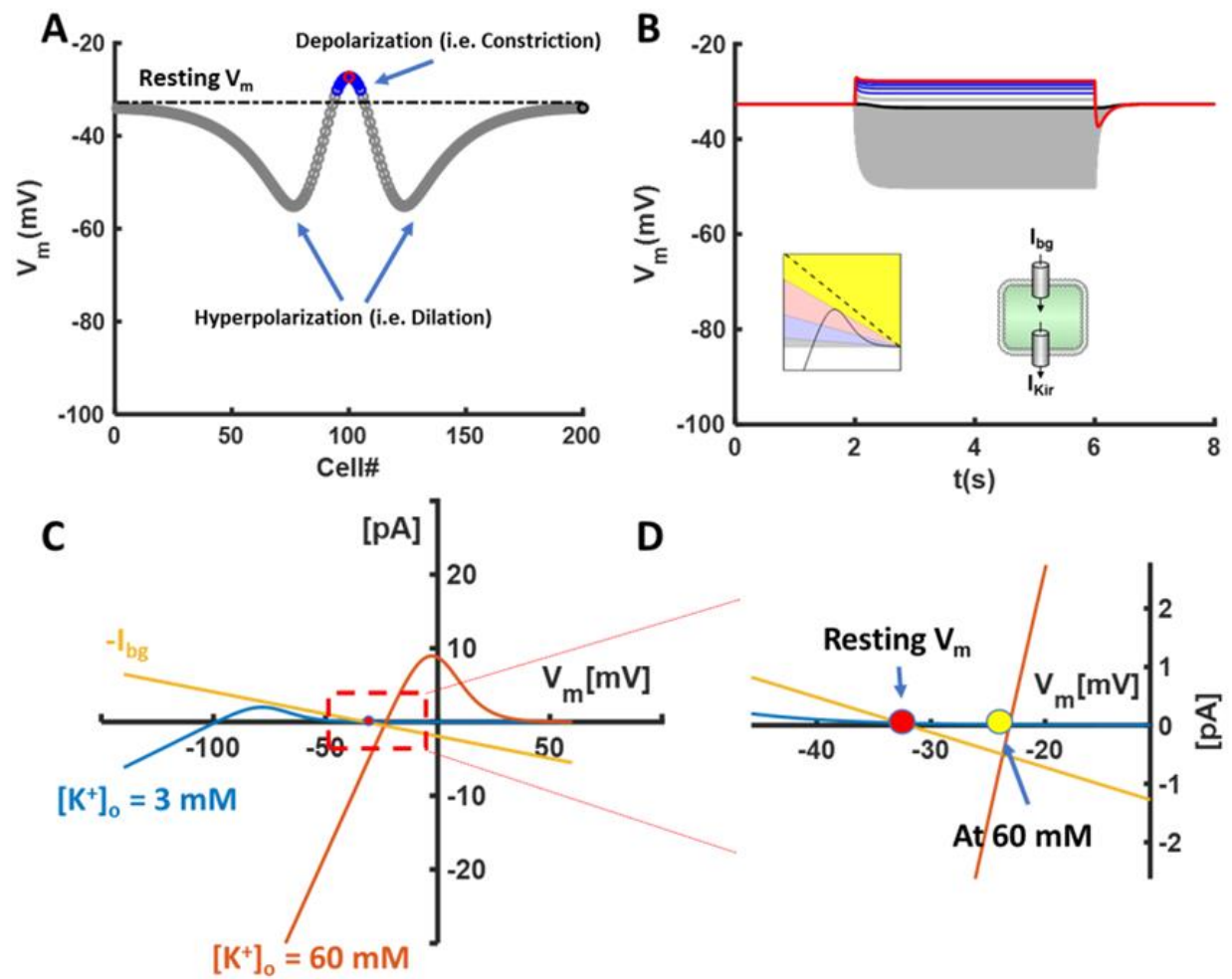

Figure 5-2- Response of a segment of endothelial cells to elevations of $\left[\mathrm{K}^{+}\right]_{0}$ during SD. An array of 200 identical cells are connected via gap junctions and are subjected to the stationary $\left[\mathrm{K}^{+}\right]_{\mathrm{o}}$ profile of Figure 5-1 
B. Each cell is assumed to be $20 \mu \mathrm{m}$ in length. (A) steady state response of the system to elevations in $\left[\mathrm{K}^{+}\right]_{\mathrm{o}}$ during SD. The blue circle denote cells on the wavefront (with the spatial extent of $200 \mu \mathrm{m}$ and $\left[\mathrm{K}^{+}\right]_{\mathrm{o}}=60$ $\mathrm{mM}$ ), and gray cells are subjected to the exponential decay of $\left[\mathrm{K}^{+}\right]_{0}$ before and after the wave (Figure 5-1 B). The black dashed line shows the resting $\mathrm{V}_{\mathrm{m}}$ of cells before the elevation of $\left[\mathrm{K}^{+}\right]_{\mathrm{o}}$. (B)Temporal response of the cells to the $\left[\mathrm{K}^{+}\right]_{\mathrm{o}}$ profile of Figure 5-1 B. The blue, red, and gray traces correspond to the cells highlighted in panel (A). The right figure inset show that each cell is modeled with a Kir and a bg current. The left figure inset shows the relative position of the Kir and bg conductance at rest for the simulations in this figure (the yellow region). (C) I_V curves of Kir and bg currents at rest, and upon application of $60 \mathrm{mM}\left[\mathrm{K}^{+}\right]_{\mathrm{o}}$. The red dot shows the stable steady state of the system at resting conditions $\left(\left[\mathrm{K}^{+}\right]_{0}=3 \mathrm{mM}\right)(\mathrm{D})$ a Magnified region of panel (C), indicating the steady state of the system at rest and upon $\left[\mathrm{K}^{+}\right]_{\mathrm{o}}=60 \mathrm{mM}$. Red dot is the stable steady state at rest, and the yellow dot is the stable steady state upon potassium stimulation. Parameters used for the simulations in this figure: $\overline{\mathrm{G}}_{\mathrm{Kir}}=0.2 \mathrm{nS} / \mathrm{mM} 0.5, \mathrm{G}_{\mathrm{bg}}=0.06 \mathrm{nS}, \mathrm{E}_{\mathrm{bg}}=-30 \mathrm{mV},\left[\mathrm{K}^{+}\right]_{\mathrm{i}}=150 \mathrm{mM}, \mathrm{V}_{0.5}$ $=\mathrm{E}_{\mathrm{K}}+25 \mathrm{mV}, \mathrm{C}_{\mathrm{m}}=8 \mathrm{pF}, \mathrm{R}_{\mathrm{gj}}=50 \mathrm{M} \Omega$.

Interestingly, results show that while high elevations of $\left[\mathrm{K}^{+}\right]_{\mathrm{o}}$ at the wavefront causes depolarization of the vessel, which result in vasoconstriction, regions immediately before and after the wavefront will undergo hyperpolarization, or vasodilation. Under the conditions assumed for these simulations, the changes in the membrane potential of cells exponentially decrease as cells are farther away from the wavefront (notice the decay of the signal after the peak hyperpolarization). The voltage current relationship of $\mathrm{I}_{\mathrm{Kir}}$ and $\mathrm{I}_{\mathrm{bg}}$ is shown in panel $\mathrm{C}$ under both extreme cases of $\left[\mathrm{K}^{+}\right]_{\mathrm{o}}$, i.e. at rest $(3 \mathrm{mM}$; the blue curve) and under high elevations during SD (60 mM; the orange curve). As can be observed in panel D (the magnified version of panel C), under resting conditions, the only steady state of the system is at $\sim-30 \mathrm{mV}$ (the red circle; intersection between $\mathrm{I}_{\mathrm{kir}}$ and $-\mathrm{I}_{\mathrm{bg}}$ ). Upon elevation of $\left[\mathrm{K}^{+}\right]_{\mathrm{o}}$ to $60 \mathrm{mM}$, however, Kir current is shifted way to more depolarized potentials (due to the change in $\mathrm{E}_{\mathrm{K}}$ from $\sim-100 \mathrm{mV}$ at rest to $\sim-22 \mathrm{mV}$ during high potassium), and the new steady state of the system is highlighted as the yellow circle. Since the new steady state is more depolarized than the original steady state, the cell will undergo depolarization in response to potassium stimulation. Also evident is the increase in the Kir current amplitude owing to the dependence of the single channel conductance on $\left[\mathrm{K}^{+}\right]_{\mathrm{o}}$ (Equation 5-4, refer to CHAPTER 2). As the $\left[\mathrm{K}^{+}\right]_{\mathrm{o}}$ for the rest of the cells (gray cells) are 
between the wavefront and resting conditions, the Kir and bg relationship of these cells will therefore fall between two extreme cases shown in Figure 5-2 C. Therefore, depending on the level of potassium stimulus, cells can either depolarize or hyperpolarize.

Similar simulations are performed in Figure 5-3 with the difference that the Kir to bg ratio of these cells are chosen such that cells promote regenerative conduction of electrical signals (the blue region in Figure 5-3 A inset; refer to CHAPTER 2 for the comparison of passive vs. regenerative cells). The $\left[\mathrm{K}^{+}\right]_{\mathrm{o}}$ profile is identical to that in Figure 5-2. While similar to the earlier results, cells at the wavefront (blue circles) show depolarization of membrane potential (equivalent to vasoconstriction), cells before and after the wavefront showed massive levels of hyperpolarization, which persisted even in cells far away from the wavefront (Figure 5-3A). These results suggest that the hyperpolarization of cells adjacent to the SD wavefront initiated a hyperpolarizing wave that regeneratively propagated along the vessel length in both directions. This regenerative conduction of the signal was possible since cells have higher Kir current densities compared to the simulations in Figure 5-2 (the blue region in Figure 5-3 A inset). Kir and bg current curves shown in Figure 5-3 C,D show that cells are bistable and hyperpolarizing favoring with two stable steady states at rest, and one during high $\left[\mathrm{K}^{+}\right]_{\mathrm{o}}$. Results indicate that cells before and after the wavefront pass the $\mathrm{V}_{\mathrm{m}}$ threshold required for the hyperpolarization jump (saddle-node bifurcation) to their hyperpolarized state. 

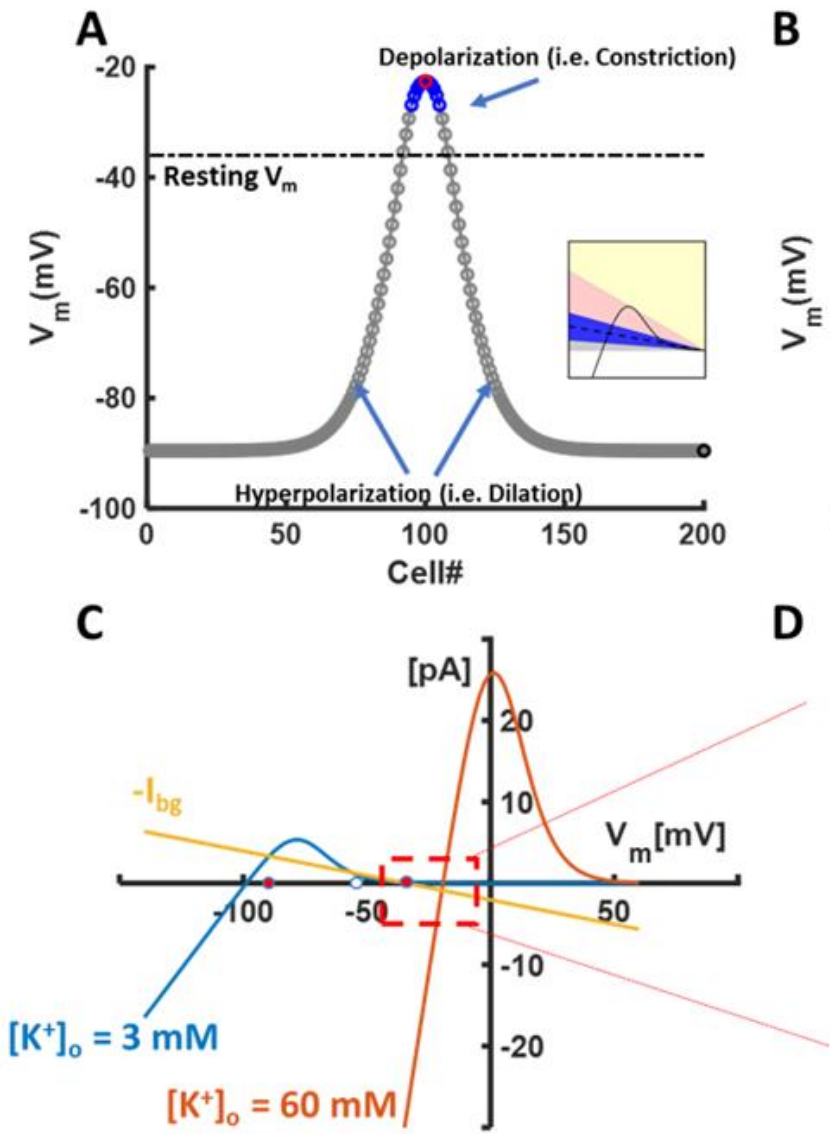

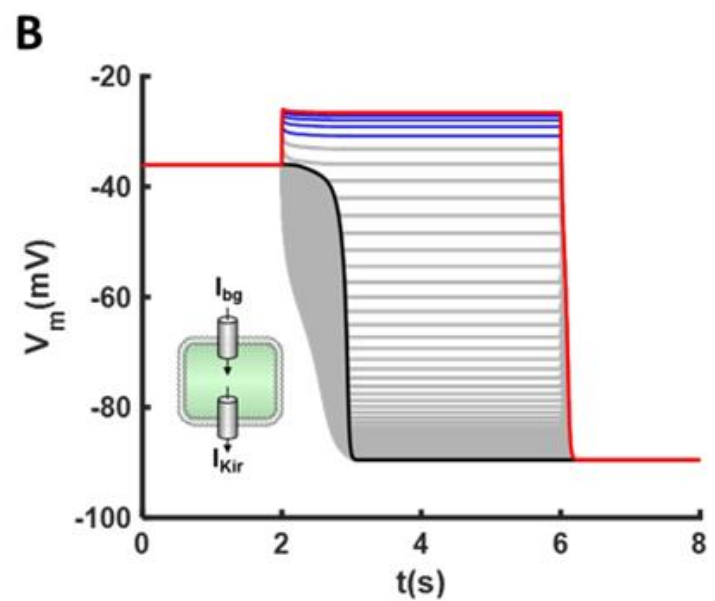

D

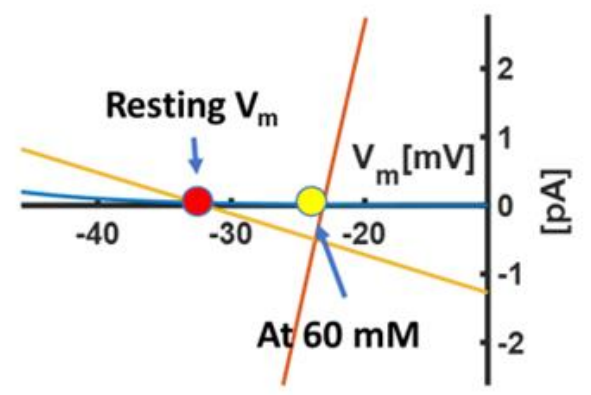

Figure 5-3- Response of a segment of endothelial cells to elevations of $\left[\mathrm{K}^{+}\right]_{0}$ during SD. An array of 200 identical cells are connected via gap junctions and are subjected to the stationary $\left[\mathrm{K}^{+}\right]_{\mathrm{o}}$ profile of Figure 5-1 B. Each cell is assumed to be $20 \mu \mathrm{m}$ in length. (A) Steady state response of the system to elevations in $\left[\mathrm{K}^{+}\right]_{\mathrm{o}}$ during SD. The blue circle denote cells on the wavefront (with the spatial extent of $200 \mu \mathrm{m}$ and $\left[\mathrm{K}^{+}\right]_{\mathrm{o}}=60$ $\mathrm{mM}$ ), and gray cells are subjected to the exponential decay of $\left[\mathrm{K}^{+}\right]_{\mathrm{o}}$ before and after the wave (Figure 5-1 B). The black dashed line shows the resting Vm of cells before the elevation of $\left[\mathrm{K}^{+}\right]_{\mathrm{o}}$. (B)Temporal response of the cells to the $\left[\mathrm{K}^{+}\right]_{\mathrm{o}}$ profile of Figure 5-1 B. The blue, red, and gray traces correspond to the cells highlighted in panel (A). The right figure inset show that each cell is modeled with a Kir and a bg current. The left figure inset shows the relative position of the Kir and bg conductance at rest for the simulations in this figure (the blue region). (C) I_V curves of Kir and bg currents at rest, and upon application of $60 \mathrm{mM}\left[\mathrm{K}^{+}\right]_{\mathrm{o}}$. The red dots show the stable steady states of the system at resting conditions $\left(\left[\mathrm{K}^{+}\right]_{\mathrm{o}}=3 \mathrm{mM}\right)$, and the white dot indicate the unstable steady state. (D) a Magnified region of panel (C), indicating the steady state of the system at rest and upon $\left[\mathrm{K}^{+}\right]_{\mathrm{o}}=60 \mathrm{mM}$. Red dot is the stable steady state at rest, and the yellow dot is the stable steady state upon potassium stimulation. Parameters used for the simulations in this figure: $\bar{G}_{\text {Kir }}=0.4$ $\mathrm{nS} / \mathrm{mM} 0.5, \mathrm{G}_{\mathrm{bg}}=0.06 \mathrm{nS}, \mathrm{E}_{\mathrm{bg}}=-30 \mathrm{mV},\left[\mathrm{K}^{+}\right]_{\mathrm{i}}=150 \mathrm{mM}, V_{0.5}=\mathrm{EK}+25 \mathrm{mV}, \mathrm{C}_{\mathrm{m}}=8 \mathrm{pF}, \mathrm{R}_{\mathrm{gg}}=50 \mathrm{M} \Omega$.

To simulate the response of the vessel segment to a wave of SD, the steady state profile of

Figure 5-1 B is propagated along the vessel. The vessel response is shown at four representative time instances of the wavefront location in Figure 5-4. In both panels of this figure, the same distribution of cell membrane potentials in Figure 5-2 and Figure 5-3 were 
observed at each location of the wavefront. Interestingly, if one considers a fixed location along the vessel (the red dashed line, for instance), both panels indicate that the vessel undergoes dilation before the arrival of the wave (cells are hyperpolarized), constriction upon the arrival (depolarization of the cells), and again dilation when the wave has passed (cells become hyperpolarized). Model simulations predict that if cells are regenerative and hyperpolarizing favoring (Figure 5-4 B), the hyperpolarization after the passage of the wave will continue for a long time, since cells have undergone regenerative conduction of hyperpolarization. As discussed in great depth in CHAPTER 2, additional mechanisms, e.g., activity of the sodium potassium pump or delayed activation of depolarizing currents, might be needed for the system to recover. This finding might suggest an explanation for the observed long post SD hyper perfusion in experiments $[53,55]$. Conversely, if cells are passive (Figure 5-2 A), the hyperpolarization level reduces as the wave propagates to more distant areas, and cells will eventually return to their resting $\mathrm{V}_{\mathrm{m}}$ (or equivalently, to their resting diameter) after shorter time durations. 


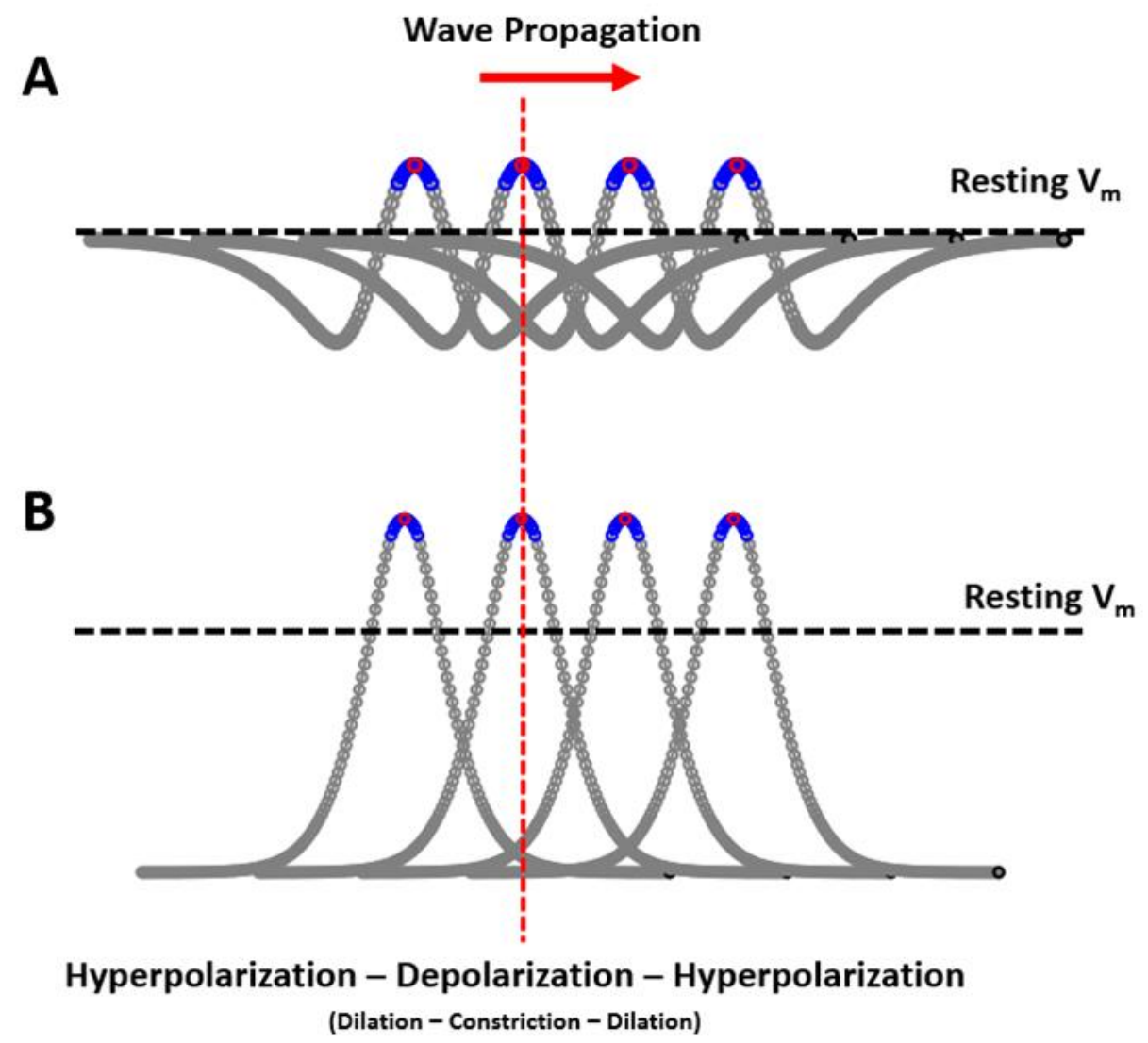

Figure 5-4 Response of a segment of endothelial cells to a propagating wave of high $\left[\mathrm{K}^{+}\right]_{\mathrm{o}}$ during SD.(A) The conditions of the simulations in this panel is identical to those of Figure 5-2 (where cells are in the yellow (passive) regions) with the difference that the $\left[\mathrm{K}^{+}\right]_{\mathrm{o}}$ profile is propagated along the vessel with a velocity of $3 \mathrm{~mm} / \mathrm{min}$ (corresponding to the velocity of the SD). At four representative time instances, the voltage profile of the cells is plotted where the resting $\mathrm{V}_{\mathrm{m}}$ is shown as the black dashed line. The propagation is assumed to be from left to right. (B) same procedure as in panel (A) is repeated for the conditions in Figure 5-3 (where cells are in the blue (regenerative) region). Both panels show that the at each time instance, the profile of the membrane potential of cells is almost identical to the steady state responses shown in Figure 5-2 and Figure 5-3. At a fixed location along the vessel, e.g. the red dashed line, as the wave propagates from left to right, the vessel undergoes hyperpolarization before the wavefront (i.e. dilation), depolarization upon the wave arrival (i.e. constriction), and again hyperpolarization after the passage of the wave (i.e. dilation).

Simulations in a large reconstructed network from the somatosensory cortex of rats (reconstructed from [96]), consisting of interconnected brain capillaries, parenchymal arterioles, and parenchymal venules (Figure 5-5 B), also revealed similar results to those in previous figures when cells were exposed to a wave of high $\left[\mathrm{K}^{+}\right]_{\mathrm{o}}$ (Figure 5-5). Each arteriolar cell is modeled as three ECs coupled to three SMCs at each longitudinal position. Each PA EC-SMC unit has an effective leak conductance, reflecting a net transmembrane 
resistance $\left(\mathrm{G}_{\mathrm{bg}, \mathrm{PA}}=1 / \mathrm{R}_{\mathrm{m}, \mathrm{EC}-\mathrm{SMC}}\right.$; where $\mathrm{R}_{\mathrm{m}, \mathrm{EC}-\mathrm{SMC}}$ is the membrane resistance of the PA ECSMC unit), and a total Kir conductance $\left(\overline{\mathrm{G}}_{\mathrm{Kir}, \mathrm{PA}}=\overline{\mathrm{G}}_{\mathrm{Kir}, \mathrm{EC}}+\overline{\mathrm{G}}_{\mathrm{Kir}, \mathrm{SMC}}\right)$. Each unit is coupled to adjacent EC-SMC units with a resistance, $\mathrm{R}_{\mathrm{gj}, \mathrm{PA}}$. The definitions of the parameters are the same as CHAPTER 2.

In these simulations, we assumed that the propagation of SD is solely in the $\mathrm{x}$ direction, and presumed SD as a planar wave (Figure 5-5 B; the highlighted purple region shows the duration of the wavefront). Hence, the $\left[\mathrm{K}^{+}\right]_{\mathrm{o}}$ profile is constant in the $\mathrm{y}$ direction, and along the $\mathrm{x}$ direction it is identical to the profile shown in Figure 5-1 B. Results show that vessels undergo hyperpolarization or depolarization regardless of their arrangement compared to the direction of SD wave propagation (Figure 5-5 A), which points to the existence of mechanisms involving the conduction of electrical signals along the vessels during SD. Although in these simulations we are bounded by the physical limits of the network under analysis, the resultant membrane potential profiles before, during, and after the wavefront is almost identical to the representative single endothelial layer in Figure 5-2 and Figure 5-3 (notice hyperpolarization before and after the wavefront in the top view of Figure 5-5 $\mathrm{C}$ and in voltage traces in Figure 5-5 D). These results are in keepings with the observation of Brennan et al. [53] in rats for the existence of a triphasic response (dilation, constriction, and dilation), during the propagation of SD. 

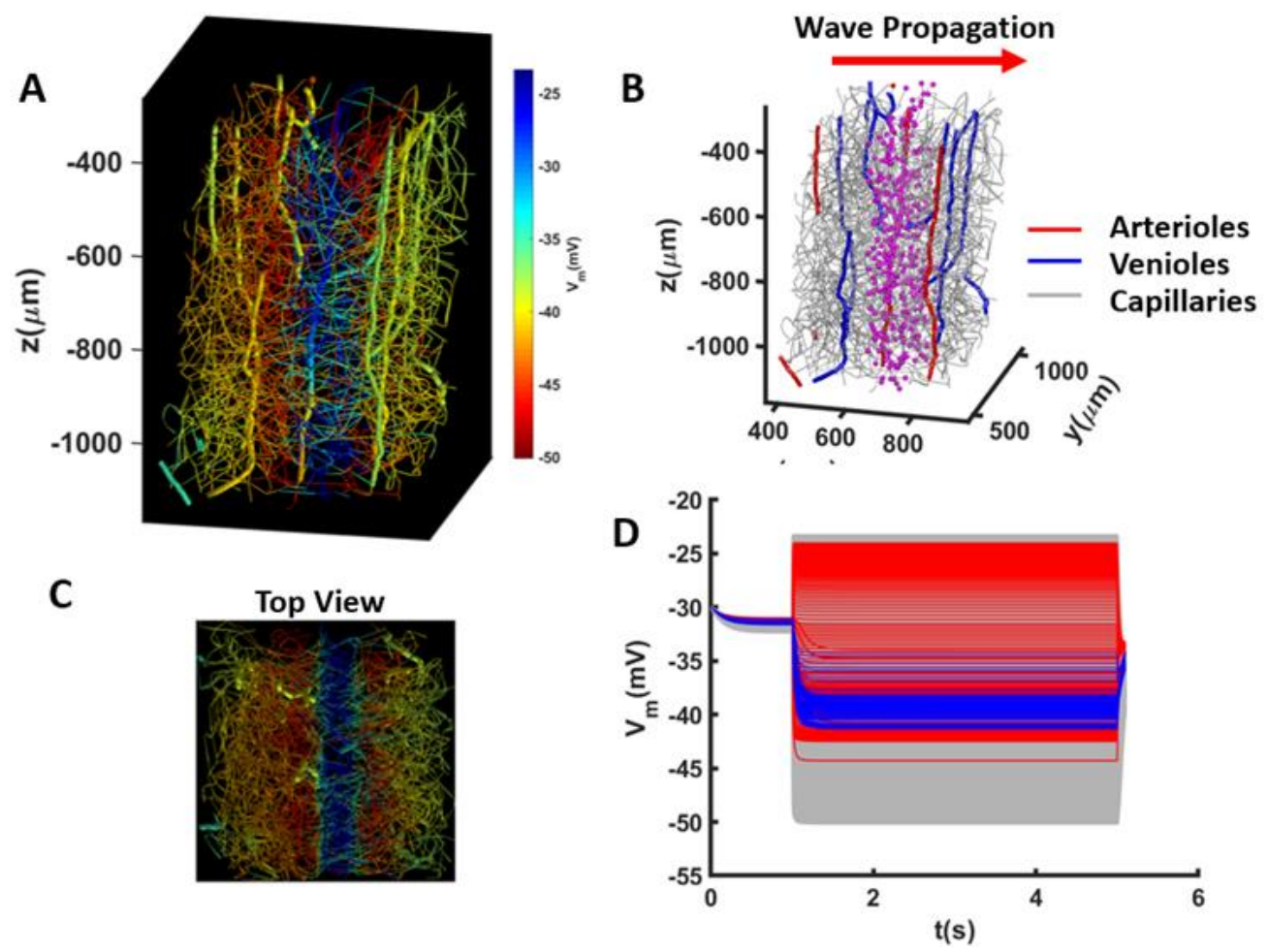

Figure 5-5 Response of a large network of brain vasculature (reconstructed from [96]) to the stationary profile of high $\left[\mathrm{K}^{+}\right]_{\mathrm{o}}$ during SD. (A) The steady state response of the system when the stationary profile of $\left[\mathrm{K}^{+}\right]_{\mathrm{o}}$ in Figure 5-1 B is imposed on the cells. (B) The structure and size of the network. Capillaries are demonstrated as gray, PAs as red, and PVs as blue. The highlighted purple dots show the SD wavefront $(\sim 200 \mu \mathrm{m}$ in dimension). The wave is assumed to propagate only in the $\mathrm{x}$ direction and is assumed to be planar. (C) Top view of the response of the steady state response of the system shown in panel (A). (D) Temporal distribution of the membrane potential of cells (colors match panel (B)), in response to the stationary $\left[\mathrm{K}^{+}\right]_{\mathrm{o}}$ profile. Parameters for the capillaries are as stated in Figure 5-2, for PA: $\overline{\mathrm{G}}_{\mathrm{Kir}, \mathrm{PA}}=0.4 \mathrm{nS} / \mathrm{mM}^{0.5}, \mathrm{G}_{\mathrm{bg}}=0.2 \mathrm{nS}, \mathrm{E}_{\mathrm{bg}}=$ $-30 \mathrm{mV}, \mathrm{V}_{0.5}=\mathrm{E}_{\mathrm{K}}+25 \mathrm{mV}$, and $\mathrm{R}_{\mathrm{g}, \mathrm{PA}}=50 \mathrm{M} \Omega$, for PV: $\overline{\mathrm{G}}_{\mathrm{Kir}, \mathrm{PV}}=0 \mathrm{nS} / \mathrm{mM}^{0.5}, \mathrm{G}_{\mathrm{bg}}=0.2 \mathrm{nS}, \mathrm{E}_{\mathrm{bg}}=-30 \mathrm{mV}$, and $\mathrm{R}_{\mathrm{gj}, \mathrm{PV}}=50 \mathrm{M} \Omega$.

\subsection{DISCUSSION}

Several studies in literature show massive changes in the hemodynamic response of different animal species during and after the propagation of SD [55]. These changes have been classified into a combination of a varying degree of four distinct vasomotor components (depending on the type of species, vessel type, and the SD order). These phases include: I) the initial local hypoperfusion or vasoconstriction coincident with the start of the DC shift in the local field potentials, II) a peak hyperemia following the initial 
vasoconstriction, III) a late smaller hyperemia with lower levels of CBF increase compared to the peak hyperemia, and in some cases IV) a delayed post-SD vasoconstriction and oligemia [234]. In a pioneering study, Brennan et al [53], using simultaneous recordings of the optical reflectance changes during SD along with the electrophysiological recordings of local field potentials in rats, also observed a vasodilation phase prior to the arrival of the wave, which was followed by the local hypoperfusion, and at later stages by vasodilation. The results shown in model simulations performed in this chapter were in agreement with these observations (Figure 5-2 to Figure 5-5) and provided a potential mechanism for the observed multiphasic vascular response during SD.

Although we specifically focused on the role of increases in $\left[\mathrm{K}^{+}\right]_{\mathrm{o}}$ as the major vasoactive component in an overly simplified model of propagating SD, a large number of other vasoactive substances, including AA metabolites, prostaglandins and NO, are also released during SD $[54,55]$. The competing effects of these mediators may provide an explanation for the observed variability in the vascular and hemodynamic responses in literature. For instance, the known vasodilatory effect of NO, or other vasodilatory AA metabolites [17], can create a tug of war with the vasoconstrictive effect of high levels of $\left[\mathrm{K}^{+}\right]_{\mathrm{o}}$ during the initial phases of SD propagation, which can determine the state of the vessel, as well as the degree of the change in $\mathrm{CBF}$. Other factors, for instance cell swelling and resultant shrinkage in the extracellular space $[54,55]$, may result in the activation of mechanosensitive channels, e.g. non selective cation channels in different vascular beds, which can further change the response of the vasculature in SD. Also, since membrane potential of cells undergo large changes during $\mathrm{SD}$, voltage-gated $\mathrm{K}^{+}$and most importantly $\mathrm{Ca}^{2+}$ 
channels may also play a significant role in determining the vessel dynamics. Inclusion of these pathways, however, was beyond the scope of the work presented in this chapter and will be a subject of future investigations.

Results presented here predict the pivotal role of Kir channels in the observed multiphasic response of vasculature during SD-associated high elevations of $\left[\mathrm{K}^{+}\right]_{\mathrm{o}}$ (Figure 5-2 to Figure 5-5). Results explain how massive increases in $\left[\mathrm{K}^{+}\right]_{0}$ change the relative positioning of the Kir curve with respect to the bg current (Figure 5-2 and Figure 5-3, panels C,D). This causes the depolarization of cells at the SD wavefront, as well as cells immediately adjacent to it. Also, model simulations show that since cells in both sides of the wavefront receive sub-maximal levels of $\left[\mathrm{K}^{+}\right]_{\mathrm{o}}$, due to the diffusion of the $\mathrm{K}^{+}$in the extracellular space, Kir channels can initiate and conduct a hyperpolarizing (vasodilatory) signal which is propagated along the vessel (Figure 5-2 and Figure 5-3). The spatial extent of this propagation depended on relative Kir density in the cells (compared to the bg current) (compare Figure 5-2 and Figure 5-3) and the degree of potassium buffering in the medium. These results, along with simulations in Figure 5-5, corroborated the observations of Brennan et al. [53], that the conducted responses in vasculature did not follow the path of the SD wave; rather, they propagated along the path of the vessel. Also, in agreement with experimental observations that the conducted responses never skipped a vessel along it path, our simulations show that hyperpolarization or depolarization of the membrane potential propagated in all vessels of the region affected by the wavefront as well as in the neighboring areas (Figure 5-5). 
Overall, although undoubtedly simplified, model simulations performed in this chapter reiterate the importance of the dynamics of $\mathrm{K}^{+}$ions and channels, in particular inward rectifying potassium, in the regulation of the vascular tone and CBF. Model simulations predict a key role of Kir channels in the multiphasic responses of brain vasculature during various phases of SD. 


\section{CHAPTER 6 HEMODYNAMIC RESPONSE DURING NEUROVASCULAR COUPLING}

The content of this chapter is to be submitted as Moshkforoush A, Ashenagar B, Mirza A, Tsoukias M. "Multiscale Model of Cerebral Blood Flow Control”.

In previous chapters we investigated different mechanisms of NVC under normal and diseased conditions. We analyzed the role of capillaries as sensors of neuronally-induced increases in $\left[\mathrm{K}^{+}\right]_{\mathrm{o}}$ and studied how signals from different regions of the brain can result in upstream propagation of electrical signals that dilate feeding arterioles. Also, we specified how the myogenic tone of PAs can be dysregulated under the presence of pathological conditions or upon changes in the extracellular milieu. The work presented in this chapter aims to understand how changes in the diameter of parenchymal arterioles affect the hemodynamic response, i.e., distribution of blood flow and hematocrit, in different regions of complex brain microvascular networks.

\subsection{INTRODUCTION}

Under physiological conditions, CBF is actively regulated to deliver oxygen-rich red blood cells to different brain regions to meet changing tissue demands. Blood flow is also a means of removing by-products of cerebral metabolism [235]. Structural complexity of microvascular networks poses significant experimental challenges on performing detailed in vivo studies on hemodynamics and obtaining reliable measurements of blood flow [236]. Thus, in recent years mathematical modeling has emerged as a powerful tool to gain insight 
into intricacies of blood flow control in complex vascular networks, and a number of detailed models have been developed [237-247].

Several factors need to be considered when performing simulations of blood flow in microvascular networks. These factors arise due to: 1) complex topological organization of dense capillary beds, 2) interactions between individual red blood cells and the inner walls of rigid vessels, and 3) the non-continuum nature of blood when flowing through narrow vessels [238-240, 248]. Red blood cells mainly flow towards the centerline of the vessel. This phenomenon, known as the Fåhræus effect, causes the formation of a cell-free layer (plasma rich) near the vessel wall and affects blood flow resistance and viscosity [249]. Experimental findings by Fåhræus and Lindqvist [250] suggested that the apparent viscosity of blood heavily depends on the diameter of the vessel. At diameters less than $300 \mu \mathrm{m}$, blood viscosity decreases with diameter, a phenomenon termed as the FåhræusLindqvist effect. This trend continues down to diameters of $\sim 6 \mu \mathrm{m}$, where the apparent viscosity reaches its minimum [242]. Below this diameter, however, apparent viscosity steeply increases. Another important observation of blood flowing through vascular networks is unequal partitioning of red blood cells at bifurcations [251], which is termed as the "phase separation" effect. This uneven distribution of red blood cells at vessel bifurcations depends on the relative diameter of daughter branches and the fraction of blood flow entering each branch.

In simulations performed in this chapter we utilized previously developed empirical models $[242,251]$, which capture the abovementioned phenomena, to study the response of the microvascular network to dilations in the feeding PA. We first examine statistical features 
of the network under study, such as the distribution of lengths, diameters, and vascular resistance. We then perform steady-state simulations of volumetric flow rate and discharge hematocrit $\left(\mathrm{H}_{\mathrm{D}}\right)$ surrounding the feeding arteriole at various levels of arteriolar dilation, as well as at different pressure gradients. Next, we analyzed the transit times of possible paths that a red blood cell can take from entering the network through the inlet of the feeding PA and exiting through a draining PV. Lastly, we performed transient simulations to examine the hemodynamic response to a time-dependent profile of dilation for the feeding arteriole.

\subsection{METHODS}

\subsubsection{Model development}

The microvascular network was represented using principles of graph theory, with vessel junctions corresponding to nodes and vessel segments corresponding to edges. The network was treated as a directed graph, where the edge directions were defined based on the direction of blood flow. The procedure used for preparing the network for simulations was as follows: 1) raw vectorized data of the upper cortex of mice were obtained from Kleinfeld, et al. [96] that provided lengths, radii, and vessel connectivity (we performed simulations on the same network used in chapters 2 and 5), 2) an in-house algorithm was developed to extract the adjacency matrix from the data, which is a square matrix of zeros and ones indicating node connectivity, 3) a directed graph was formed using the adjacency matrix, with direction of the edge indicating the direction of the flow, and 4) self-loops and stranded components were removed from the graph network. 
Volumetric flow rate in vessels was computed using the continuity equation which ensures conservation of flow at each vessel junction with the assumption that blood flow through each vessel segment follows the Hagen-Poiseuille law. This implies:

$$
\begin{aligned}
& \sum_{j}^{N} Q_{i j}=0 \\
& Q_{i j}=\frac{\left(P_{i}-P_{j}\right)}{L} \frac{\pi r^{4}}{8 \eta_{\text {vivo }}}
\end{aligned}
$$

where $\mathrm{N}$ is the number of nodes connected to the $\mathrm{i}^{\text {th }}$ node, $\mathrm{Q}_{\mathrm{ij}}$ is the volumetric flow rate, $\mathrm{L}$ is the vessel length, $r$ is the vessel radius, $\left(P_{i}-P_{j}\right)$ is the pressure drop between nodes $i$ and $\mathrm{j}$, and $\eta_{\text {vivo }}$ is the in vivo viscosity of blood:

\subsubsection{Fåhræus-Lindqvist effect}

Mathematical formulations that account for the Fåhræus-Lindqvist effect are based on previous work by Pries and coworkers [238-240]. The in vitro viscosity $\left(\eta_{\text {vitro }}\right)$ is obtained with the following equation:

$$
\eta_{\text {vitro }}=1+\left(\eta_{0.45}-1\right)\left(\frac{\left(1-\mathrm{H}_{\mathrm{D}}\right)^{\mathrm{C}}-1}{(1-0.45)^{\mathrm{C}}-1}\right)
$$

where $\eta_{0.45}$ is the apparent blood viscosity with a discharge hematocrit $\left(\mathrm{H}_{\mathrm{D}}\right)$ of 0.45 in $(\mathrm{mmHg} \cdot \mathrm{s}):$

$$
\eta_{0.45}=220 \mathrm{e}^{-1.3 \mathrm{D}}+3.2-2.44 \mathrm{e}^{-0.06 \mathrm{D}^{0.645}}
$$

and the dimensionless factor $\mathrm{C}$ is defined as:

$$
\mathrm{C}=\left(0.8+\mathrm{e}^{-0.075 \mathrm{D}}\right)\left(-1+\frac{1}{1+10^{-11} \mathrm{D}^{12}}\right)+\frac{1}{1+10^{-11} \mathrm{D}^{12}}
$$

The in vivo viscosity, $\eta_{\text {vivo }}$, is calculated according to the following equation: 


$$
\eta_{\text {vivo }}=\eta_{\text {vitro }}\left(\frac{D}{D_{\text {eff }}}\right)^{4}
$$

where $\mathrm{D}$ is the outer diameter of the vessel and $\mathrm{D}_{\text {eff }}$ is the effective vessel diameter taking into account the endothelial subsurface layer (ESL), calculated based on detailed derivations available in [248].

\subsubsection{Phase separation}

At bifurcations, red blood cells are split unequally between two daughter vessels. The fractional erythrocyte flow $\left(\mathrm{FQ}_{\mathrm{E}}\right)$ in a daughter branch is a function of the fraction of blood flow $\left(\mathrm{FQ}_{\mathrm{B}}\right)$ entering in that branch:

$$
\operatorname{logit} \mathrm{FQ}_{\mathrm{E}}=\mathrm{A}+\mathrm{B} \operatorname{logit}\left(\frac{\mathrm{FQ}_{\mathrm{B}}-\mathrm{X}_{0}}{1-2 \mathrm{X}_{0}}\right)
$$

where $\mathrm{A}, \mathrm{B}$, and $\mathrm{X}_{0}$ are parameters reported by Pries et al. [238] obtained from linear fitting of experimental data, and are given by:

$$
\begin{aligned}
& A=\frac{-6.96 \ln \frac{D_{\alpha}}{D_{\beta}}}{D_{f}} \\
& B=\frac{1+6.98\left(1-H_{D}\right)}{D_{f}} \\
& X_{0}=\frac{0.4}{D_{f}}
\end{aligned}
$$$$
\text { Equation 6-9 }
$$$$
\text { Equation 6-10 }
$$

The $\mathrm{X}_{0}$ parameter is the minimum $\mathrm{FQ}_{\mathrm{B}}$ required for erythrocytes to enter the daughter branch. If $\mathrm{FQ}_{\mathrm{B}}$ is below $\mathrm{X}_{0}$, no erythrocytes will enter the daughter vessel, making $\mathrm{FQ}_{\mathrm{E}}=$ 0. 


\subsection{4 $\quad \mathrm{N}^{\text {th }}$ Degree Junctions}

The implemented phase separation model was formulated assuming that one feeding vessel bifurcates into two daughter vessels, representing a $3^{\text {rd }}$ degree junction in graph theory terminology. In the network used for our simulations, junction degrees of greater than three exist and, therefore, require special consideration. To overcome this limitation, the phase separation model was extended by identifying three cases. The first case is when one feeding vessel splits into exactly two daughter vessels. In this case, the original phase separation model was used. The second case is where multiple feeding vessels converge into a single daughter vessel. For this scenario, blood flow entering the daughter vessels is the sum of all feeding vessel flows, given by:

$$
Q=Q_{f 1}+Q_{f 2}+\cdots Q_{f n}
$$

where $\mathrm{Q}_{\mathrm{fi}}$ is the volumetric blood flow rate in the $\mathrm{i}^{\text {th }}$ feeding vessel. The resultant diameter of the equivalent feeding vessel, $D_{f}$, is assumed to be the mean diameter of all feeding vessels and the resultant $H_{D}$ is assumed to be a weighted average of the discharge hematocrits of each feeding vessel based on their flow rates:

$$
H_{D}=\frac{Q_{f 1} H_{f 1}+Q_{f 2} H_{f 2}+\cdots Q_{f n} H_{f n}}{Q_{f 1}+Q_{f 2}+\cdots Q_{f n}}
$$

Equation 6-12

where $H_{f i}$ is the $H_{D}$ in the $i^{\text {th }}$ vessel. The third case is when there is only one feeding vessel, but more than two daughter vessels are connected to it. In this case, the $H_{D}$ of the feeding vessel is divided among the daughter vessels based on the relative diameter of each branch. 


\subsection{TRANSIENT BLOOD FLOW}

Mathematical derivations describing time-dependent changes in $\mathrm{H}_{\mathrm{D}}$ were based, with some modification, on the work outlined in [252]. Assuming the vessel segment as a straight tube with a constant diameter, the balance of the tube hematocrit $\left(\mathrm{H}_{\mathrm{T}}\right)$ can be described as:

$$
\frac{\partial \mathrm{H}_{\mathrm{T}}}{\partial \mathrm{t}}+\frac{\partial \mathrm{V}_{\mathrm{rbc}}}{\partial \xi}=0
$$

Equation 6-13

where $V_{\mathrm{rbc}}$ is the velocity of red blood cells and $\xi$ is the distance along the centerline of the vessel. Based on the Fåhræus effect, $H_{D}$ is the ratio of the velocity of red blood cells to blood velocity $\left(\mathrm{V}_{\mathrm{b}}\right)$ :

$$
\begin{gathered}
\mathrm{H}_{\mathrm{D}}=\frac{\mathrm{V}_{\mathrm{rbc}}}{\mathrm{V}_{\mathrm{b}}} \\
\mathrm{V}_{\mathrm{rbc}}=\mathrm{H}_{\mathrm{D}} \mathrm{V}_{\mathrm{b}}
\end{gathered}
$$

Equation 6-15

Assuming constant blood velocity along $\xi$ in each segment, substituting $V_{r b c}=H_{D} V_{b}$ into Equation 6-13 yields:

$$
\frac{\partial \mathrm{H}_{\mathrm{T}}}{\partial \mathrm{t}}+\mathrm{V}_{\mathrm{b}} \frac{\partial \mathrm{H}_{\mathrm{D}}}{\partial \xi}=0
$$

The ratio between $\mathrm{H}_{\mathrm{T}}$ and $\mathrm{H}_{\mathrm{D}}$ is defined with the following parametric equation developed by Pries et al [242]:

$$
\chi \equiv \frac{\mathrm{H}_{\mathrm{T}}}{\mathrm{H}_{\mathrm{D}}}=\mathrm{H}_{\mathrm{D}}+\left(1-\mathrm{H}_{\mathrm{D}}\right)\left(1+1.7 \mathrm{e}^{-0.415 \mathrm{D}}-0.6 \mathrm{e}^{-0.011 \mathrm{D}}\right)
$$

Substituting $\mathrm{H}_{\mathrm{T}}$ in Equation 6-17 with Equation 6-16, and applying chain rule to the first derivatives we obtain: 
$\chi \frac{\partial \mathrm{H}_{\mathrm{D}}}{\partial \mathrm{t}}+\mathrm{H}_{\mathrm{D}} \frac{\mathrm{d} \chi}{\mathrm{dt}}+\mathrm{V}_{\mathrm{b}} \frac{\partial \mathrm{H}_{\mathrm{D}}}{\partial \xi}=0$

Equation 6-18

The expansion of Equation 6-18 will yield the following transport equation for the evolution of $\mathrm{H}_{\mathrm{D}}$ in each segment of the vascular network:

$$
\left(\chi+H_{D} \frac{d \chi}{d H_{D}}\right) \frac{\partial H_{D}}{\partial t}+V_{b} \frac{\partial H_{D}}{\partial \xi}=0
$$

Equation 6-19 was solved numerically using a first order forward finite difference approximation for $\partial \mathrm{H}_{\mathrm{D}} / \partial \mathrm{t}$ and a first order backward finite difference approximation for $\partial \mathrm{H}_{\mathrm{D}} / \partial \xi$. The initial condition for simulations was $\mathrm{H}_{\mathrm{D}}=0.45$ for all vessels in the network. We assumed inlet node pressure of $50 \mathrm{mmHg}$ for the feeding arteriole, and 0 $\mathrm{mmHg}$ for outlet nodes of the draining venules (Figure 6-1D). To ensure convergence of the transient simulations, spatial and temporal discretization of the network vessels were chosen to meet the Courant-Friedrichs-Lewy condition [253]. In the case of Equation 6-19, the Courant Number (C) used in this model is given by:

$$
\mathrm{C}=\mathrm{V}_{\mathrm{b}} \frac{\Delta \mathrm{t}}{\Delta \mathrm{x}} \leq \mathrm{C}_{\max }
$$

where $\mathrm{C}_{\max }=1, \mathrm{~V}_{\mathrm{b}}$ is the velocity of blood, and $\Delta \mathrm{x}$ and $\Delta \mathrm{t}$ are the spatial and temporal discretization, respectively. All simulations were performed using MATLAB 2018b on a personal computer. 


\subsection{RESULTS}

\subsubsection{Statistical Properties of the Microvascular Network}

Figure 6-1 shows some relevant statistics of the network under study. Network geometry is provided in Figure 6-1A, with segments colored according to the vessel type, i.e., capillaries (grey), arterioles (red), and venules (blue). In all simulations performed in this chapter, we define a vessel as a segment starting from one junction node (node with degree higher than two) to another. Figure 6-1 B and $\mathrm{C}$ show histograms of the distribution of lengths and vessel diameters in the network. Both distributions are left-skewed, indicating a large number of capillary segments with smaller diameter and lengths compared to PAs and PVs. Diameters and lengths of vessel segments are plotted against each other (Figure 6-1 D) and colored based on vessel type. Distribution of capillary diameters is concentrated at values below $\sim 5 \mu \mathrm{m}$, while the arteriole and venule diameters exhibit greater variability. The relationship between vascular resistance with vessel diameter and length is shown in Figure 6-1 E and Figure 6-1 F. Specifically, Figure 6-1 E shows a non-linear relationship between vessel diameter and resistance with colors indicating vessels with approximately same length, and Figure 6-1 F shows that vascular resistance is linearly dependent on vessel length with colors assigned by grouping vessels by diameter. These relationships are in agreement with the Hagen-Poiseuille law (Equation 6-2). 

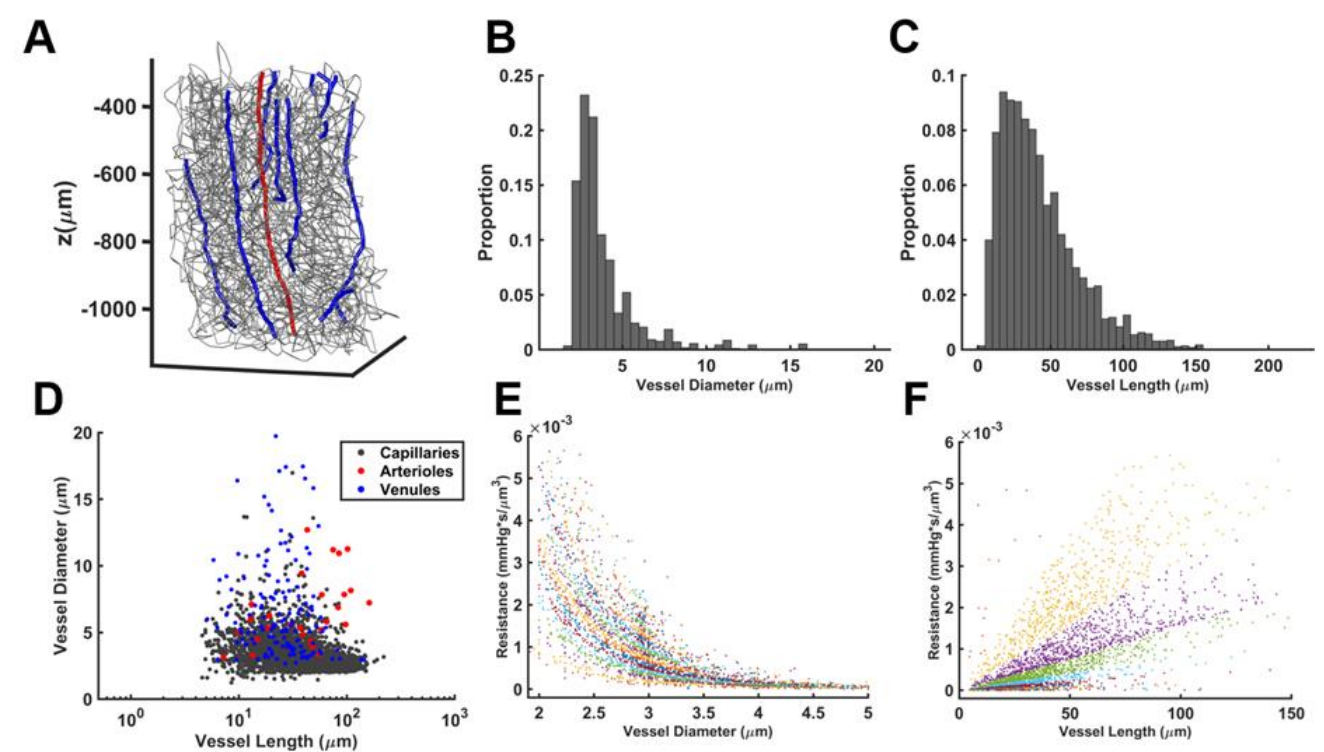

Figure 6-1 Statistical features of the microvascular network. A) microvascular network geometry with vessels colored by type: capillaries (grey), arterioles (red), venules (blue). B,C) histogram plots of the distribution of vessel lengths and diameters, respectively. The network consists of $\sim 3400$ capillary, $\sim 140$ venules, and $\sim 30$ arteriolar segments. D) scatter plot of the vessel diameters and lengths colored based on vessel type: capillaries (grey), arterioles (red), venules (blue). E) Scatter plot of the vascular resistance vs. vessel diameter with points colored based on grouping vessels by length. F) Scatter plot of the vascular resistance vs. vessel length with points colored based on grouping vessels by diameter.

\subsection{STEADY-STATE BLOOD FLOW AND DISCHARGE HEMATOCRIT}

Steady-state profiles of $\mathrm{Q}$ and $\mathrm{H}_{\mathrm{D}}$ in the microvascular network is shown in Figure 6-2. We assumed a single inlet node (red arrow) at the tip of the feeding arteriole, surrounded by draining venules with outlet nodes (white arrows located at the top) (Figure 6-2 A). Inlet and outlet pressure were assumed to be 50 and $0 \mathrm{mmHg}$, respectively. The steady state flow distribution shows highest values in the arterioles and venules (Figure 6-2 A), potentially owing to the strong dependence of vascular resistance to vessel diameter. $\mathrm{H}_{\mathrm{D}}$ was highest within the draining venule closest to the inlet node (dark red regions in Figure 6-2 B). Distributions of Q (Figure 6-2 C-E) and $\mathrm{H}_{\mathrm{D}}$ (Figure 6-2 F-H) are shown for each vessel type. $\mathrm{H}_{\mathrm{D}}$ in the capillaries (Figure 6-2 F) was widely distributed with $\sim 50 \%$ of the capillary vessels having a $\mathrm{H}_{\mathrm{D}}$ of $<0.1$, whereas distributions in the arterioles and venules 
are closer to 0.45 percent $H_{D}$. Q values between vessel types are substantially different, mainly due to the strong dependence of flow resistance to vessel length and diameter, with highest flow rates in the arterioles and lowest in the capillary segments.
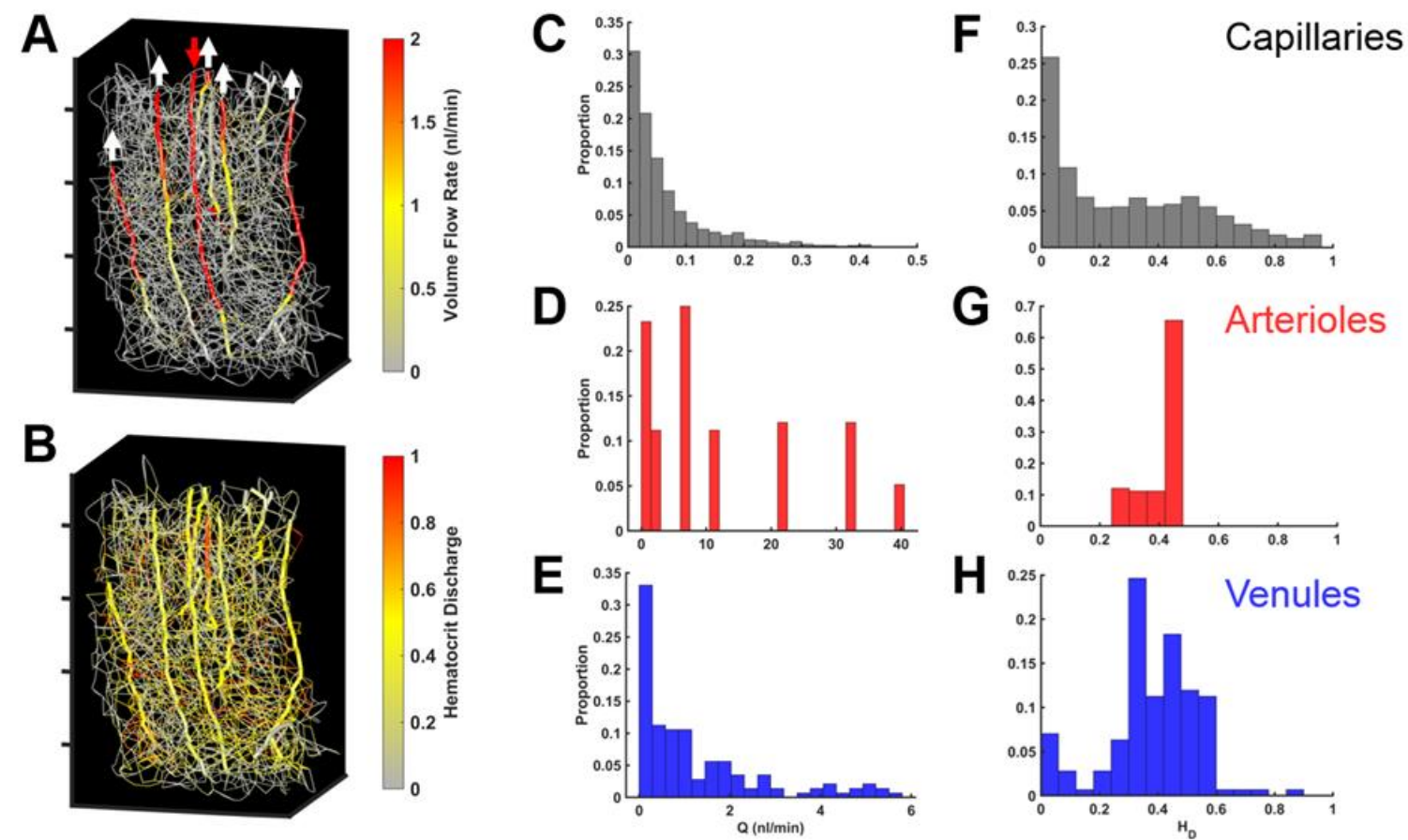

Figure 6-2 Steady state distribution of volumetric blood flow rate $(\mathrm{Q})$ and discharge hematocrit $\left(\mathrm{H}_{\mathrm{D}}\right)$. Steady state $\mathrm{Q}$ and $\mathrm{H}_{\mathrm{D}}$ simulations were performed in the microvascular network in panel $\mathrm{A}$, with red arrow indicating the inlet $(\mathrm{P}=50 \mathrm{mmHg})$ and white arrows indicating outlet nodes $(\mathrm{P}=0 \mathrm{mmHg})$. A) Steady state Q distribution color-coded on geometry B) Steady state $\mathrm{H}_{\mathrm{D}}$ distribution color-coded on geometry. C-E) Histograms showing the distributions of $\mathrm{Q}$ for each vessel type: capillaries (grey), arterioles (red), venules (blue). F-H) Histograms showing the distributions of $\mathrm{H}_{\mathrm{D}}$ for each vessel type: capillaries (grey), arterioles (red), venules (blue).

\subsubsection{Effect of Arteriole Dilation and inlet pressure on network hemodynamics}

In Figure 6-3, we provide the steady state response of the system to various levels of dilation in the feeding PA with the constant inlet pressure. To quantify this effect, we placed hypothetical concentric cylinders with increasing diameters around the feeding arteriole and computed mean and standard deviation of $\mathrm{Q}$ within each cylinder and reported the results for capillaries in Figure 6-3 A and for the larger diameter arterioles and venules 
in Figure 6-3 B. Both panels show decline of mean blood flow in the vasculature with increasing distance from the feeding arteriole. At each specific distance, increasing the dilation level elevates the mean blood flow in the network. The percent change in Q within the network is shown at four levels of arteriolar dilation color-coded on the geometry (Figure 6-3 C-F). Dilation of 0\% represents the baseline corresponding to the steady-state Q profile shown in Figure 6-2 A. Results exhibit a trend of increasing flow rate as the dilation percentage increases. Interestingly, in these simulations dilation of the feeding arteriole resulted in higher levels of flow rate within the deeper layers of the network.
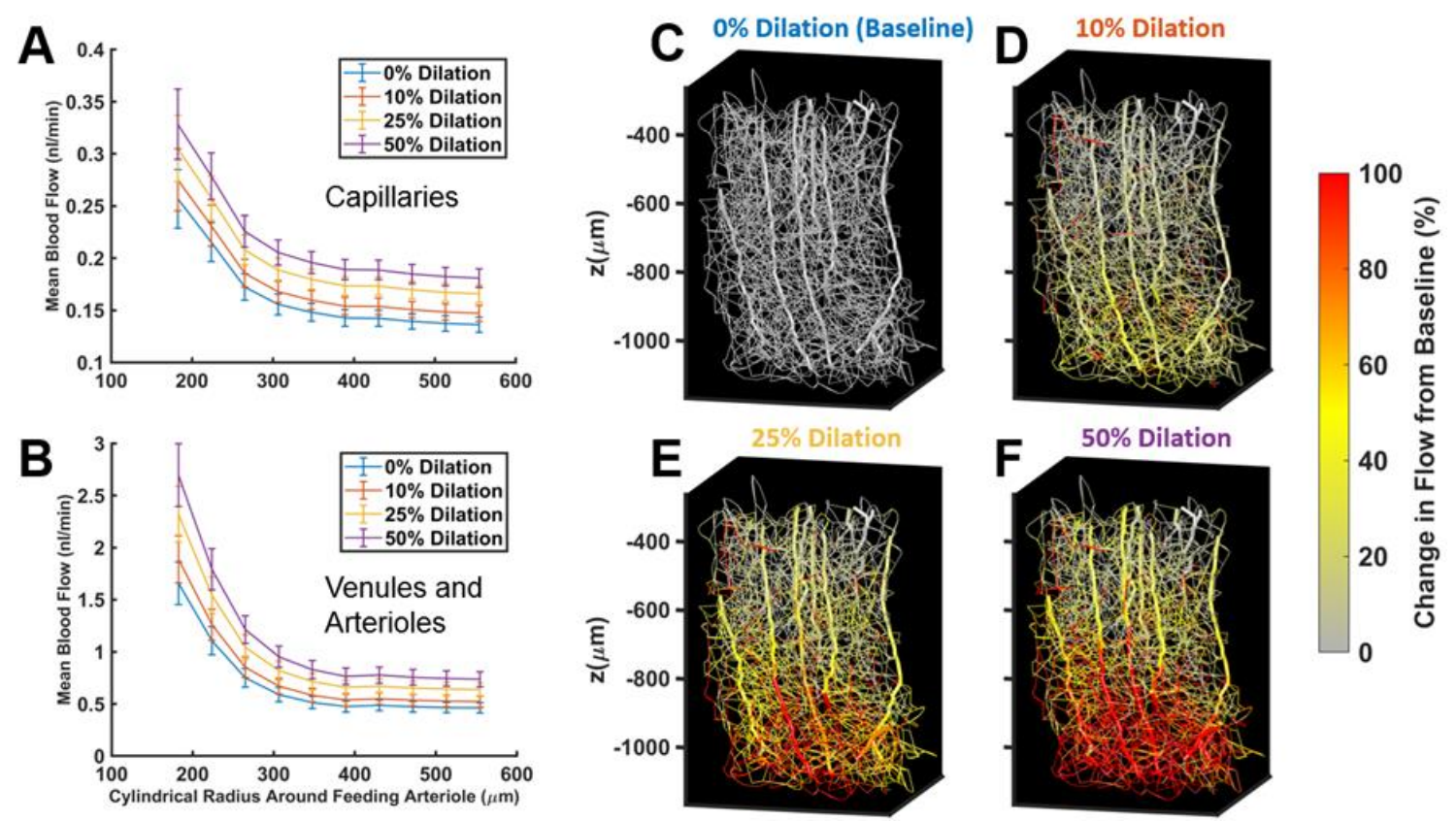

Figure 6-3- Effect of parenchymal arteriole dilation on the steady-state flow distribution in the network. Steady-state flow simulations were performed at four levels of feeding arteriole dilation $(0,10,25,50 \%)$. With each dilation percentage used in the simulations, the mean and standard deviation of the blood flow rate was computed within concentric cylindrical regions around the feeding parenchymal arterioles. Results are shown for flow rate in capillaries (A) and combined flow of the arterioles and venules (B). C-F) The microvascular is shown colored based on percent change in flow from the baseline, with baseline corresponding to the profile shown in Figure 6-2A, for 0, 10, 25 and 50\% dilation in the feeding PA.

Mean flow rates were also calculated at various inlet pressures (Figure 6-4) with a constant diameter of the feeding arteriole (panel A for capillaries, and panel B for arteriole and 
venules). Similar patterns to results in Figure 6-3 were also observed in these simulations. Increasing the inlet pressure increased the mean $Q$ in the network, showing a decreasing pattern with increasing distance from the feeding arteriole. It is noteworthy that changes in the mean $\mathrm{Q}$ in response to pressure gradients used in these simulations were higher that the differences shown in Figure 6-3 resulting from dilation of the PA.
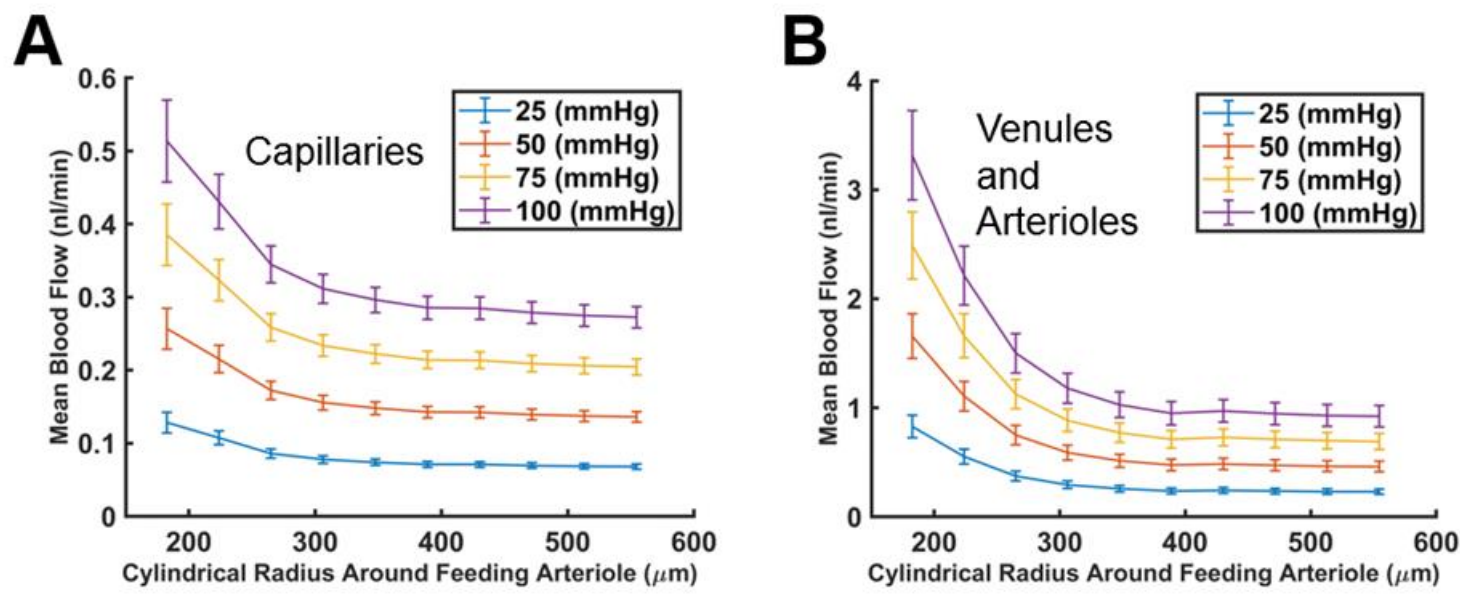

Figure 6-4- Effect of increasing parenchymal arteriole inlet pressure on the steady-state flow distribution in the network. Mean and standard deviation of steady state volumetric blood flow is shown for capillaries (A) and PAs and PVs (B) in concentric cylindrical regions with increasing diameter around the feeding PA, for 25, 50, 75, and $100 \mathrm{mmHg}$ of PA inlet pressure.

\subsection{EFFECT OF THE EXTENT OF UPSTREAM VASODILATION ON NETWORK HEMODYNAMICS}

As discussed in CHAPTER 2, neuronally-induced local hyperpolarization initiated from deep layers of the cortex might passively propagate along the PAs and attenuate with distance (Figure 2-6). We also provided the potential existence of regenerative mechanisms of signal conduction where the hyperpolarizing signal can be conducted over long distances along the PA with minimal or no loss. Thus, it is important to investigate how the length constant of the spread of vasodilatory signals, or equivalently the spatial extent of arteriolar 
dilation, can change the hemodynamic response of the network. To evaluate this effect, we assumed maximal dilation of the feeding arteriole at the bottom of the network $(50 \%$ increase in the diameter) which tapers off exponentially upstream the PA with a given length constant $(\lambda)$.

Figure 6-5 demonstrates the dependency of the percentage change in the mean volume flow rate in the network to the length constant of arteriolar dilation at a constant inlet pressure $(50 \mathrm{mmHg}$ ) for all simulations. Interestingly, mean flow in the system shows a strong dependence to $\lambda$, showing a sigmoidal relationship (the red fitted curve). This dependence might signify the importance of potential regenerative-like mechanisms which allows the spread of the hyperpolarizing signals over long distances towards surface pial arteries and subsequently result in higher changes in $\mathrm{Q}$ in the tissue region.

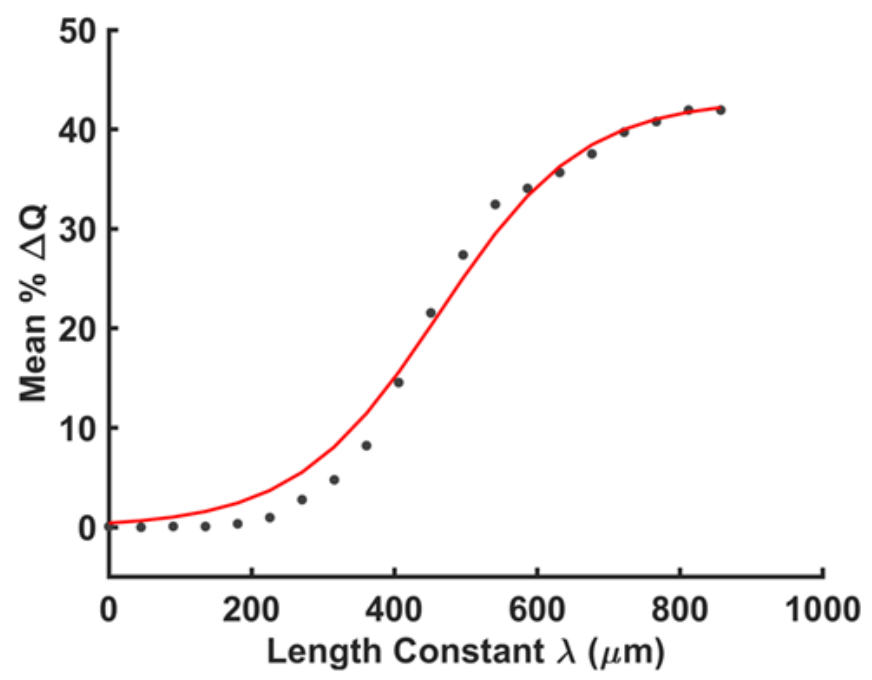

Figure 6-5 Effect of the extent of arteriolar dilation on the mean percentage change of blood flow in the network. Steady state flow simulations were performed on the network shown in Figure 6-2A, where the PA was dilated $50 \%$ at the bottom of the network and the dilation tapered off with a length constant of $\lambda$ along the PA. For all simulations the inlet pressure was assumed to be $50 \mathrm{mmHg}$. The resultant percent change in the mean volumetric flow rate in the network is plotted against varying $\lambda$ values (black dots). Results are fitted using a sigmoidal fit (red line: $\mathrm{f}(\mathrm{x})=0.4 /(0.01+\exp (-\mathrm{x}))$, with $\left.\mathrm{R}^{2}=0.98\right)$ 


\subsection{TRANSIT TIMES OF ERYTHROCYTES IN MICROVASCULAR NETWORKS}

Heterogeneity in microvascular network structures greatly contributes to transit times of red blood cells along the microvasculature. In the network used in this chapter, we define transit time as the time it takes for a red blood cell entering the inlet (from the feeding PA) to exit from one of the outlets (tip of the venules). We developed an algorithm to track the movement of a red blood cell flowing in the network, with red blood cell flow rate $\left(Q_{\mathrm{rbc}}=\right.$ $\mathrm{QH}_{\mathrm{D}}$ ) of each branch over $\mathrm{Q}_{\mathrm{rbc}}$ of the feeding vessel as the probability of the red blood cell entering the branch. Consequently, if the feeding vessel is connected to only one branch, the red blood cell is forced to flow through the connecting vessel. At vessel bifurcations, however, the red blood cell will enter one of the daughter vessels based on the aforementioned probability distribution.

Using this algorithm, we simulated 10,000 paths a red blood cell would take in the network, and showed the distribution of the corresponding transit times in Figure 6-6 A. Figure 6-6 B shows the cumulative proportion of transit times shown in Figure 6-6A, indicating that in almost $80 \%$ of cases the red blood cell would be flushed out of the system in a matter of few seconds. Figure 6-6 C illustrates the strong positive correlation of the total transit time to the number of bifurcations a red blood cell flew through along its path (Pearson correlation coefficient $=0.71$ (the red line), p-value $=4 \times 10^{-154}$ ). Figure 6-6 D shows the distribution of total number of bifurcations, and capillary generations for all simulated paths. On average, red blood cells passed through $\sim 10$ capillary generations before leaving the system from one of the outlets of the network. Some example paths and their 
corresponding transit times are shown in (Figure 6-6 E-H), with arrows indicating inlet (red) and outlet (blue) of the network.
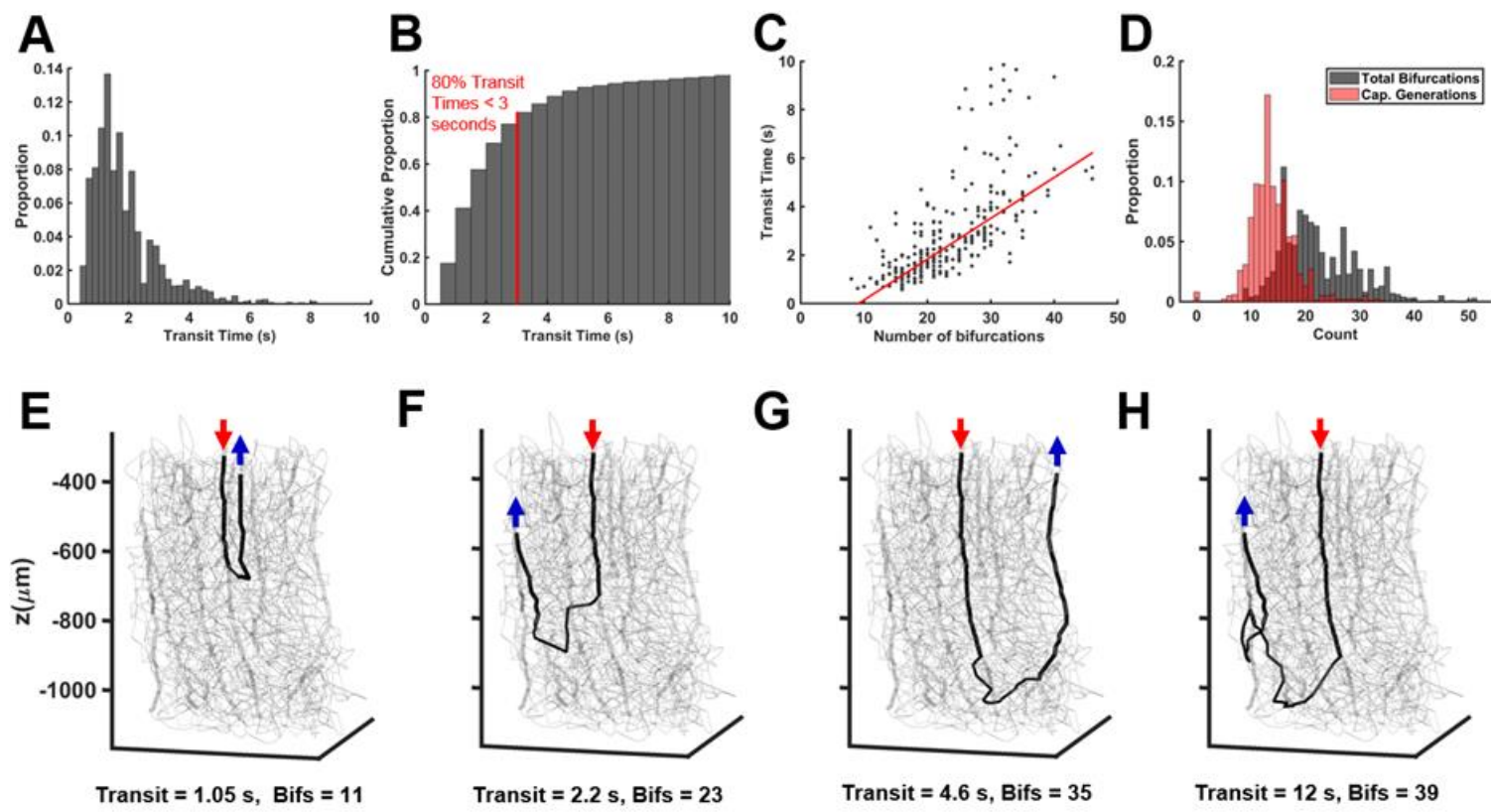

Figure 6-6- Red blood cell transit times. An algorithm was developed to track the path of a red blood cell from the inlet of the network to any of the outlets (refer to Figure 6-2 for positions of inlets and outlets). At each bifurcation the red blood cell randomly chooses a daughter branch over another according to the probability based on relative $\mathrm{Q}_{\mathrm{rbc}}$. 10,000 potential paths were generated, and their distributions are shown in panel (A). Panel (B) shows the cumulative proportion distribution of the resident times of paths generated in (A). C) Path transit times as a function of number of bifurcations along the simulated paths of red blood cells in (A) and (B). The red line shows positive correlation of transit times to number of bifurcations (Pearson correlation coefficient $=0.71$ (the red line), $\mathrm{p}$-value $=4 \times 10^{-154}$ ). D) histogram of the distribution of total bifurcations (gray) and number of capillary generations from 10,000 simulated paths of red blood cells. Panels (E-F) show example simulated paths with red arrow showing the inlet and blue arrow showing the outlet. The respective transit time and the number of bifurcations along the path is shown below the graphs.

\subsection{TRANSIENT BLOOD FLOW IN RESPONSE TO ARTERIOLAR}

\section{DILATION}

A transient profile of dilation with a time constant of one second for both increase and

decrease of the diameter is assumed for transient flow simulations (Figure 6-7 A). This profile was chosen to mimic the arteriolar dilations observed in the ex vivo Cap-PA preparations in $[30]$ in response to local increases in $\left[\mathrm{K}^{+}\right]_{\mathrm{o}}$ around capillaries. The 
numerical procedure outlined in the methods section was utilized to compute the evolution of $\mathrm{H}_{\mathrm{D}}$ and $\mathrm{Q}$ over time. Figure 6-7 B shows five different time instances of the network colored based on percent change from the flow rates prior to dilation (Figure 6-2A). Time frames show the change in flow rate at rest ( 2 seconds), during the rise in diameter (5 seconds), the plateau phase (12 seconds), recline in diameter (15 seconds), and return to the original diameter (20 seconds). Results show how flow distribution in the network would evolve upon the temporal changes in the PA diameter. Similar to steady-state results shown in Figure 6-3, the increase in flow was more concentrated in the lower regions of the network. Also, comparison of the plateau phase of the diameter increase (at 12 seconds) during transient simulations with the equivalent steady state profile of Figure 6-3 F suggests that the flow distribution reaches its steady state within few seconds of the stabilization in the arteriolar diameter. This finding corroborates the observations in Figure 6-6 that most simulated random paths of red blood cells along the network had a total transit time in the order of few seconds (the time required for red blood cells to be flushed out of the system). The validity of these observations, however, remains to be tested in experimental studies. 


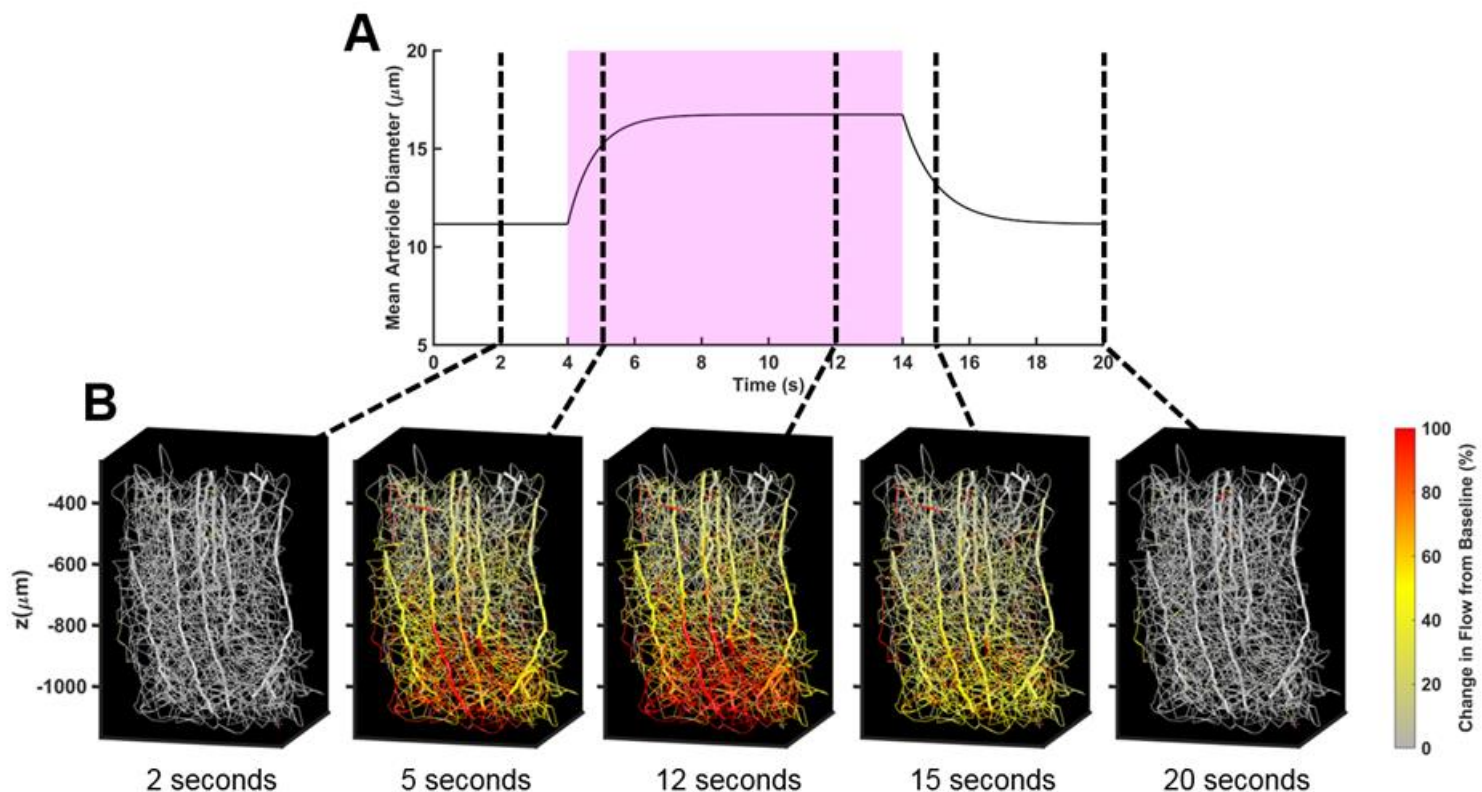

Figure 6-7 - Transient simulations of blood flow in the microvascular network. The transient blood flow of the same vascular network of Figure 6-2 A is simulated assuming the PA diameter follows the pattern shown in panel (A). The inlet pressure is assumed to be $50 \mathrm{mmHg}$. Panel (B) shows color-coded changes in the flow distribution with respect to baseline ( 2 seconds), during the rise ( 5 seconds), plateau (12 seconds), recline (15 seconds), and upon return to baseline (20 seconds).

\subsection{DISCUSSION}

The primary aim of this chapter was to relate NVC mechanisms discussed earlier with the distribution of blood flow and hematocrit to different regions of brain microvasculature. As discussed in great depth in previous chapters, changes in $\mathrm{V}_{\mathrm{m}}$ of PA SMCs is the major determinant of the myogenic tone and the diameter of these vessels. We studied how hyperpolarizing signals can be initiated from various locations along the brain vasculature and identified molecular determinants of the extent of the conducted responses to upstream PAs. We also identified how pathological conditions including small vessel disease and cortical spreading depression can significantly affect the resting $\mathrm{V}_{\mathrm{m}}$, and the vascular response to neuronally-induced stimuli. Although at the current stage our flow models are not directly coupled to models of cell electrophysiology in previous chapters, here we 
analyzed the effect of a varying levels of PA dilation and pressures on blood flow and hematocrit distribution in the network. This modeling platform serves as our initial effort in providing a multiscale mathematical modeling framework for $\mathrm{NVC}$ where macroscopic tissue-level responses of the system under normal or pathological conditions can be related to microscopic cell-level electrophysiological activity.

\subsubsection{Flow pattern in response to increases in PA diameter and inlet pressure}

In both steady state (Figure 6-3) and transient (Figure 6-7) simulations, arteriolar dilation was shown to increase the blood flow in the microvascular network under study. Similar results, although more pronounced, was observed when the inlet pressure was increased under a constant diameter (Figure 6-4). This increase in flow attenuated with distance from the feeding arteriole, indicating the reduction of volumetric flow rate at the bifurcations of smaller diameter capillary segments as well as the reduction of pressure gradient farther away from the PA. A notable feature illustrated in simulations is the higher flow rates towards the lower layers of the network; rather than a uniform increase all throughout the depth of the network. This result might be due to: 1) an uneven distribution of capillaries along the depth of the network, 2) higher numbers of branch points at the lower portions of the PA arteriole, or 3) the downward direction of flow resulting from the assumed boundary conditions (Figure 6-2 A). One has to bear in mind that these potential explanations are all related to the structural nature of the specific network under study, and a decisive conclusion can only be made upon a large number of simulations on a variety of vascular structures. 


\subsubsection{Distributions of Hematocrit and Plasma due to Phase Separation Laws}

As can be observed in Figure 6-2 F, a wide distribution of $\mathrm{H}_{\mathrm{D}}$ is predicted in the dense capillary network, potentially due to the difference in the diameter of capillary segments at bifurcations according to phase separation principles. An important consequence of this phase separation effect is the high number of low-hematocrit capillary regions (Figure 6-2 B and Figure 6-2 F), which may lead to the presence of hypoxic regions in the tissue surrounding it. It is important to note that this predicted distribution of $\mathrm{H}_{\mathrm{D}}$ can also be attributed to the equations used for capturing phase separation. For instance, recently, Gould et al. [237] provided a comparative study on mathematical models of blood flow control in large vascular networks and demonstrated that with some modifications in these equations, more uniform distributions of $\mathrm{H}_{\mathrm{D}}$ and oxygen transport can be predicted. Although authors claim that distributions obtained from their model are closer to experimental recordings compared to those of widely used Pries and Secomb models [238240], the validity of the these propositions need to be tested in more comprehensive experimental studies.

\subsubsection{Limitations and future directions}

As shown in simulations performed in this chapter, the structural complexity of microvascular networks greatly influences the spatiotemporal characteristics of observed hemodynamic responses. For this reason, studying a single unique network is inadequate if the goal is to gain a robust and widely applicable understanding of the hemodynamic response. Also, structural properties may greatly vary among different animal species, as well as within different regions of the brain. Therefore, simulation results in this chapter 
may not necessarily serve as a global representation of the hemodynamic response during NVC. Nevertheless, the platform provided in this chapter can be utilized to study microvascular networks from different species upon availability of experimental data.

Due to high technological requirements necessary for obtaining detailed microvascular structures with high resolution, a number of groups have recently resorted to statistical approaches, primarily based on principles of Voronoi algorithms, to simulate large network structures possessing the same statistical properties as one found in different species [254257]. The combination of detailed mathematical models, like the one developed in this chapter, with these approaches can yield extremely useful information in advancing our knowledge of the hemodynamic response during NVC.

Another important factor that can greatly change the distribution of blood flow within brain vascular networks during NVC is the activity of pericytes. The unique structure of these cell types, i.e., long processes wrapped around capillaries at vessel bifurcations, allow them to preferentially restrict blood flow into one branch and redistribute red blood cells to regions with high metabolic demands [6]. These cell types are shown to be actively involved during NVC in normally functioning brain and also in its disruption under pathologies $[6,17]$. Thus, more detailed mathematical models can be developed in the future where the electrophysiological signaling in different cell types involved in NVC, is coupled with the flow and hemodynamic models presented in this study.

Simulations performed in this chapter aim to investigate the dilatory and pressure requirements for physiologically relevant increases in the blood flow in the microvasculature. Model simulations indicated that to observe relevant increases in blood 
perfusion in response to a stimulation deep within the cortex, the vasodilatory signal must propagate several hundred microns upstream the PAs (Fig. 6-5). Robust expression of Kir2.1 channels in the microvascular endothelium allow transmitting dilatory signals over such distances (CHAPTER 2) by enabling regenerative, long-range conduction of electrical signals. Thus, neuronally-induced hyperpolarizing (vasodilatory) signals initiated at the capillary level can reach and propagate into upstream arterioles at distances that can induce significant increases in local blood supply. Predicted blood flow distribution in a geometrically accurate microvascular network highlight the importance of endothelial-mediated signaling for the regulation of the supply of blood and nutrients to regions of high metabolic demand and corroborate the physiological relevance of electrical responses in CHAPTER 2. 


\section{CHAPTER 7 CONCLUDING REMARKS}

Proper brain function heavily relies on an in-time delivery of nutrients and oxygen in response to elevations in neuronal activity. In a normally functioning brain, this momentto-moment communication between neurons and vasculature, termed as functional hyperemia or neurovascular coupling (NVC) [3], is achieved through an organized activity of a wide range of inter- and intra-cellular mechanisms, collectively known as a neurovascular unit (NVU) [4]. Disruption of NVC, in response to dysfunction of any or a combination of the components of a NVU, has been observed in a variety of neurological disorders including Alzheimer's disease, stroke, hypertension, dementia, and cortical spreading depression [5-9].

The work presented in this dissertation provided a novel multiscale mathematical modeling platform for NVC and cerebral blood flow (CBF) control under normal and diseased states with the major focus on the role of $\mathrm{K}^{+}$ions in this process. In CHAPTER 2, we investigated how capillary endothelial cells (cECs) can sense local activity of neurons and translate this activity into a retrograde vasodilatory signal that can propagate to upstream parenchymal arterioles (PAs) and regulate the dynamics of $\mathrm{CBF}$. We identified inward rectifying potassium (Kir) channels expressed in cECs as the molecular cornerstone of this capillarymediated NVC. We postulated that high expression of these channels in cECs can promote a regenerative mode of conduction of electrical signals that can transmit neuronallyinduced local hyperpolarization over long distances with minimal or no loss. 
In CHAPTER 3, our mathematical models predicted the key role of up- and downregulation of voltage-gated potassium (Kv) channels in the disruption of $\mathrm{NVC}$ in animal models with small vessel disease (SVD). We identified a "tug-of-war" dynamic between Kir and $\mathrm{Kv}$ channels in determining the membrane potential $\left(\mathrm{V}_{\mathrm{m}}\right)$ and myogenic tone of PA smooth muscle cells (SMCs) in response to NVC-associated modest elevations of $\left[\mathrm{K}^{+}\right]_{\mathrm{o}}$. Consistent with experimental data, our modeling studies predicted that increased $\mathrm{Kv}$ channel densities in CADASIL animal models would result in a more hyperpolarized resting $\mathrm{V}_{\mathrm{m}}$, and lower myogenic tone compared to control animals; while, conversely, decreased $\mathrm{Kv}$ levels in SAH would depolarize resting $\mathrm{V}_{\mathrm{m}}$ and increase the myogenic tone of PA SMCs.

CHAPTER 4 and CHAPTER 5 focused on understanding the effect of unphysiological elevations in $\left[\mathrm{K}^{+}\right]_{\mathrm{o}}$ during cortical spreading depression on the response of brain vasculature during this phenomenon. Using our newly developed current source density analysis method, presented in CHAPTER 4, for the first time, we provided an estimate of transmembrane current sources during cortical spreading depression in rats. Using this estimate as an indicator of the extent and duration of the cortical depression wavefront, in CHAPTER 5 we provided a potential mechanism underlying the observed multiphasic responses in vasculature during this phenomenon [53]. Our model simulations predicted that the nonlinearities in the current-voltage relationship of Kir channels result in the depolarization of vascular cells at the wavefront (at high levels of $\left[\mathrm{K}^{+}\right]_{\mathrm{o}}$ ), while before and after the wave (during the decay of $\left[\mathrm{K}^{+}\right]_{0}$ ), these channels conduct a hyperpolarizing signal along the vessel 
Lastly, in CHAPTER 6, we provided a detailed mathematical modeling framework to observe how $\mathrm{V}_{\mathrm{m}}$ - dependent changes in the PA diameter, affects the distribution of blood flow and hematocrit along the vessels in a large interconnected brain vascular network. This model, along with the electrophysiological models developed in previous chapters provide a theoretical platform where the effect of neuronally-induced stimuli at different locations along the brain vasculature and their corresponding changes in CBF can be studied in normal and diseased conditions.

Overall, simulations performed in this dissertation identified the subcellular requirements of the microvasculature to sense elevated neuronal activity in deep regions of the brain, through the increase in $\left[\mathrm{K}^{+}\right]_{\mathrm{o}}$, and transmit vasodilatory signals towards PAs and surface vessels to increase $\mathrm{CBF}$. We demonstrated how Kir channels expressed in capillary endothelial cells may give rise to an action potential-like mode of signal conduction along the microvasculature, a finding that can have significant implications on the general understanding of NVC and potential bottom-up signaling pathways in this process. We demonstrated how dynamics of Kir channels, and their interplay with other major ionic channels in the membrane, can provide a mechanistic explanation of the response of the vasculature under pathological conditions, in particular small vessel disease and cortical spreading depression.

\subsection{FUTURE DIRECTIONS}

\subsubsection{Mathematical Modeling}

Several aspects of this modeling framework can be expanded in the future. For instance, here we primarily focused on the role of $\mathrm{K}^{+}$ions in NVC under healthy and diseased 
conditions. However, as discussed in previous chapters, NVC involves a variety of other known mediators such as AA metabolites, PGs, and NO [17]. Therefore, an avenue for further exploration is the inclusion of detailed mathematical models of cell electrophysiology, i.e., cECs (upon new available experimental data on the characterization of ion channels and intracellular dynamics), ECs and SMCs of PAs, and accounting for the effect of these vasoactive agents on their respective target channels. For example, as has been shown in previous modeling studies, the effect of $\mathrm{NO}$ as an important vasodilator can be accounted by its direct influence on PA SMC large conductance potassium (BKCa), sodium-calcium exchanger ( $\mathrm{NCX}$ ), calcium-activated chloride $(\mathrm{ClCa})$, and sodiumpotassium-chloride $(\mathrm{NaKCl})$ cotransporter channels [258] . These models can be extremely useful in analyzing the relative contributions of parallel pathways involved in NVC, as well as the potential direct or indirect influences they might have on one another. One has to bear in mind, however, that even though these inclusions might provide important insights into understanding $\mathrm{NVC}$, increasing the level of complexity of models can also impede meaningful interpretations of model predictions. Thus, model expansion should be done with care, and should mainly be based on the particular mechanism under study.

In our current model, the levels of dilation in the PA during NVC is determined based on observed values reported in experimental studies in literature. Also, there is currently no feedback mechanism between our cell electrophysiology and blood flow models. As shown in previous studies for other vascular beds, the vessel biomechanics and the diameter can be directly modeled based on the $\mathrm{Ca}^{2+}$ dynamics of SMCs and the activity of actin-myosin cross-bridge cycle $[259,260]$. Also, it is well-established in literature that flow-induced 
changes in the transluminal pressure can feedback on PA SMC Ca ${ }^{2+}$ dynamics through the activation of non-selective cation (NSC) channels [261, 262], which can have important consequences in the regulation of vessel tone. Therefore, an important aspect to the be added to the current model is the incorporation of detailed $\mathrm{Ca}^{2+}$ dynamics in the PA SMCs, and inclusion of the biomechanics model of the vessel wall relating the flow-related transluminal pressure to $\mathrm{Ca}^{2+}$ in these cells.

Another future direction for the current modeling framework is the inclusion of a model of oxygen transport along the microvascular network and inside the tissue region containing it. This addition to the model will provide a comprehensive mathematical modeling base where the effect of microscopic, cell-level signaling pathways on macroscopic changes in tissue perfusion and oxygenation can be analyzed. Several modeling groups have used finite element (FEM) and finite difference (FDM) methods to simulate dynamics of oxygen transport inside blood vessels and in the surrounding tissue in complex structures [263267]. More recently, Secomb and colleagues also provided less computationally expensive approaches based on analytical solutions to oxygen diffusion equation using principals of Green's functions, where vessel segments are treated as point sources and tissue consumption is modeled as sinks of oxygen [268-270]. Using the combination of these approaches, and models developed in this dissertation, a wide variety of phenomena can be studies in brain vasculature. Vascular oxygen responses can also be translated into blood oxygen level dependent (BOLD) signals, which provide a theoretical paradigm for bottomup modeling of functional magnetic resonance (fMRI) data. 


\subsubsection{Proposed experimental studies}

Mathematical models developed in this dissertation provided interesting predictions on the underlying mechanisms of NVC that can be subjects of several future experimental studies. For example, simulations suggest that expression of Kir2.1 channels in cECs can result in bistability of membrane potential $\left(\mathrm{V}_{\mathrm{m}}\right)$ in response to elevations in $\left[\mathrm{K}^{+}\right]_{\mathrm{o}}$, i.e., the ability of single cECs to exist in two distinct membrane potential values (one at more depolarized potentials, and the other at more hyperpolarized voltages). This theoretical prediction can be tested experimentally by recording $\mathrm{V}_{\mathrm{m}}$ of single isolated cECs using microelectrodes during step increases of the bath potassium concentration from resting levels $(\sim 3 \mathrm{mM})$ to levels reached during $\mathrm{NVC}(\sim 10 \mathrm{mM})$. Based on the theoretical analysis presented in CHAPTER 2, the prediction would be a gradual hyperpolarization of single cECs before a massive jump of the membrane potential to more hyperpolarized potentials near the Nernst equilibrium potential of potassium ions $\left(\mathrm{E}_{\mathrm{K}}\right)$. Additionally, simulation results predicted the presence of a hysteresis in the response of single cells to step changes in $\left[\mathrm{K}^{+}\right]$(Fig. 2-2A). If this prediction is true, the transition of single cECs from hyperpolarized to depolarized $\mathrm{V}_{\mathrm{m}}$ during step decreases in bath potassium concentration will happen at a different $[\mathrm{K}+]$ compared to the reverse direction. The transition points will indicate the $\left[\mathrm{K}^{+}\right]$window for bistability.

Another important observation of the models presented in this dissertation is the existence of an action potential-like mode of conduction of hyperpolarizing signals along cECs to upstream PAs, owing to the presence of strong Kir channels in these cells. Several experimental studies can be conducted to test the validity and extent of this model 
prediction. For instance, experiments can be performed on brain slice preparations where electrical signals are initiated at the capillary site and the conduction of the signal can be tracked along the capillary and PA segments. Signal initiation at the capillary end can be done via picospritzing a $\mathrm{K}^{+}$ionophore, e.g. valinomycin, or current injection with microelectrodes, to allow hyperpolarization of cells even in the absence of Kir channels, i.e., in $\mathrm{Kir}^{-/-}$mice. Another method for initiation of signals in cECs is to use focal light stimulation in slices obtained from optogenetically-enabled mice, where only cECs can be stimulated. Optogenetics is an avant-garde approach which genetically targets specific cell types with high degree of specificity and high levels of spatial and temporal resolution [271]. The resultant changes in the $V_{m}$ along capillaries and arterioles can be monitored either using microelectrodes at different locations along the PAs, or via imaging the changes in the fluoresce level of voltage sensitive dyes (VSDs), e.g. Di-8-ANEPPS. Based on the prediction from our mathematical models, an "all-or-none" response in PAs is predicted when stimulation of capillary increases above a threshold value. Additionally, we expect the loss of this regenerative signaling upon reduction of the activity of Kir channels, either chemically via application of $\mathrm{Ba}^{2+}$ or PGE2, or through genetically knocking out of Kir channels. 


\section{REFERENCES}

[1] C.S. von Bartheld, J. Bahney, S. Herculano-Houzel, The search for true numbers of neurons and glial cells in the human brain: A review of 150 years of cell counting, Journal of Comparative Neurology, 524 (2016) 3865-3895.

[2] A.Y. Shih, C. Rühlmann, P. Blinder, A. Devor, P.J. Drew, B. Friedman, P.M.

Knutsen, P.D. Lyden, C. Mateo, L. Mellander, Robust and fragile aspects of cortical blood flow in relation to the underlying angioarchitecture, Microcirculation, 22 (2015) 204-218.

[3] C.S. Roy, C.S. Sherrington, On the regulation of the blood-supply of the brain, The Journal of physiology, 11 (1890) 85.

[4] V. Muoio, P. Persson, M. Sendeski, The neurovascular unit-concept review, Acta physiologica, 210 (2014) 790-798.

[5] H. Girouard, C. Iadecola, Neurovascular coupling in the normal brain and in hypertension, stroke, and Alzheimer disease, Journal of applied physiology, 100 (2006) 328-335.

[6] N.B. Hamilton, D. Attwell, C.N. Hall, Pericyte-mediated regulation of capillary diameter: a component of neurovascular coupling in health and disease, Frontiers in neuroenergetics, 2 (2010) 5.

[7] C. Iadecola, The pathobiology of vascular dementia, Neuron, 80 (2013) 844-866.

[8] A.A. Phillips, F.H. Chan, M.M.Z. Zheng, A.V. Krassioukov, P.N. Ainslie, Neurovascular coupling in humans: Physiology, methodological advances and clinical implications, Journal of Cerebral Blood Flow \& Metabolism, 36 (2016) 647-664.

[9] M. Guoiu, S. Sheth, M. Nemoto, M. Walker, N. Pouratian, A. Ba, A.W. Toga, Cortical spreading depression produces long-term disruption of activity-related changes in cerebral blood volume and neurovascular coupling, Journal of biomedical optics, 10 (2005) 011004.

[10] J. Hugo, M. Ganguli, Dementia and cognitive impairment: epidemiology, diagnosis, and treatment, Clin Geriatr Med, 30 (2014) 421-442.

[11] M. Prince, R. Bryce, E. Albanese, A. Wimo, W. Ribeiro, C.P. Ferri, The global prevalence of dementia: a systematic review and metaanalysis, Alzheimers Dement, 9 (2013) 63-75 e62.

[12] M. Wortmann, Dementia: a global health priority-highlights from an ADI and World Health Organization report, Alzheimer's research \& therapy, 4 (2012) 40.

[13] E.C. Leritz, R.E. McGlinchey, I. Kellison, J.L. Rudolph, W.P. Milberg, Cardiovascular Disease Risk Factors and Cognition in the Elderly, Curr Cardiovasc Risk Rep, 5 (2011) 407-412. 
[14] M. Kivipelto, E.L. Helkala, M.P. Laakso, T. Hanninen, M. Hallikainen, K. Alhainen, S. Iivonen, A. Mannermaa, J. Tuomilehto, A. Nissinen, H. Soininen, Apolipoprotein E epsilon4 allele, elevated midlife total cholesterol level, and high midlife systolic blood pressure are independent risk factors for late-life Alzheimer disease, Ann Intern Med, 137 (2002) 149-155.

[15] L. Pantoni, Cerebral small vessel disease: from pathogenesis and clinical characteristics to therapeutic challenges, Lancet Neurol, 9 (2010) 689-701.

[16] C. Iadecola, The pathobiology of vascular dementia, Neuron, 80 (2013) 844-866.

[17] D. Attwell, A.M. Buchan, S. Charpak, M. Lauritzen, B.A. MacVicar, E.A. Newman, Glial and neuronal control of brain blood flow, Nature, 468 (2010) 232.

[18] B. Cauli, X.-K. Tong, A. Rancillac, N. Serluca, B. Lambolez, J. Rossier, E. Hamel, Cortical GABA interneurons in neurovascular coupling: relays for subcortical vasoactive pathways, Journal of Neuroscience, 24 (2004) 8940-8949.

[19] B.R. Chen, M.G. Kozberg, M.B. Bouchard, M.A. Shaik, E.M. Hillman, A critical role for the vascular endothelium in functional neurovascular coupling in the brain, Journal of the American Heart Association, 3 (2014) e000787.

[20] F. Fernández-Klett, N. Offenhauser, U. Dirnagl, J. Priller, U. Lindauer, Pericytes in capillaries are contractile in vivo, but arterioles mediate functional hyperemia in the mouse brain, Proceedings of the National Academy of Sciences, 107 (2010) 2229022295.

[21] C. Lecrux, X. Toussay, A. Kocharyan, P. Fernandes, S. Neupane, M. Lévesque, F. Plaisier, A. Shmuel, B. Cauli, E. Hamel, Pyramidal neurons are "neurogenic hubs" in the neurovascular coupling response to whisker stimulation, Journal of Neuroscience, 31 (2011) 9836-9847.

[22] B.L. Lind, A.R. Brazhe, S.B. Jessen, F.C. Tan, M.J. Lauritzen, Rapid stimulusevoked astrocyte $\mathrm{Ca} 2+$ elevations and hemodynamic responses in mouse somatosensory cortex in vivo, Proceedings of the National Academy of Sciences, 110 (2013) E4678E4687.

[23] T. Takano, G.-F. Tian, W. Peng, N. Lou, W. Libionka, X. Han, M. Nedergaard, Astrocyte-mediated control of cerebral blood flow, Nature neuroscience, 9 (2006) 260267.

[24] K.M. Dunn, M.T. Nelson, Potassium channels and neurovascular coupling, Circulation Journal, 74 (2010) 608-616.

[25] A.F. McCaslin, B.R. Chen, A.J. Radosevich, B. Cauli, E.M. Hillman, in vivo 3D Morphology of Astrocyte - Vasculature Interactions in the Somatosensory Cortex: Implications for Neurovascular Coupling, Journal of cerebral blood flow \& metabolism, 31 (2011) 795-806.

[26] K. Nizar, H. Uhlirova, P. Tian, P.A. Saisan, Q. Cheng, L. Reznichenko, K.L. Weldy, T.C. Steed, V.B. Sridhar, C.L. MacDonald, In vivo stimulus-induced vasodilation occurs 
without IP3 receptor activation and may precede astrocytic calcium increase, Journal of Neuroscience, 33 (2013) 8411-8422.

[27] W. Sun, E. McConnell, J.-F. Pare, Q. Xu, M. Chen, W. Peng, D. Lovatt, X. Han, Y. Smith, M. Nedergaard, Glutamate-dependent neuroglial calcium signaling differs between young and adult brain, Science, 339 (2013) 197-200.

[28] N. Takata, T. Nagai, K. Ozawa, Y. Oe, K. Mikoshiba, H. Hirase, Cerebral blood flow modulation by Basal forebrain or whisker stimulation can occur independently of large cytosolic Ca 2+ signaling in astrocytes, PLoS One, 8 (2013) e66525.

[29] H. Uhlirova, K. Kılıç, P. Tian, M. Thunemann, M. Desjardins, P.A. Saisan, S. Sakadžić, T.V. Ness, C. Mateo, Q. Cheng, Cell type specificity of neurovascular coupling in cerebral cortex, Elife, 5 (2016) e14315.

[30] T.A. Longden, F. Dabertrand, M. Koide, A.L. Gonzales, N.R. Tykocki, J.E. Brayden, D. Hill-Eubanks, M.T. Nelson, Capillary K+-sensing initiates retrograde hyperpolarization to increase local cerebral blood flow, Nature neuroscience, 20 (2017) 717.

[31] H. Hibino, A. Inanobe, K. Furutani, S. Murakami, I. Findlay, Y. Kurachi, Inwardly rectifying potassium channels: their structure, function, and physiological roles, Physiological reviews, 90 (2010) 291-366.

[32] A.N. Lopatin, E.N. Makhina, C.G. Nichols, Potassium channel block by cytoplasmic polyamines as the mechanism of intrinsic rectification, Nature, 372 (1994) 366.

[33] H. Matsuda, Open-state substructure of inwardly rectifying potassium channels revealed by magnesium block in guinea-pig heart cells, The Journal of Physiology, 397 (1988) 237-258.

[34] C. Nichols, A. Lopatin, Inward rectifier potassium channels, Annual review of physiology, 59 (1997) 171-191.

[35] K.M. Dunn, M.T. Nelson, Potassium channels and neurovascular coupling, Circulation journal: official journal of the Japanese Circulation Society, 74 (2010) 608.

[36] J.A. Filosa, A.D. Bonev, S.V. Straub, A.L. Meredith, M.K. Wilkerson, R.W. Aldrich, M.T. Nelson, Local potassium signaling couples neuronal activity to vasodilation in the brain, Nature neuroscience, 9 (2006) 1397.

[37] T.A. Longden, M.T. Nelson, Vascular inward rectifier K+ channels as external K+ sensors in the control of cerebral blood flow, Microcirculation, 22 (2015) 183-196.

[38] T.A. Longden, F. Dabertrand, D.C. Hill-Eubanks, S.E. Hammack, M.T. Nelson, Stress-induced glucocorticoid signaling remodels neurovascular coupling through impairment of cerebrovascular inwardly rectifying $\mathrm{K}+$ channel function, Proceedings of the National Academy of Sciences, 111 (2014) 7462-7467.

[39] J.M. Quayle, J.G. McCARRON, J.E. Brayden, M.T. Nelson, Inward rectifier K+ currents in smooth muscle cells from rat resistance-sized cerebral arteries, American Journal of Physiology-Cell Physiology, 265 (1993) C1363-C1370. 
[40] J.J. Zaritsky, D.M. Eckman, G.C. Wellman, M.T. Nelson, T.L. Schwarz, Targeted disruption of Kir2. 1 and Kir2. 2 genes reveals the essential role of the inwardly rectifying K+ current in K+-mediated vasodilation, Circulation Research, 87 (2000) 160166.

[41] P.D. Smith, S.E. Brett, K.D. Luykenaar, S.L. Sandow, S.P. Marrelli, E.J. Vigmond, D.G. Welsh, KIR channels function as electrical amplifiers in rat vascular smooth muscle, The Journal of physiology, 586 (2008) 1147-1160.

[42] H.M. Duvernoy, S. Delon, J. Vannson, Cortical blood vessels of the human brain, Brain research bulletin, 7 (1981) 519-579.

[43] T. Lovick, L. Brown, B. Key, Neurovascular relationships in hippocampal slices: physiological and anatomical studies of mechanisms underlying flow-metabolism coupling in intraparenchymal microvessels, Neuroscience, 92 (1999) 47-60.

[44] T.A. Longden, F. Dabertrand, A.L. Gonzales, M. Koide, M.T. Nelson, Potassium Sensing by Capillary KIR Channels Regulates Cerebral Blood Flow, Journal of General Physiology, ROCKEFELLER UNIV PRESS 950 THIRD AVE, 2ND FLR, NEW YORK, NY 10022 USA, 2015, pp. 10A-10A.

[45] O.F. Harraz, T.A. Longden, F. Dabertrand, D. Hill-Eubanks, M.T. Nelson, Endothelial GqPCR activity controls capillary electrical signaling and brain blood flow through PIP2 depletion, Proceedings of the National Academy of Sciences, 115 (2018) E3569-E3577.

[46] O.F. Harraz, T.A. Longden, D. Hill-Eubanks, M.T. Nelson, PIP2 depletion promotes TRPV4 channel activity in mouse brain capillary endothelial cells, Elife, 7 (2018) e38689.

[47] K.M. Dunn, M.T. Nelson, Potassium channels and neurovascular coupling, Circulation Journal, DOI (2010) 1003120644-1003120644.

[48] H. Girouard, A.D. Bonev, R.M. Hannah, A. Meredith, R.W. Aldrich, M.T. Nelson, Astrocytic endfoot $\mathrm{Ca} 2+$ and $\mathrm{BK}$ channels determine both arteriolar dilation and constriction, Proceedings of the National Academy of Sciences, 107 (2010) 3811-3816.

[49] T. Horiuchi, H.H. Dietrich, K. Hongo, R.G. Dacey Jr, Mechanism of extracellular $\mathrm{K}+$-induced local and conducted responses in cerebral penetrating arterioles, Stroke, 33 (2002) 2692-2699.

[50] J. McCarron, J. Quayle, W. Halpern, M. Nelson, Cromakalim and pinacidil dilate small mesenteric arteries but not small cerebral arteries, American Journal of PhysiologyHeart and Circulatory Physiology, 261 (1991) H287-H291.

[51] W.A. Coetzee, Y. Amarillo, J. Chiu, A. Chow, D. Lau, T. McCormack, H. Morena, M.S. Nadal, A. Ozaita, D. Pountney, Molecular diversity of K+ channels, Annals of the New York Academy of Sciences, 868 (1999) 233-255. 
[52] M. Koide, A. Moshkforoush, N.M. Tsoukias, D.C. Hill-Eubanks, G.C. Wellman, M.T. Nelson, F. Dabertrand, The yin and yang of KV channels in cerebral small vessel pathologies, Microcirculation, 25 (2018) e12436.

[53] K.C. Brennan, L. Beltrán-Parrazal, H.E. López-Valdés, J. Theriot, A.W. Toga, A.C. Charles, Distinct vascular conduction with cortical spreading depression, Journal of neurophysiology, 97 (2007) 4143-4151.

[54] D. Pietrobon, M.A. Moskowitz, Chaos and commotion in the wake of cortical spreading depression and spreading depolarizations, Nature Reviews Neuroscience, 15 (2014) 379.

[55] C. Ayata, M. Lauritzen, Spreading depression, spreading depolarizations, and the cerebral vasculature, Physiological reviews, 95 (2015) 953-993.

[56] M. Lauritzen, J.P. Dreier, M. Fabricius, J.A. Hartings, R. Graf, A.J. Strong, Clinical relevance of cortical spreading depression in neurological disorders: migraine, malignant stroke, subarachnoid and intracranial hemorrhage, and traumatic brain injury, Journal of Cerebral Blood Flow \& Metabolism, 31 (2011) 17-35.

[57] A.A. Leao, Spreading depression of activity in the cerebral cortex, Journal of neurophysiology, 7 (1944) 359-390.

[58] D. Torrente, R. Cabezas, M.F. Avila, L.M. García-Segura, G.E. Barreto, R.C.A. Guedes, Cortical spreading depression in traumatic brain injuries: is there a role for astrocytes?, Neuroscience letters, 565 (2014) 2-6.

[59] J.C. Chang, L.L. Shook, J. Biag, E.N. Nguyen, A.W. Toga, A.C. Charles, K.C. Brennan, Biphasic direct current shift, haemoglobin desaturation and neurovascular uncoupling in cortical spreading depression, Brain, 133 (2010) 996-1012.

[60] H. Piilgaard, M. Lauritzen, Persistent increase in oxygen consumption and impaired neurovascular coupling after spreading depression in rat neocortex, Journal of Cerebral Blood Flow \& Metabolism, 29 (2009) 1517-1527.

[61] M. Bennett, L. Farnell, W. Gibson, Origins of blood volume change due to glutamatergic synaptic activity at astrocytes abutting on arteriolar smooth muscle cells, Journal of theoretical biology, 250 (2008) 172-185.

[62] B.S. Chander, V.S. Chakravarthy, A computational model of neuro-glio-vascular loop interactions, PloS one, 7 (2012) e48802.

[63] E.J. Mathias, A. Kenny, M.J. Plank, T. David, Integrated models of neurovascular coupling and BOLD signals: Responses for varying neural activations, NeuroImage, 174 (2018) 69-86.

[64] I. Østby, L. Øyehaug, G.T. Einevoll, E.A. Nagelhus, E. Plahte, T. Zeuthen, C.M. Lloyd, O.P. Ottersen, S.W. Omholt, Astrocytic mechanisms explaining neural-activityinduced shrinkage of extraneuronal space, PLoS computational biology, 5 (2009) e1000272. 
[65] A. Witthoft, G.E. Karniadakis, A bidirectional model for communication in the neurovascular unit, Journal of Theoretical biology, 311 (2012) 80-93.

[66] E. De Schutter, P. Smolen, Calcium dynamics in large neuronal models, Methods in neuronal modeling: From ions to networks, 2 (1998).

[67] D.M. Santucci, S. Raghavachari, The effects of NR2 subunit-dependent NMDA receptor kinetics on synaptic transmission and CaMKII activation, PLoS computational biology, 4 (2008) e1000208.

[68] A. Witthoft, J.A. Filosa, G.E. Karniadakis, Potassium buffering in the neurovascular unit: models and sensitivity analysis, Biophysical journal, 105 (2013) 2046-2054.

[69] Y. Behzadi, T.T. Liu, An arteriolar compliance model of the cerebral blood flow response to neural stimulus, Neuroimage, 25 (2005) 1100-1111.

[70] R.B. Buxton, K. Uludağ, D.J. Dubowitz, T.T. Liu, Modeling the hemodynamic response to brain activation, Neuroimage, 23 (2004) S220-S233.

[71] K.J. Friston, A. Mechelli, R. Turner, C.J. Price, Nonlinear responses in fMRI: the Balloon model, Volterra kernels, and other hemodynamics, NeuroImage, 12 (2000) 466477.

[72] T.J. Huppert, M.S. Allen, H. Benav, P.B. Jones, D.A. Boas, A multicompartment vascular model for inferring baseline and functional changes in cerebral oxygen metabolism and arterial dilation, Journal of cerebral blood flow \& metabolism, 27 (2007) 1262-1279.

[73] J.H. Kim, D. Ress, Arterial impulse model for the BOLD response to brief neural activation, NeuroImage, 124 (2016) 394-408.

[74] R. Mesquita, T. Huppert, D. Boas, Exploring neuro-vascular and neuro-metabolic coupling in rat somatosensory cortex, Physics in Medicine \& Biology, 54 (2008) 175.

[75] K.L. Miller, W.M. Luh, T.T. Liu, A. Martinez, T. Obata, E.C. Wong, L.R. Frank, R.B. Buxton, Nonlinear temporal dynamics of the cerebral blood flow response, Human brain mapping, 13 (2001) 1-12.

[76] J.J. Riera, J.C. Jimenez, X. Wan, R. Kawashima, T. Ozaki, Nonlinear local electrovascular coupling. II: From data to neuronal masses, Human brain mapping, 28 (2007) 335-354.

[77] J.J. Riera, X. Wan, J.C. Jimenez, R. Kawashima, Nonlinear local electrovascular coupling. I: A theoretical model, Human brain mapping, 27 (2006) 896-914.

[78] P.A. Valdes-Hernandez, B. Bernal, A. Moshkforoush, C. Dunoyer, H.M. Khoo, J. Bosch-Bayard, N. von Ellenrieder, J. Gotman, J.J. Riera, Identification of negative BOLD responses using windkessel models, bioRxiv, DOI (2018) 392290.

[79] Y. Zheng, Y. Pan, S. Harris, S. Billings, D. Coca, J. Berwick, M. Jones, A. Kennerley, D. Johnston, C. Martin, A dynamic model of neurovascular coupling: 
implications for blood vessel dilation and constriction, Neuroimage, 52 (2010) 11351147.

[80] C.S. Roy, C.S. Sherrington, On the regulation of the blood-supply of the brain, The Journal of physiology, 11 (1890) 85-158.

[81] M.E. Raichle, M.A. Mintun, Brain work and brain imaging, Annu. Rev. Neurosci., 29 (2006) 449-476.

[82] C. Iadecola, M. Nedergaard, Glial regulation of the cerebral microvasculature, Nature neuroscience, 10 (2007) 1369.

[83] M. Zonta, M.C. Angulo, S. Gobbo, B. Rosengarten, K.-A. Hossmann, T. Pozzan, G. Carmignoto, Neuron-to-astrocyte signaling is central to the dynamic control of brain microcirculation, Nature neuroscience, 6 (2003) 43.

[84] M. Zonta, M.C. Angulo, S. Gobbo, B. Rosengarten, K.A. Hossmann, T. Pozzan, G. Carmignoto, Neuron-to-astrocyte signaling is central to the dynamic control of brain microcirculation, Nature Neuroscience, 6 (2003) 43-50.

[85] T.A. Longden, F. Dabertrand, M. Koide, A.L. Gonzales, N.R. Tykocki, J.E. Brayden, D. Hill-Eubanks, M.T. Nelson, Capillary K+-sensing initiates retrograde hyperpolarization to increase local cerebral blood flow, Nature Neuroscience, DOI (2017).

[86] C.N. Hall, C. Reynell, B. Gesslein, N.B. Hamilton, A. Mishra, B.A. Sutherland, F.M. O'farrell, A.M. Buchan, M. Lauritzen, D. Attwell, Capillary pericytes regulate cerebral blood flow in health and disease, Nature, 508 (2014) 55.

[87] B. Nilius, G. Droogmans, Ion channels and their functional role in vascular endothelium, Physiological reviews, 81 (2001) 1415-1459.

[88] M.R. Silver, M.S. Shapiro, T.E. DeCoursey, Effects of external Rb+ on inward rectifier $\mathrm{K}+$ channels of bovine pulmonary artery endothelial cells, The Journal of general physiology, 103 (1994) 519-548.

[89] H.S. Silva, A. Kapela, N.M. Tsoukias, A mathematical model of plasma membrane electrophysiology and calcium dynamics in vascular endothelial cells, American Journal of Physiology-Cell Physiology, DOI (2007).

[90] Y. Yang, F. Chen, T. Karasawa, K.-T. Ma, B.-C. Guan, X.-R. Shi, H. Li, P.S.

Steyger, A.L. Nuttall, Z.-G. Jiang, Diverse Kir expression contributes to distinct bimodal distribution of resting potentials and vasotone responses of arterioles, PloS one, 10 (2015) e0125266.

[91] D. Colquhoun, A. Hawkes, On the stochastic properties of single ion channels, Proceedings of the Royal Society of London. Series B. Biological Sciences, 211 (1981) 205-235.

[92] J. Parikh, A. Kapela, N.M. Tsoukias, Stochastic model of endothelial TRPV4 calcium sparklets: effect of bursting and cooperativity on EDH, Biophysical journal, 108 (2015) 1566-1576. 
[93] S.K. Sonkusare, A.D. Bonev, J. Ledoux, W. Liedtke, M.I. Kotlikoff, T.J. Heppner, D.C. Hill-Eubanks, M.T. Nelson, Elementary Ca2+ signals through endothelial TRPV4 channels regulate vascular function, Science, 336 (2012) 597-601.

[94] A. Kapela, E.J. Behringer, S.S. Segal, N.M. Tsoukias, Biophysical properties of microvascular endothelium: Requirements for initiating and conducting electrical signals, Microcirculation, 25 (2018) e12429.

[95] Y. Yamamoto, K. Imaeda, H. Suzuki, Endothelium-dependent hyperpolarization and intercellular electrical coupling in guinea-pig mesenteric arterioles, The Journal of Physiology, 514 (1999) 505-513.

[96] P. Blinder, P.S. Tsai, J.P. Kaufhold, P.M. Knutsen, H. Suhl, D. Kleinfeld, The cortical angiome: an interconnected vascular network with noncolumnar patterns of blood flow, Nature neuroscience, 16 (2013) 889.

[97] A. Kapela, S. Nagaraja, N.M. Tsoukias, A mathematical model of vasoreactivity in rat mesenteric arterioles: II Conducted vasoreactivity, American Journal of PhysiologyHeart and Circulatory Physiology, DOI (2009).

[98] T. Voets, G. Droogmans, B. Nilius, Membrane currents and the resting membrane potential in cultured bovine pulmonary artery endothelial cells, The Journal of physiology, 497 (1996) 95-107.

[99] T.R. Elam, J.B. Lansman, The role of Mg2+ in the inactivation of inwardly rectifying $\mathrm{K}+$ channels in aortic endothelial cells, The Journal of general physiology, 105 (1995) 463-484.

[100] D. Postnov, A. Neganova, O. Sosnovtseva, N.-H. Holstein-Rathlou, J.B. Jacobsen, Conducted vasoreactivity: the dynamical point of view, Bulletin of mathematical biology, 77 (2015) 230-249.

[101] J. McCarron, W. Halpern, Impaired potassium-induced dilation in hypertensive rat cerebral arteries does not reflect altered $\mathrm{Na}+, \mathrm{K}(+)$-ATPase dilation, Circulation Research, 67 (1990) 1035-1039.

[102] J.G. McCarron, W. Halpern, Potassium dilates rat cerebral arteries by two independent mechanisms, American Journal of Physiology-Heart and Circulatory Physiology, 259 (1990) H902-H908.

[103] M.C. Jantzi, S.E. Brett, W.F. Jackson, R.L. Corteling, E.J. Vigmond, D.G. Welsh, Inward rectifying potassium channels facilitate cell-to-cell communication in hamster retractor muscle feed arteries, American Journal of Physiology-Heart and Circulatory Physiology, DOI (2006).

[104] E.J. Behringer, S.S. Segal, Tuning electrical conduction along endothelial tubes of resistance arteries through Ca2+-activated K+ channels, Circulation research, 110 (2012) $1311-1321$. 
[105] S.S. Segal, B.R. Duling, Conduction of vasomotor responses in arterioles: a role for cell-to-cell coupling?, American Journal of Physiology-Heart and Circulatory

Physiology, 256 (1989) H838-H845.

[106] A.N. Lopatin, E.N. Makhina, C.G. Nichols, Potassium channel block by cytoplasmic polyamines as the mechanism of intrinsic rectification, Nature, 372 (1994) 366-369.

[107] J.M. Quayle, M.T. Nelson, N.B. Standen, ATP-sensitive and inwardly rectifying potassium channels in smooth muscle, Physiol Rev, 77 (1997) 1165-1232.

[108] T.A. Longden, M.T. Nelson, Vascular Inward Rectifier K Channels as External K Sensors in the Control of Cerebral Blood Flow, Microcirculation, DOI 10.1111/micc.12190(2015).

[109] G. Mehrke, U. Pohl, J. Daut, Effects of vasoactive agonists on the membrane potential of cultured bovine aortic and guinea-pig coronary endothelium, J Physiol, 439 (1991) 277-299.

[110] T. Voets, G. Droogmans, B. Nilius, Membrane currents and the resting membrane potential in cultured bovine pulmonary artery endothelial cells, J Physiol, 497 ( Pt 1) (1996) 95-107.

[111] B. Nilius, G. Droogmans, Ion channels and their functional role in vascular endothelium, Physiological reviews, 81 (2001) 1415-1459.

[112] H.S. Silva, A. Kapela, N.M. Tsoukias, A mathematical model of plasma membrane electrophysiology and calcium dynamics in vascular endothelial cells, Am J Physiol Cell Physiol, 293 (2007) C277-293.

[113] J.G. McCarron, W. Halpern, Impaired potassium-induced dilation in hypertensive rat cerebral arteries does not reflect altered $\mathrm{Na}+\mathrm{K}(+)$-ATPase dilation, Circ Res, 67 (1990) 1035-1039.

[114] A.L. Hodgkin, A.F. Huxley, A quantitative description of membrane current and its application to conduction and excitation in nerve, The Journal of physiology, 117 (1952) 500-544.

[115] G. Blanco, R.W. Mercer, Isozymes of the Na-K-ATPase: heterogeneity in structure, diversity in function, American Journal of Physiology-Renal Physiology, 275 (1998) F633-F650.

[116] L. Pantoni, Cerebral small vessel disease: from pathogenesis and clinical characteristics to therapeutic challenges, The Lancet Neurology, 9 (2010) 689-701.

[117] J.B. Bederson, E.S. Connolly Jr, H.H. Batjer, R.G. Dacey, J.E. Dion, M.N. Diringer, J.E. Duldner Jr, R.E. Harbaugh, A.B. Patel, R.H. Rosenwasser, Guidelines for the management of aneurysmal subarachnoid hemorrhage: a statement for healthcare professionals from a special writing group of the Stroke Council, American Heart Association, Stroke, 40 (2009) 994-1025. 
[118] C. Capone, F. Dabertrand, C. Baron-Menguy, A. Chalaris, L. Ghezali, V. Domenga-Denier, S. Schmidt, C. Huneau, S. Rose-John, M.T. Nelson, Mechanistic insights into a TIMP3-sensitive pathway constitutively engaged in the regulation of cerebral hemodynamics, Elife, 5 (2016) e17536.

[119] H. Chabriat, A. Joutel, M. Dichgans, E. Tournier-Lasserve, M.-G. Bousser, Cadasil, The Lancet Neurology, 8 (2009) 643-653.

[120] F. Dabertrand, C. Krøigaard, A.D. Bonev, E. Cognat, T. Dalsgaard, V. DomengaDenier, D.C. Hill-Eubanks, J.E. Brayden, A. Joutel, M.T. Nelson, Potassium channelopathy-like defect underlies early-stage cerebrovascular dysfunction in a genetic model of small vessel disease, Proceedings of the National Academy of Sciences, 112 (2015) E796-E805.

[121] M. Ishiguro, A.D. Morielli, K. Zvarova, B.I. Tranmer, P.L. Penar, G.C. Wellman, Oxyhemoglobin-induced suppression of voltage-dependent $\mathrm{K}+$ channels in cerebral arteries by enhanced tyrosine kinase activity, Circulation research, 99 (2006) 1252-1260.

[122] A. Joutel, I. Haddad, J. Ratelade, M.T. Nelson, Perturbations of the cerebrovascular matrisome: A convergent mechanism in small vessel disease of the brain?, Journal of Cerebral Blood Flow \& Metabolism, 36 (2016) 143-157.

[123] M. Koide, P.L. Penar, B.I. Tranmer, G.C. Wellman, Heparin-binding EGF-like growth factor mediates oxyhemoglobin-induced suppression of voltage-dependent potassium channels in rabbit cerebral artery myocytes, American Journal of PhysiologyHeart and Circulatory Physiology, 293 (2007) H1750-H1759.

[124] G.A. Gutman, K.G. Chandy, S. Grissmer, M. Lazdunski, D. Mckinnon, L.A. Pardo, G.A. Robertson, B. Rudy, M.C. Sanguinetti, W. Stühmer, International Union of Pharmacology. LIII. Nomenclature and molecular relationships of voltage-gated potassium channels, Pharmacological reviews, 57 (2005) 473-508.

[125] M. Koide, G.C. Wellman, SAH-Induced Suppression of Voltage-Gated K+(K V) Channel Currents in Parenchymal Arteriolar Myocytes Involves Activation of the HBEGF/EGFR Pathway, Cerebral Vasospasm: Neurovascular Events After Subarachnoid Hemorrhage, Springer2013, pp. 179-184.

[126] S.V. Straub, H. Girouard, P.E. Doetsch, R.M. Hannah, M.K. Wilkerson, M.T. Nelson, Regulation of intracerebral arteriolar tone by Kv channels: effects of glucose and PKC, American Journal of Physiology-Cell Physiology, 297 (2009) C788-C796.

[127] M.T. Nelson, J.M. Quayle, Physiological roles and properties of potassium channels in arterial smooth muscle, American Journal of Physiology-Cell Physiology, 268 (1995) C799-C822.

[128] S.V. Straub, H. Girouard, P.E. Doetsch, R.M. Hannah, M.K. Wilkerson, M.T. Nelson, Regulation of intracerebral arteriolar tone by K v channels: effects of glucose and PKC, American Journal of Physiology-Cell Physiology, 297 (2009) C788-C796. 
[129] S. Albarwani, L.T. Nemetz, J.A. Madden, A.A. Tobin, S.K. England, P.F. Pratt, N.J. Rusch, Voltage-gated K+ channels in rat small cerebral arteries: molecular identity of the functional channels, The Journal of physiology, 551 (2003) 751-763.

[130] A. Cheong, A. Dedman, D. Beech, Expression and function of native potassium channel $(\mathrm{Kv} \alpha 1)$ subunits in terminal arterioles of rabbit, The Journal of physiology, 534 (2001) 691-700.

[131] A. Cheong, A. Dedman, S. Xu, D. Beech, K V $\alpha 1$ channels in murine arterioles: differential cellular expression and regulation of diameter, American Journal of Physiology-Heart and Circulatory Physiology, 281 (2001) H1057-H1065.

[132] G.C. Amberg, L.F. Santana, Kv2 channels oppose myogenic constriction of rat cerebral arteries, American Journal of Physiology-Cell Physiology, 291 (2006) C348C356.

[133] G.C. Amberg, C.F. Rossow, M.F. Navedo, L.F. Santana, NFATc3 regulates Kv2. 1 expression in arterial smooth muscle, Journal of Biological Chemistry, 279 (2004) 47326-47334.

[134] X.Z. Zhong, K.S. Abd-Elrahman, C.H. Liao, A.F. El-Yazbi, E.J. Walsh, M.P. Walsh, W.C. Cole, Stromatoxin-sensitive, heteromultimeric Kv2. 1/Kv9. 3 channels contribute to myogenic control of cerebral arterial diameter, The Journal of physiology, 588 (2010) 4519-4537.

[135] X.Z. Zhong, M.I. Harhun, S.P. Olesen, S. Ohya, J.D. Moffatt, W.C. Cole, I.A. Greenwood, Participation of KCNQ (Kv7) potassium channels in myogenic control of cerebral arterial diameter, The Journal of physiology, 588 (2010) 3277-3293.

[136] T.A. Jepps, S. Olesen, I. Greenwood, One man's side effect is another man's therapeutic opportunity: targeting Kv7 channels in smooth muscle disorders, British journal of pharmacology, 168 (2013) 19-27.

[137] M.J. Davis, M.A. Hill, Signaling mechanisms underlying the vascular myogenic response, Physiological reviews, 79 (1999) 387-423.

[138] W.F. Jackson, Potassium channels in the peripheral microcirculation, Microcirculation, 12 (2005) 113-127.

[139] N.R. Tykocki, E.M. Boerman, W.F. Jackson, Smooth muscle ion channels and regulation of vascular tone in resistance arteries and arterioles, Comprehensive Physiology, 7 (2011) 485-581.

[140] F. Dabertrand, M.T. Nelson, J.E. Brayden, Acidosis dilates brain parenchymal arterioles by conversion of calcium waves to sparks to activate BK channels, Circulation research, 110 (2012) 285-294.

[141] R.M. Hannah, K.M. Dunn, A.D. Bonev, M.T. Nelson, Endothelial SKCa and IKCa channels regulate brain parenchymal arteriolar diameter and cortical cerebral blood flow, Journal of Cerebral Blood Flow \& Metabolism, 31 (2011) 1175-1186. 
[142] G.K. Povlsen, T.A. Longden, A.D. Bonev, D.C. Hill-Eubanks, M.T. Nelson, Uncoupling of neurovascular communication after transient global cerebral ischemia is caused by impaired parenchymal smooth muscle Kir channel function, Journal of Cerebral Blood Flow \& Metabolism, 36 (2016) 1195-1201.

[143] W.M. Bayliss, On the local reactions of the arterial wall to changes of internal pressure, The Journal of physiology, 28 (1902) 220-231.

[144] M.A. Nystoriak, K.P. O'Connor, S.K. Sonkusare, J.E. Brayden, M.T. Nelson, G.C. Wellman, Fundamental increase in pressure-dependent constriction of brain parenchymal arterioles from subarachnoid hemorrhage model rats due to membrane depolarization, American Journal of Physiology-Heart and Circulatory Physiology, 300 (2010) H803H812.

[145] A. Kapela, A. Bezerianos, N.M. Tsoukias, A mathematical model of Ca2+ dynamics in rat mesenteric smooth muscle cell: agonist and NO stimulation, Journal of theoretical biology, 253 (2008) 238-260.

[146] M.J. Cipolla, J. Sweet, S.-L. Chan, M.J. Tavares, N. Gokina, J.E. Brayden, Increased pressure-induced tone in rat parenchymal arterioles vs. middle cerebral arteries: role of ion channels and calcium sensitivity, Journal of applied physiology, 117 (2014) 53-59.

[147] A. Shah, I. Cohen, N. Datyner, Background K+ current in isolated canine cardiac Purkinje myocytes, Biophysical journal, 52 (1987) 519-525.

[148] M. Balbi, M. Koide, G.C. Wellman, N. Plesnila, Inversion of neurovascular coupling after subarachnoid hemorrhage in vivo, Journal of Cerebral Blood Flow \& Metabolism, 37 (2017) 3625-3634.

[149] M. Koide, A.D. Bonev, M.T. Nelson, G.C. Wellman, Inversion of neurovascular coupling by subarachnoid blood depends on large-conductance Ca2+-activated $\mathrm{K}+(\mathrm{BK})$ channels, Proceedings of the National Academy of Sciences, 109 (2012) E1387-E1395.

[150] A.C. Pappas, M. Koide, G.C. Wellman, Purinergic signaling triggers endfoot highamplitude $\mathrm{Ca} 2+$ signals and causes inversion of neurovascular coupling after subarachnoid hemorrhage, Journal of Cerebral Blood Flow \& Metabolism, 36 (2016) 1901-1912.

[151] A. Moshkforoush, P.A. Valdes-Hernandez, D.E. Rivera-Espada, Y. Mori, J. Riera, waveCSD: A method for estimating transmembrane currents originated from propagating neuronal activity in the neocortex: Application to study cortical spreading depression, Journal of neuroscience methods, 307 (2018) 106-124.

[152] S. Łęski, K.H. Pettersen, B. Tunstall, G.T. Einevoll, J. Gigg, D.K. Wójcik, Inverse current source density method in two dimensions: inferring neural activation from multielectrode recordings, Neuroinformatics, 9 (2011) 401-425.

[153] J. Potworowski, W. Jakuczun, S. Łęski, D. Wójcik, Kernel current source density method, Neural computation, 24 (2012) 541-575. 
[154] S. Łęski, D.K. Wójcik, J. Tereszczuk, D.A. Świejkowski, E. Kublik, A. Wróbel, Inverse current-source density method in 3D: reconstruction fidelity, boundary effects, and influence of distant sources, Neuroinformatics, 5 (2007) 207-222.

[155] J.J. Riera, T. Goto, R. Kawashima, A methodology for fast assessments to the electrical activity of barrel fields in vivo: from population inputs to single unit outputs, Frontiers in neural circuits, 8 (2014).

[156] J.J. Riera, T. Ogawa, T. Goto, A. Sumiyoshi, H. Nonaka, A. Evans, H. Miyakawa, R. Kawashima, Pitfalls in the dipolar model for the neocortical EEG sources, Journal of neurophysiology, 108 (2012) 956-975.

[157] J. Freeman, C. Nicholson, Experimental optimization of current source-density technique for anuran cerebellum, Journal of Neurophysiology, 38 (1975) 369-382.

[158] U. Mitzdorf, Current source-density method and application in cat cerebral cortex: investigation of evoked potentials and EEG phenomena, DOI (1985).

[159] C. Nicholson, J.A. Freeman, Theory of current source-density analysis and determination of conductivity tensor for anuran cerebellum, Journal of neurophysiology, 38 (1975) 356-368.

[160] C. Nicholson, R. Llinas, Real time current source-density analysis using multielectrode array in cat cerebellum, Brain research, 100 (1975) 418-424.

[161] K.H. Pettersen, A. Devor, I. Ulbert, A.M. Dale, G.T. Einevoll, Current-source density estimation based on inversion of electrostatic forward solution: effects of finite extent of neuronal activity and conductivity discontinuities, Journal of neuroscience methods, 154 (2006) 116-133.

[162] G.T. Einevoll, C. Kayser, N.K. Logothetis, S. Panzeri, Modelling and analysis of local field potentials for studying the function of cortical circuits, Nature Reviews Neuroscience, 14 (2013) 770-785.

[163] C.E. Schroeder, A.D. Mehta, S.J. Givre, A spatiotemporal profile of visual system activation revealed by current source density analysis in the awake macaque, Cerebral cortex (New York, NY: 1991), 8 (1998) 575-592.

[164] V.A. Aroniadou, A. Keller, The patterns and synaptic properties of horizontal intracortical connections in the rat motor cortex, Journal of neurophysiology, 70 (1993) 1553-1569.

[165] D.S. Barth, C. Baumgartner, S. Di, Laminar interactions in rat motor cortex during cyclical excitability changes of the penicillin focus, Brain research, 508 (1990) 105-117.

[166] D.C. Godlove, A. Maier, G.F. Woodman, J.D. Schall, Microcircuitry of agranular frontal cortex: testing the generality of the canonical cortical microcircuit, Journal of Neuroscience, 34 (2014) 5355-5369.

[167] F. Lopes da Silva, W. Storm van Leeuwen, The cortical alpha rhythm in dog: the depth and surface profile of phase, Raven Press New York1978. 
[168] P.L. Nunez, Wavelike properties of the alpha rhythm, IEEE Transactions on Biomedical Engineering, DOI (1974) 473-482.

[169] P.L. Nunez, B.A. Cutillo, Neocortical dynamics and human EEG rhythms, Oxford University Press, USA1995.

[170] G.R. Burkitt, R.B. Silberstein, P.J. Cadusch, A.W. Wood, Steady-state visual evoked potentials and travelling waves, Clinical Neurophysiology, 111 (2000) 246-258.

[171] J.R. Hughes, A. Ikram, J.J. Fino, Characteristics of travelling waves under various conditions, Clinical Electroencephalography, 26 (1995) 7-22.

[172] J.R. Hughes, A. Kuruvilla, J.J. Fino, Topographic analysis of visual evoked potentials from flash and pattern reversal stimuli: evidence for "travelling waves", Brain topography, 4 (1992) 215-228.

[173] M. Massimini, R. Huber, F. Ferrarelli, S. Hill, G. Tononi, The sleep slow oscillation as a traveling wave, Journal of Neuroscience, 24 (2004) 6862-6870.

[174] J.-Y. Wu, X. Huang, C. Zhang, Propagating waves of activity in the neocortex: what they are, what they do, The Neuroscientist, 14 (2008) 487-502.

[175] M. Lauritzen, Pathophysiology of the migraine aura: the spreading depression theory, Brain, 117 (1994) 199-210.

[176] A.A. Leo, Spreading depression of activity in the cerebral cortex, Journal of neurophysiology, 7 (1944) 359-390.

[177] H.R. Wilson, R. Blake, S.-H. Lee, Dynamics of travelling waves in visual perception, Nature, 412 (2001) 907-910.

[178] T.P. Zanos, P.J. Mineault, K.T. Nasiotis, D. Guitton, C.C. Pack, A sensorimotor role for traveling waves in primate visual cortex, Neuron, 85 (2015) 615-627.

[179] L. Muller, F. Chavane, J. Reynolds, T.J. Sejnowski, Cortical travelling waves: mechanisms and computational principles, Nature Reviews Neuroscience, DOI (2018).

[180] T. Goto, R. Hatanaka, T. Ogawa, A. Sumiyoshi, J. Riera, R. Kawashima, An evaluation of the conductivity profile in the somatosensory barrel cortex of Wistar rats, Journal of neurophysiology, 104 (2010) 3388-3412.

[181] A.N. Tikhonov, V.I.A.k. Arsenin, F. John, Solutions of ill-posed problems, Winston Washington, DC1977.

[182] G. Wahba, Spline models for observational data, Siam1990.

[183] A. Gramfort, T. Papadopoulo, E. Olivi, M. Clerc, OpenMEEG: opensource software for quasistatic bioelectromagnetics, Biomedical engineering online, 9 (2010) 45 .

[184] Y. Song, R.A. Torres, S. Garcia, Y. Frometa, J. Bae, A. Deshmukh, W.-C. Lin, Y. Zheng, J.J. Riera, Dysfunction of Neurovascular/Metabolic Coupling in Chronic Focal Epilepsy, IEEE Transactions on Biomedical Engineering, 63 (2016) 97-110. 
[185] K. Rohr, H.S. Stiehl, R. Sprengel, T.M. Buzug, J. Weese, M. Kuhn, Landmarkbased elastic registration using approximating thin-plate splines, IEEE Transactions on medical imaging, 20 (2001) 526-534.

[186] G.E. Box, D.R. Cox, An analysis of transformations, Journal of the Royal Statistical Society. Series B (Methodological), DOI (1964) 211-252.

[187] A. Gorji, Spreading depression: a review of the clinical relevance, Brain Research Reviews, 38 (2001) 33-60.

[188] B. Grafstein, Mechanism of spreading cortical depression, Journal of neurophysiology, 19 (1956) 154-171.

[189] P. Berens, G.A. Keliris, A.S. Ecker, N.K. Logothetis, A.S. Tolias, Feature selectivity of the gamma-band of the local field potential in primate primary visual cortex, Frontiers in neuroscience, 2 (2008) 199.

[190] Y. Kajikawa, C.E. Schroeder, How local is the local field potential?, Neuron, 72 (2011) 847-858.

[191] G. Kreiman, C.P. Hung, A. Kraskov, R.Q. Quiroga, T. Poggio, J.J. DiCarlo, Object selectivity of local field potentials and spikes in the macaque inferior temporal cortex, Neuron, 49 (2006) 433-445.

[192] I. Nauhaus, L. Busse, M. Carandini, D.L. Ringach, Stimulus contrast modulates functional connectivity in visual cortex, Nature neuroscience, 12 (2009) 70-76.

[193] C. Wang, I. Ulbert, D.L. Schomer, K. Marinkovic, E. Halgren, Responses of human anterior cingulate cortex microdomains to error detection, conflict monitoring, stimulusresponse mapping, familiarity, and orienting, Journal of Neuroscience, 25 (2005) 604613.

[194] H. Lindén, T. Tetzlaff, T.C. Potjans, K.H. Pettersen, S. Grün, M. Diesmann, G.T. Einevoll, Modeling the spatial reach of the LFP, Neuron, 72 (2011) 859-872.

[195] S. Katzner, I. Nauhaus, A. Benucci, V. Bonin, D.L. Ringach, M. Carandini, Local origin of field potentials in visual cortex, Neuron, 61 (2009) 35-41.

[196] D. Xing, C.-I. Yeh, R.M. Shapley, Spatial spread of the local field potential and its laminar variation in visual cortex, Journal of neuroscience, 29 (2009) 11540-11549.

[197] X. Huang, W. Xu, J. Liang, K. Takagaki, X. Gao, J.-y. Wu, Spiral wave dynamics in neocortex, Neuron, 68 (2010) 978-990.

[198] W. Wadman, A. Juta, W. Kamphuis, G. Somjen, Current source density of sustained potential shifts associated with electrographic seizures and with spreading depression in rat hippocampus, Brain research, 570 (1992) 85-91.

[199] J. Makarova, M. Gómez-Galán, O. Herreras, Variations in tissue resistivity and in the extension of activated neuron domains shape the voltage signal during spreading depression in the CA1 in vivo, European Journal of Neuroscience, 27 (2008) 444-456. 
[200] R. Khazipov, A. Nasretdinov, N. Lotfullina, G. Burkhanova, K. Chernova, A. Zakharov, Direct Current Coupled Recordings of Cortical Spreading Depression Using Silicone Probes, Frontiers in cellular neuroscience, 11 (2017) 408.

[201] T.A. Basarsky, S.N. Duffy, R.D. Andrew, B.A. MacVicar, Imaging spreading depression and associated intracellular calcium waves in brain slices, Journal of Neuroscience, 18 (1998) 7189-7199.

[202] F. Richter, A. Lehmenkühler, Spreading depression can be restricted to distinct depths of the rat cerebral cortex, Neuroscience letters, 152 (1993) 65-68.

[203] A. Leao, R. Morison, Propagation of spreading cortical depression, The Journal of Nervous and Mental Disease, 102 (1945) 512.

[204] A.A. Leão, The slow voltage variation of cortical spreading depression of activity, Electroencephalography and clinical neurophysiology, 3 (1951) 315-321.

[205] P.E. Kunkler, R.P. Kraig, Calcium waves precede electrophysiological changes of spreading depression in hippocampal organ cultures, Journal of Neuroscience, 18 (1998) 3416-3425.

[206] O. Peters, C.G. Schipke, Y. Hashimoto, H. Kettenmann, Different mechanisms promote astrocyte $\mathrm{Ca} 2+$ waves and spreading depression in the mouse neocortex, Journal of Neuroscience, 23 (2003) 9888-9896.

[207] A. Cabo, J.J. Riera, How the active and diffusional nature of brain tissues can generate monopole signals at micrometer sized measures, arXiv preprint arXiv:1410.0274, DOI (2014).

[208] A. Destexhe, C. Bedard, Do neurons generate monopolar current sources?, Journal of neurophysiology, 108 (2012) 953-955.

[209] S.L. Gratiy, K.H. Pettersen, G.T. Einevoll, A.M. Dale, Pitfalls in the interpretation of multielectrode data: on the infeasibility of the neuronal current-source monopoles, Journal of neurophysiology, 109 (2013) 1681-1682.

[210] J. Riera, A. Cabo, Reply to Gratiy et al, Journal of neurophysiology, 109 (2013) 1684-1685.

[211] G. Halnes, T. Mäki-Marttunen, D. Keller, K.H. Pettersen, O.A. Andreassen, G.T. Einevoll, Effect of ionic diffusion on extracellular potentials in neural tissue, PLoS computational biology, 12 (2016) e1005193.

[212] N. Qian, T. Sejnowski, An electro-diffusion model for computing membrane potentials and ionic concentrations in branching dendrites, spines and axons, Biological Cybernetics, 62 (1989) 1-15.

[213] D. Feuerstein, A. Manning, P. Hashemi, R. Bhatia, M. Fabricius, C. Tolias, C. Pahl, M. Ervine, A.J. Strong, M.G. Boutelle, Dynamic metabolic response to multiple spreading depolarizations in patients with acute brain injury: an online microdialysis study, Journal of Cerebral Blood Flow \& Metabolism, 30 (2010) 1343-1355. 
[214] D.R. Kramer, T. Fujii, I. Ohiorhenuan, C.Y. Liu, Cortical spreading depolarization: pathophysiology, implications, and future directions, Journal of Clinical Neuroscience, 24 (2016) 22-27.

[215] T.P. Obrenovitch, J. Urenjak, M. Wang, Nitric oxide formation during cortical spreading depression is critical for rapid subsequent recovery of ionic homeostasis, Journal of Cerebral Blood Flow \& Metabolism, 22 (2002) 680-688.

[216] J. Sword, D. Croom, P.L. Wang, R.J. Thompson, S.A. Kirov, Neuronal pannexin-1 channels are not molecular routes of water influx during spreading depolarizationinduced dendritic beading, Journal of Cerebral Blood Flow \& Metabolism, 37 (2017) 1626-1633.

[217] Y. Mori, A multidomain model for ionic electrodiffusion and osmosis with an application to cortical spreading depression, Physica D: Nonlinear Phenomena, 308 (2015) 94-108.

[218] W.H. Press, S.A. Teukolsky, W.T. Vetterling, B.P. Flannery, Numerical recipes in C, Cambridge university press Cambridge1996.

[219] H.C. Tuckwell, R.M. Miura, A mathematical model for spreading cortical depression, Biophysical Journal, 23 (1978) 257-276.

[220] B.E. Shapiro, Osmotic forces and gap junctions in spreading depression: a computational model, Journal of computational neuroscience, 10 (2001) 99-120.

[221] M.R. Bennett, L. Farnell, W.G. Gibson, A quantitative model of cortical spreading depression due to purinergic and gap-junction transmission in astrocyte networks, Biophysical journal, 95 (2008) 5648-5660.

[222] H. Huang, R.M. Miura, W. Yao, A simplified neuronal model for the instigation and propagation of cortical spreading depression, Advances in Applied Mathematics and Mechanics, 3 (2011) 759-773.

[223] H. Kager, W. Wadman, G. Somjen, Conditions for the triggering of spreading depression studied with computer simulations, Journal of neurophysiology, 88 (2002) 2700-2712.

[224] A. Kenny, M.J. Plank, T. David, Macro scale modelling of cortical spreading depression and the role of astrocytic gap junctions, Journal of theoretical biology, 458 (2018) 78-91.

[225] R.M. Miura, H. Huang, J.J. Wylie, Mathematical approaches to modeling of cortical spreading depression, Chaos: An Interdisciplinary Journal of Nonlinear Science, 23 (2013) 046103.

[226] B.-J. Zandt, B. ten Haken, M.J. van Putten, M.A. Dahlem, How does spreading depression spread? Physiology and modeling, Reviews in the Neurosciences, 26 (2015) 183-198. 
[227] J.C. Chang, K.C. Brennan, D. He, H. Huang, R.M. Miura, P.L. Wilson, J.J. Wylie, A mathematical model of the metabolic and perfusion effects on cortical spreading depression, PLoS One, 8 (2013) e70469.

[228] W. Walz, Role of astrocytes in the clearance of excess extracellular potassium, Neurochemistry international, 36 (2000) 291-300.

[229] P. Kofuji, E. Newman, Potassium buffering in the central nervous system, Neuroscience, 129 (2004) 1043-1054.

[230] C. Nicholson, J. Phillips, Ion diffusion modified by tortuosity and volume fraction in the extracellular microenvironment of the rat cerebellum, The Journal of Physiology, 321 (1981) 225-257.

[231] C. Nicholson, E. Syková, Extracellular space structure revealed by diffusion analysis, Trends in neurosciences, 21 (1998) 207-215.

[232] L. Longsworth, Diffusion measurements, at 25, of aqueous solutions of amino acids, peptides and sugars, Journal of the American Chemical Society, 75 (1953) 57055709.

[233] H. Kager, W.J. Wadman, G.G. Somjen, Simulated seizures and spreading depression in a neuron model incorporating interstitial space and ion concentrations, Journal of neurophysiology, 84 (2000) 495-512.

[234] C. Ayata, Spreading depression and neurovascular coupling, Stroke, 44 (2013) S87-S89.

[235] C.K. Willie, Y.C. Tzeng, J.A. Fisher, P.N. Ainslie, Integrative regulation of human brain blood flow, The Journal of physiology, 592 (2014) 841-859.

[236] H.H. Lipowsky, Microvascular rheology and hemodynamics, Microcirculation, 12 (2005) 5-15.

[237] I.G. Gould, A.A. Linninger, Hematocrit distribution and tissue oxygenation in large microcirculatory networks, Microcirculation, 22 (2015) 1-18.

[238] A.R. Pries, T.W. Secomb, P. Gaehtgens, J. Gross, Blood flow in microvascular networks. Experiments and simulation, Circulation research, 67 (1990) 826-834.

[239] A. Pries, T.W. Secomb, P. Gaehtgens, Biophysical aspects of blood flow in the microvasculature, Cardiovascular research, 32 (1996) 654-667.

[240] A. Pries, T. Secomb, T. Gessner, M. Sperandio, J. Gross, P. Gaehtgens, Resistance to blood flow in microvessels in vivo, Circulation research, 75 (1994) 904-915.

[241] A. Pries, T. Secomb, P. Gaehtgens, The endothelial surface layer, Pflügers Archiv, 440 (2000) 653-666.

[242] A. Pries, D. Neuhaus, P. Gaehtgens, Blood viscosity in tube flow: dependence on diameter and hematocrit, American Journal of Physiology-Heart and Circulatory Physiology, 263 (1992) H1770-H1778. 
[243] M.F. Kiani, A.R. Pries, L.L. Hsu, I.H. Sarelius, G.R. Cokelet, Fluctuations in microvascular blood flow parameters caused by hemodynamic mechanisms, American Journal of Physiology-Heart and Circulatory Physiology, 266 (1994) H1822-H1828.

[244] R.T. Carr, M. Lacoin, Nonlinear dynamics of microvascular blood flow, Annals of biomedical engineering, 28 (2000) 641-652.

[245] R. Guibert, C. Fonta, F. Plouraboué, Cerebral blood flow modeling in primate cortex, Journal of Cerebral Blood Flow \& Metabolism, 30 (2010) 1860-1873.

[246] G.M. Fraser, D. Goldman, C.G. Ellis, Microvascular flow modeling using in vivo hemodynamic measurements in reconstructed 3D capillary networks, Microcirculation, 19 (2012) 510-520.

[247] B.C. Fry, J. Lee, N.P. Smith, T.W. Secomb, Estimation of blood flow rates in large microvascular networks, Microcirculation, 19 (2012) 530-538.

[248] A.R. Pries, T.W. Secomb, Microvascular blood viscosity in vivo and the endothelial surface layer, American Journal of Physiology-Heart and Circulatory Physiology, 289 (2005) H2657-H2664.

[249] R. Fåhraeus, The suspension stability of the blood, Physiological Reviews, 9 (1929) 241-274.

[250] R. Fahraeus, T. Lindqvist, The viscosity of the blood in narrow capillary tubes, American Journal of Physiology-Legacy Content, 96 (1931) 562-568.

[251] A. Pries, K. Ley, M. Claassen, P. Gaehtgens, Red cell distribution at microvascular bifurcations, Microvascular research, 38 (1989) 81-101.

[252] J. Davis, C. Pozrikidis, Numerical simulation of unsteady blood flow through capillary networks, Bulletin of mathematical biology, 73 (2011) 1857-1880.

[253] R. Courant, K. Friedrichs, H. Lewy, Über die partiellen Differenzengleichungen der mathematischen Physik, Mathematische annalen, 100 (1928) 32-74.

[254] F. Cassot, F. Lauwers, C. Fouard, S. Prohaska, V. LAUWERS-CANCES, A novel three-dimensional computer-assisted method for a quantitative study of microvascular networks of the human cerebral cortex, Microcirculation, 13 (2006) 1-18.

[255] N. Safaeian, T. David, A computational model of oxygen transport in the cerebrocapillary levels for normal and pathologic brain function, Journal of Cerebral Blood Flow \& Metabolism, 33 (2013) 1633-1641.

[256] N. Safaeian, M. Sellier, T. David, A computational model of hemodynamic parameters in cortical capillary networks, Journal of theoretical biology, 271 (2011) 145156.

[257] A.F. Smith, V. Doyeux, M. Berg, M. Peyrounette, M. Haft-Javaherian, A.-E. Larue, J.H. Slater, F. Lauwers, P. Blinder, P. Tsai, Brain capillary networks across species: a few simple organizational requirements are sufficient to reproduce both structure and function, Frontiers in Physiology, 10 (2019). 
[258] A. Kapela, A. Bezerianos, N.M. Tsoukias, A mathematical model of Ca2+ dynamics in rat mesenteric smooth muscle cell: agonist and NO stimulation, $\mathrm{J}$ Theor Biol, 253 (2008) 238-260.

[259] D. Parthimos, D.H. Edwards, T.M. Griffith, Minimal model of arterial chaos generated by coupled intracellular and membrane Ca2+ oscillators, Am J Physiol, 277 (1999) H1119-1144.

[260] J. Yang, J.W. Clark, Jr., R.M. Bryan, C. Robertson, The myogenic response in isolated rat cerebrovascular arteries: smooth muscle cell model, Med Eng Phys, 25 (2003) 691-709.

[261] B.E. Carlson, D.A. Beard, Mechanical control of cation channels in the myogenic response, Am J Physiol Heart Circ Physiol, 301 (2011) H331-343.

[262] M.A. Spassova, T. Hewavitharana, W. Xu, J. Soboloff, D.L. Gill, A common mechanism underlies stretch activation and receptor activation of TRPC6 channels, Proc Natl Acad Sci U S A, 103 (2006) 16586-16591.

[263] L. Gagnon, A.F. Smith, D.A. Boas, A. Devor, T.W. Secomb, S. Sakadzic, Modeling of Cerebral Oxygen Transport Based on In vivo Microscopic Imaging of Microvascular Network Structure, Blood Flow, and Oxygenation, Front Comput Neurosci, 10 (2016) 82.

[264] N.M. Tsoukias, D. Goldman, A. Vadapalli, R.N. Pittman, A.S. Popel, A computational model of oxygen delivery by hemoglobin-based oxygen carriers in threedimensional microvascular networks, J Theor Biol, 248 (2007) 657-674.

[265] J.W. Ji, N.M. Tsoukias, D. Goldman, A.S. Popel, A computational model of oxygen transport in skeletal muscle for sprouting and splitting modes of angiogenesis, $\mathrm{J}$ Theor Biol, 241 (2006) 94-108.

[266] L. Gagnon, A.F. Smith, D.A. Boas, A. Devor, T.W. Secomb, S. Sakadžić, Modeling of cerebral oxygen transport based on in vivo microscopic imaging of microvascular network structure, blood flow, and oxygenation, Frontiers in computational neuroscience, 10 (2016) 82.

[267] Q. Fang, S. Sakadžić, L. Ruvinskaya, A. Devor, A.M. Dale, D.A. Boas, Oxygen advection and diffusion in a three dimensional vascular anatomical network, Optics express, 16 (2008) 17530.

[268] R. Hsu, T.W. Secomb, A Green's function method for analysis of oxygen delivery to tissue by microvascular networks, Math Biosci, 96 (1989) 61-78.

[269] T.W. Secomb, R. Hsu, E.Y. Park, M.W. Dewhirst, Green's function methods for analysis of oxygen delivery to tissue by microvascular networks, Ann Biomed Eng, 32 (2004) 1519-1529.

[270] T.W. Secomb, A Green's function method for simulation of time-dependent solute transport and reaction in realistic microvascular geometries, Math Med Biol, 33 (2016) 475-494. 
[271] L. Fenno, O. Yizhar, K. Deisseroth, The development and application of optogenetics, Annual review of neuroscience, 34 (2011). 
VITA

\title{
ARASH MOSHKFOROUSH
}

\author{
Born, Tehran, Iran
}

2003-2008

B.Sc., Materials Science and Engineering

Sharif University of Technology

Tehran, Iran

2008-2011 M.S., Materials Science and Engineering, Biomaterials

Sharif University of Technology

Tehran, Iran

2013 -2019 Ph.D, Biomedical Engineering

Florida International University

Miami, Florida

\section{PUBLICATIONS AND PRESENTATIONS}

Moshkforoush, A., Valdes-Hernandez, P.A., Rivera-Espada, D.E., Mori, Y. and Riera, J., 2018. "waveCSD: A method for estimating transmembrane currents originated from propagating neuronal activity in the neocortex: Application to study cortical spreading depression." Journal of neuroscience methods, 307, pp.106-124.

Moshkforoush A, Ashenaagr B, Mirza A, Longden T, Dabertrand F, Harraz OF, Nelson M, Tsoukias N., "Computational modeling of capillary-mediated neurovascular coupling: The dual role of Kir channels as sensors of neuronal activity and amplifiers of electrical signals" (Manuscript ready to be submitted to PNAS).

Moshkforoush A, Balachandar L, Moncion C, Santana J, Riera J. 2019. "Unraveling ChR2-driven stochastic Ca2+ dynamics in astrocytes-A call for new interventional paradigms." BioRxiv. 549469.

Moshkforoush A, Ashenagar B., Laffitte C., Tsoukias NM., Alevriadou R., "Modeling the role of endoplasmic reticulum-mitochondria microdomains in endothelial calcium dynamics" (Manuscript under preparation to be submitted to Nature Scientific Reports).

Koide M., Moshkforoush A., Tsoukias N.M., Hill-Eubanks D.C., Wellman G.C., Nelson M.T. and Dabertrand F., 2018. "The yin and yang of KV channels in cerebral small vessel pathologies." Microcirculation, 25(1), p.e12436. 
Ho I.L., Moshkforoush A., Hong K., Meininger G.A., Hill M.A., Tsoukias N.M. and Kuo W., 2016." Inherent rhythm of smooth muscle cells in rat mesenteric arterioles: An eigensystem formulation". Physical Review E, 93(4), p.042415.

Williams A., Nasim S., Salinas M., Moshkforoush A., Tsoukias N. and Ramaswamy S., 2017. "A "sweet-spot" for fluid-induced oscillations in the conditioning of stem cellbased engineered heart valve tissues." Journal of biomechanics, 65, pp.40-48.

Valdes-Hernandez PA, Bernal B, Moshkforoush A, Dunoyer C, Khoo HM, BoschBayard J, von Ellenrieder N, Gotman J, Riera JJ. "Identification of negative BOLD responses using windkessel models.” bioRxiv. 2018 Jan 1:392290.

Marzban M, Shahbazi A, Tondar M, Soleimani M, Bakhshayesh M, Moshkforoush A, Sadati M, Alizadeh Zendehrood S, Joghataei MT. "Effect of Mozart music on hippocampal content of BDNF in postnatal rats. Basic and Clinical Neuroscience." 2011 Apr 15;2(3):21-6.

Bakhtiary M, Marzban M, Mehdizadeh M, Joghataei MT, Khoei S, Mahabadi VP, Laribi B, Tondar M, Moshkforoush A. Comparison of transplantation of bone marrow stromal cells (BMSC) and stem cell mobilization by granulocyte colony stimulating factor after traumatic brain injury in rat. Iranian biomedical journal. 2010 Oct;14(4):142.

Gozalian A, Behnamghader A, Daliri M, Moshkforoush A. Synthesis and thermal behavior of Mg-doped calcium phosphate nanopowders via the sol gel method. Scientia Iranica. 2011 Dec 1;18(6):1614-22.

Moshkforoush A, Longden T, Dabertrand F, Harraz OF, Nelson M, Tsoukias N. "Kir mediates Regenerative and Directional Conduction of Hyperpolarization in Brain Capillaries: Importance for Neurovascular Coupling.” The FASEB Journal. 2018 Apr;32(1_supplement):712-12.

Moshkforoush A, Longden T, Dabertrand F, Nelson M, Tsoukias N. A Mathematical Model of Cerebral Blood Flow Control: Role of Kir Channels. The FASEB Journal. 2017 Apr;31(1_supplement):684-20.

Moshkforoush A, Tsoukias N. "Inward Rectifying Potassium (Kir) Channels and Spreading Vasodilation in the Cerebral Vasculature", 2016 BMES Annual Meeting, Oct 2016, Minneapolis, Minnesota, USA.

Moshkforoush A, Kapela A, Tsoukias N. "Multi-scale Modeling of Collecting Lymphatic Vessels: From Ion Channel Activity to Lymph Transport." The FASEB Journal. 2016 Apr;30(1_supplement):726-4.

Ashenagar B, Moshkforoush A, Mirza A, Tsoukias N. "Integrative Modeling of Functional Hyperemia: From Ion Channel Activity to Functional Imaging." The FASEB Journal. 2019 Apr;33(1_supplement):684-3. 